$\mathrm{DOE} / \mathrm{BC} / 14600-19$

Distribution Category UC-122

\title{
TRANSIENT FOAM FLOW IN POROUS MEDIA WITH CAT SCANIJER
}

\section{Topical Report}

By

Dianbin Liu

W. E. Brigham
$\mathrm{DOE} / \mathrm{BC} / 14600--19$

DE.92 001022

March 1992

Work Performed Under Cooperative Agreement No. FG22-90BC14600

Prepared for

U.S. Department of Energy Assistant Secretary for Fossil Energy

Thomas B. Reid, Project Manager

Bartlesville Project Office

P. O. Box 1398

Bartlesville, OK 74005

Prepared by

Stanford University

Petroleum Research Institute

Stanford, CA 94305-4042 


\section{Contents}

1 Introduction 1

2 Literature Review 3

2.1 Rheology And Flow Mechanism of Foam . . . . . . . . . . . . . 3

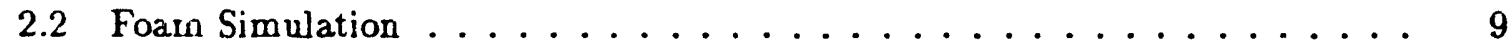

2.3 CAT Scanner Use in Porous Media . . . . . . . . . . . . . . . 10

3 Experimental Equipment and Procedures 11

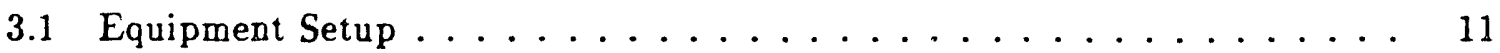

3.2 Experimental Procedures ....................... 15 
4.1 Fractional Flow Curves With No Mixing . . . . . . . . . . 25

4.2 The Effect of Mixing . . . . . . . . . . . . . . 32

4.3 Summary of the Theory ..................... . . 42

5 Results for Gas Displacements $\quad 46$

5.1 Displacements at Various Surfactant Concentrations . . . . . . . . 46

5.2 Surfactant Adsorption . . . . . . . . . . . . . . . 61

5.3 Matching of Gas Displacement Results . . . . . . . . . . . . 67

5.3.1 Mobility of Gas at Residual Liquid Saturation . . . . . . . . 67

5.3.2 Relative Permeabilities of Gas and Surfactant-Laden Water . . . . 68

5.3.3 Interpolating Equations for Gas Displacements . . . . . . . . 73

5.4 Matching of Pressure Data . . . . . . . . . . . . 89

6 Results of Foam Displacements $\quad 96$

6.1 Summary ố Foam Flow Experiments and Observations $\ldots \ldots \ldots$

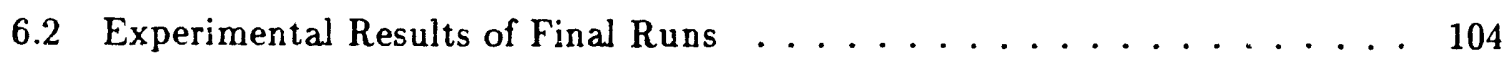

6.3 Theoretical Matching of Experimental Data . . . . . . . . . . 109

6.3.1 The Effect of Surfactant Adsorption on Foan Flow . . . . . . . 109

6.3.2 The Effect of Mixing on Foam Flow ............ 113 
6.3.3 Final Matching of Saturation Histories . . . . . . . . . . . 118

6.3.4 Matching of Recovery . . . . . . . . . . . . . 126

6.3.5 Matching of Pressure Drops . . . . . . . . . . . . 126

6.4 Some Implications of the Results . . . . . . . . . . . . . . . . 130

7 Conclusions

8 Recommendations for Future Work

$\theta$ Nornenclature

Bibliography

A Titration Method

B Data Acquisition

C Computer Program of Foam Simulation

D Computer Program of Mixing Effect

E Mixing Effect on Foam Displacements

F Pressure Calculation for Foam Flow 


\section{List of Tables}

4.1 Error Analysis wi Brigham Type Curve (Large Range of $\alpha / C \sigma$ ) . . . . 40

4.2 Error Analysis of Brigham Type Curve ( Small Range of $\alpha / C \sigma$ ) . . . . 42

5.1 Experimental Runs of Gas Displacement . . . . . . . . . . . . 47

5.2 Matching Data of Gas Displacements . . . . . . . . . . . 67

6.1 Experimental Runs of Foam Displacement . . . . . . . . . . . . 97

$6.2 \Delta X$ and $X$ Values for $\operatorname{Run} 41 \ldots \ldots \ldots \ldots$

$6.3 \Delta x$ and $\bar{X}$ values for Run $42 \ldots \ldots \ldots \ldots \ldots \ldots$

E.1 Calculation of Mixing Multipliers for Run $41 \ldots \ldots \ldots$

E.2 Calculation of Mixing Multipliers for Run $42 \ldots \ldots \ldots$ 


\section{List of Figures}

3.1 Simplified Experimental System Diagram . . . . . . . . . . . . . 12

3.2 Experimental System Setup with CAT Scanner . . . . . . . . . . . . 13

3.3 Short Sandpack Dimensions . . . . . . . . . . . . . . . 16

3.4 Long Sandpack Dimensions . . . . . . . . . . . . . . 17

3.5 Sandpack Packing Technique . . . . . . . . . . . . . . . 19

3.6 Relationship Between CT Number and Density (After Vinegar,1987) _ . . 21

4.1 Fractional Flow Curve Construction Method . . . . . . . . . . 28

4.2 Calculated Fractional Flow Curves for 20 Shocks (Example) . . . . . . . 31

1.3 Shock Velocities for 20 Shocks (Example) . . . . . . . . . . . 33

4.4 Saturation Profiles for 20 Shocks (Example) . . . . . . . . . . . 34

4.5 Liquid Saturation Profile on an EPV Basis (Example) . . . . . . . . 35

4.6 Recovery Efficiency History (Example) f . . . . . . . . . . . 36

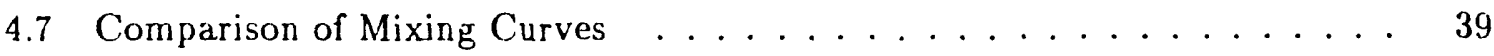


4.8 Semi-Empirical Mixing Equation Implementation . . . . . . . . . . 41

4.9 Type Curve Combining Mixing with Buckley-Leverett Theory . . . . . . 43

5.1 Saturation Histories for Run 21 (Gas Displacing Water) . . . . . . . 48

5.2 Pressure Responses for Run 21 (Gas Displacing Water) . . . . . . . . 49

5.3 Saturation Histories for Run 31 (Gas Displacing 0.001\% Surfactant-Laden

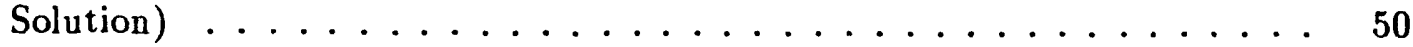

5.4 Pressure Responses for Run 31 (Gas Displacing 0.001\% Surfactant-Laden Solution $) \ldots \ldots \ldots \ldots \ldots \ldots$

5.5 Saturation Histories for Run 32 (Gas Displacing 0.01\% Surfactant-Laden

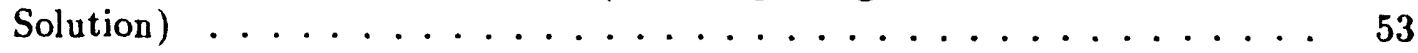

5.6 Pressure Responses for Run 32 (Gas Displacing 0.01\% Surfactant-Laden So-

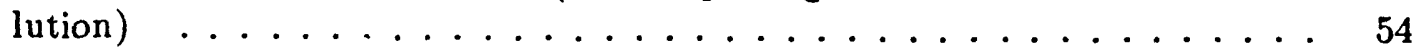

5.7 Saturation Histories for Run 33 (Gas Displacing 0.1\% Surfactant-Laden So-

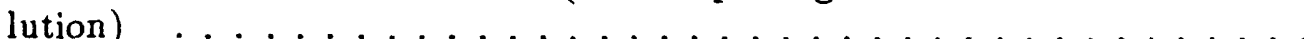

5.8 Pressure Responses for Run 33 (Gas Displacing 0.1\% Surfactant-Laden Sa-

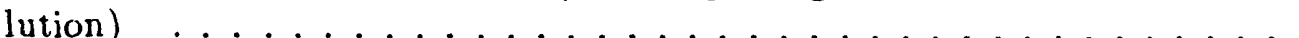

5.9 Saturation Histories for Run 35 (Gas Displacing 0.1\% Surfactant-Laden So-

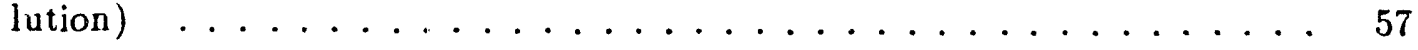

5.10 Pressure Responses for Run 35 (Gas Displacing 0.1\% Surfactant-Laden So-

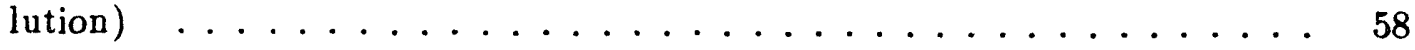

5.11 Saturation Histories for Run 34 (Gas Displacing 1\% Surfactant-Laden Solu-

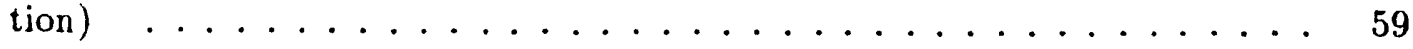

5.12 Pressure Responses for Run 34 (Gas Displacing 1\% Surfactant-Laden Solution) 
5.13 Surfactant Adsorption Measurements (at $0.01 \%$ ) . . . . . . . . 62

5.14 Surfactant Adsorption Measurements (at $0.1 \%$ ) . . . . . . . . 63

5.15 Surfactant Adsorption Measurements (at 1\%) . . . . . . . . . 64

5.16 Langmuir Adsorption Curve Match (Log-Log Graph) . . . . . . . . . 65

5.17 Langmuir Adsorption Match (Cartesian Graph) . . . . . . . . . . . 66

5.18 Saturation History Match for Run 21 (0.0\% Surfactant Concentration) . . 68

5.19 Saturation History Match for Run 31 (0.001\% Surfactant Concentration) 69

5.20 Saturation History Match for Run 32 (0.01\% Surfactant Concentration) . . 70

5.21 Saturation History Match for Run 35 (0.1\% Surfactänt Concentration) . . 71

5.22 Saturation History Match for Run 34 (1.0\% Surfactant Concentration) . . $\quad 72$

5.23 Calculated Gas Relative Permeabilities from Run 21 Data . . . . . . . . 74

5.24 Gas Relative Permeability of Run 31 from Experimental Data . . . . . . . 75

5.25 Relationship between Surfactant Concentration and Gas Saturation . . . 76

5.26 Relationship between Breakthrough Time and Surfactant Concentration • 78

5.27 Calculated Saturation Coefficient, $b$, as a Function of Surfactant Concentra-

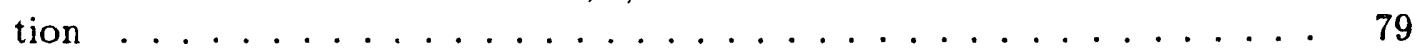

5.28 Apparent Viscosity as a Function of Surfactant Concentration . . . . . 80

5.29 Recovery Data Match for Run 21 (0.0\% Surfactant Concentration) . . . . 82

5.30 Recovery Data Match for Run 31 (0.001\% Surfactant Concentration) . . . 83 
5.31 Recovery Data Match for Run 32 (0.01\% Surfactant Concentration) . . . 84

5.32 Recovery Data Match for Run 35 (0.1\% Surfactant Concentration) . . . . 85

5.33 Recovery Data Match for Run 34 (1.0\% Surfactant Concentration) . . . . 86

5.34 The Effect of Interpolations on Fractional Flow Curves for Run $31 \quad \ldots$

5.35 Summary of the Fractional Flow Curves from Experiments $\ldots \ldots$. . . 88

5.36 Pressure Calculations for Run 21 (6.)\% Surfactant Concentration) . . . . 90

5.37 Pressure Calculations for Run 31 (0.001\% Surfactant Concentration) . . . 91

5.38 Pressure Calculations for Run 32 (0.01\% Surfactant Concentration) . . . . 9 92

5.39 Pressure Calculations for Run 35 (0.1\% Surfactant Concentration) . . . . 94

5.40 Pressure Calculations for Run 34 (1.0\% Surfactant Concentration) . . . . 95

6.1 Pressure Distribution versus PV Injected (Run 4$) \ldots \ldots . \ldots 9$

6.2 Pressure Distribution versus PV Injected (Run 9$) \ldots \ldots 9$

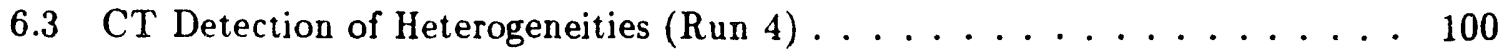

6.4 Relationship between CT Number and EPV Injected (Run 9, Location 1) • 102

6.5 CT Pictures from Run 10 (Location 1$) \ldots \ldots \ldots$

6.6 Saturation Histories for Run 41 ( $1 \%$ Surfactant Foam Displacing Water) . 105

6.7 Pressure Responses for Run 41 ( $1 \%$ Surfactant Foam Displacing Water) • 106

6.8 Saturation Histories for Run 42 ( 0.1\% Surfactant Foam Displacing Water) 107 
6.9 Pressure Responses for Run 42 ( 0.1\% Surfactant Foam Displacing Water) 108

6.10 Calculated Fractional Flow Curves for Foam of 1.0\% Surfactant Concentration ............................ 110

6.11 Calculated Saturation History for Foam of 1.0\% Surfactant Concentration 111

6.12 Calculated and Smoothed Saturation Histories for Foam of 1.0\% Surfactant Concentration ...................... 112

6.13 Effect of Surfactant Adsorption on Saturation Histories for Foam Flow of $1.0 \%$ Surfactant Concentration . . . . . . . . . . . . . . . 114

6.14 Saturation History Fits for Run 41 (1.0\% Surfactant Concentration) . . . 115

6.15 Saturation Profiles for Run 41 (1.0\% Surfactant Concentration) . . . . . . 116

6.16 Type Curve Matching Data for Run $41 \ldots \ldots \ldots \ldots$. . . . . . . . 117

6.17 Final Saturation History Match for Run 41 (Flow of 1.0\% Surfactant Foam) 119

6.18 Calculated Fractional Flow Curves for Foam of $0.1 \%$ Surfactant Concentration ......................... 120

6.19 Calculated Saturation History for Foam of $0.1 \%$ Surfactant Concentration 121

6.20 Calculated and Smoothed Saturation Histories for Foam of $0.1 \%$ Surfactant Concentration ..................... 122

6.21 Saturation History Fits for Run 42 (0.1\% Surfactant Concentration) . . . . 123

6.22 Saturation Profiles for Run 42 (0.1\% Surfactant Concentration) . . . . . . 124

6.23 Type Curve Matching Data for Run $42 \ldots \ldots \ldots \ldots$. . . . . . . . 125

6.24 Final Saturation History Match for Run 42 (Flow of $0.1 \%$ Surfactant Foam) 127 


\section{Acknowledgements}

The authors are grateful for the financial support from the Department of Petroleum Engineering of Stanford University, the SUPRI industrial associates and DOE through contracts No. DE-FG19-87BC14126 and DE-FG22-90BC14600. 


\section{Abstract}

Transient behavior is likely to dominate over most of the duration of a foam injection field project. Due to the lack of data, little is presently known about transient foam flow behavior. Foam flow does not follow established models such as the Buckley-Leverett theory, and no general predictive model has been derived. Therefore, both experimental data and a foam flow theory are needed.

In this work, foam was injected at a constant mass rate into one-dimensional sandpacks of 1 -in diameter and 24-in or 48-in length that had initially been saturated with distilled water. The system was placed in a CAT Scanner. Data, obtained at room temperature and low pressure at various times, include both the pressure and saturation distributions.

Pressure profiles showed that the pressure gradient is much greater behind the foam front than ahead of it. Moreover, the pressure gradients keep changing as the foam advances in the sandpack. This behavior differs from Buckley-Leverett theory. The CT scan results demonstrated gas channeling near the front, but eventually the foam blocks all these channels and sweeps the entire cross section after many pore volumes of injection.

Three series of experiments were run: (1) surfactant adsorption measurements, (2) gas displacements of surfactant-laden solutions and (3) foam displacements. The first two series of experiments were made to provide th^ necessary parameters required to match the foam displacements. To this end, it was necessary to smooth the saturation history data, using a Langmuir-type formula.

A theory was proposed based on the principles of the fractional flow curve construction method. This foam theory treats the foam as composed of infinitesimal slugs of gas of varying viscosities. The foam front has the lowest viscosity and foam at the injection end has the highest. 


\section{Chapter 1}

\section{Introduction}

There are various types of gas drives used for oil and gas recovery from petroleum reservoir Some are simple injections of gas such as methane; some are miscible gas injections suc.. as hydrocarbon and $\mathrm{CO}_{2}$ miscible flooding; and some are steam injections. These different injection methods all tend to override due to gravity, and channel and finger due to viscous instability and heterogeneity of the porous medium. Also gas has much lower viscosity than reservoir oils, which increases these effects. High permeability streaks within the reservoir may conduct most of the gasanus phase and leave much of the oil behind within the tight portions of the formation. Obviously anything that would help alleviate these kinds of problems would improve the ultimate recovery of gas and steam drives.

Through many laboratory and field experiments, foam has been used to compensate for this channeling effect (Marsden, 1986; Hirasaki, 1989). The for in is a combination of gas (either steam, $\mathrm{CO}_{2}$ or nitrogen) and a surfactant solution wh e usually the gas phase is discontinuous and the liquid is a continuous phase. It can be reated either in-situ in the reservoir or by simultaneous injection of the gas or steam and surfactant solution.

However, those experiments and nield projects to date have not rendered sufficient data to be generalized into a theory that could be used to predict future field applications. Most of the research found in the literature bas concentrated on various aspects of the steadystate flow of foam in porous media, whil: data indicate that the transient behavior is likely to dominate over most or all of the duration of a foam injection project (Liu et al., 1990). 
Foam simulation is still at an early stage. There are two main models available: population balance (Radke and Ransohoff, 1986; Ransohoff and Radke, 1988; Friedmann et al., 1991; Chang et al., 1990) and mechanistic models (Marfoe et al., 1987; Fisher et al., 1990; Falls et al., 1988; Huh et al., 1989), but they suffer from two weaknesses. They are very complex, and they do not have many experimental data to bacis them up.

Therefore, to understand the foam flow mechanism in porous media, transient foam flow experiments are needed. Moreover, a simple and effective flow model, based on the experiments, is also needed. 


\section{Chapter 2}

\section{Literature Review}

This literature survey was aimed mainly toward the rheology, flow mechanisms of foam and its use in enhanced oil recovery methods. There is an extensive review by Marsden (1986) of other aspects of foam flow in porous media. Since 1986 many more papers have been published in the literature or presented in conferences. It is worthwhile to mention that broad research efforts are being ui: iertaken worldwide on foam flow both microscopically and macroscopically. This shows the complexity of the problem with a promising future.

Foam simulation, although still at the early stage, is being extensively investigated and researched. So, many more papers on this subject are expected to be published in the near future. Since the CAT Scanner was used in this study, experiments involving this tool will also be briefly reviewed.

\subsection{Rheology And Flow Mechanism of Foam}

The survey will start with some background information. Gas drive is sometimes used in secondary recovery techniques, but gas drives are characterized by high gravity forces and high mobility ratios since gas typically has lower density and viscosity by an order of magnitude compared to reservoir oils. Thus gas drives are prone to gravity segregation, channeling and fingering, which cause early breakthrough with much of the oil left behind the displacing front. The same happens to some extent in miscible flooding.

The most widely used and most successful enhanced oil recovery methods are the various thermal recovery techniques. Among them, cyclic steam injection and steam drives are typically applied to heavy oil reservoirs with good results. Because of their higher ultimate 
recovery, steam drives are becoming the dominant thermal recovery technique. However, one of the biggest problems associated with steam injection is the early breakthrough of steam to the production well before all of the oil has been contacted, which is common for all gas drives.

The early breakthrough discussed above can also be affected by the heterogeneity of the reservoir. High permeability streaks within the reservoir may conduct most of the gaseous phase and leave most of the oil within the tighter portions of the formation. Obviously anything that would help alleviate one or more of these problems would improve the ultimate recovery from a steam drive. Hirasaki (1989) explained in detail the steam foam processes.

Some researchers considered the process of alternating water with steam (commonly called WASP) to both alleviate these problems and enhance the economics (Ault et al., 1985; Hong, 1987). While most of the papers reported success in recovering more oil over steam injection alone, a laboratory study by Shen (1989) claimed a difference of behavior when steam was injected first followed by hot water compared to hot water followed by steam. He found that while a hot waterflood followed by a steam injection recovered more oil from his homogeneous sandpack model, steam injection followed by hot water appeared to be ineffective. Shen did not repeat the injection for more than one cycle. His explanation for this was that the hot water following the steam injection would resaturate the depleted steam zone instead of going to the less permeable oil-filled region. This reasoning appears valid.

Sufi (1990), also using labcratory methods, conducted a series of WASP cycles (he called it SWAP instead) in to a horizontal well and concluded that the hot water injection displaces oil from a mobile zone and the steam pushes additional oil into the high permeability zone. $\mathrm{He}$ also predicted that this process should work equally well with a basal higher water saturated zone or a zone of fluid mobility created by heating .om a horizontal well. At the same time he observed that the oil production rate drops during each water injection period.

Hong (1989), after the successful test results of WASP on one field, was encouraged to continue the technique in the pilot area. Moreover, it was applied to another steamflood area in West Coalinger Field and considered for two other areas in the San Joaquin Valley. Hong further summarized the results as improved well productivity and reduced fuel consumption for steam generation. He mentioned that a simulation study was belpful in the design of a WASP project, and recommended that the WASP should be applied in other steam breakthrough areas.

Dornan (1990) discussed a case study of WASP in the Kern River Field, California. He disclosed that Texaco converted 275 steam displacement patterns to hot waterflood pilots 
in that field alone, however his conclusions may be a little disappointing. The evaluation of the process led to the belief that hot water was ineffective in transferring heat to the reservoir, hence the performance was poor. Simulation studies showed that recovery with injection shutdown while maintaining production w ould be superior to hot waterflooding.

For these reasons, one may realize that there have been both successes and failures in the use of WASP as an EOR method. It should be pointed out that foam might have been useful to help avoid the failures. Thus a promising method to improve these techniques is by the formation of an in-situ foam.

Foam has been used for a long time both in the laboratory and in the field. According to Marsden (1986), the first publication of any direct importance was a patent awarded to Bond and Holbrook (1958). There are many ways to generate foams, but Bond and Holbrook suggested that foam could be generated in an oil reservoir by consecutive injection of aqueous surfactant solution and gas. This appears to be a good method compared with the simultaneous injection of gas and suriactant solution. Bond and Holbrook considered foam as a displacing medium that would be less nobile than a gas and therefore have a more favorable mobility ratio relative to oil. It was claimed that sweep efficiency for both miscible and immiscible gas drives would thereby be increased.

Foam's high apparent viscosity could greatly improve the mobility ratio, and the blocking ability of foam can be useful in reducing the thieving effect of high permeability streaks on steam breakthro::gh. As reported by Lescure and Claridge (1986) and Falls et al. (1989), these beneficial characteristi ss could also be used to improve carbon dioxide flooding.

Not long after Bond and Holbrook (1958), Fried (1961) conducted a number of experiments using foam to displace brine and/or oil from porous media. For his experiments, Fried generated an aqueous foam external to the porous medium, injected a slug of foam into the medium, and then drove this slug along by continuous injection of air. Fried showed that the low mobility of the foam as well as its gas blocking ability contribute to a higher oil recovery and lower gas-oil ratio than with conventional displacement methods.

There are many variables and factors affecting the efficient use of foam. Most investigators concentrated on only one or two of them, including such variables as types of curfactants, temperature, pressure, formation, slug size, and presence of oil. The literature survey conducted by Marsden et al. (1977) indicated that the prime candidate for a blocking agent in steam drives was foam.

Chiang et al. (1980) investigated different surfactants and found that indeed, gravity override of gas could be sharply reduced. They also found that in-situ foaming generally 
increased with surfactant concentration until the critical micelle concentration (CMC) was reached. Additional surfactant beyond the CMC did not affect the foaming process. Wang et al (1982) studied the ability of several surfactants to generate foams at steam injection temperatures and pressures. They achieved promising results with several foamers despite surfactant degradation due to elevated temperatures.

Demiral and Okandan (1987), using a three-dimensional model, investigated the possibility of using in situ foam and steam in limestone formations. Under their test conditions, the optimum slug size was found to be $0.12 \mathrm{PV}$ at a surfactant concentration of $1.0 \%$. They showed that the foam generation improved the steam front movement and enhanced the heat flow to uninvaded zones, thereby increasing the oil recovery from the model by about $10 \%$. Robin (1985) also performed laboratory work on foaming additives to improve steam drive efficiency. Robin evaluated foam efficiencies from ambient conditions up to $300^{\circ} \mathrm{C}$ and $100 \mathrm{bar}$, and the effect of a residual oil phase on foam stability. He concluded that the foam stability of most surfactan is is considerably reduced when temperature increases, but a new discovery made was that this negative effect can be reduced by increasing the $\mathrm{pH}$, and by using different additives. Unfortunately, Robin did not give the real names of the surfactants he used.

Dilgren et al. (1982) described reduction of steam mobility by foam in terms of a permeability reduction factor defined as the ratio of the permeability of steam in the presence of foam to the permeability of steam with no foam. They found these factors ranging from 1.0 to 0.025 . For their purposes Dilgren et al. assumed that the mobility reduction was due to lowered permeability only, with no change in viscosity. This is contrary to most of the papers on this subject. Many people believed that foam has an apparent viscosity which is much higher than the viscosity of either its gas or liquid components.

Marsden and Khan (1966) measured bulk foam viscosities using a modified Fann VG Meter. They found the foam viscosity increased with increasing quality at a given shear rate. Some researchers concluded that foam was a non-Newtonian fluid because the apparent viscosity decreased with increased shear rate. Marsden and Khan found that increasing the surfactant concentration also increased the apparent viscosity slightly. These three basic properties of the viscosity of bulk foam have been confirmed in further studies by other researchers including Marsden and Khan (1966), Raza and Marsden (1967), Mitchell (1969), Minssieux (1974), Holbrook et al. (1981), Treinen (1985), and Lee et al. (1990). However, it was noted that it really makes little difference whether the experiments treated the foam flow behavior as an increase in viscosity or a decrease in permeability, for the ultimate equations always have these two parameters together. So the end result, to combine these viewpoints, is the reduction of mobility, which is a combination of viscosity and permeability. 
Foam flow has also been studjed on a laboratory scale in porous media (Hudgins and Chung, 1990; Bernard et al., 1965; Ettinger and Radke, 1989; Sanchez and Hazlett, 1989; Casteel and Djabbarah, 1988; Rossen, 1988). They studied different aspects of foams such as foam texture, apparent viscosity, microscopic behavior, and effect of adsorption. Through some experimental and theoretical studies, Lau and O'Brien (1988) showed that the transport of steam-foam surfactants through reservoir sands could be significantly retarded as a result of cation exchange between the surfactant solution and the formation clays.

Although many investigators did not agree with each other on the foam flow behavior, according to Marsden's (1986) review, the conclusion that foam acts like a non-Newtonian fluid is valid in most of the cases, including different media, different procedures and different surfactants. Moreover, foam most commonly behaved like either a pseudoplastic or a Bingham plastic fluid. This led Marsden and Khan (1966) to suggest the dual nature of foam in tubes. Of course, foam flow can be far more complex in reality.

There are as many disagreements on foam flow mechanisms as on foam rheological behavior. Beginning with Holm (1968), attempts have been made to establish the flow paths of gas and liquid when foam is injected into a porous medium. Holm (1968) concluded that foam does not flow as a body even when the liquid and gas were combined and injected as foam. There was no free foam flow; the gas moved through the system by progressively breaking and reforming bubbles through the length of the medium. The liquid was found to move via the interconnected film netrork of the bubbles. The respective flow rates were a function of the number and strength of the films. The stability and bubble making ability of liquid films (lamellae) has, therefore, become the crux of many recent studies.

The behavior of foam lamellae in smooth capillary tubes was the subject of a project undertaken by Hirasaki and Lawson (1983). They emphasized the importance of: (1) foam texture, or average bubble size in relation to the capillary tube size; (2) foan quality, or the gas volume divided by the total volume; and (3) the surface tension gradients created when surfactant is swept from the front of a bubble to its rear. A more recent work on the subject has dealt with the conditions under which foam bubbles are formed and/or destroyed using simplified models such as a toroidally constricted capillary tube described by Sanchez and Schechter (1986). They formulated an expression for the generation rate of foam bubbles based on geometric and fluid properties.

Radke arıd Ransohoff (1986) categorized the mechanisms of foam generation within glass bead packs. They concluded that snap-off was the primary mechanism responsible for the formation of a strong foam. They developed a simple model to predict the onset of snap-off.

The stability of foam lamellae as determined by capillary pressure was the subject of a study by Khatib et al. (1986). The destabilization of foam lamellae by oil droplets 
was observed by Nikolov et al. (1986) using sophisticated microscopic techniques. They demonstrated that the surfactant type and concentration directly influence the stability of the three phase emulsion structure.

Aside from all the references above, many observations have been proposed to describe the flow mechanisms. They can be summarized as the following seven types of patterns (Mahmood and Brigham, 1987): (1) Bubble flow, (2) Intermittent flow, (3) Plug flow, (4) Trapped-Gas flow, (5) Segregated flow, (6) Membrane flow, and (7) Tubular-Channel flow.

One can see that there are also many disagreements among the investigators concerning foam fiow mechanisms. Microscopic studies are essential and helpful in understanding the foam flow mechanisms. It can be seen that foam is a very complex fluid. Much work needs to be done, especially experimentally, to really understand the rheology and flow mechanisms, and hence to describe it mathematically.

Even before there was much basic understanding of foam behavior, field tests of the technique were underway (Holm and Garrison, 1988; Mohammadi and Tenzer, 1990; Yang et al., 1988). All of them had some success on oil recovery. Significantly higher oil production rates were reported by Doscher and Kruskraa (1983) after addition of a foaming agent into a steamdrive pattern. Dilgren et al. (1982) noticed increased bottom-hole injection pressures and higher oil production rates when surfactant and nitrogen were added to a steam flood operation in the Kern River Field. These are but two demonstrations of the usefulness of this promising technique.

Castanier (1989) did a literature survey and discussed the topic of steam with additives citing, the field projects of the eighties. He compared sixteen field tests of steam with additives (foam) which had a broad spectrum of reservoirs, oils, depths and pressures. In addition, various surfactants were screened and used. Overall, as Castanier concluded, the use of additives with steam can provide significant benefits over the use of steam alone. The addition of surfactants to the steam (to form foam) has proven to be both technically and economically successful as loug as some simple criteria are met.

Friedmann et al. (1991) did a field trial of steam foam injection in the Midway-Sunset Field. It was found that the buttom-hole pressure increased from $100 \mathrm{psig}$ to $300 \mathrm{psig}$, which indicated foam generation. Friedmann et al. also observed that a better steam distribution provided the conditions for the generation of foam. Improvements in both vertical and areal sweep efficiency were reported. A large amount of incremental oil was produced.

Most laboratory studies have dealt with the steady state behavior under foam injection. One approach not yet thoroughly reported is the investigation of the transient displacement 
and pressure behavior of a foam injection process. Since truily steady state conditions can not be achieved before a considerable amount of foam has been introduced into a porous medium, the transient forces are likely to dominate over most of a field project.

Liu et al. (1990) described some experiments to study the transient behavior of foam in one-dimensional porous media with the help of a CAT Scanner. It was found that the transient foam behavior differs from Buckley-Leverett displacement theory and gas channeling (or fingering) occurs at the foam front, suggesting a non piston-like displacement.

\subsection{Foam Simulation}

For a number of years there has been considerable interest in numerical simulation of foam flow (Marfoe et al., 1987; Radke and Ransohoff, 1986; Fisher et al., 1990). However, as mentioned above, the mechanics of foam displacement are not well understood. Thus the numerical models proposed can only fit a small fraction of the experiments (Friedmann et al., 1991; Ransohoff and Radke, 1988; Falls et al., 1988; Huh et al., 1989; Chang et al., 1990).

Marfoe et al. (1967), in simulating foam flow in porous media, modeled the foam behavior as gas-liquid dispersions. Then they adopted the black-oil model equations for oil-water-gas systems, modifying the gas by considering surfactant concentration and gas interstitia! velocity. However, they could not evaluate the soundness of their model due to lack of experimental data.

Two main models have been suggested in the literature, population balance model (Radke and Ransohoff, 1986; Ransohoff and Radke, 1988; Friedmann et al., 1991; Chang et al., 1990) and mechanistic model (Marfoe et al., 1987; Fisher et al., 1990; Falls et al., 1988; Huh et al., 1989). Both these models are very complex. Each has many variables and factors. In addition, since these models did not consider the effect of mixing, they all fall in to the category of Buckley-Leverett theory to some extent. That is, they assume that the distance of movement of all saturations is directly proportional to the volume injected. Finally there were not many experimental data available to test the models. 


\subsection{CAT Scanner Use in Porous Media}

It may be worth mentioning that the Computed Tonography is also known by many other names (Brooker, 1986). Among them, the most popular ones are computed axial tomography (CAT), computer aided tomography (CAT), computed transverse axial tomography (CTAT), computed transmission tomography (CTT), reconstruction tomography (RT), selective computer assisted tomography (SCAT), and transmission axial tomography (TAT). Throughout this work, CAT Scanner is used to refer to the equipment itself and CT is used to refer to the use of the CAT Scanner as in CT number and CT scanning.

Ever since its introduction int $n$ the petroleum industry in the early eighties, the CAT Scanner has been used widely (L. . et al.,1990; Vinegar and Wellington, 1987; Vinegar, 1986; Peters and Hardham, 1990; Hove et al., 1987; Hove et al., 1990; Hunt et al., 1988; Wang et al., 1984; Tomutsa, 1991; Wellington and Vinegar, 1985). The uses of the CAT Scanner have varied greatly, ranging from studies of geology, to core analyses, and flow experiments. At Stanford University alone, the CAT Scanner is presently being used as a tool for the investigation of foam mechanisms, pattern steam foam flow, and end effect due to capillarity. To date, at least a dozen oil companies and half a dozen universities around the world have used CAT Scanners in their research. It may be worth mentioning that very little of the CAT Scanner-assisted research has been devoted to the study of foam flow mechanisms. 


\section{Chapter 3}

\section{Experimental Equipment and Procedures}

The flow equipment used in this study was originally designed and used by Treinen (1985) for the study of the apparent viscosity of foam during steady state flow in porous media. Many modificatiors to that design have been made for the current experiments to investigate the transient behavior of foam flow. One major modification is the addition of the CAT (Computer Aided Tomography) Scanner into the system. Another change is the upgrading of the computerized data acquisition system by replacing the old Apple Plus computer with a new IBM-compatible 386-based PC. Also, a longer saridpack was designed and used for some of the experimental runs. Since so many modifications were made, the equipment will be described as a whole without mentioning the differences between the original design and the modifications.

\subsection{Equipment Setup}

A simplified system diagram is shown in Figure 3.1. The addition of the CAT Scanner is illustrated in Figure 3.2.

A Matheson mass flow meter (Model 8240) was used to inject nitrogen at a constant rate. Liquid (water or surfactant solution) was delivered through an LDC/Milton Roy constaMetric III G metering pump. Foam was created by the simultaneous injection of nitrogen and surfactant solution through a tube containing sand of sizes 60 through 100 mesh. The sandpack was made of transparent acrylic tubing. It was filled with Ottawa sand, to be further described later. 


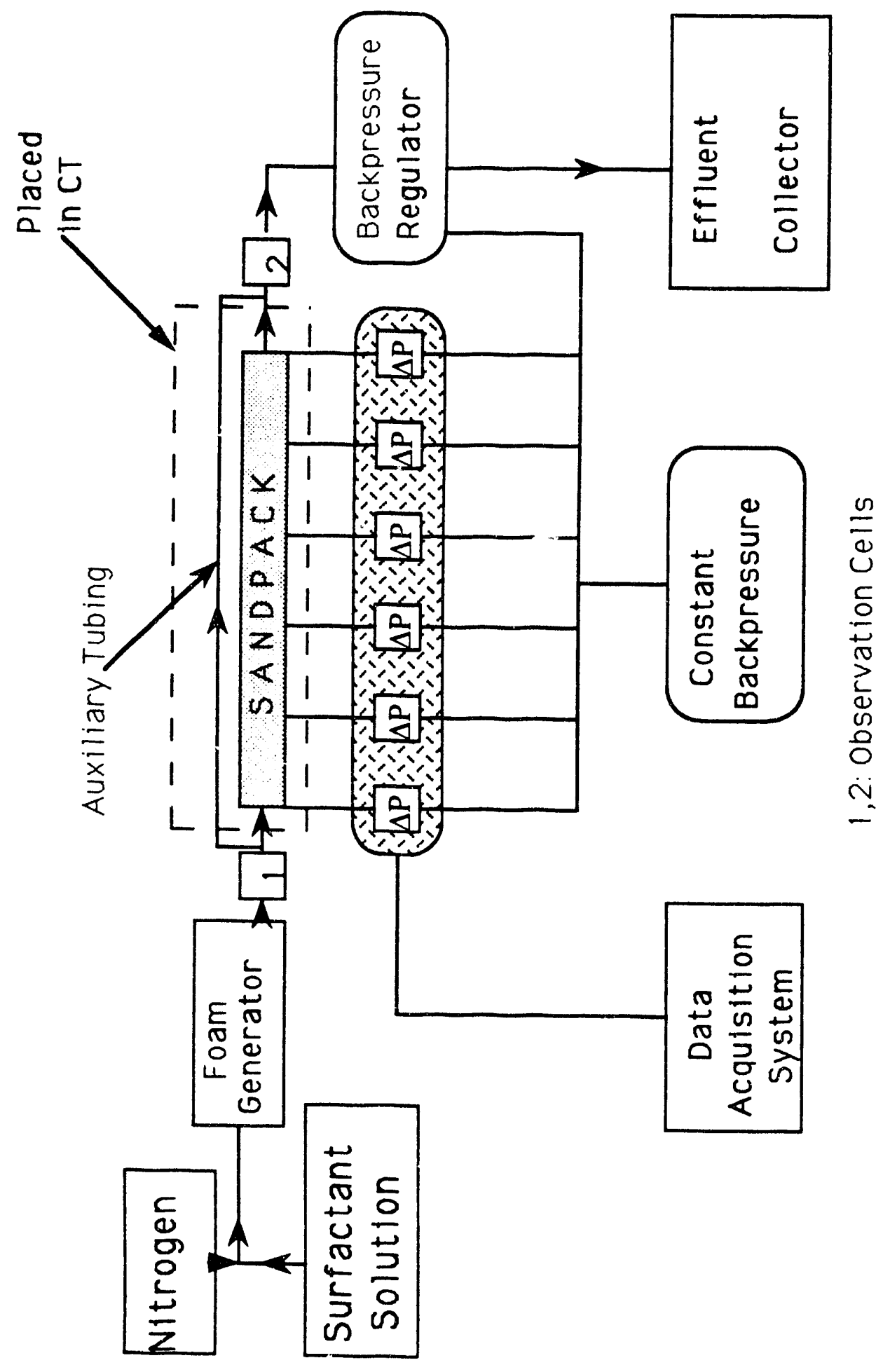

Figure 3.1: Simplified Experimental System Diagram 


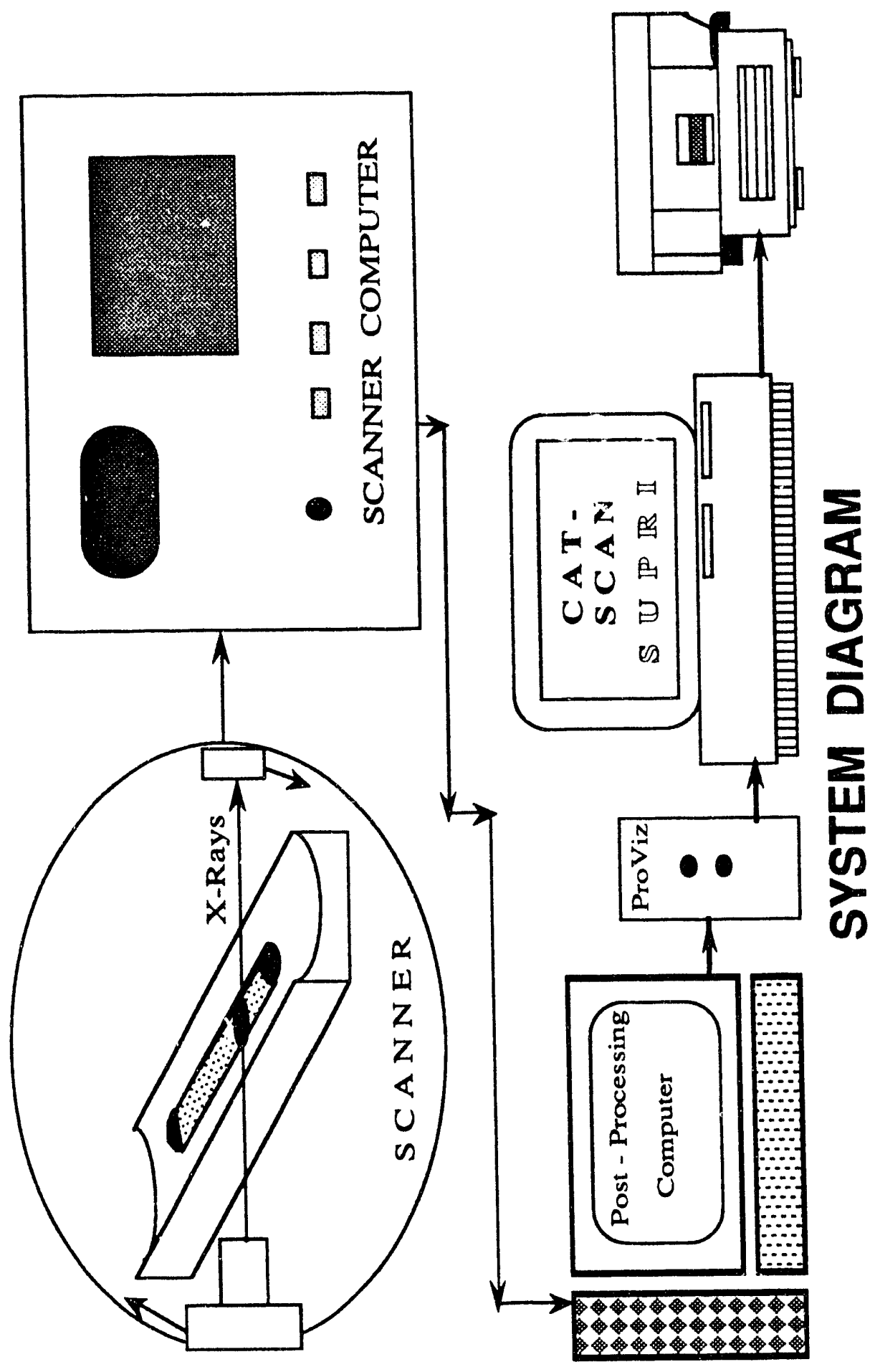

Figure 3.2: Experimental System Setup with CAT Scanner 13 
There were transparent observation cells at the inlet and outlet of the sandpack, which enabled one to observe the foam flow outside the sandpack. The entire sandpack was placed in the $\mathrm{CT}$ gantry. The system also had an auxiliary tubing bypassing the sandpack. By closing or opening the auxiliary inlet and outlet valves, the injected fluids could be flowed either through the sandpack or directly into the effluent collector. Pressures at various points (to be described later) along the sandpack were recorded with pressure transducers and demodulators (Celesco CD 10D and CD 25A). The electric signals from the pressure demodulators were then transferred to an HP data acquisition/control unit (Model 3497A) which was connected to an IBM-compatible computer.

A constant back pressure was maintained for all the pressure transducers and at the outlet. The pressures were taken against the back pressure and measured through the data acquisition system. The data acquisition program on the computer is shown in Appendix B. Due to the fact that there was only one back pressure regulator controlling both the pressure transducers and the outlet, a pressure change in the regulator could cause changes in all the devices it is connected to. Hence care must be taken to watch the pressure variations in the regulator so as to minimize the changes. Fortunately, the major concern was the prissure difference or drop between the transaucers and the back pressure instead of the at:iolute pressures of the individual transducers. That is, the pressure variations in the back pressure regulator would not affect the results as significantly as they appear to.

The effluent liquid was collected at the outlet of the sandpack with a Buchler Fractomette Alpha 200 fractometer. Then it was analyzed by weighing and/or titration to obtain the fractional flow and surfactant concentration.

The CT system used for this study was an EMI 5005, second-generation (translaterotate) type scanner. For the basic principles of CAT scanners, the interested reader is referred to Morgan (1983) and Brooker (1986). A mechanical table was added to the CAT Scanner to move the sandpack back and forth with precision. Padding materials (rubber foam in this case) were wrapped around the sandpack at the desired scanning locations.

The sandpack was then mounted inside the CT gantry on to a mechanical table to be scanned. To eliminate the CT artifact due to non-symmetrical effects, the sandpack was positioned precisely to the center of the CT gantry with the help of two laser beams which were mounted on the walls on each side of the CAT Scanner. To position the sandpack in the center of the CT gantry would also provide CT ir ages that could be used for 3-D image construction.

After each scan on one cross section, the data were transferred to the scanner computer and then to the post-processing computer where the C.T numbers were obtained. The image data were then transferred to a Macintosh II computer in which various soft ware systems 
(Image. Ultimage) were used for further analysis. The image could also be printed as a hard copy on a laser printer. All the image data were backed up on tapes.

The sand used in the sandpack was 100-200 mesh Ottawa sand which had been acid and base washed. Sand migration was prevented by 200 mesh stainless steel screens at the inlet and outlet, and by ir. line filters with 60 micron porous elements at the pressure tap fittings.

To minimize variation in foam quality due to expansion of gas, the system was initially run at a downstream pressure of 50 psig. This was achieved by using a backpressure regulator. Two sandpacks were used in the experiments. They were of the same inner diametcr ( 1 inch or $2.54 \mathrm{~cm}$ ) and were 24 inches $(60.96 \mathrm{~cm}$ ) and 48 inches $(121.92 \mathrm{~cm})$ long. To measure the absolute pressure along tha sandpack eight pressure taps were located at 2. 4, 6, 9, 12, 15, 18, and 21 in. from the inlet (Figure 3.3), for che short sandpack. The inlet and outlet, along with six of the sandpack iaps, were ccanected to jifferential pressure transducers. For the longer sandpack (Figure 3.4), there were six pressure taps evenly distributed 8 inches $(20.32 \mathrm{~cm})$ apart, learing 4 inches $(10.16 \mathrm{~cm})$ at the inlet and the outler.

\subsection{Experimental Procedures}

Three series of experiments were run. The first one was the disf acement of distilled water by nitrogen. The second series was the displacement of various roncentrations of surfactantladen solution by nitrogen. Finally the third series was the displacement of distilled water by foam made up of surfactant laden solution and nitrogen. In all these experiments, the only differences were the saturation of the in-situ liquid and the nature of the displacing fluid. All the other techniques were the same including pressure measurement $\therefore$ CT scanning and effluent collection.

In the first experiments, the sandpack was initiaily saturated with distilled water. Then nitrogen was injected at a constant rate to displace the water. In the second series of experiments, the sandpack was initially saturated with surfactant solvtiviz of predetermined concentration. Then nitrogen was introduced at a constant rat 2 . The third series of experiments were a little more complicated. Hence the following is devoted mainly to the discussion of these foam dispiacements.

Prior to each experimental run, the pressure transducers were calibrated and the transducer demodulators were adjusted to the appropriate range, 5 psig or $25 \mathrm{psig}(0.34 \mathrm{~atm}$ or 


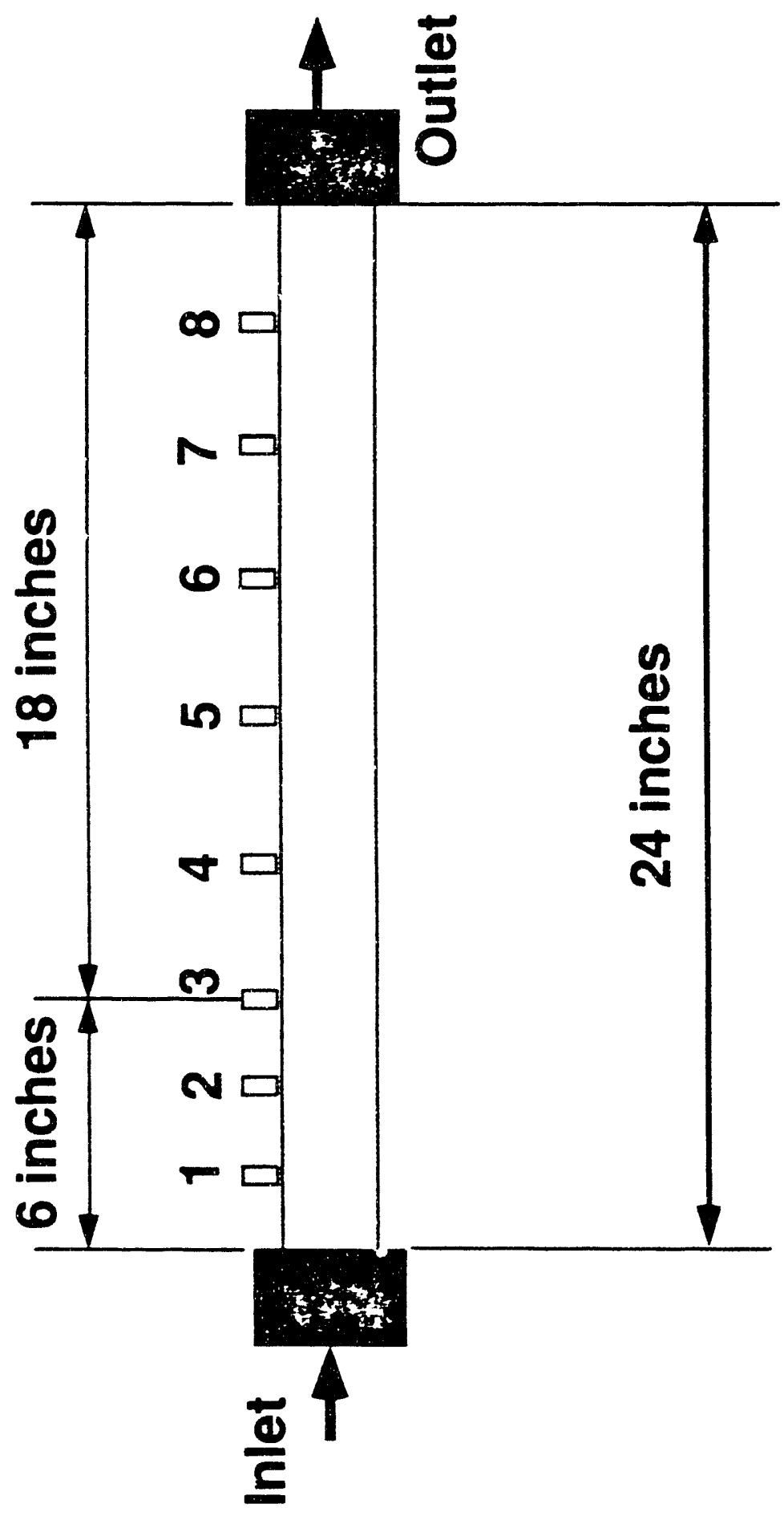

Figure 3.3: Short Sandpack Dimensions 


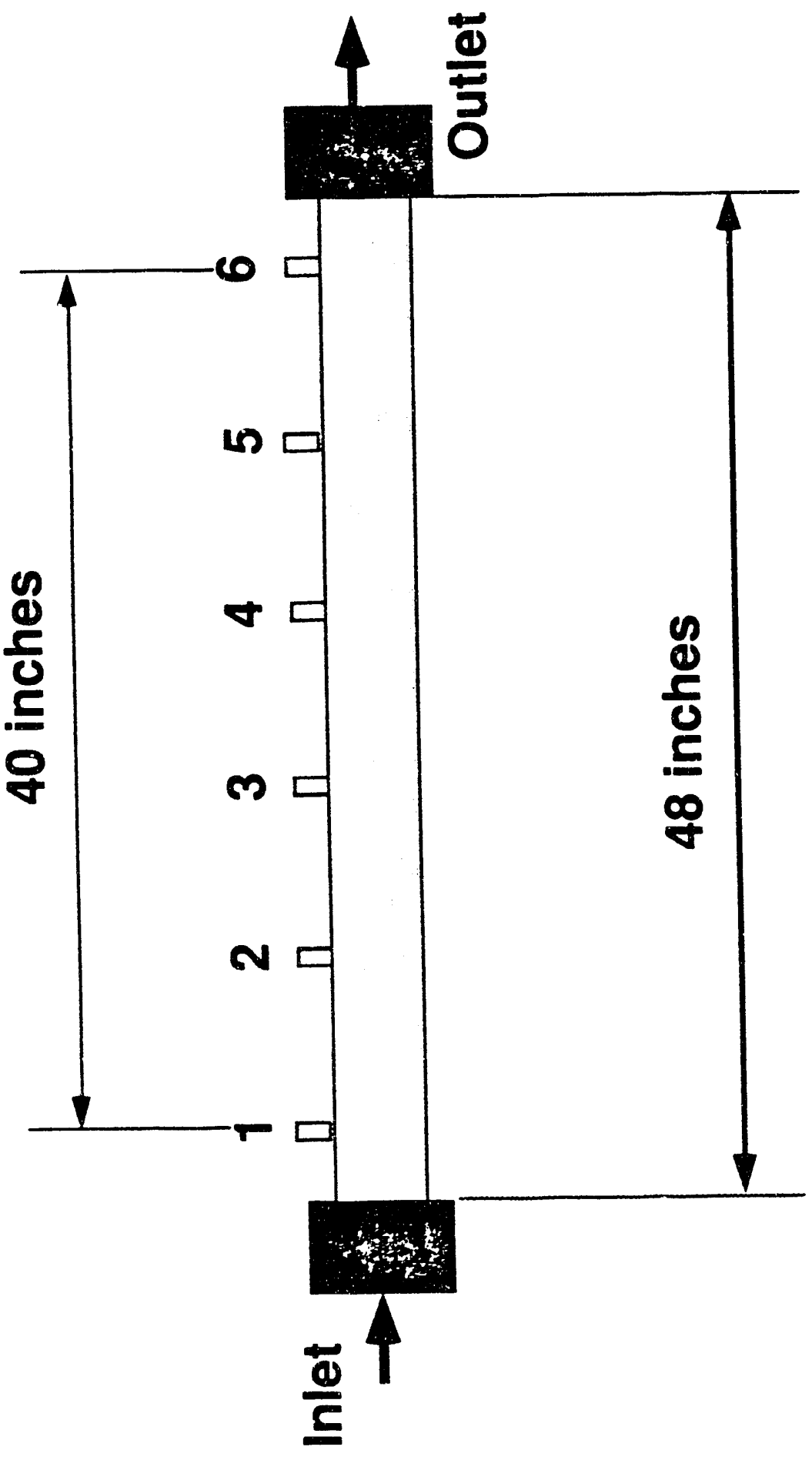

Figure 3.4: Long Sandpack Dimensions 
$1.70 \mathrm{~atm})$. Each run was begun with a new sandpack. This was to assure that the sand was not contaminated by surfactant from the previous run. Dry sand was packed into the tube held in an upright position, while vibration and an induced vacuum were used to induce settling of the sand (Figure 3.5). Then distilled water was introduced from the bottom of the sandpack to achieve the beneficial effect of wet packing. After the sandpack was fully saturated with water, $\mathrm{CO}_{2}$ was used to displace the water from the top down. During this entire process, vibration was continued. This process was repeated for a few times before the sand was packed homogeneously. To ensure consistency, each sandpack's porosity and permeability were measured before each run. The porosity was estimated by the weight (volume) of distilled water saturating the sandpack compared to that of the sand volume. The sandpack was then mounted on to the experimental system. More water was pumped in. The permeability of the sandpack was calculated from the pressure drop and the water flow rate. Verification of homogeneity in porosity was made by comparing the CT scanning results at various locations.

Foam of known quality and flow rate was generated by passing nitrogen and surfactant solution simultaneously through a foam generator. The foam was then injected into a sandpack $100 \%$ saturated with water. Foam flow rate, quality, surfactant concentration, and back pressure were first selected for each experiment.

The surfactant concentration was calculated from the weights of surfactant and the water used. It should be remembered that usually the surfactants are not $100 \%$ active. So the activity' of the surfactant was also a factor. The following equation was used:

$$
C=\frac{W_{s} \times A_{s}}{W_{w}+W_{s}}
$$

where $C$ is the surfactant concentration, $W_{s}$ is the weight of surfactant, $W_{w}$ is the weight of water, and $A_{s}$ the activity of the surfactant.

Foam quality could be calculated by a ratio of volumes.

$$
\text { Quality, } \Gamma=\frac{\text { Gas Volume }}{\text { Gas Volume + Liquid Volume }}
$$

The difficulty in obtaining a desired quality arises due to the compressible nature of gas. The foam quality and flow rate were therefore calculated at the midpoint of the sandpack using an anticipated pressure gradient. These expected pressure gradients were derived from Treinen's steady state data. It soon became clear, for the transient data, that the average pressure was not going to remain constant. Thus the quality would vary during a run. There being no better means, however, this method was continued. The problem 


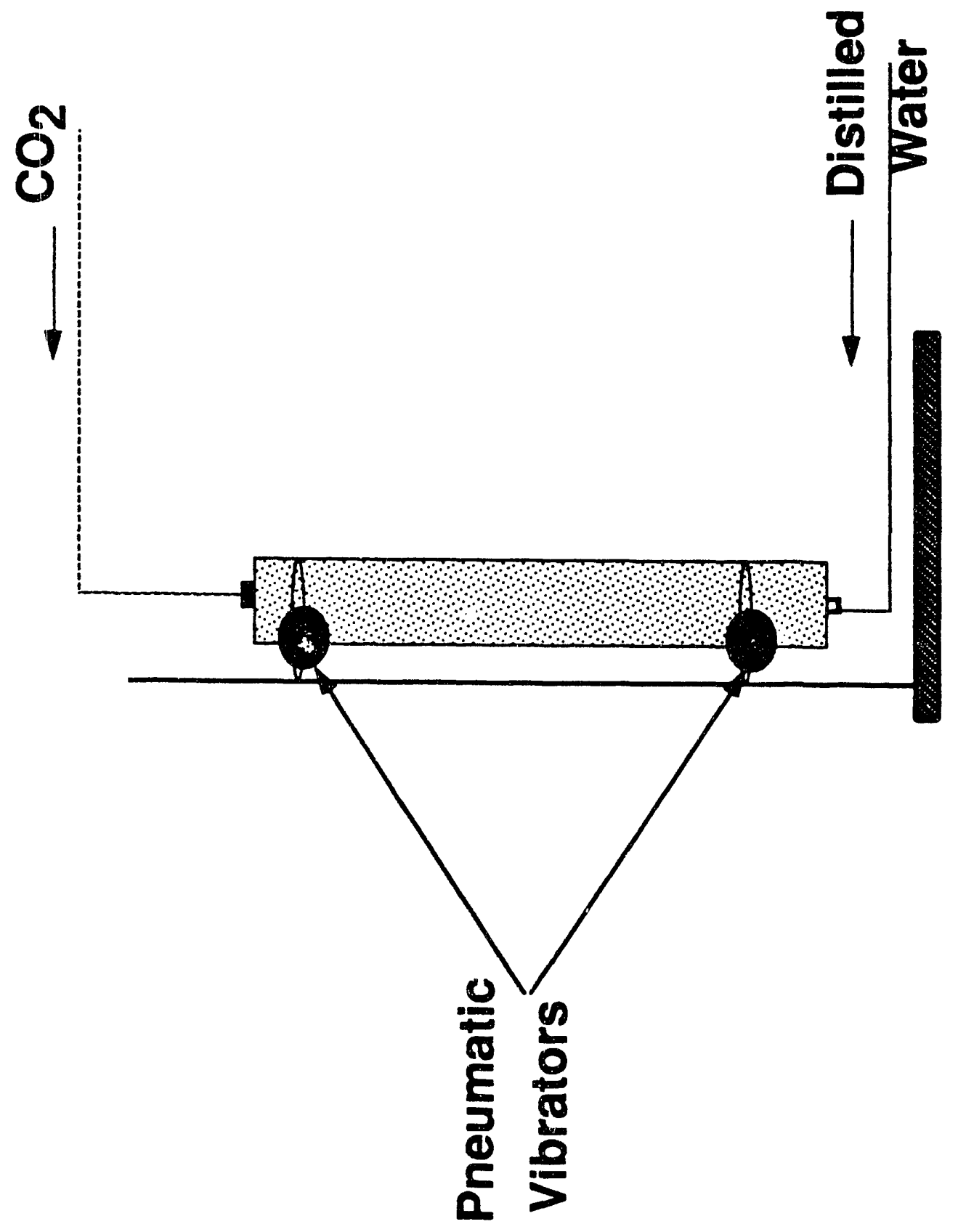

Figure 3.5: Sandpack Paching Technique 
could be remedied to some extent by the use of the effluent pressure to calculate quality. The back pressure also helped to reduce the variations in quality.

The gas flow rate at reservoir condition was then found:

$$
q_{g}=\frac{14.7\left(q_{g}\right)_{s c}}{14.7+\Delta p / 2+p_{b}}
$$

where $q_{g}$ is the gas flow rate in $c c / \mathrm{min},\left(q_{g}\right)_{s c}$ is the gas flow rate at standard conditions (cc/min),$\Delta p$ is the anticipated pressure drop over the sandpack during the experiment in psi, and $p_{b}$ is the back pressure in psig. The back pressure was set at 50 psig in all the experiments.

The liquid phase was assumed to be incompressible. The total foam flow rate is a simple addition:

$$
q_{\text {foam }}=q_{l}+q_{g}
$$

where $q_{\text {foam }}$ is the foam flow rate, and $q_{l}$ is the liquid flow rate.

The desired foam was generated and allowed to flow through the upstream observation cell ind out the auxiliary inlet, while the sandpack remained water saturated, sealed, and pressurized. When the foam flow was constant and stabilized through the auxiliary outlet, the auxiliary inlet was closed and the foam pressure was allowed to build up to the sandpack pressure. The foam was then introduced into the sandpack and pressure monitoring, observations, effluent collection and CT scanning begun.

The collected liquid samples were first weighed to find their volume and then titrated using a chloroform-hyamine procedure (Appendix A) to find the surfactant (sulfonate) concentration. By knowing the volumes of the samples, their surfactant concentrations, and the times over which they were taken, a material balance could be performed for the surfactant in order to determine in-situ concentration. The surfactants used in this study were AOS 1618 of ENORDET and Suntech IV.

The sandpack was placed under the CAT Scanner during the displacement process. The CAT Scanner measures the attenuation of $X$-rays in a material. CT number is used as a measure of how much the X-ray's are absorbed by the materials (Morgan, 1983). In general, CT numbers are linearly proportional to the density of the materials. Figure 3.6 shows the relationship betvieen the $\mathrm{CT}$ number and the bulk density of some materials (Vinegar and Wellington, 1987). The CT numbers in this study range approximately from 300 to 600 . 


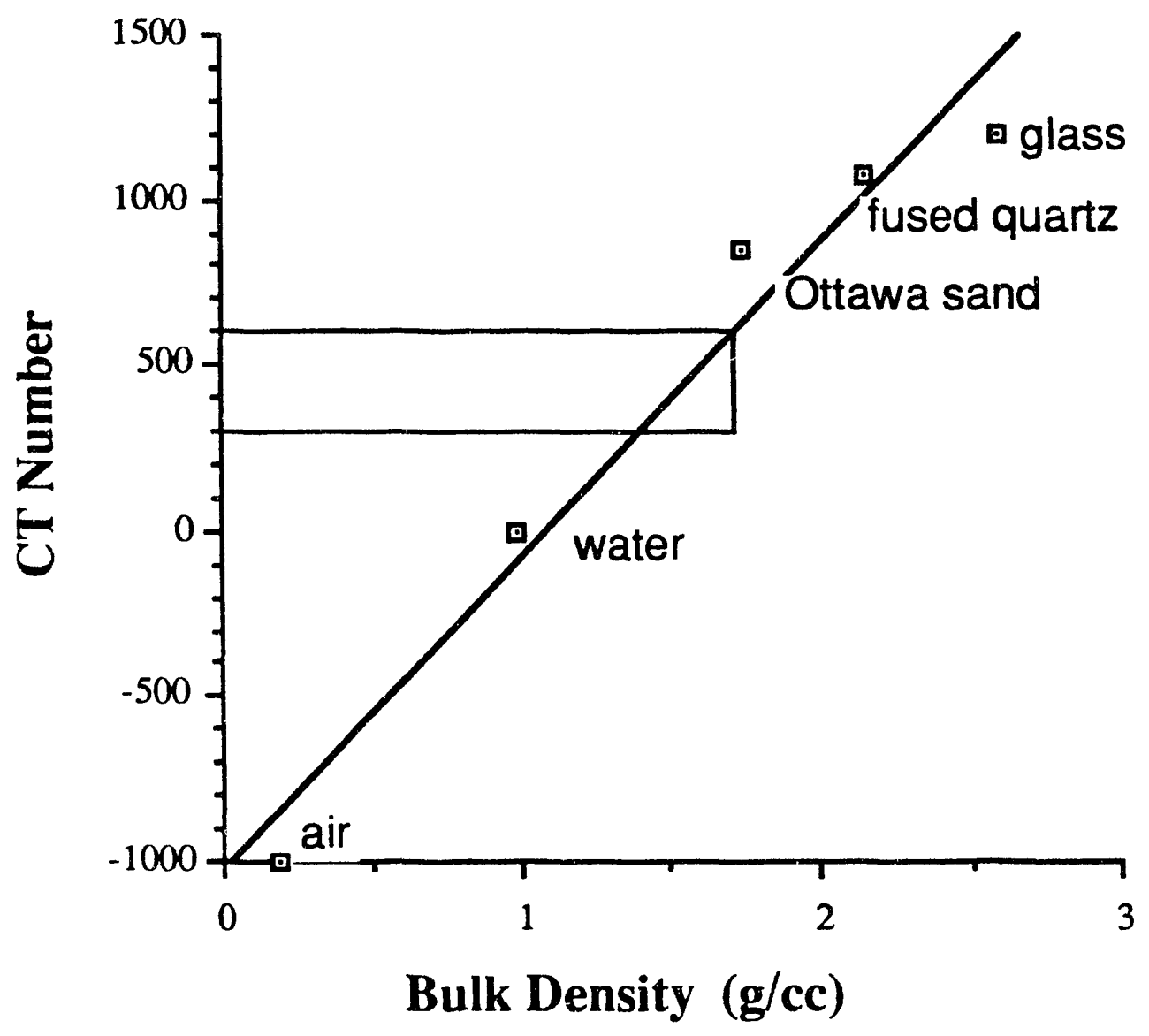

Figure 3.6: Relationship Between CT Number and Density (After Vinegar,1987) 
The linear attenuation coefficient of a cross section, for two-phase flow, can be represented by:

$$
\mu=\mu_{w} S_{w}+\mu_{g} S_{g}
$$

where $\mu$ is the linear attenuation coefficient of a cross section containing both liquid and gas, $\mu_{w}$ is for a cross section containing only water and $\mu_{g}$ for gas, while $S_{w}$ and $S_{g}$ are water and gas saturations.

For each scan, a CT number was obtained from the CAT Scanner computer. In this system, the CT number was calculated from:

$$
N_{C T}=\frac{\mu-\mu_{w}}{\mu_{w}} \times \text { Const }
$$

where $N_{C T}$ is the CT number. The constant in the equation depends on the calibration of the CAT Scanner using different phantoms (air or water). In this work, it was determined to be about 1000 . It should be noted that this constant has no effect on the calculated saturations, as shown in the next equation.

The liquid saturation at any cross-section of the sandpack was then estimated from Equations 3.5 and 3.6. Also considering that the saturations of gas and water must add up to one (1) for two phase flow, the following equation is obtained for liquid saturation:

$$
S_{l}=\frac{N_{C T}-\left(N_{C T}\right)_{g}}{\left(N_{C T}\right)_{l}-\left(N_{C T}^{\prime}\right)_{g}}=\frac{\mu-\mu_{g}}{\mu_{w}-\mu_{g}}
$$

where $S_{l}$ is the liquid saturation. $\left(N_{C T}\right)_{l}$ and $\left(N_{C T}\right)_{g}$ are the CT numbers for the sandpack saturated with water and gas only, and $N_{C T}$ is the CT number for a system containing both phases.

Since each experiment begins with a new sandpack, several cross sections of each sandpack were scanned while fully saturated with distilled water and analyzed as a basis for future comparisons in the same run. Once the experiment started and foam was flowing in to the sand-pack, all the predetermined cross-sections were scanned in sequence by moving the sandpack using the mechanical table to obtain CT number-time relationships, which in turn relate to the saturation through Equation 3.7. At the end of each experiment, the foam had completely displaced the distilled water from the sand-pack. A CT scan at this time gave yet another basis for comparison with other scans for intermediate stages. 
A CT number is obtained from the computer on an average basis. It is a measure of all the voxels of interest on that cross section. A voxel is simply a three-dimensional volume pixel. Since a CT number is a statistical average, every CT number for a given section is associated with a standard deviation measured by taking into account all the voxels in the section. The greater the standard deviation, the more heterogeneous the cross section; hence the standard deviation is an indication of the amount of gas channeling on that cross section. For this preliminary work no detailed quantitative analysis was made of every voxel of a cross section. However, a good estimate of the saturation distribution can be made directly from the CT images.

All the data collected through the pressure data acquisition system, the effluent samples, and the C.T scanning were analyzed as described in detail in Chapters 5 and 6. 


\section{Chapter 4}

\section{Theoretical and Numerical Procedures}

In this chapter, a new, simple mathematical model will be proposed for foam flow in porous media. The central idea is to treat foam as an infinite number of gas slugs with differing viscosities displacing the in-situ fluid. Due to adsorption of surfactant on to the sand surface, and due to the changing apparent viscosity of the gas-foam mixtures, the gas ahead of the foam front breaks out of the foam and never coalesces back into foam. Hence the gas at the front has the viscosity of pure gas. Towards the inlet, where all the adsorption sites have been filled by previous surfactant-laden injected fluid, the foam is more stable. The gas phase at the injection end, therefore, assumes the apparent viscosity of the injected foam, which is usually orders of magnitude greater than that of pure gas. The theory behind this is the fractional flow curve construction method which has also been used for polymer flooding (Lake, 1989).

Foam flow is accompanied by both adsorption and mixing. Mixing contributes to the flow behavior in a way which is different from Buckley-Leverett displacement theory. The effect of mixing was found to be important enough that it had to be dealt with separately.

The following assumptions will be made to simplify the problem:

A1. The porous medium is uniform and isotropic with respect to all relevant properties:

A2. All fluids are incompressible, including the foam phase. (This assumption is approximately valid as long as the pressure drop across the porous medium is small, as was the case in tinis work).

A3. The effect of pressure on phase equilibrium and relative permeabilities is negligible.

A4. The temperature is constant with distance and time. 
A5. Partial molar volumes of components are constant, with no change in total volume upon transfer of components from one phase to another.

A6. The phases are in local equilibrium everywhere.

A7. There is no stagnant space left in the porous media during the process.

A8. The solid phase is neither deformable nor moveable.

A9. The injected foam is considered to act as a single phase (in fact, since the foam quality is $90 \%$, the foam phase is often referred to as "gas" phase in this work).

A10. Only surfactant can be adsorbed onto the sand surface, while the other components can not.

A11. The flow is one-dimensional.

The theory is described in two parts - the method without mixing and the effect of mixing.

\subsection{Fractional Flow Curves With No Mixing}

The fractional flow curve construction method is also referred to as Generalized Welge Construction. Helfferich (1981) (1982) described the theory of multicomponent, multiphase displacement in porous media as well as the construction method for two-phase flow in porous media in systems with limited miscibility.

The fractional flow curve construction method starts with a set of fractional flow curves, each one of which represents a gas "slug" of a certain viscosity displacing the in-situ fluid. In our case the in-situ fluid is water.

To obtain the fractional flow curves, the relative permeabilities must be determined first. To illustrate this procedure, the following relative permeability equations are used:

$$
\begin{aligned}
& k_{r w}=k_{r w}^{0}\left(\frac{S_{w}-S_{w c}}{1-S_{w c}-S_{g r}}\right)^{n_{w}} \\
& k_{r g}=k_{r g}^{0}\left(\frac{S_{g}-S_{g r}}{1-S_{w c}-S_{g r}}\right)^{n_{g}}
\end{aligned}
$$


where

$k_{\tau w}=$ water relative permeability

$k_{r g}=$ gas relative permeability

$k_{r g}^{0}=$ endpoint relative permeability for water at residual gas saturation

$k_{r w}^{0}=$ endpoint relative permeability for gas at irreducible water saturation

$S_{w}=$ water saturation

$S_{g}=$ gas saturation

$S_{g r}=$ residual gas saturation

$S_{w c}=$ irreducible water saturation

$n_{w}=$ relative permeability index for water

$n_{g}=$ relative permeability index for gas

Using Equations 4.1 and 4.2, the fractional flow of gas for horizontal flow is calculated as follows:

$$
f_{g}=\frac{M^{0}\left(S_{g}^{*}\right)^{n_{g}}}{M^{0}\left(S_{g}^{*}\right)^{n_{0}}+\left(1-\left(S_{g}^{*}\right)\right)^{n_{w}}}
$$

where

$$
\begin{aligned}
f_{g} & =\text { fractional flow for gas } \\
S_{g}^{*} & =\text { reduced gas saturation, }\left(S_{g}-S_{g r}\right) /\left(1-S_{w c}-S_{g r}\right) \\
M^{0} & =\text { gas-water endpoint mobility ratio, }\left(k_{r g}^{0} \mu_{w}\right) /\left(k_{r w}^{0} \mu_{g}\right) \\
\mu_{w} & =\text { waler viscosity, cp } \\
\mu_{g} & =\text { "gas" viscosity, cp, in this case the "gas" is actually foam. }
\end{aligned}
$$


The actual fractional flow curves can be obtained directly from experiments, as will be described later in Chapter 5. However, the manner of calculating the displacement results will be the same as outlined below.

The method of making the appropriate calculations is illustrated in Figure 4.1. As shown, there are a series of gas fractional flow curves (four in this figure), which illustrate the effects of changing the gas (or foam) viscosity. The fractional flow curve for the lowest gas viscosity is at the left. The higher the gas viscosity, the further to the right is its fractional flow curve.

Since the surfactant adsorption is significant at the foam (gas) front, it can not be neglected. The frontal advance loss due to adsorption can take a variety of forms. At this stage, a Langmuir-type isotherm (Langmuir, 1915) has been chosen as the basis. It will be modified if experimental data require it. It should be noted that although the frontal advance loss is dimensionless, it has to be compatible with the saturation, i.e. it is based on the pore volume. Combining the fractional flow curve construction method and the adsorption loss, the first gas shock velocity is calculated as:

$$
v_{1}=\frac{f_{g_{1}}-f_{g_{0}}}{S_{g_{1}}+D_{1}}=f_{g_{1}}^{\prime}
$$

where the Langmuir adsorption is:

$$
D_{1}=\frac{a}{1+b C_{1}}
$$

where

$$
\begin{aligned}
v_{1} & =\text { velocity of the first gas shock front } \\
f_{g_{1}} & =\text { gas fractional flow at first shock } \\
f_{g_{0}} & =\text { gas fractional flow at initial condition } \\
f_{g_{1}}^{\prime} & =\text { tangent of first fractional flow curve } \\
S_{g_{1}} & =\text { gas saturation at first shock } \\
D_{1} & =\text { frontal advance loss of surfactant at first shock due to adsorption }
\end{aligned}
$$




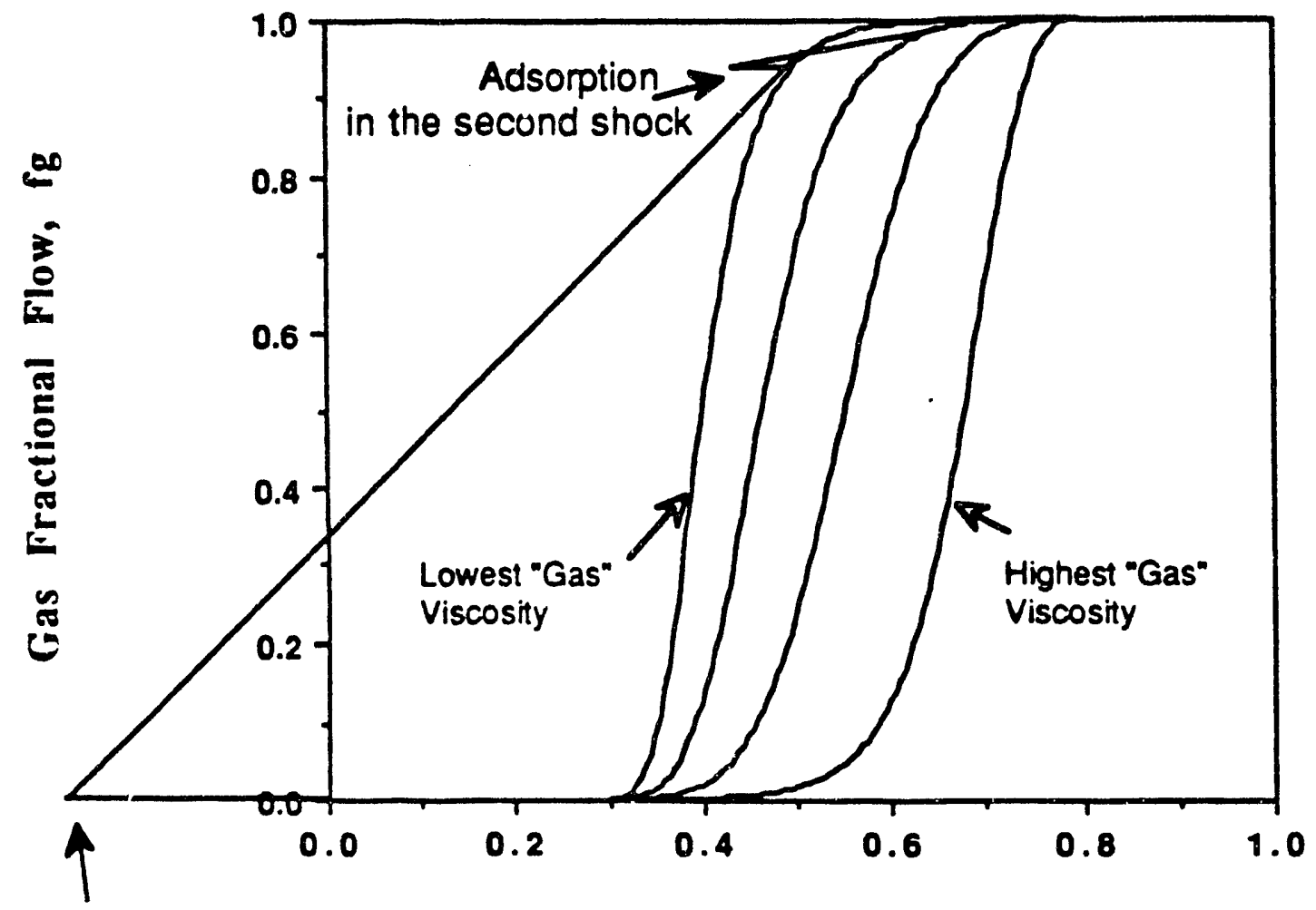

Adsorption

in the first shock

Gas Saturation, Sg

Figure 4.1: Fractional Flow Curve Construction Method 28 
$a, b=$ Langmuir constants

$C_{1}=$ Surfactant concentration at first shock

For the second shock, the velocity is:

$$
v_{2}=\frac{f_{g_{2}}-f_{g_{1}}}{\left(S_{g_{2}}+D_{2}\right)-\left(S_{g_{1}}+D_{1}\right)}=f_{g_{2}}^{\prime}
$$

and the Langmuir adsorption is:

$$
D_{2}=\frac{a}{1+b C_{2}}
$$

where

$$
\begin{aligned}
& v_{2}=\text { second gas shock velocity } \\
& f_{32}=\text { gas fractional flow at second shock } \\
& f_{g_{2}}^{\prime}=\text { tangent of second fractional flow curve } \\
& S_{g_{2}}=\text { gas saturation at second shock } \\
& D_{2}=\text { frontai advance loss of surfactant at second shock due to adsorption } \\
& C_{2}=\text { surfactant concentration at second shock }
\end{aligned}
$$

In general, the velocity of the $n$th gas shock $(n \geq 2)$ can be expressed in a manner similar to Equations 4.6 and 4.7 as follows:

$$
v_{n}=\frac{f_{g_{n}}-f_{g_{n-1}}}{\left(S_{g_{n}}+D_{n}\right)\left(S_{g_{n-1}}+D_{n-1}\right)}=f_{g_{n}}^{\prime}
$$


where the Langmuir adsorption terms are:

$$
\begin{aligned}
D_{n} & =\frac{a}{1+b C_{n}} \\
D_{n-1} & =\frac{a}{1+b C_{n-1}}
\end{aligned}
$$

where

$$
\begin{aligned}
v_{n} & =n \text {th gas shock velocity } \\
f_{g_{n}} & =\text { gas fractional flow at } n \text {th shock } \\
f_{g_{n}}^{\prime} & =\text { tangent of } n \text {th fractional flow curve } \\
S_{g_{n}} & =\text { gas saturation at } n \text {th shock } \\
D_{n} & =\text { frontal advance Langmuir adsorption loss at } n \text {th shock } \\
C_{n} & =\text { surfactant concentration at } n \text {th shock } \\
f_{g_{n-1}} & =\text { gas fractional flow at }(\mathrm{n}-1) \text { th shock } \\
S_{g_{n-1}} & =\text { gas saturation at (n-1)th shock } \\
D_{n-1} & =\text { frontal advance Langmuir adsorption loss at }(\mathrm{n}-1) \text { th shock } \\
C_{n-1} & =\text { surfactant concentration at }(\mathrm{n}-1) \text { th shock }
\end{aligned}
$$

According to the above equations for shock velocities, the first shock velocity is equal to the slope of the tangent to the first fractional flow curve (the lowest gas velocity) starting from $f_{g}=0$ and $S_{g}=-D_{1}$, as illustrated in Figure 4.1. The second shock velocity is the slope of the tangent to the second fractional flow curve (the next higher gas viscosity) starting from $f_{g}$ at the first shock and at a gas saturation value which is $S_{g}$ of the first shock minus $\left(D_{2}-D_{1}\right)$. The rest of the shocks are treated the same way.

A computer program (listed in Appendix C) was written to implement these concepts. The following curves were calculated using that program. From this program, for example, the fractional flow curves are shown in Figure 4.2 for a series of gas slugs displacing water with gas viscosities ranging from $0.02 \mathrm{cp}$ to $20 \mathrm{cp}$. The viscosities were interpolated so that 
$a, b=$ Langmuir constants

$C_{1}=$ Surfactant concentration at first shock

For the second shock, the velocity is:

$$
v_{2}=\frac{f_{g_{2}}-f_{g_{1}}}{\left(S_{g_{2}}+D_{2}\right)-\left(S_{g_{1}}+D_{1}\right)}=f_{g_{2}}^{\prime}
$$

and the Langmuir adsorption is:

$$
D_{2}=\frac{a}{1+b C_{2}}
$$

where

$v_{2}=$ second gas shock velocity

$f_{g_{2}}=$ gas fractional flow at second shock

$f_{g_{2}}^{\prime}=$ tangent of second fractional flow curve

$S_{32}=$ gas saturation at second shock

$D_{2}=$ frontal advance loss of surfactant at second shock due to adsorption

$C_{2}=$ surfactant concentration at second shock

In general, the velocity of the $n$th gas shock $(n \geq 2)$ can be expressed in a manner similar to Equations 4.6 and 4.7 as follows:

$$
v_{n}=\frac{f_{g_{n}}-f_{g_{n-1}}}{\left(S_{g_{n}}+D_{n}\right)-\left(S_{g_{n-1}}+D_{n-1}\right)}=f_{g_{n}}^{\prime}
$$


where the Langmuir adsorption terms are:

$$
\begin{aligned}
D_{n} & =\frac{a}{1+b C_{n}} \\
D_{n-1} & =\frac{a}{1+b C_{n-1}}
\end{aligned}
$$

where

$$
\begin{aligned}
v_{n} & =n \text {th gas shock velocity } \\
f_{g_{n}} & =\text { gas fractional flow at } n \text {th shock } \\
f_{g_{n}}^{\prime} & =\text { tangent of } n \text {th fractional flow curve } \\
S_{g_{n}} & =\text { gas saturation at } n \text {th shock } \\
D_{n} & =\text { frontal advance Langmuir adsorption loss at } n \text {th shock } \\
C_{n} & =\text { surfactant concentration at } n \text {th shock } \\
f_{g_{n-1}} & =\text { gas fractional flow at (n-1)th shock } \\
S_{g_{n-1}} & =\text { gas saturation at (n-1)th shock } \\
D_{n-1} & =\text { frontal advance Langmuir adsorption loss at (n-1)th shock } \\
C_{n-1} & =\text { surfactant concentration at }(\mathrm{n}-1) \text { th shock }
\end{aligned}
$$

According to the above equations for shock velocities, the first shock velocity is equal to the slope of the tangent to the first fractional flow curve (the lowest gas velocity) starting from $f_{g}=0$ and $S_{g}=-D_{1}$, as illustrated in Figure 4.1. The second shock velocity is the slope of the tangent to the second fractional flow curve (the next higher gas viscosity) starting from $f$ at the first shock and at a gas saturation value which is $S_{g}$ of the first shock minus $\left(\nu_{2}-D_{1}\right)$. The rest of the shocks are treated the same way.

A computer program (listed in Appendix C) was written to implement these concepts. The following curves were calculated using that program. From this program. for example, the fractional flow curves are shown in Figure 4.2 for a series of gas slugs displacing water with gas viscosities ranging from $0.02 \mathrm{cp}$ to $20 \mathrm{cp}$. The viscosities were interpolated so that 


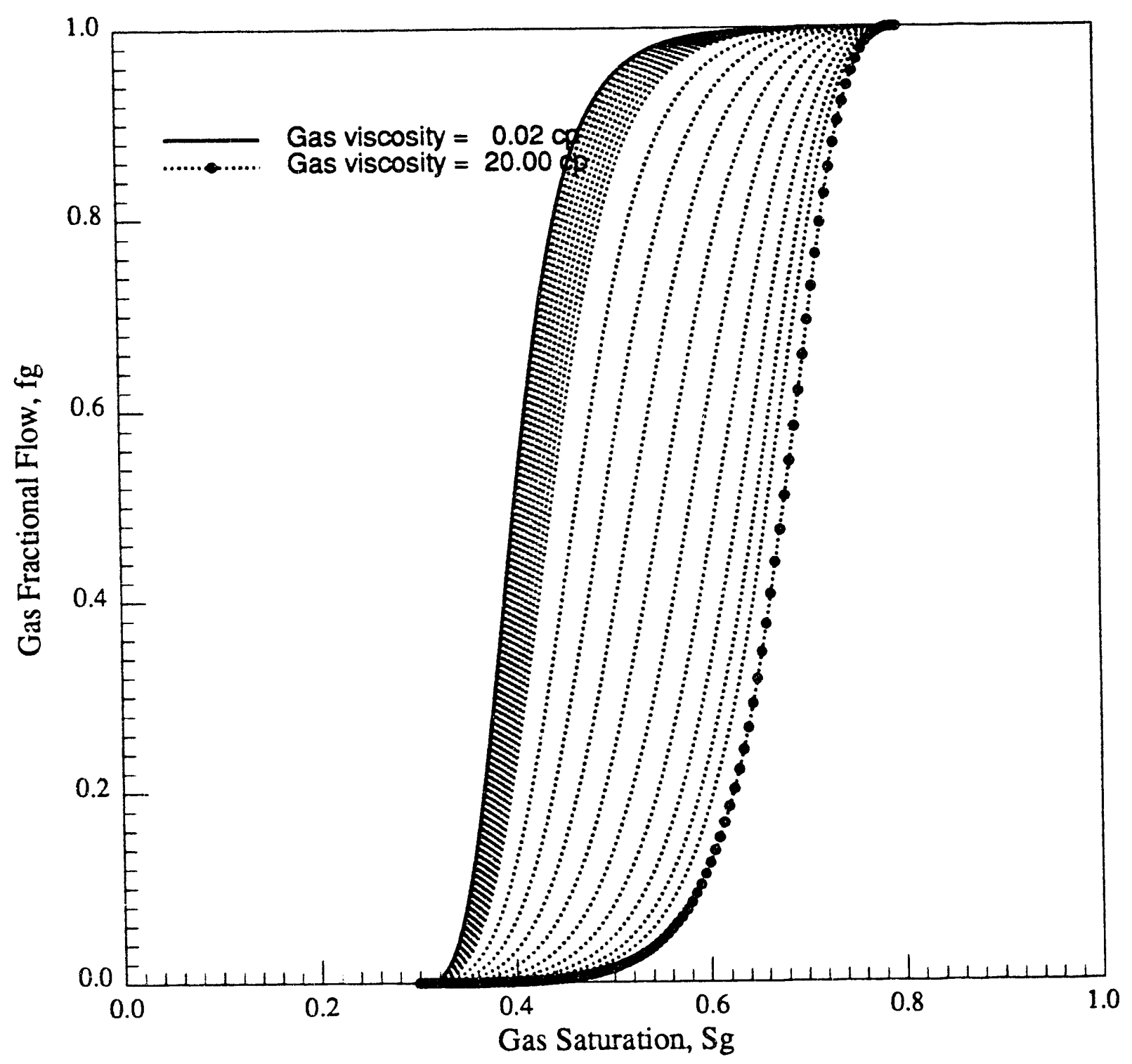

Figure 4.2: Calculated Fractional Flow Curves for 20 Shocks (Example) 31 
the later saturation history would be sinooth. It should be noted that the interpolation method does not affect the final results since theoretically, there are an infinite number of interpolations. Here for numerical convenience, only 20 different viscosities are considered. The first tangential point on the first curve (for $0.02 \mathrm{cp}$ ) gives rise to the gas flow fraction and the gas saturation for the first shock. The rest of the shocks were obtained as described in Equations 4.6 to 4.10 above. The material balance due to adsorption was taken into account to modify the shock velocities. Figure 4.3 shows the velocities for each shock represented by the dimensionless quantities: dimensionless distance, $X_{D}$ and dimensionless time, $t_{D}$.

For a given time, the saturation profile can readily be obtained from the velocities of each shock, as shown in Figure 4.4 at $0.5 \mathrm{PV}$ injected. For comparison, the saturation profiles are also calculated for the cases of pure gas displacements at viscosities $0.02 \mathrm{cp}$ and $20 \mathrm{cp}$. As can be seen, the saturation profile for foam flow falls between the pure gas displacements, as expected. Notice that the displacement curve follows more closely the lower gas viscosity curve.

Saturation changes can also be shown as a graph of the saturation as a function of the equivalent pore volumes injected at a given location. Figure 4.5 shows the liquid saturationEPV relationship at the outlet $\left(X_{D}=1\right)$ for the fractional flow curves above. Also the recovery efficiency can be calculated by integrating the saturation curve obtained in Figure 4.4 above, and is shown in Figure 4.6.

The above theory is capable of explaining the gas fingering and adsorption observed in the experiments (Brigham el al., 1989). However, this series of calculations is still in the category of Buckley-Leverett theory, whereas the foam flow data does not quite follow that theory. So these calculations do not explain why experimental saturation histories stretch differently at different locations.

All of this theory assumes that no dispersion, or mixing occurs beyond that defined by Buckley-Leverett displacement. The effect of mixing is discussed in the following section.

\subsection{The Effect of Mixing}

From the experimental data, it was observed that the gas saturation profiles stretched to a different degree depending on the location (or distance). This is different from BuckleyLeveret displacement where the saturation profile doubles its length when it doubles the distance, or volume injected. The cause of this behavior is presumed to be the mixing that takes place between the gas (actually foam) and the in-situ fluid. So it was decided that the 


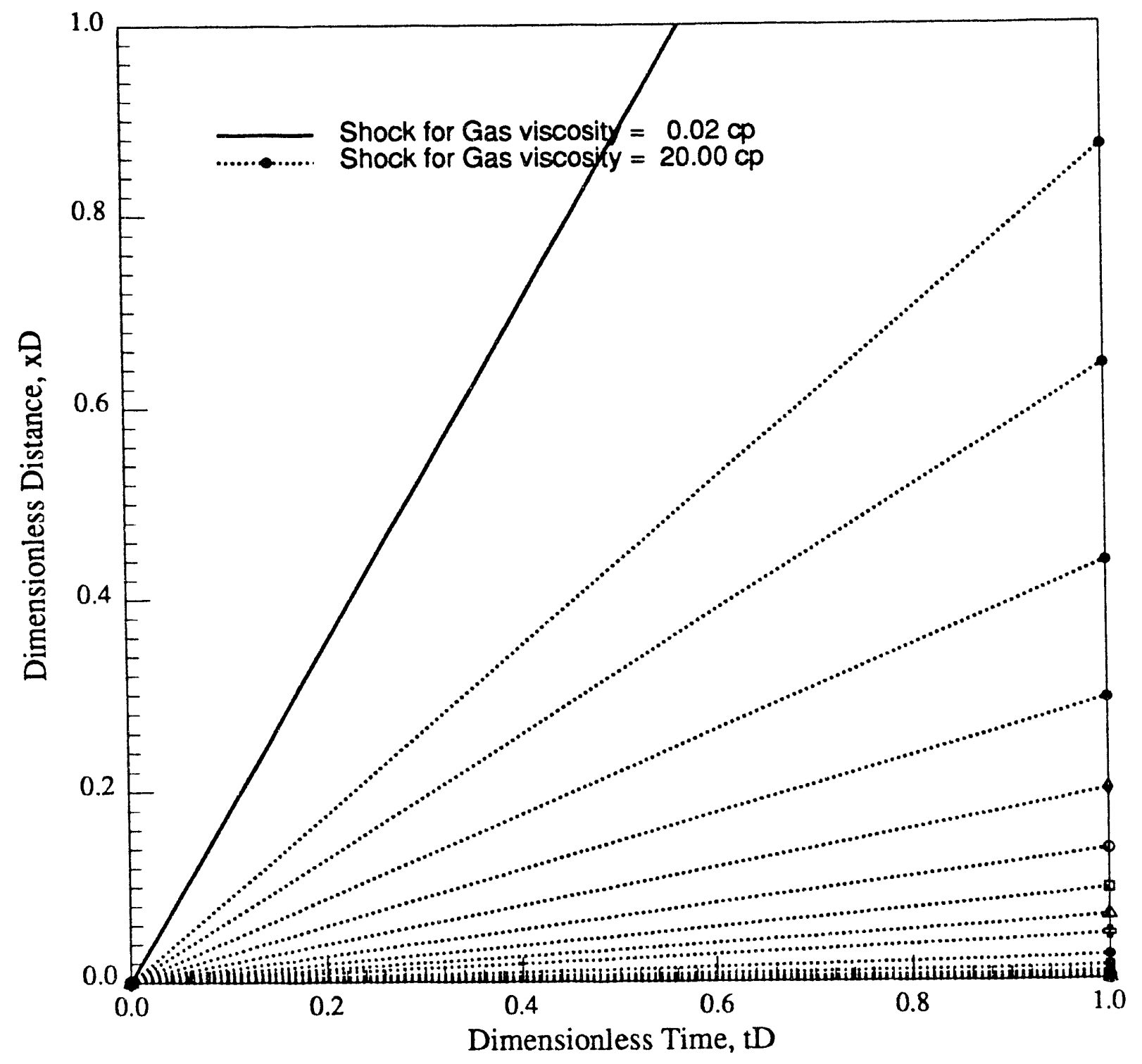

Figure 4.3: Shock Velocities for 20 Shocks (Example) 


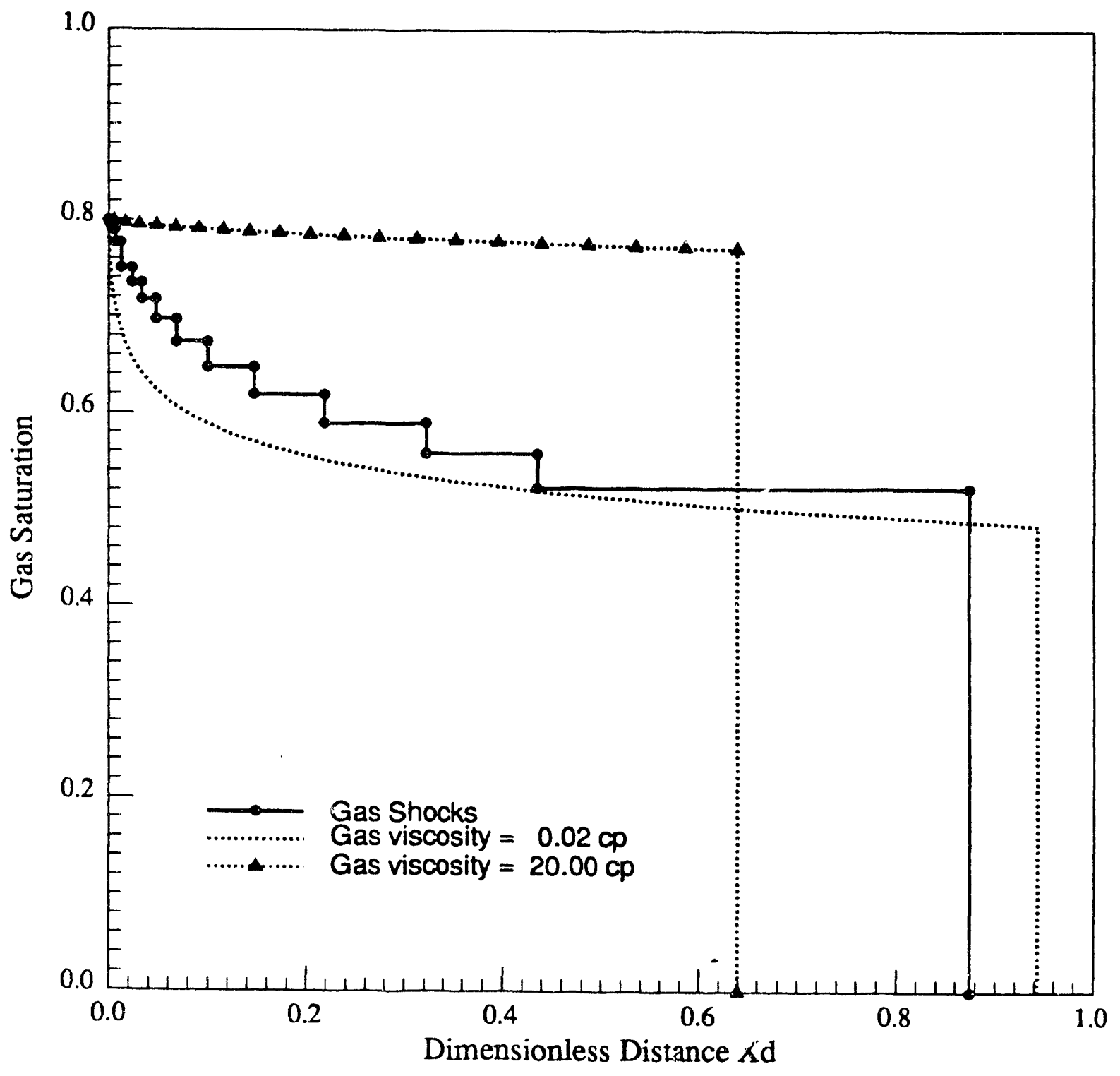

Figure 4.4: Saturation Profiles for 20 Shocks (Example) 


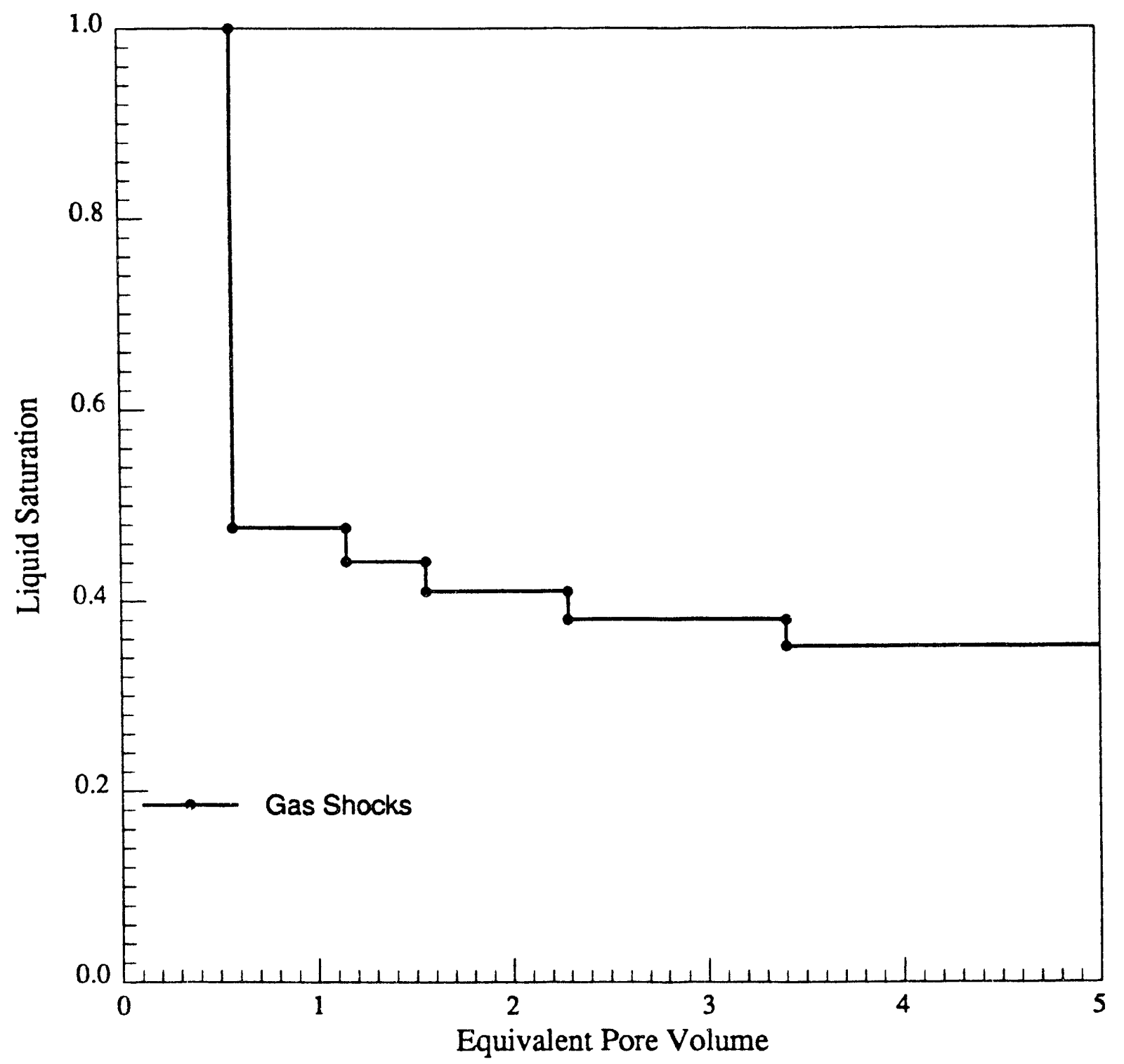

Figure 4.5: Liquid Saturation Profile on an EPV Basis (Example) 


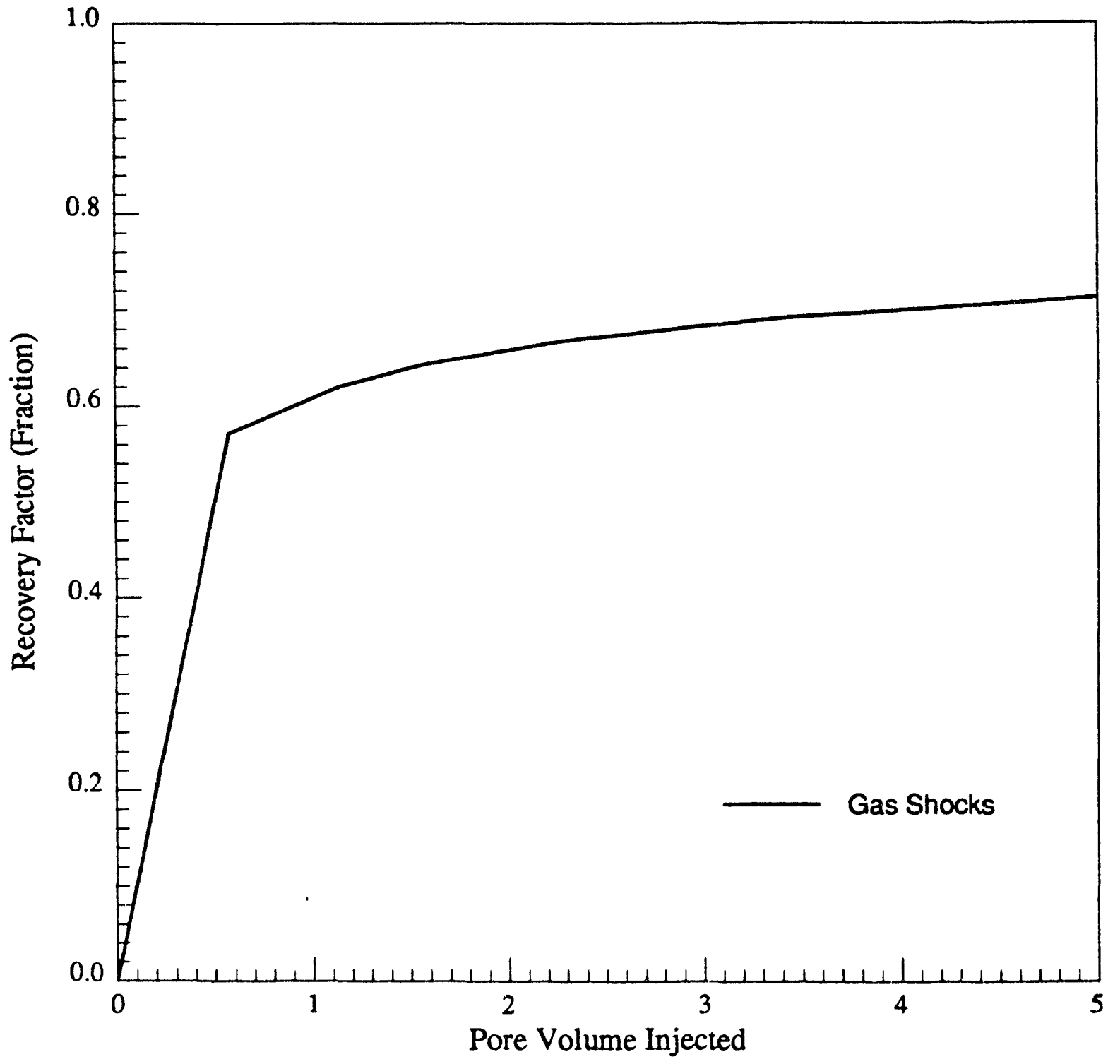

Figure 4.6: Recovery Efficiency History (Example) 36 
displacement could be defined as a combination of pure Buckley-Leverett flow plus mixing.

In flow through porous media, the mixing characteristics of flowing fluids are well known (Liu, 1986; Brigham et al., 1961; Brigham, 1974). Brigham (1986) generalized one of the radial flow approximation methods to various geometries. The following procedure is based largely on the concepts discussed in his 1986 paper.

In the case where mixing is the only spreading mechanism, the standard deviation for linear flow is defined as:

$$
\sigma_{m}^{2}=2 \alpha x
$$

In the case of Buckley-Leverett theory, the standard deviation for linear flow is:

$$
\sigma_{B L}=C x
$$

In the above two equations,

$$
\begin{aligned}
\sigma_{m} & =\text { standard deviation due to mixing, } \mathrm{ft} . \\
\sigma_{B L} & =\text { standard deviation due to Buckley-Leveret theory, } \mathrm{ft} . \\
\alpha & =\text { mixing constant depending on the porous medium, } \mathrm{ft} \\
x & =\text { linear distance traveled by front, or any arbitrarily defined saturation, } \mathrm{ft} \\
C & =\text { constant, dimensionless. }
\end{aligned}
$$

To get the combination of the effects of mixing and pure Buckley-Leverett displacement, Equations 4.11 and 4.12 are differentiated and added together assuming that the total mixing is the sum of the effects for the general case:

$$
d \sigma=d \sigma_{m}+d \sigma_{B L}=\frac{\alpha d x}{\sigma}+C d x
$$


It is this addition of differentials that is similar in concept to the theory in the 1986 Brigham paper. Rearranging Equation 4.13, we get:

$$
d \sigma=\left(\frac{\alpha}{\sigma}+C\right) d x
$$

Integrating Equation 4.14,

$$
\int_{0}^{\sigma} \frac{d \sigma}{(\alpha / \sigma+C)}=\int_{0}^{x} d x
$$

results in the following equation:

$$
\frac{\sigma}{C x}=\frac{1}{1-\frac{\alpha}{C \sigma} \ln \left(1+\frac{C \sigma}{\alpha}\right)}
$$

Equation 4.16 is the theoretical representation of the total mixing effect expected in foam displacement. However, as our purpose was to find a simple solution, an effort was made to simplify this equation further to put it into an algebraic form.

Using the least squares method, a good fit was found to match the theoretical solution (Equation 4.16) over four cycles on the log scale, which is a large enough range for practical purposes for field scale displacements. This fit is hereafter called the semi-analytical mixing equation and is shown in Equation 4.17.

$$
\frac{\sigma}{C^{\prime} x}=1.04675+2.01029\left(\frac{\alpha}{C \sigma}\right)
$$

The comparison of the theoretical mixing curve and the semianalytical mixing curve is shown in Figure 4.7. As can be seen, the fit is generally satisfactory. The constants, $\alpha$ and $C$, can be evaluated from the saturation profiles measured in the experiments, as will be discussed later.

An error analysis was performed for Equations 4.16 and 4.17 and the results are shown in Table 4.1. The maximum error was about $7.5 \%$. 


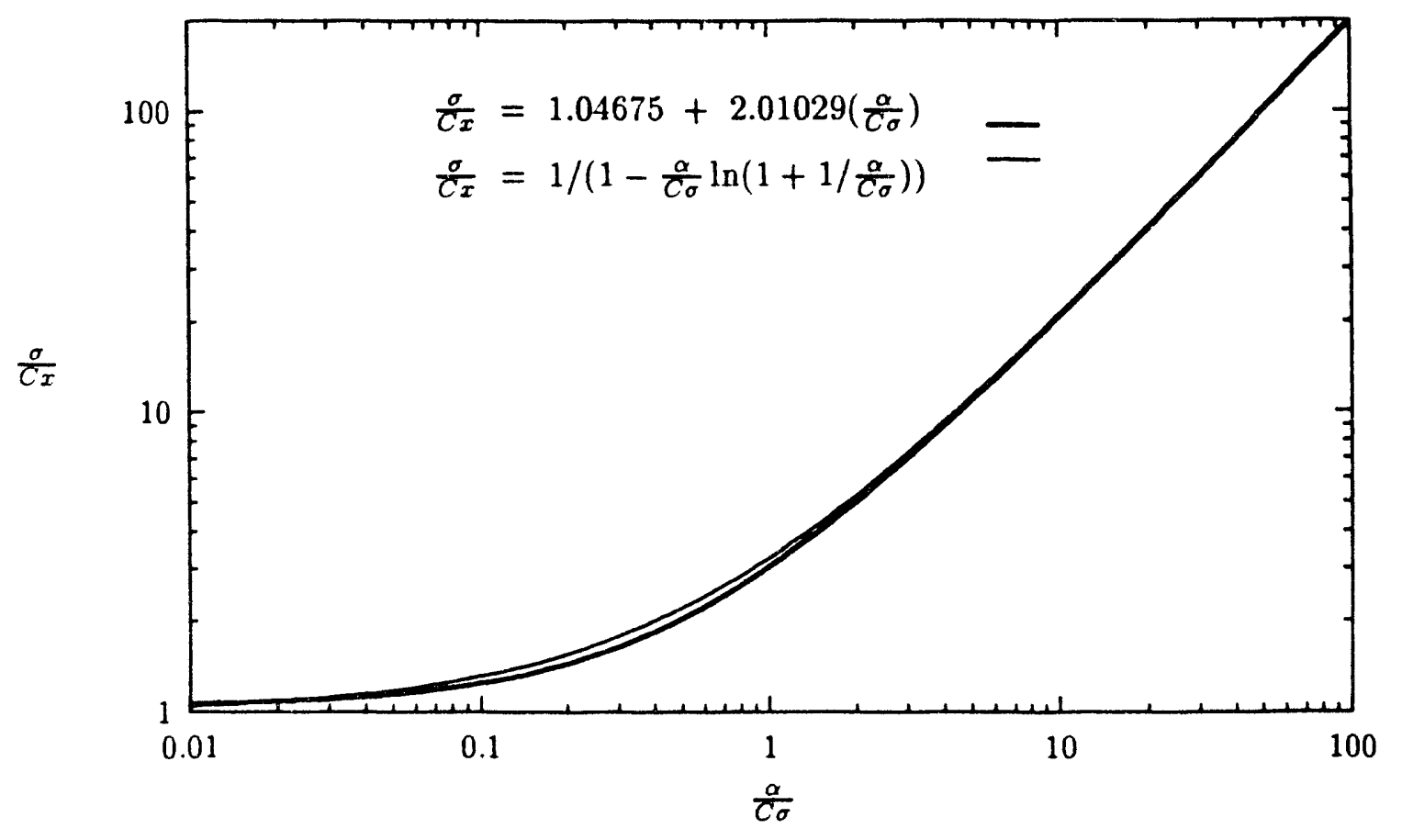

Figure 4.7: Comparison of Mixing Curves 


\begin{tabular}{|r|r|r|r|}
\hline \multirow{2}{*}{$\frac{\alpha}{C \sigma}$} & \multicolumn{2}{|c|}{$\frac{\sigma}{C r}$} & $\begin{array}{r}\text { Relative } \\
\text { Error (\%) }\end{array}$ \\
\cline { 2 - 3 } & Eq. 4.16 & Eq. 4.17 & +1.76 \\
\hline 0.01 & 1.04838 & 1.06685 & +0.14 \\
\hline 0.02 & 1.08535 & 1.08696 & 2.73 \\
\hline 0.05 & 1.17956 & 1.14726 & -5.14 \\
\hline 0.1 & 1.31543 & 1.24778 & -7.03 \\
\hline 0.2 & 1.55849 & 1.44881 & -7.52 \\
\hline 0.5 & 2.2188 & 2.0519 & -6.19 \\
\hline 1.0 & 3.25889 & 3.05704 & -4.19 \\
\hline 2.0 & 5.28905 & 5.06733 & -1.90 \\
\hline 5.0 & 11.3132 & 11.0982 & -0.81 \\
\hline 10.0 & 21.3228 & 21.1497 & -0.18 \\
\hline 20.0 & 41.3279 & 41.2526 & +0.22 \\
\hline 50.0 & 101.331 & 101.561 & +0.36 \\
\hline 100. & 201.332 & 202.076 & +0.36 \\
\hline
\end{tabular}

Table 4.1: Error Analysis of Brigham Type Curve (Large Range of $\alpha / C \sigma$ )

It was noted that the experimental data in this work fell between the range of 0.05 and 5 for $a / C \sigma$ where the errors were the greatest. So the constants in Equation 4.17 were modified slightly to fit a smaller range with greater accuracy. The constants were found to be 1.123 and 2.067, as shown in Equation 4.18

$$
\frac{\sigma}{C x}=1.123+2.067\left(\frac{\alpha}{C \sigma}\right)
$$

Another error analysis performed in Table 4.2 indicates that greater accuracy (within $4 \%$ ) could be achieved for this range.

To show how this equation is used, the semi-analytical equation can be impiemented as illustrated in Figure 4.8. In this figure, there are two saturation profiles at two different times ( 0.2 and 0.5 pore volumes injected). An arbitrary saturation range from 0.50 to 0.58 was chosen to define the width of the mixed zone, $\sigma$. In general, any fixed range can be used, for the shape of the saturation profile is assumed to be fixed. Only its overall length changes with distance, not its shape. This saturation difference resulted in the distance $\Delta x_{1}$ at $0.2 \mathrm{PV}$ injected. The average saturation, $\bar{S}_{g}$, has traveled a distance $\bar{X}_{1}$. Note here that $\Delta X_{1}$ is linearly proportional to the standard deviation $(\sigma)$ of the average saturation, while $\bar{X}_{1}$ is proportional to and corresponds to the distance $x$ in Equations 4.16 and 4.17 . At a later time (0.5 PV injected), the same saturations chosen before are distributed over 


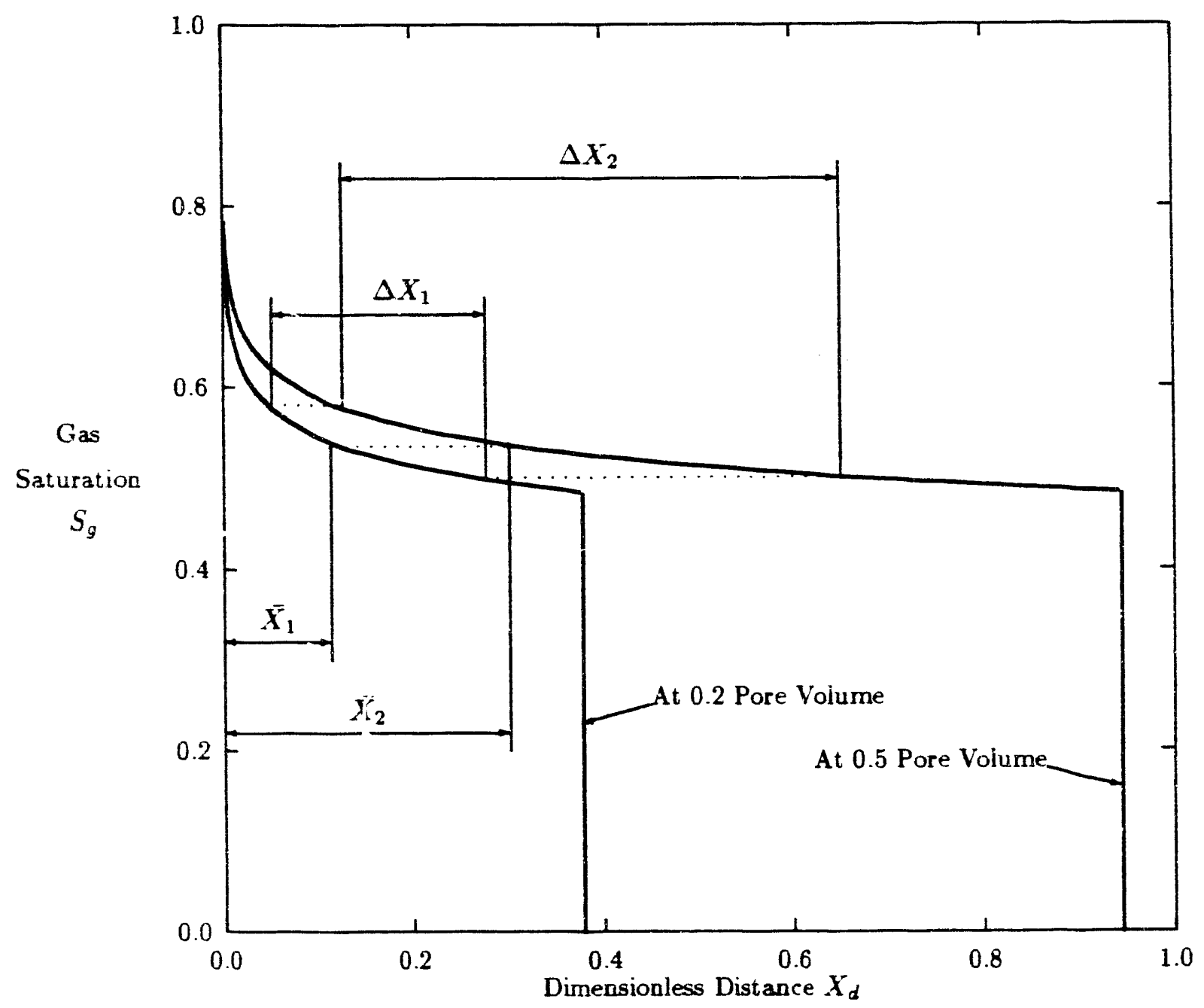

Figure 4.8: Semi-Empirical Mixing $E_{\text {jquation Implementation }}$ 


\begin{tabular}{|r|r|r|r|}
\hline \multirow{2}{*}{$\frac{\alpha}{C \sigma}$} & \multicolumn{2}{|c|}{$\frac{\sigma}{C_{T}}$} & $\begin{array}{r}\text { Relative } \\
\text { Error (\%) }\end{array}$ \\
\cline { 2 - 3 } & Eq. 4.16 & Eq. 4.18 & +3.9 \\
\hline 0.05 & 1.17956 & 1.226 & +3.2 \\
\hline 0.06 & 1.20817 & 1.247 & +2.0 \\
\hline 0.08 & 1.26297 & 1.288 & +1.1 \\
\hline 0.1 & 1.31543 & 1.330 & -1.4 \\
\hline 0.2 & 1.55849 & 1.536 & -2.4 \\
\hline 0.3 & 1.7854 & 1.743 & -2.8 \\
\hline 0.6 & 2.43012 & 2.363 & -2.5 \\
\hline 0.8 & 2.84693 & 2.777 & 2.1 \\
\hline 1.0 & 3.25889 & 3.190 & -0.6 \\
\hline 2.0 & 5.28905 & 5.257 & +0.3 \\
\hline 3.0 & 7.30173 & 7.324 & +1.3 \\
\hline 5.0 & 11.3132 & 11.458 & + \\
\hline
\end{tabular}

Table 4.2: Error Analysis of Brigham Type Curve ( Small Range of $\alpha / C \sigma$ )

the distance of $\Delta X_{2}$ and the average saturation has arrived at $\bar{X}_{2}$. This process is repeated for all the saturation profiles measured at different injection times. Thus a set of numbers, $\Delta X / \bar{X}$ vs. $1 / \Delta X$ (which is equivalent to $\sigma / x$ vs. $1 / \sigma$ ), are obtained for each saturation profile. These points from all the saturation profiles can then be graphed on log-log paper in the same manner as the theoretical equation in Figure 4.7.

The semi-analytical mixing equation (Eq. 4.18) is plotted separately in Figure 4.9 and is referred to hereafter as the Brigham Type Curve. The constants, $C$ and $\alpha$, could be found simply by matching the data points plotted on the log-log paper with the type curve. However, for our purposes here, a computer program was written to do the matching, and is listed in Appendix D.

Once $C$ and $\alpha$ are found from the experiments, they can be used in simulations to predict saturation distributions, as a function of distance and volume injected.

\subsection{Summary of the Theory}

Briefly the fractional fiow curve construction method can be summarized in to the following simiple equations. 


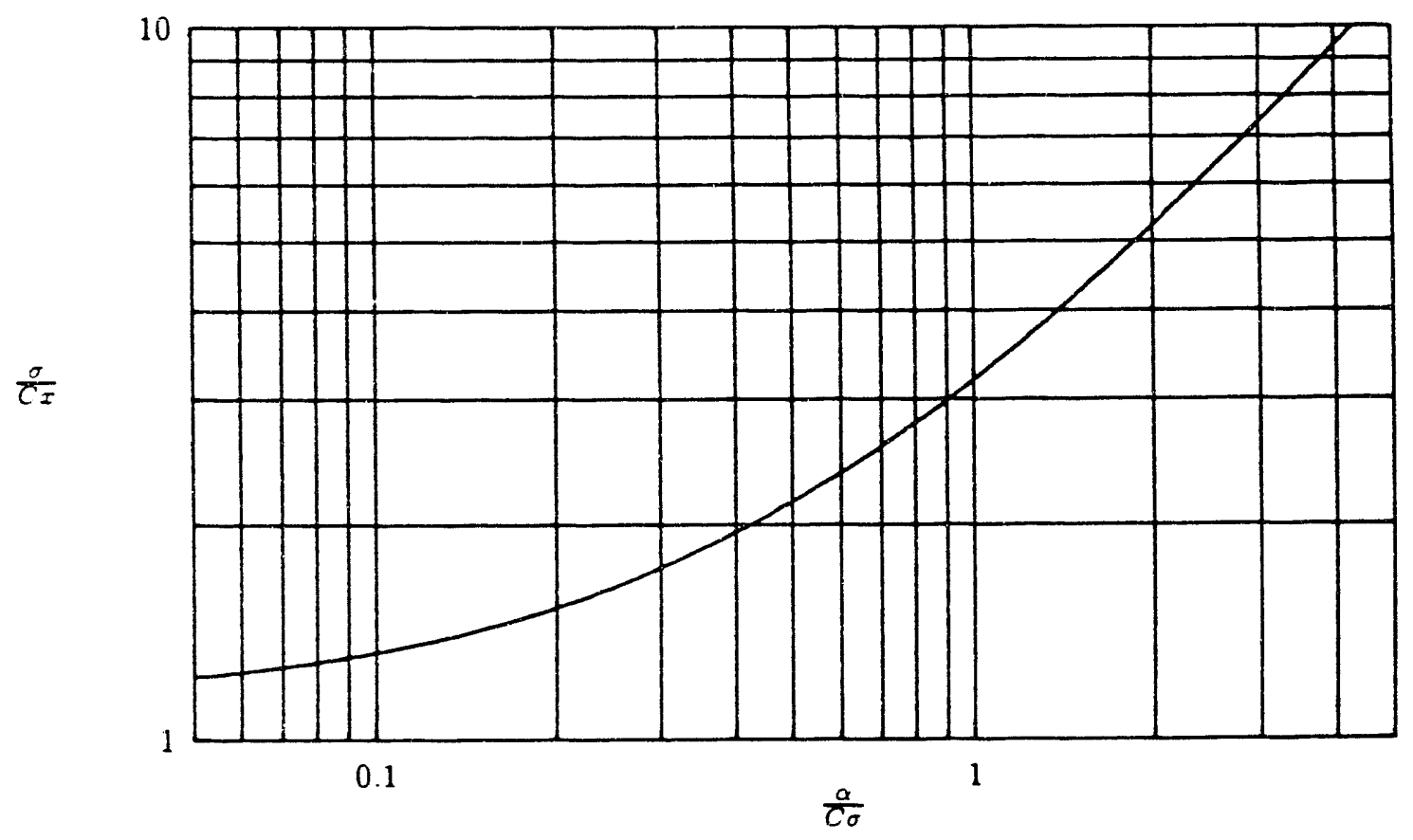

Figure 4.9: Type Curve Combining Mixing with Buckley-Leverett Theory 
To get the first gas shock velocity:

$$
v_{1}=\frac{f_{g_{1}}-f_{g_{0}}}{S_{g_{1}}+D_{1}}=f_{g_{1}}^{\prime}
$$

where the adsorption term, $D_{1}$ is:

$$
D_{1}=\frac{a}{1+b C_{1}}
$$

To get the $n$th gas shock velocity $(n \geq 2)$ :

$$
v_{n}=\frac{f_{g_{n}}-f_{g_{n-1}}}{\left(S_{g_{n}}+D_{n}\right)-\left(S_{g_{n-1}}+D_{n-1}\right)}=f_{g_{n}}^{\prime}
$$

and the adsorption terms are:

$$
\begin{aligned}
D_{n} & =\frac{a}{1+b C_{n}} \\
D_{n-1} & =\frac{a}{1+b C_{n-1}}
\end{aligned}
$$

The mixing effect is reflected in the following equations:

$$
\frac{\sigma}{C x}=\frac{1}{1-\frac{a}{C \sigma} \ln \left(1+\frac{C \sigma}{a}\right)}
$$

which can be well approximated over the entire range of: $0.01 \leq \alpha / C \sigma \leq 100$, by

$$
\frac{\sigma}{C x}=1.04675+2.01029\left(\frac{\alpha}{C \sigma}\right)
$$


For greater accuracy over a smaller range, the constants, 1.04675 and 2.01029 can be changed slightly, as indicated by Equation 4.18.

In Equations 4.16, 4.17 and 4.18 , the constants $C$ and $\alpha$ can be obtained by type curve matching and used to calculate the saturation profiles as a function of distance, and pore volume in jected. 


\section{Chapter 5}

\section{Results for Gas Displacements}

This chapter presents preliminary data gathered and processed to obtain the necessary parameters for subsequent matching of the foam flow behavior. To this end, two series of runs were made based on trial foam runs that had been made previously and the proposed theory for foam flow. The purpose of these preliminary experiments was two-fold: to find the fractional flow curves for various surfactant concentrations (and hence various viscosities), and to find the adsorption behavior. In the first series of experiments, gas was injected into a sandpack saturated with surfactant solutions of various concentrations, ranging from 0 to $1 \%$. These experiments gave rise to the fractional flow curves at different but fixed concentrations.

The second series of experiments was designed to measure the amount of surfactant adsorbed onto the sand surface during displacement. Surfactant solutions of different concentration were injected into a sandpack saturated with distilled water. The effluent fluid was collected and analyzed using the Hyamine dye titration method (Appendix A) to obtain the produced surfactant concentrations, and thus to calculate adsorption.

In the following, the experimental results and observations are discussed first. Theoretical and numerical investigations are presented in a later section (Section 5.3).

\subsection{Displacements at Various Surfactant Concentrations}

For each cross section during the process of the displacement experiments at various fixed concentrations, a series of scans were taken at different stages and the CT numbers were obtained from the CT computer. From these scans, the liquid saturations were calculated 
using Equation ??. It should be noted that the CT numbers given are the mean values. There was a standard deviation attached to each CT number. A greater standard deviation means a more heterogeneous saturation distribution in a cross section.

To obtain the fractional flow curves for various gas (foam) viscosities, the first series of experiments were run at various surfactant concentrations. The important parameters for these runs are summarized in Table 5.1. In each run, the saturations were measured at several fixed locations in the sandpacks. Then the saturations were plotted against Equivalent Pore Volumes (EPV) injected. These saturation histories at different locations should fall on top of each other if the displacements follow the Buckley-Leverett theory. Pressure above the backpressure were recorded at the pressure taps along the sandpacks. Also, the effluent fluids were collected to obtain the recovery history. These sets of data were used to double-check the validity and consistency of the results and often led to more accurate answers.

\begin{tabular}{|c|c|c|c|c|c|}
\hline $\begin{array}{c}\text { Run } \\
\text { No. }\end{array}$ & $\begin{array}{c}\text { Injection } \\
\text { Velocity (cc/min) }\end{array}$ & $\begin{array}{c}\text { Surfactant } \\
\text { Concentration }(w t \%)\end{array}$ & $\begin{array}{c}\mathrm{k} \\
(\text { Darcies })\end{array}$ & $\begin{array}{c}\phi \\
(\%)\end{array}$ & $\begin{array}{c}\text { Length of } \\
\text { Sandpack }\end{array}$ \\
\hline 21 & 0.7333 & 0.0 & 6.7 & 32 & 46 inches \\
\hline 31 & 0.9704 & 0.001 & 6.7 & 32 & 24 inches \\
\hline 32 & 1.0110 & 0.01 & 6.7 & 32 & 24 inches \\
\hline 35 & 0.8962 & 0.1 & 6.7 & 32 & 46 inches \\
\hline 34 & 0.7750 & 1.0 & 6.7 & 32 & 24 inches \\
\hline
\end{tabular}

Table 5.1: Experimental Runs of Gas Displacement

To begin the series of experiments, pure nitrogen was injected into a sandpack saturated initially with distilled water without surfactant. The purpose of this run was to generate the exponents of relative permeabilities in Eqs 4.1 and 4.2 for the gas-water system and to verify the application of Buckley-Leveret theory in this system. As shown in Figure 5.1 , the liquid saturation at different locations $(4.7,14.7,30.5$ inches from the inlet) were plotted versus the EPV injected. As can be seen from the figure, the sat uration histories did indeed fall nearly on top of each other within a satisfactory degree. This indicates that this displacement followed the Buckley-Leverett theory. The pressure data are shown in Figure 5.2. It can be seen that for such a gas-water system, the pressure behaved erratically.

In Run 31 , gas was injected into a shorter sandpack initially saturated with surfactantladen solution of $0.001 \%$ concentration. The results are shown in Figures 5.3 and 5.4. As can be seen from the figures, the breakthrough time was later than that in R.un 21, in which no surfactant was present. Also the liquid displacement was faster and the pressure drop 


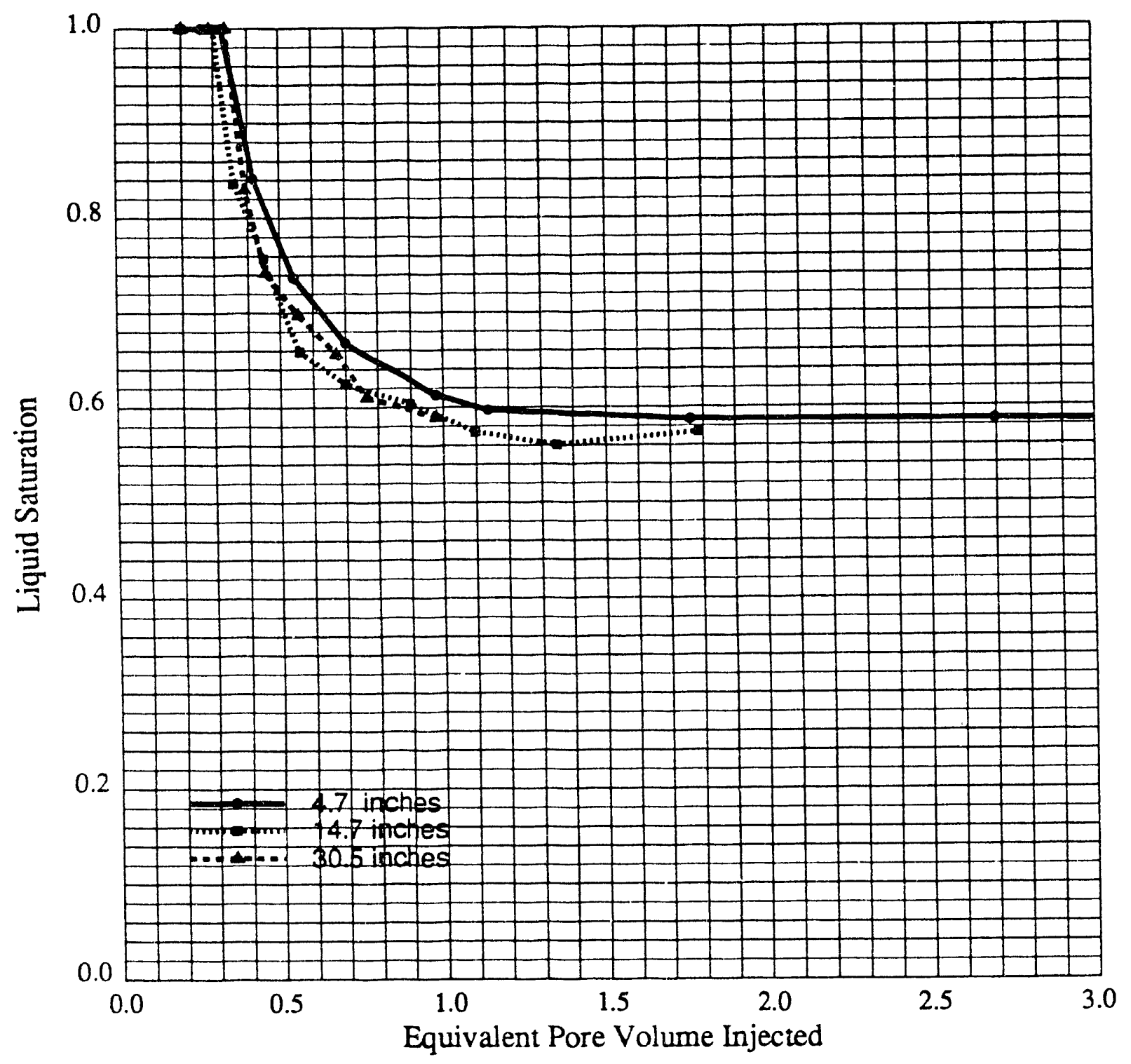

Figure 5.1: Saturation Histories for Run 21 (Gas Displacing Water) 


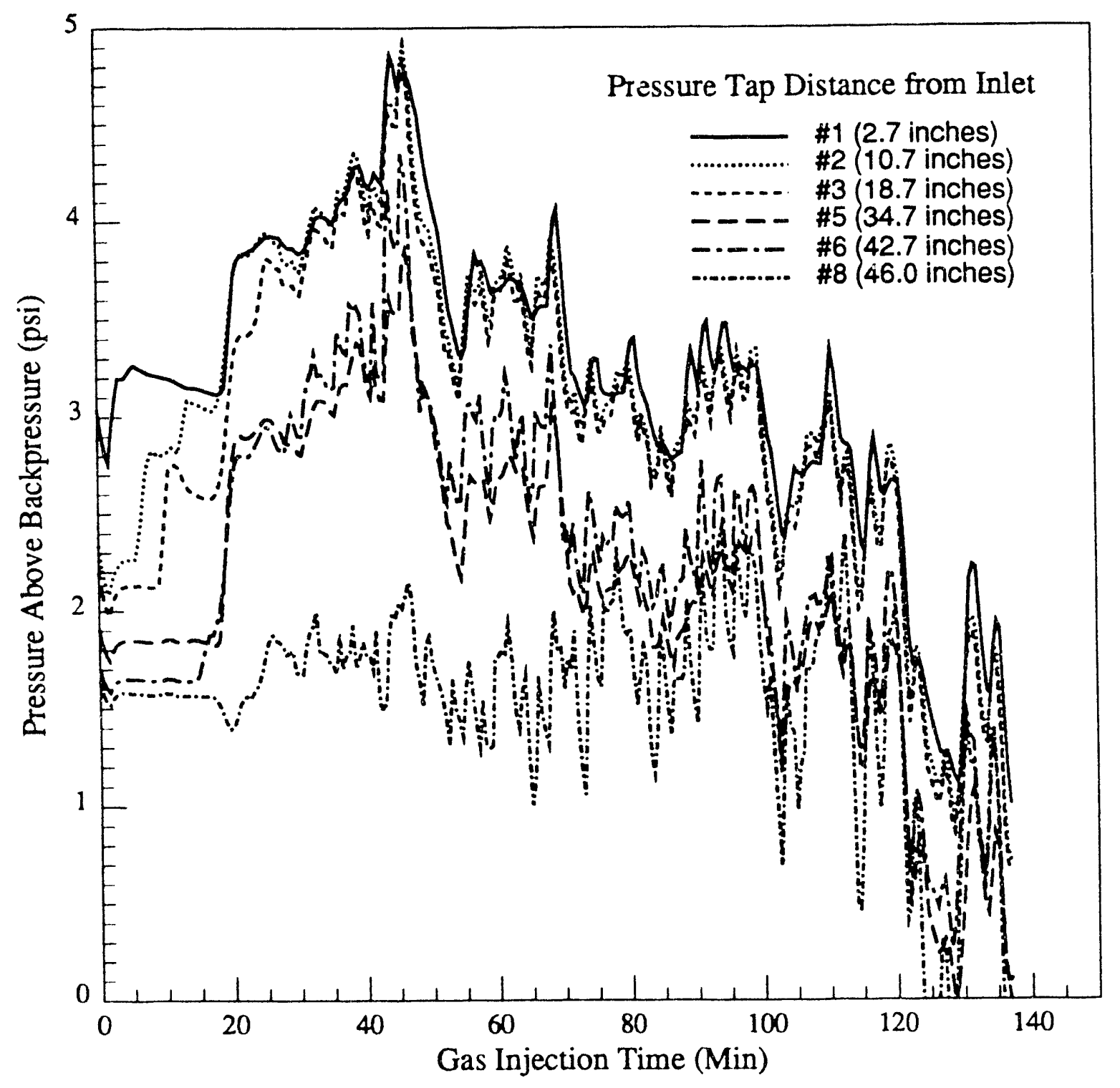

Figure 5.2: Pressure Responses for Run 21 (Gas Displacing Water) 


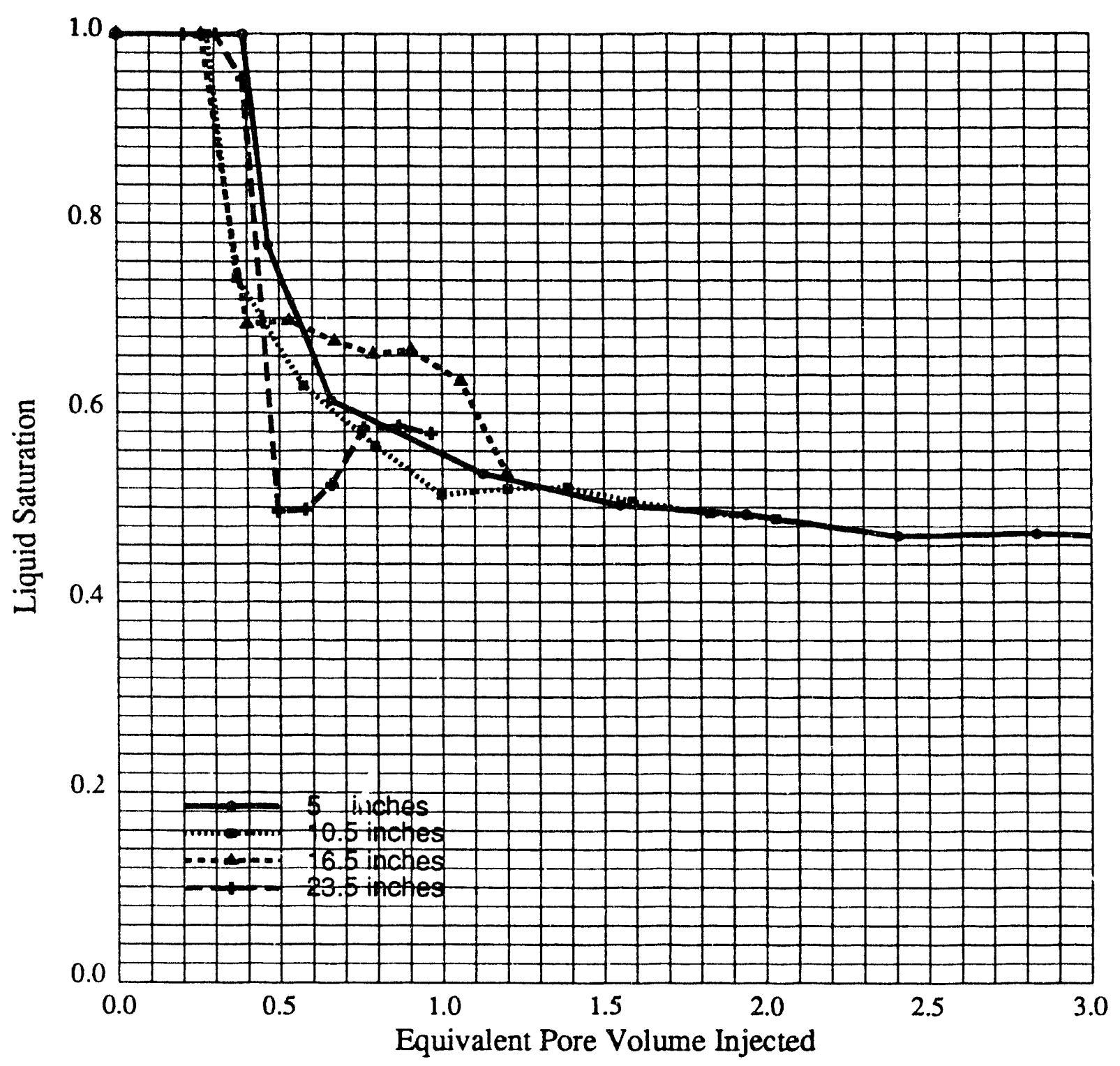

Figure 5.3: Saturation Histories for Run 31 (Gas Displacing 0.001\% Surfactant-Laden Solution) 


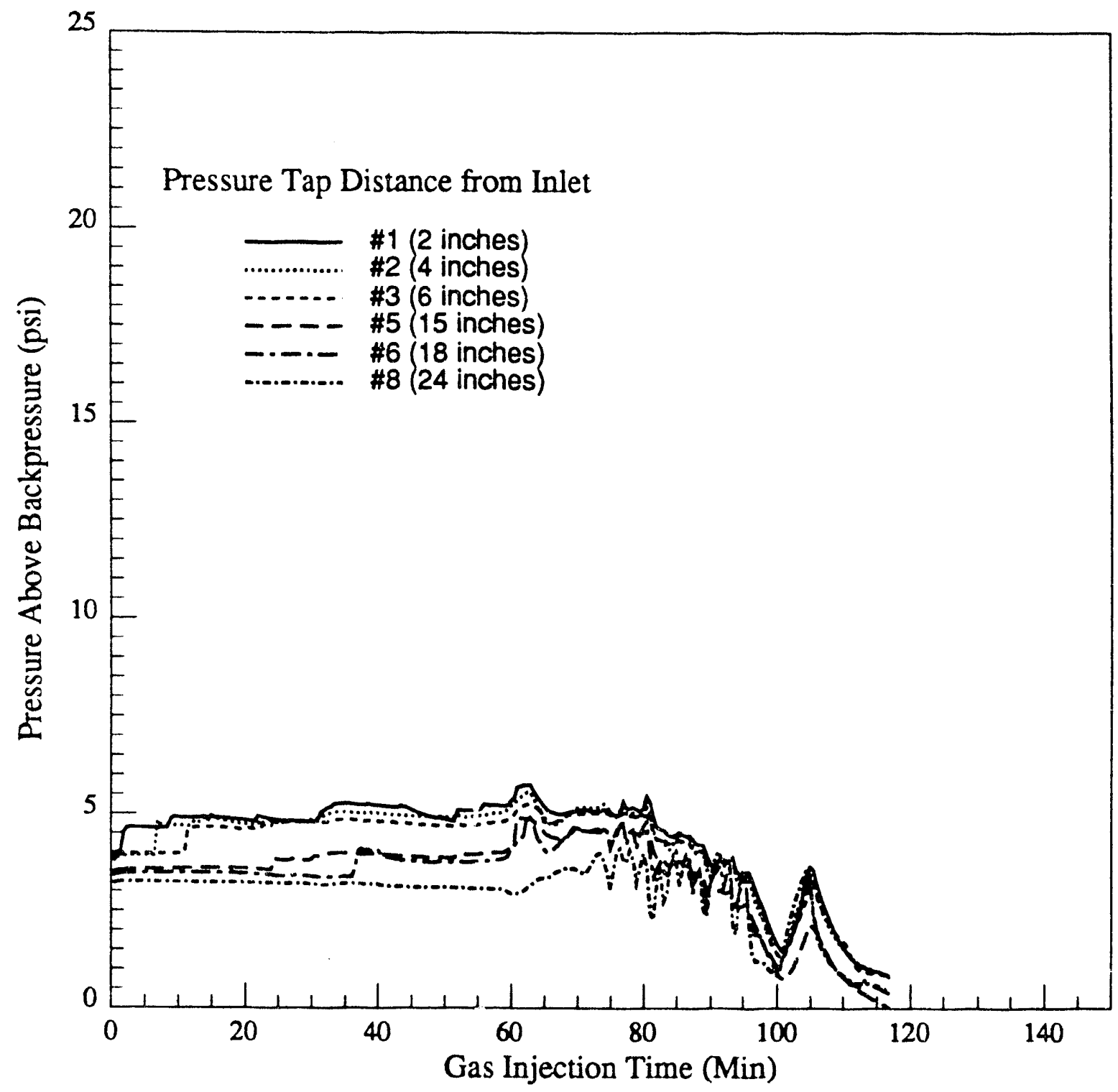

Figure 5.4: Pressure Responses for Run 31 (Gas Displacing 0.001\% Surfactant-Laden Solution) 
higher and less wildly varying than in Run 21 . These results point out a very interesting fact, even a very small concentration of surfactant could have a large effect on the flow behavior.

Another point should be mentioned concerning the saturation distribution curves of Figure 5.3. Note that they are not as smooth or as consistent as those in Figure 5.1. In fact, as will be seen later, they were the most erratic of this series of runs. No physical or experimental reason has been found for these erratic results. Notice that some of the curves increase in liquid saturation or stay relatively flat with time and then decrease again which is contrary to any displacement theory. Overall, however, the curves do not seem to be a constant function of EPV injected, which is consistent with Buckley-Leverett theory.

In $R$ un 32 , the surfactant concentration was increased to $0.01 \%$. As shown in Figures 5.5 and 5.6, the displacement was even more efficient than that in the previous runs. This run suggested a higher apparent viscosity of foam during the displacement, compared to the two previous runs.

Note that the saturation distribution curves of Figure 5.5 do not fall exactly on top of each other, as they should for Buckley-Leverett displacement. Notice, however, that the saturation histories are so sharp near the front that it was very difficult to define their exact shapes. Overall, the results appear to match Buckley-Leverett theory adequately.

The results for Run 33 (gas displacing $0.1 \%$ surfactant-laden solution) in a short sandpack are plotted in Figures 5.7 and 5.8. To verify the reproducibility of the experiments with different sandpacks, Run 35 was made under exactly the same conditions as in Run 33 with a long sandpack. The results, shown in Figures 5.9 and 5.10, demonstrated that the length of the sandpack had little effect on the displacement. This observation also illustrated the effectiveness of the packing method used in this study.

The final experiment in this series (Run 34) in a short sandpack involved the gas displacing surfactant solution of $1 \%$ concentration. As shown in Figures 5.11 and 5.12 , the displacement was the most efficient of the all the runs in this series, as should have been expected, from the results of the other runs.

Looking at all these runs, one can realize that when there is a constant surfactant concentration in a flow system, the displacement follows the Buckley-Leverett theory. There were some factors affecting the accuracy of data acquisition, such as the CAT Scanner itself, time constraints, actual sandpacking, and human errors. Some of the runs gave consistent saturation data (Figure 5.1, Figure 5.9 and Figure 5.11). The saturation histories for Run 31 (Figure 5.3) had some ups and downs, which probably were not realistic, but they still 


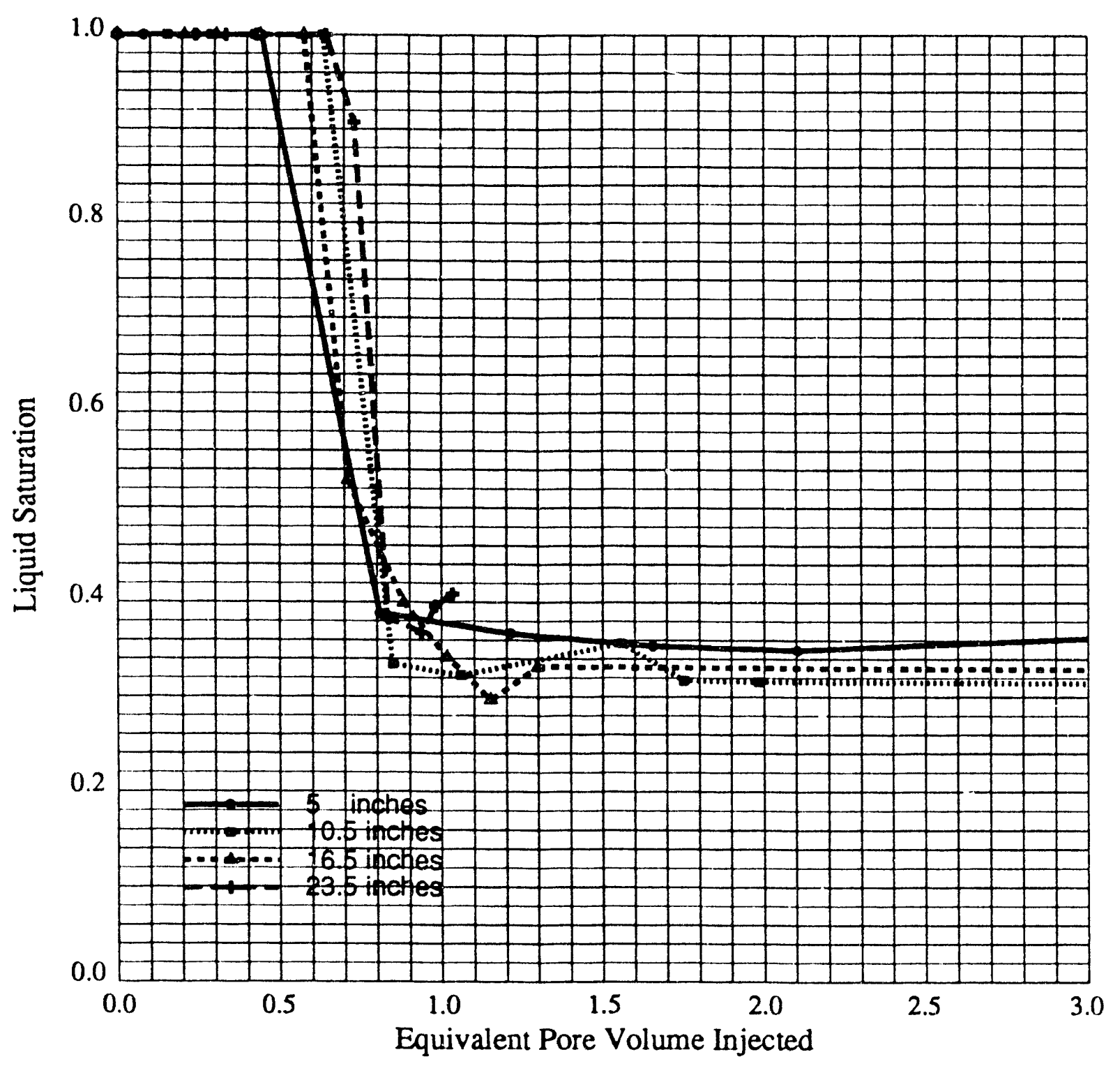

Figure 5.5: Saturation Histories for Run 32 (Gas Displacing 0.01\% Surfactant-Laden Solution) 


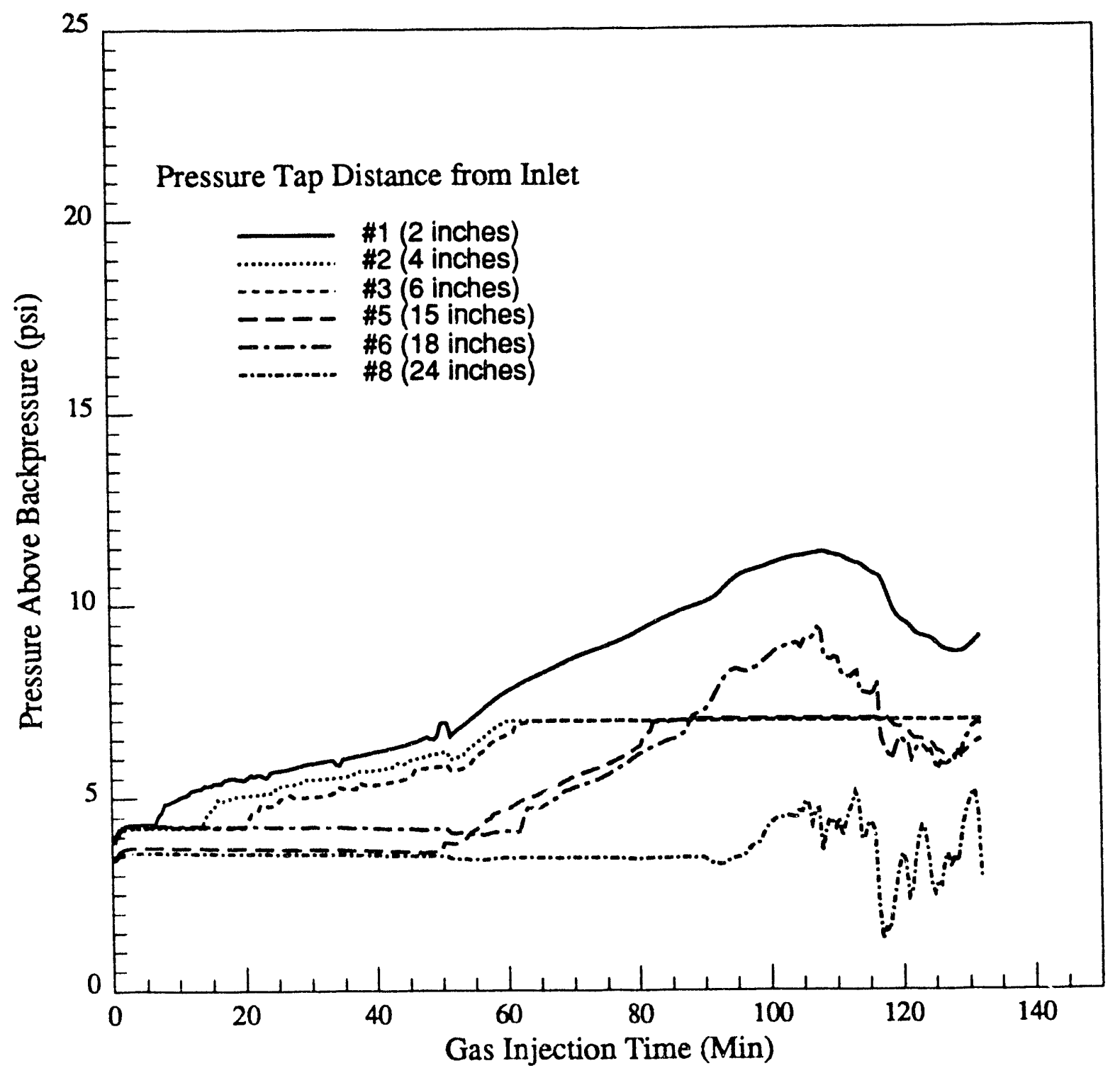

Figure 5.6: Pressure Responses for Run 32 (Gas Displacing 0.01\% Surfactant-Laden Solution) 


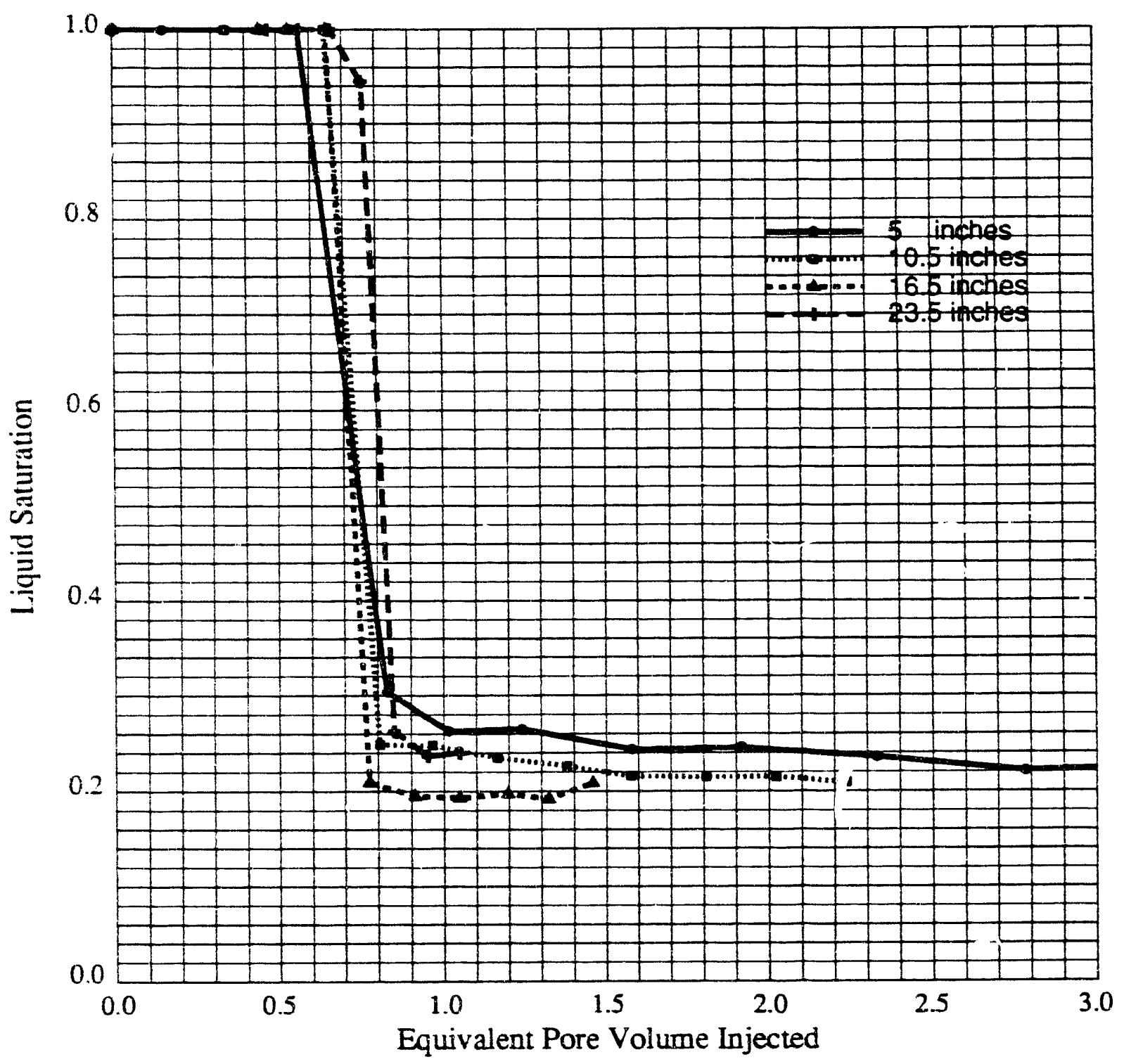

Figure 5.7: Saturation Histories for Run 33 (Gas Displacing 0.1\% Surfactant-Laden Solution : 


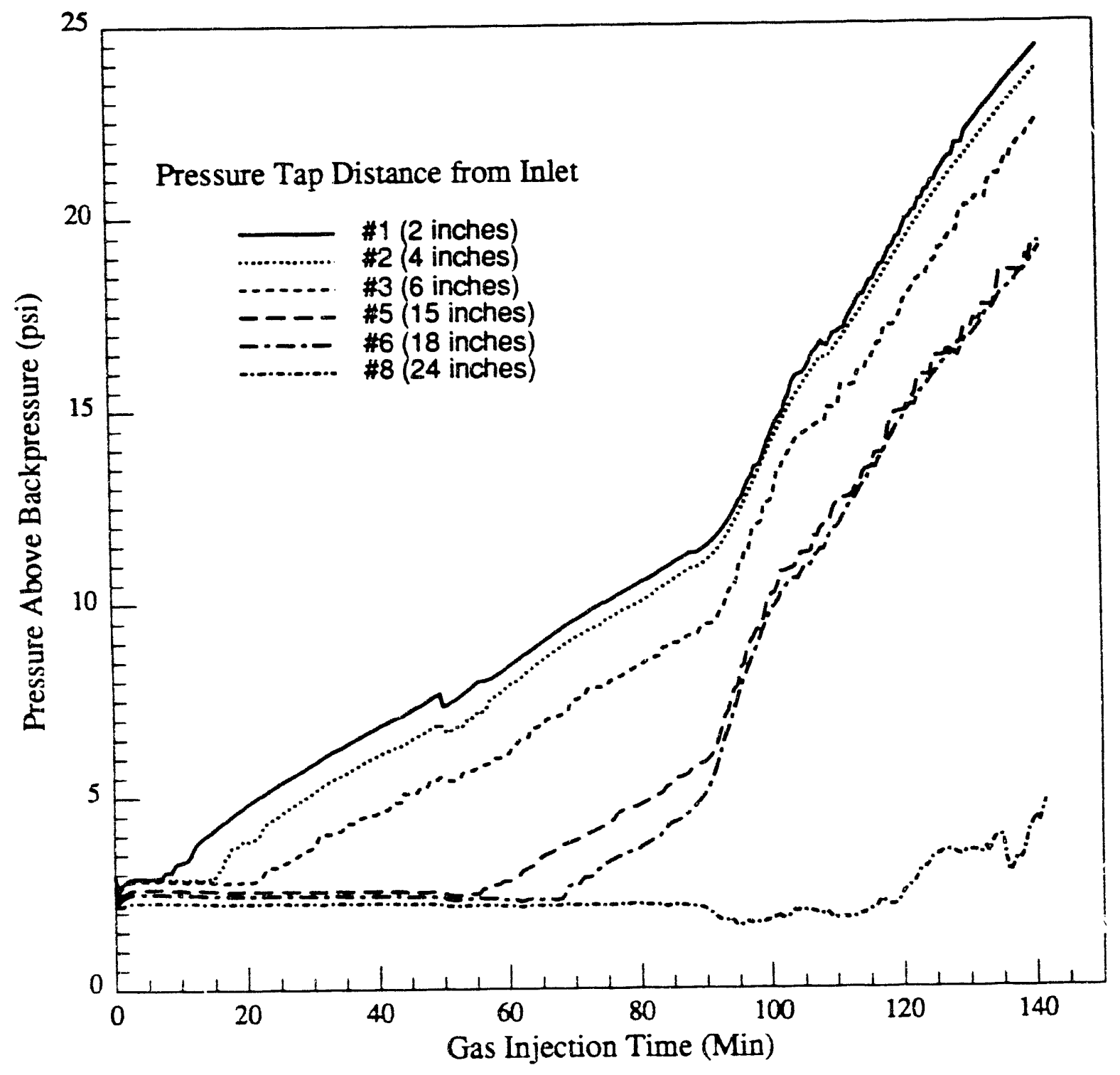

Figure 5.8: Pressure Responses for Run 33 (Gas Displacing 0.1\% Surfactant-Laden Solution) 


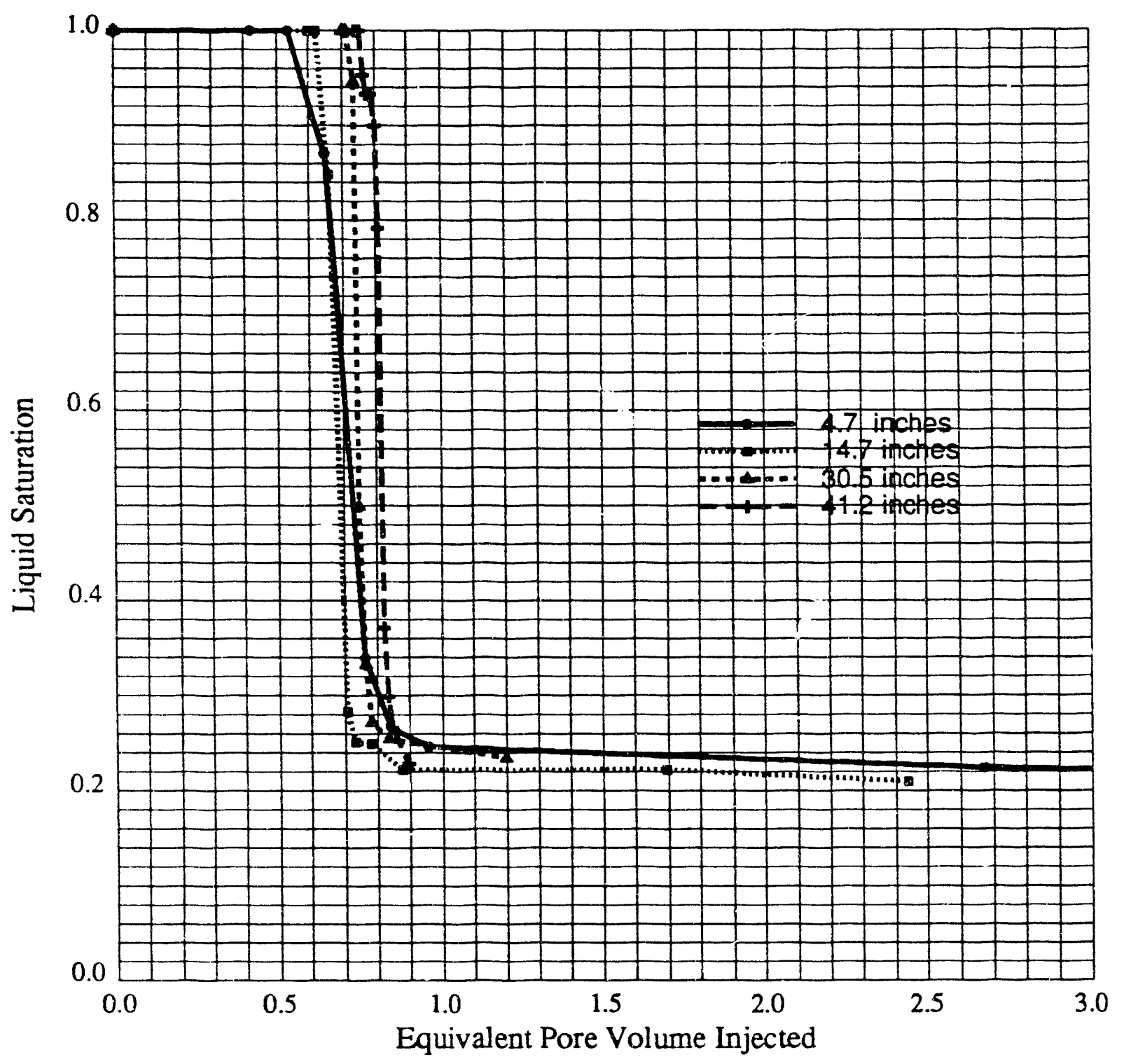

Figure 5.9: Saturation Histories for Run 35 (Gas Displacing 0.1\% Surfactant-Laden Solution) 


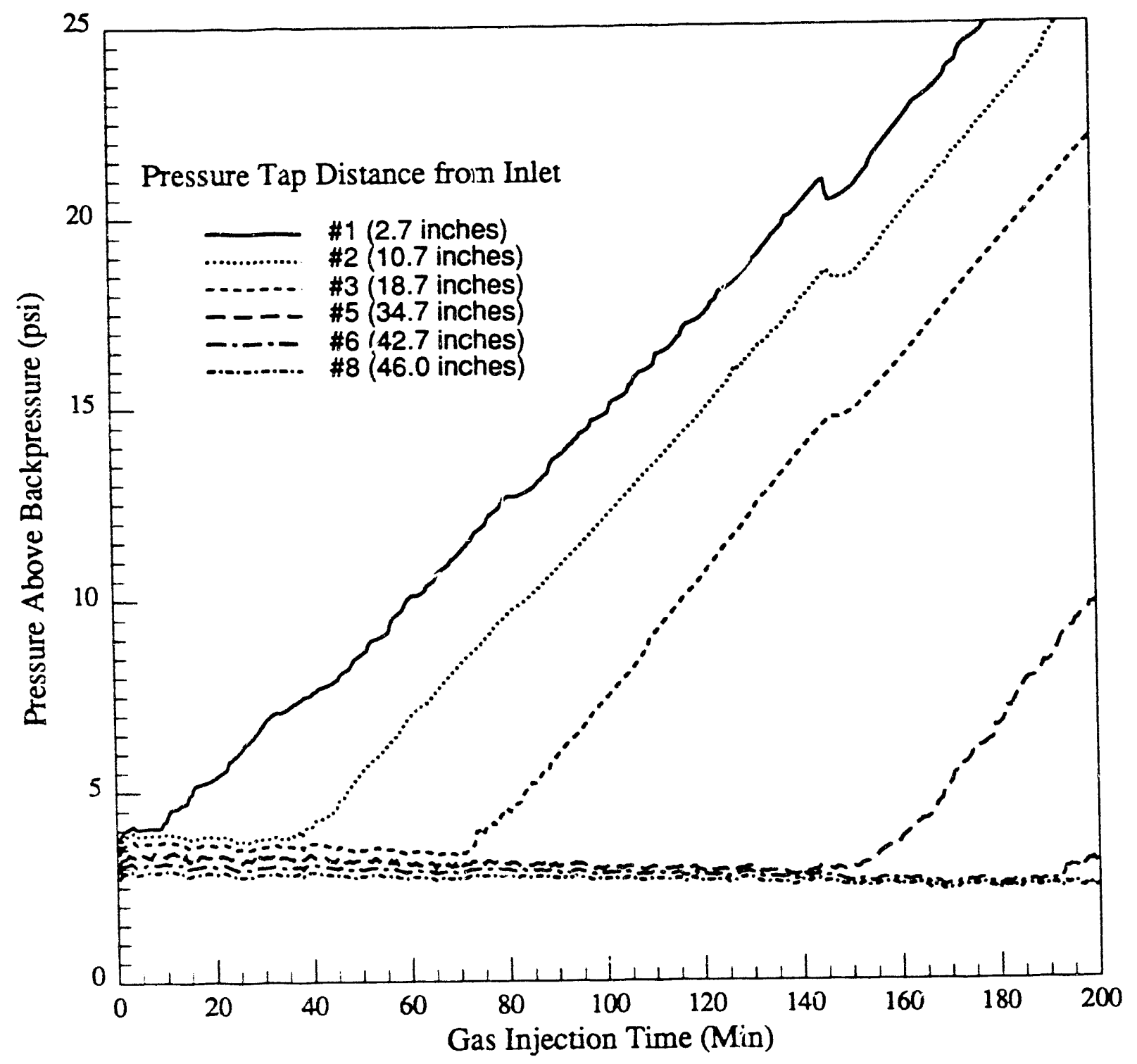

Figure 5.10: Pressure Responses for Run 35 (Gas Displacing 0.1\% Surfactant-Laden Solution) 


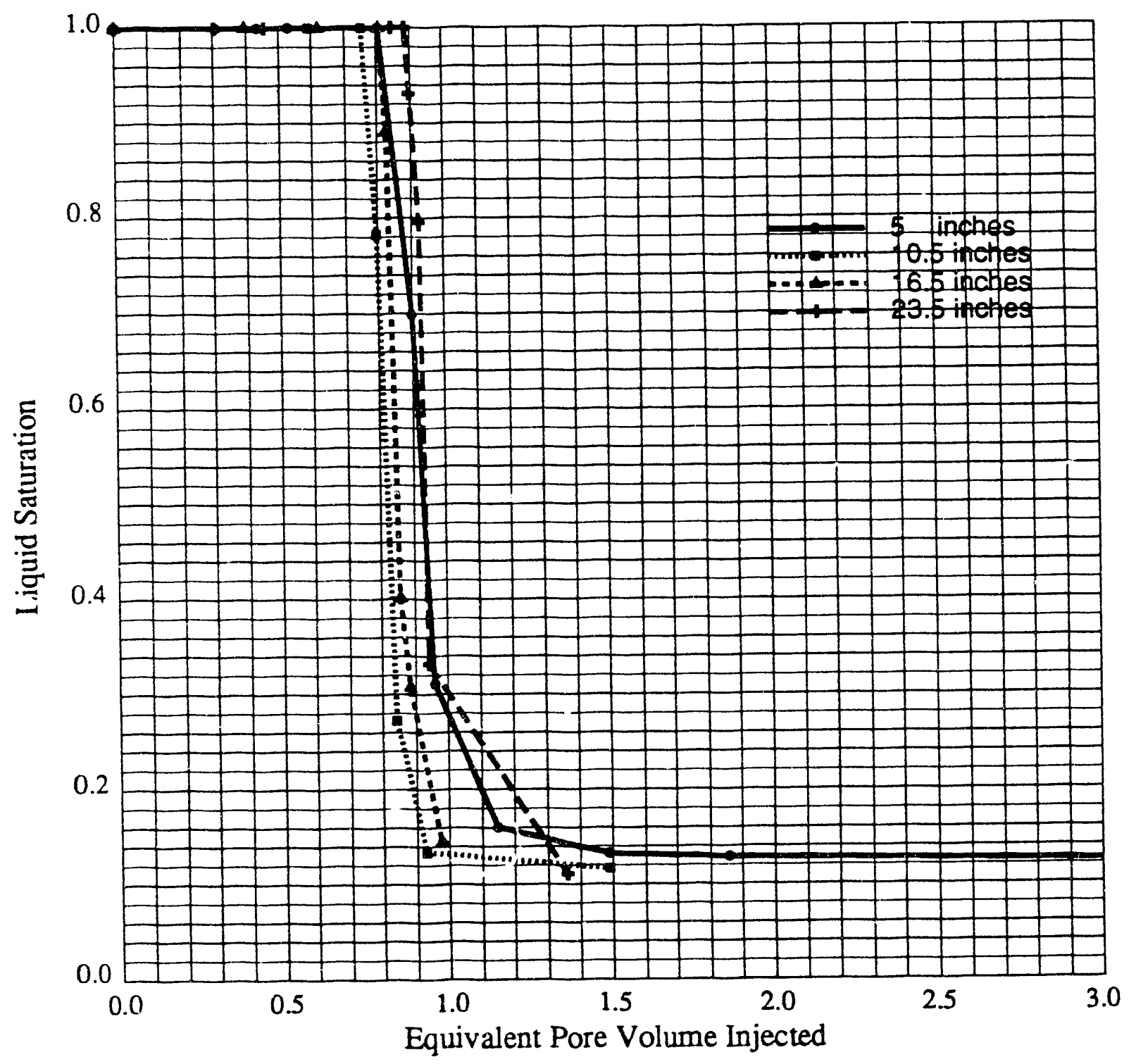

Figure 5.11: Saturation Histories for Run 34 (Gas Displacing 1\% Surfactant-Laden Solution) 


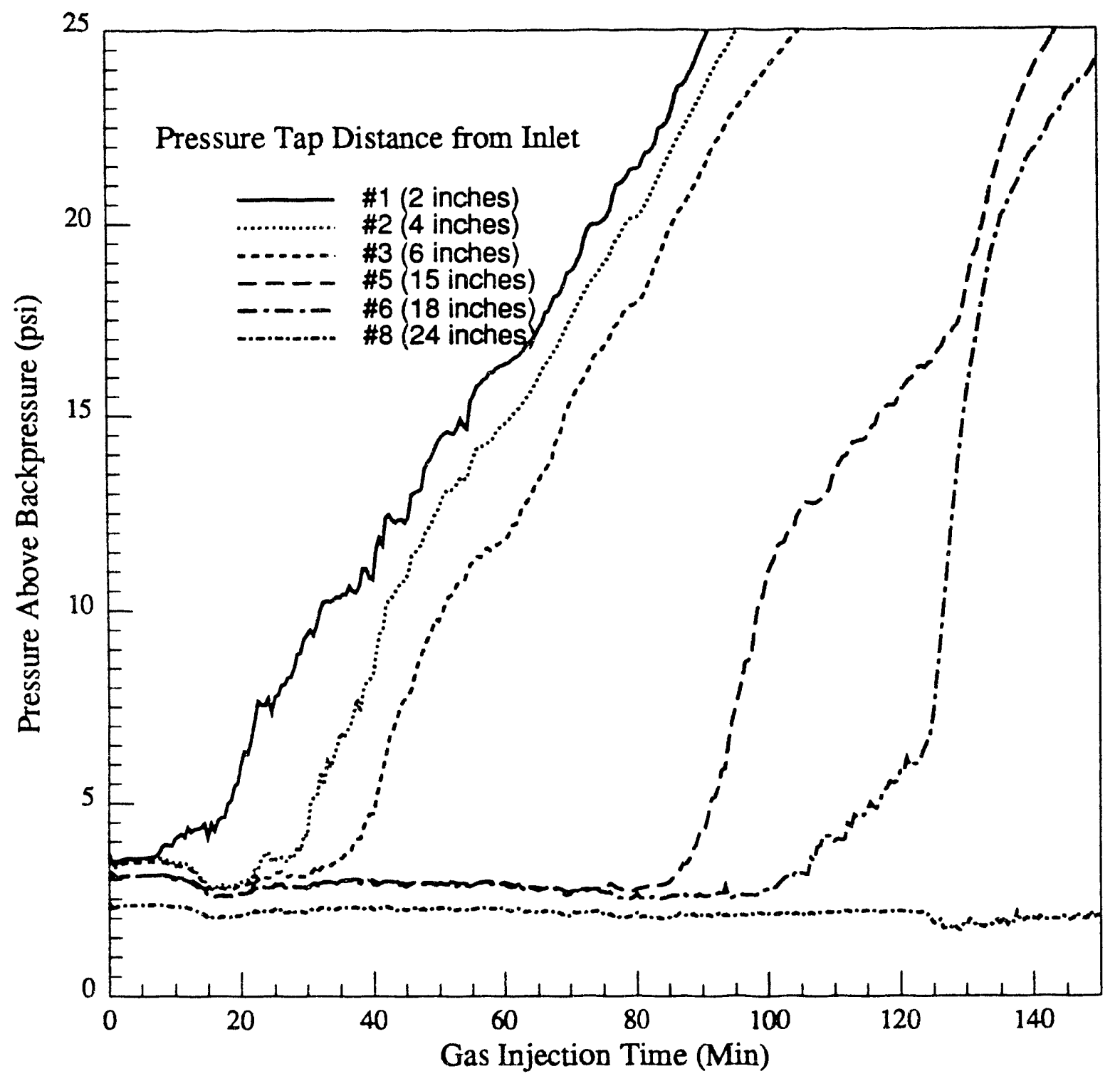

Figure 5.12: Pressure Responses for Run 34 (Gas Displacing 1\% Surfactant-Laden Solution) 
fell on top of each other to a satisfactory degree. Due to the timing during the experiments, the saturation data at 5 inches from the inlet for Run 32 (Figure 5.5) diu not show the expected sharp drop, but since no r ata was available between the first point as it started to drop to the second point, it is likely that the saturation drop was as sharp as the other saturation histories. It was also noted that for all the runs, the final liquid saturations measured close to the inlet were always higher than the final saturations for the rest of the pack. This could have been the effect of packing irregularities, or it could have been the effect of scanning so near to the inlet end.

From the pressure data of these runs (Figures 5.2, 5.4, 5.6, 5.8, 5.10, and 5.12), it can be seen that the greater the surfactant concentration, the higher the pressure drop. Also the pressure data behaved more consistently at higher surfactant concentrations. As soon as the gas(foam) front reached one pressure tap, the pressure at that point began to rise. This gives yet another way of determining where the gas front is at a given time rather than relying solely on the CT scanning and visual observations. The late horizontal lines after 60 minutes for pressure Taps 2 and 3 in Run 32 (Figure 5.6) should be ignored since the pressure drop was beyond the range of the pressure transducers during the later times.

\subsection{Surfactant Adsorption}

To measure the adsorptions of surfactant in the sandpack, a second series of experiments was run. The sandpack was initially saturated with distilled water. Then a surfactant solution of a fixed concentration was injected at a constant rate. The effluent was collected at the outlet and analyzed for surfactant concentration. These experiments were made at surfartant concentrations of $0.01 \%, 0.1 \%$, and $1 \%$, as show'n in Figures 5.13, 5.14 and 5.15. The surfactant concentration at the outlet for each run was plotted versus volume injected. The pore volume for this sandpack was $230 \mathrm{cc}$, as indicated by the vertical line in the figures.

To take into consideration the effect of surfactant adsorption, the experimental data shown in Figures 5.13, 5.14 and 5.15 were used to find the amount of adsorption by integration of the curves. Then a least squares match gave rise to the following Langmuir-type isotherm adsorption formula:

$$
C_{i s}=\frac{0.706 C_{i}}{1+10.39 C_{i}}
$$

The experimental data along with the fitting equation are shown in Figure 5.16 on a $\log -\log$ graph and Figure 5.17 on a Cartesian graph. 


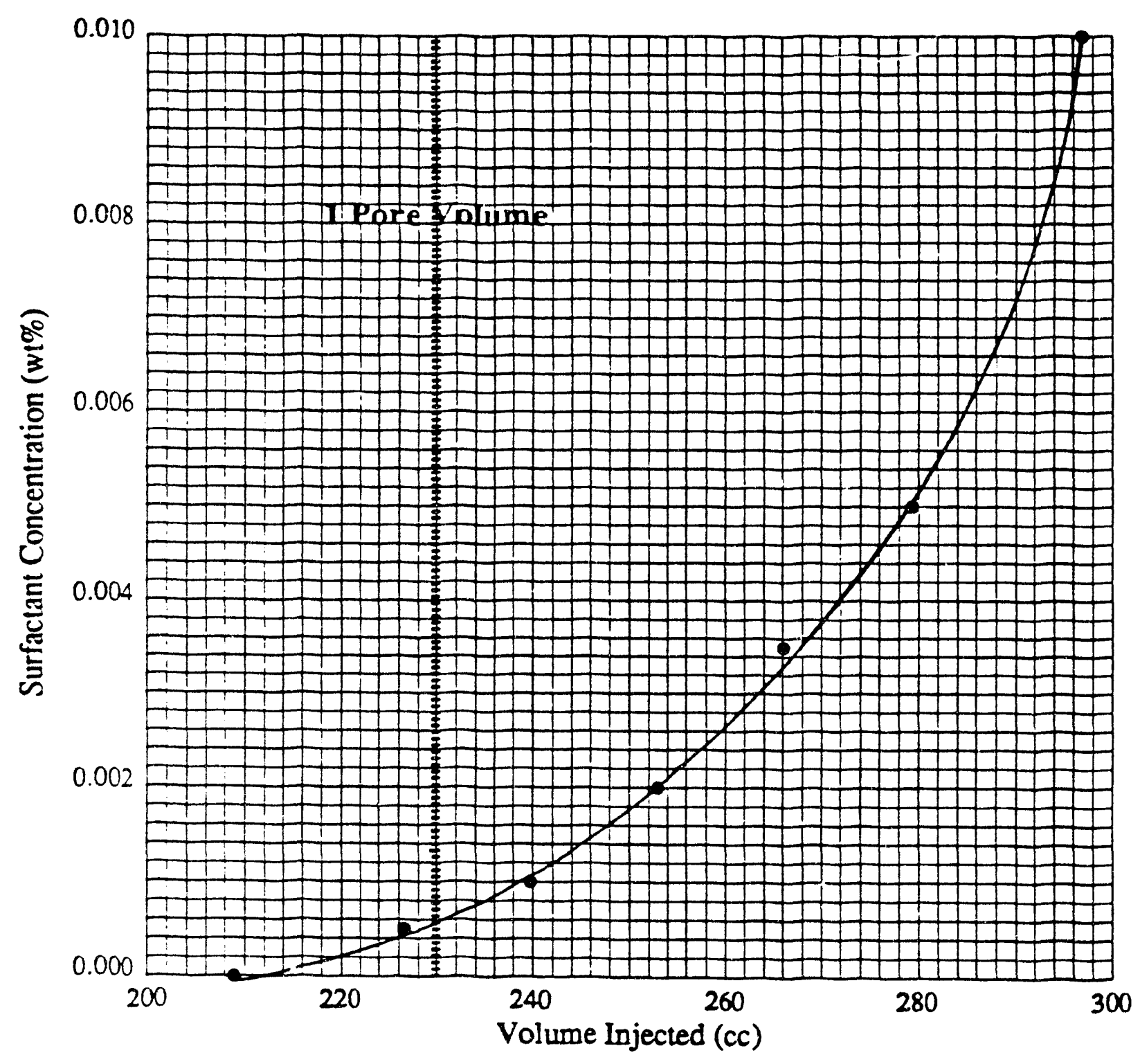

Figure 5.13: Surfactant Adsorption Measurements (at 0.01 \%) 


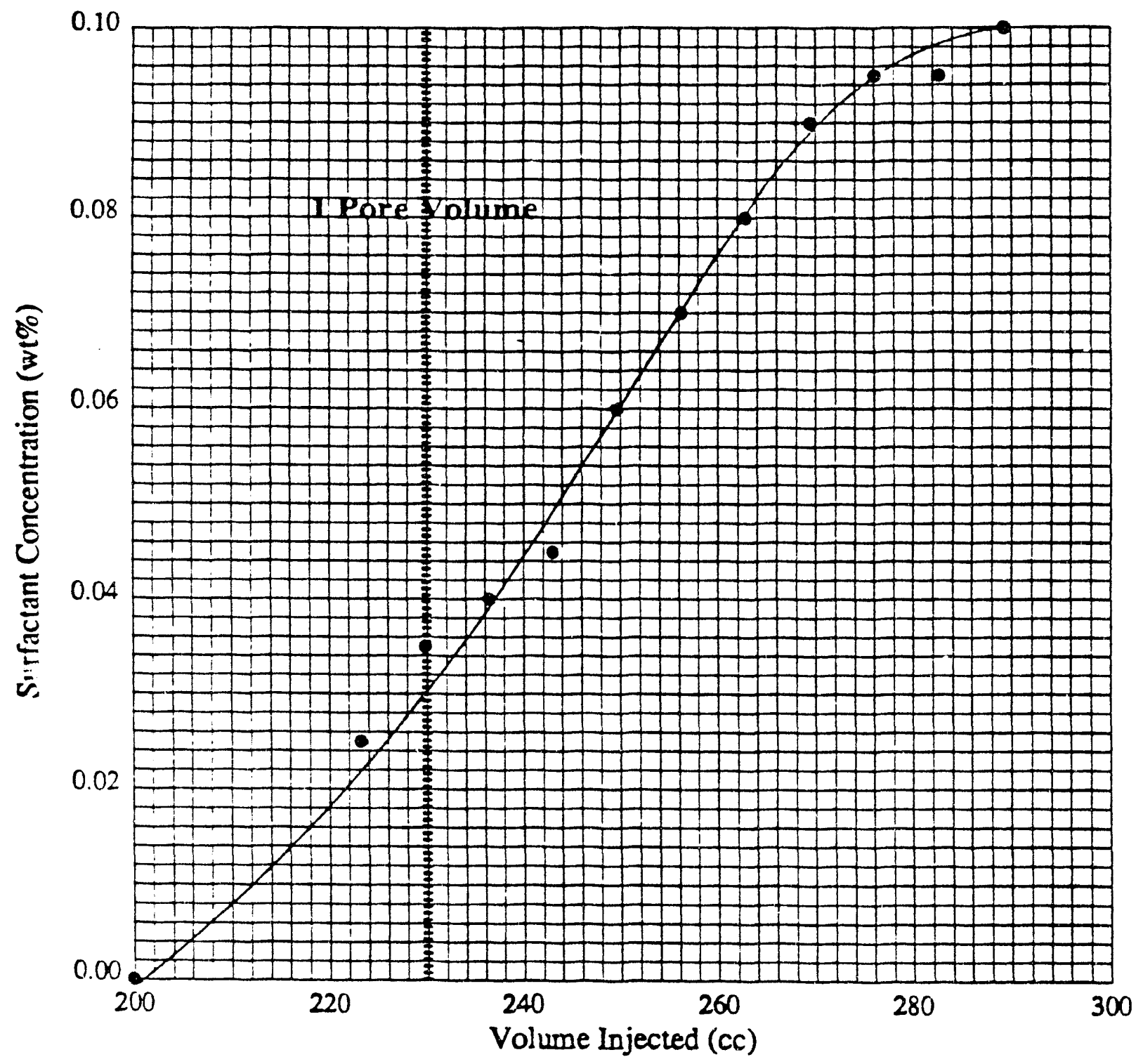

Figure 5.14: Surfactant Adsorption Measurements (at $0.1 \%$ ) 


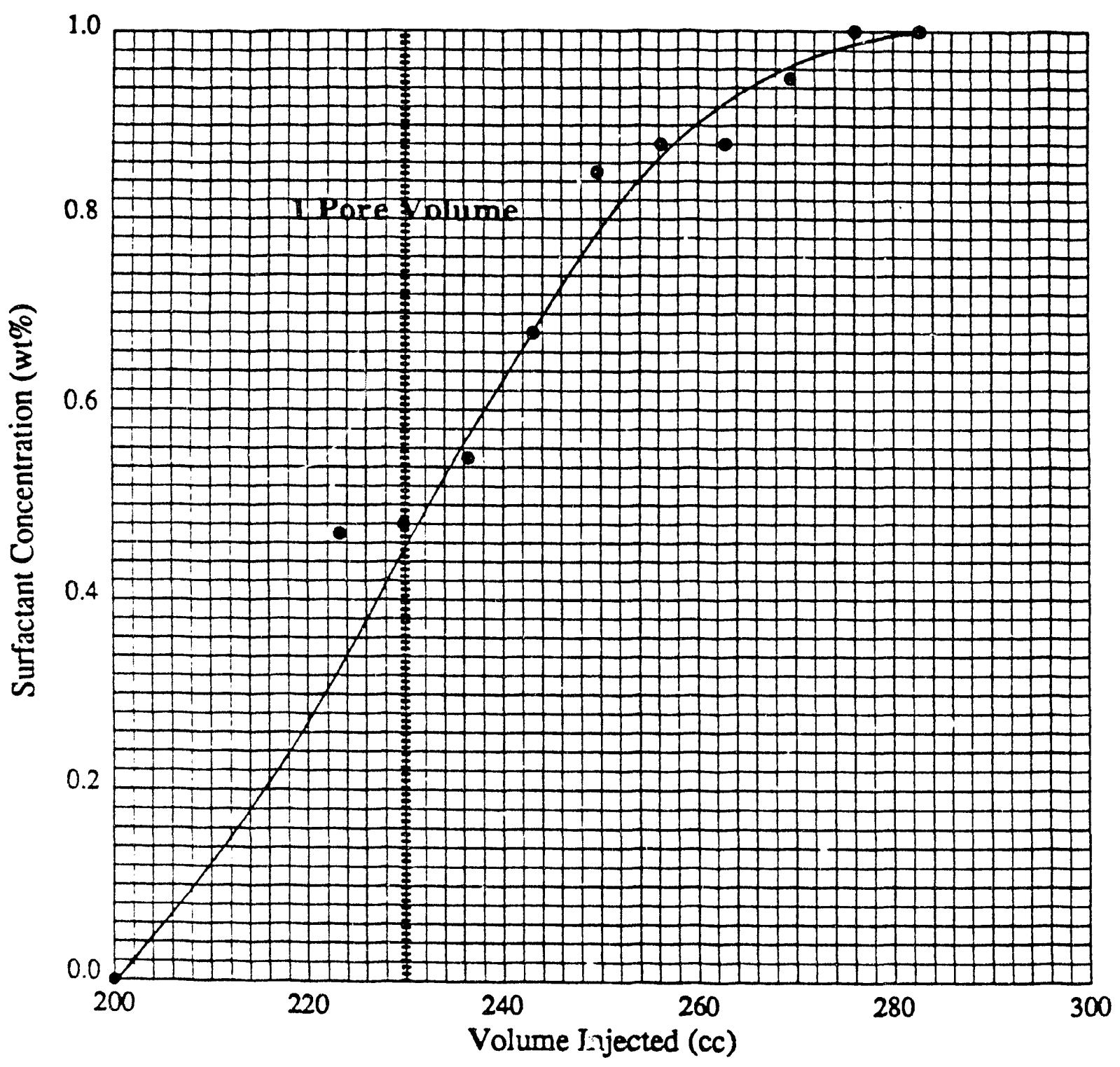

Figure 5.15: Surfactant Adsorption Measurements (at 1\%) 


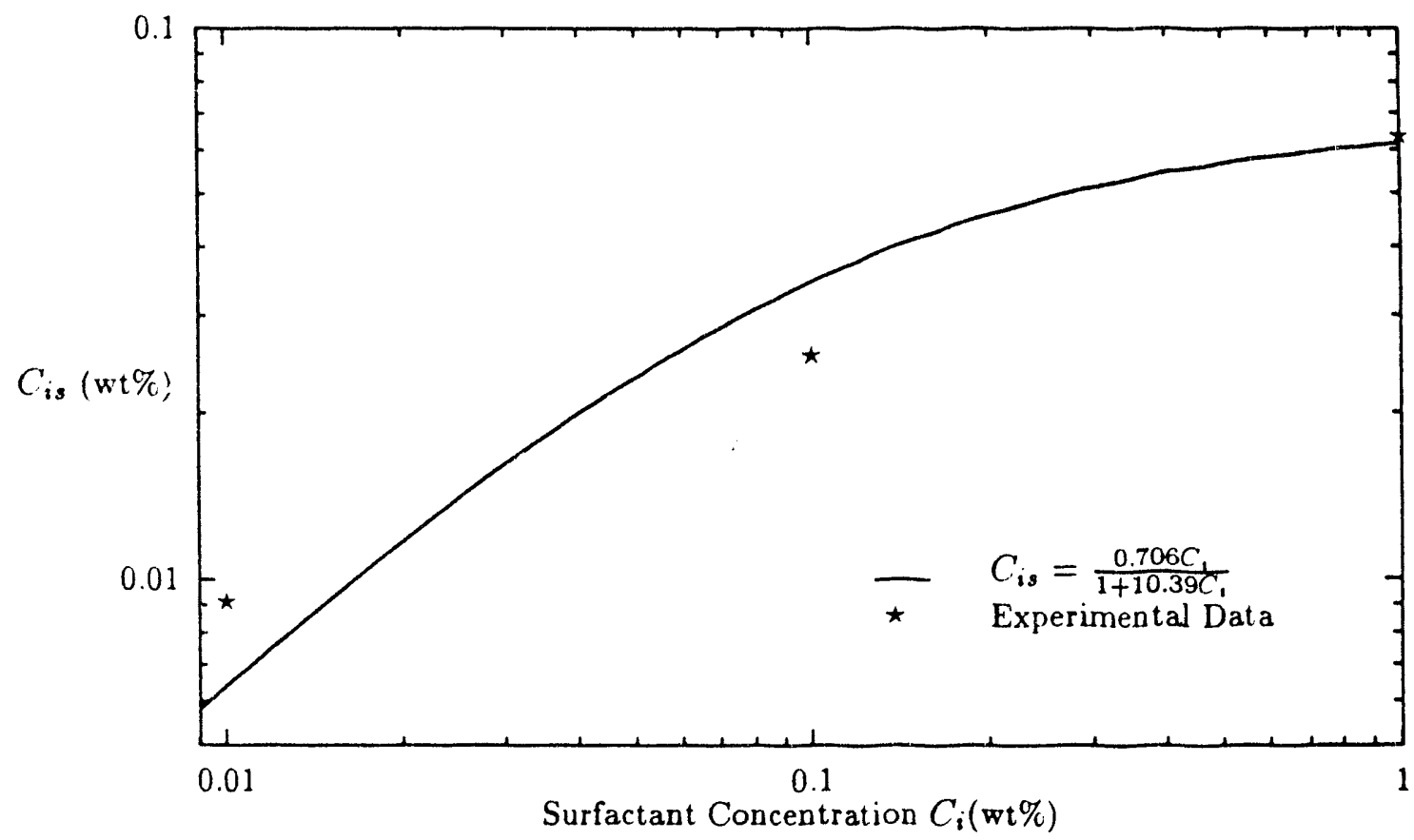

Figure 5.16: Langmuir Adsorption Curve Match (Log-Log Graph) 


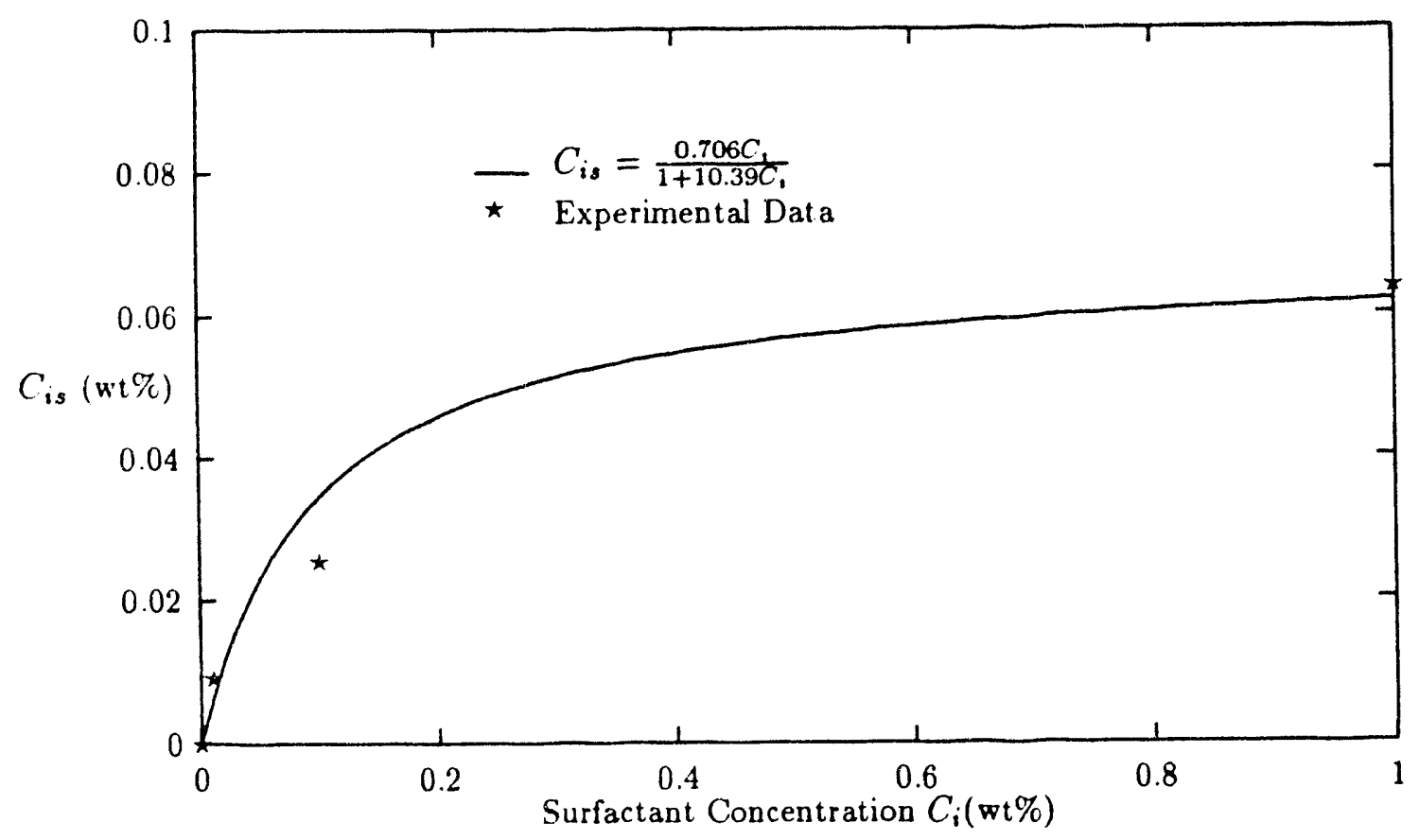

Figure 5.17: Langmuir Adsorption Match (Cartesian Graph) 


\subsection{Matching of Gas Displacement Results}

As was said in the beginning of this chapter, the main reason for doing these experiments was to find the fractional flow curves for each different surfactant concentration. All the following work was centered around finding the parameters to define the fractional flow curves for each run.

\subsubsection{Mobility of Gas at Residual Liquid Saturation}

To match the gas displacements, the first step was to find the apparent gas viscosities for each surfactant concentration. The apparent viscosities of gas were calculated using the data obtained at the end of the runs when the flow had reached steady state. They were calculated for the gas displacement runs listed in Table 5.1, using Darcy's law. That is:

$$
\mu_{a} \equiv \frac{\mu_{g}}{k_{r g}}=\frac{A k}{q} \frac{\Delta P}{L}
$$

where $\mu_{a}$ is the apparent viscosity of gas, $\mu_{g}$ is the viscosity of gas, $k_{r g}$ is the relative permeability of gas, $A$ is the cross-sectional area of the sandpack and is equal to $5.067 \mathrm{~cm}^{2}$ throughout this study, $k$ is the permeability, $L$ is the length of the sandpack, and $\Delta P$ is the pressure drop across the sandpack at steady state.

With all the other data available from Table 5.1, substitution of $\Delta P$ in Eq. 5.2 would give rise to the apparent viscosity. The apparent viscosities of gas displacement runs are shown in Table 5.2. Other parameters in this table will be discussed next.

\begin{tabular}{|c|c|c|c|c|c|}
\hline $\begin{array}{c}\text { Run } \\
\text { No. }\end{array}$ & $\begin{array}{c}\text { Surfactant } \\
\text { Concentration } \\
(\mathrm{wt} \%)\end{array}$ & $\begin{array}{c}\text { Apparent } \\
\text { Viscosity } \\
(\mathrm{cp})\end{array}$ & $\begin{array}{c}\text { Final } \\
S_{g}\end{array}$ & $\begin{array}{c}\text { Constant } \\
b\end{array}$ & $\begin{array}{c}t_{B T} \\
(\mathrm{PV})\end{array}$ \\
\hline 21 & 0.0 & 0.018 & 0.430 & 6.518 & 0.25 \\
\hline 31 & 0.001 & 0.05 & 0.599 & 11.43 & 0.45 \\
\hline 32 & 0.01 & 5.70 & 0.698 & 23.66 & 0.60 \\
\hline 35 & 0.1 & 34.2 & 0.801 & 32.89 & 0.72 \\
\hline 34 & 1.0 & 68.4 & 0.885 & 108.6 & 0.85 \\
\hline
\end{tabular}

Table 5.2: Matching Data of Gas Displacements 


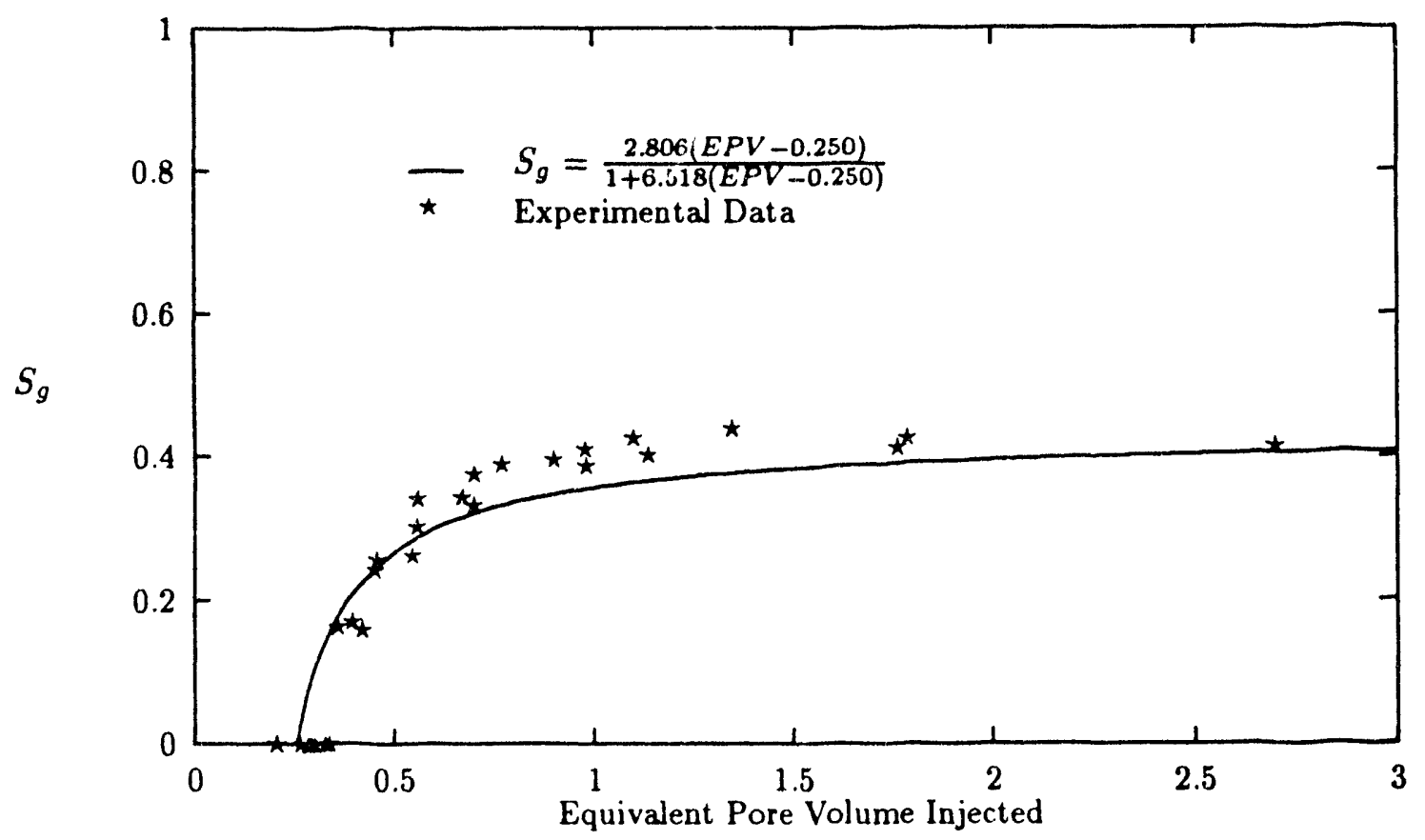

Figure 5.18: Saturation History Match for Run 21 (0.0\% Surfactant Concentration)

\subsubsection{Relative Permeabilities of Gas and Surfactant-Laden Vater}

A simpie formula was sought to smooth the saturation histories. Since the saturation histories were treated as a function of the equivalent pore volumes injected, they could also be thought of as saturation at the outlet. So, after some trial and error, the saturation histories for gas displacements, expressed as a function of the gas saturation versus EPV injected, were matched satisfactorily with a very simple, Langmuir type, formula:

$$
S_{g}=\frac{a\left(W_{i}-t_{B T}\right)}{1+b\left(W_{i}-t_{B T}\right)}
$$

where $a$ and $b$ are constants, $W_{i}$ is the pore volumes of gas injected, and $t_{B T}$ is the breakthrough time in pore volumes. The saturation histories shown in Figures 5.1, 5.3, 5.5, 5.9, and 5.11 were replotted as gas saturation curves in Figures 5.18, 5.19, 5.20, 5.21, and 5.22.

These graphs also show the curves ured to fit the data, and the equation constants using Equation 5.3. These constants are listed in Table 5.2. 


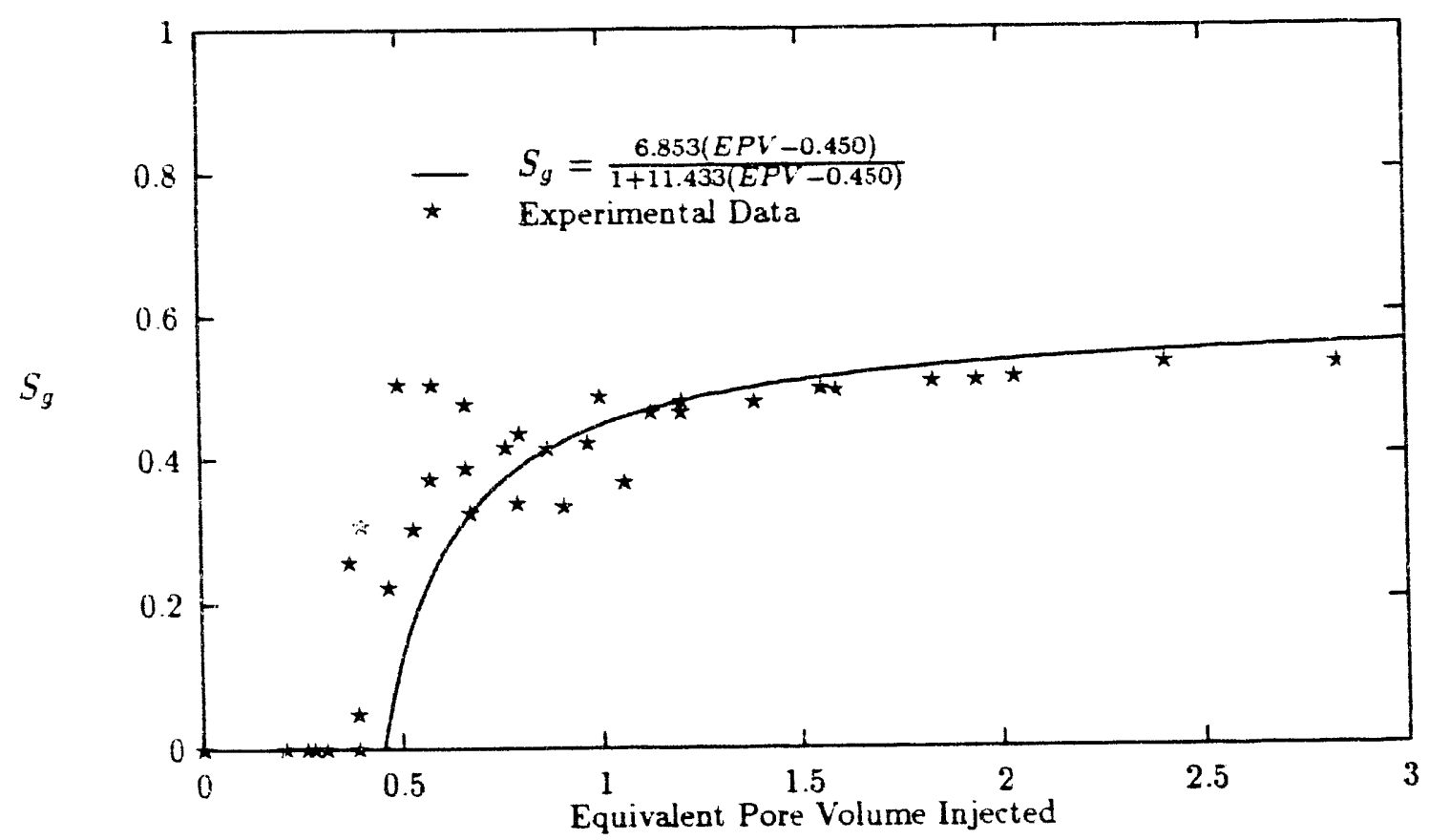

Figure 5.19: Saturation History Match for Run 31 (0.001\% Surfactant Concentration) 


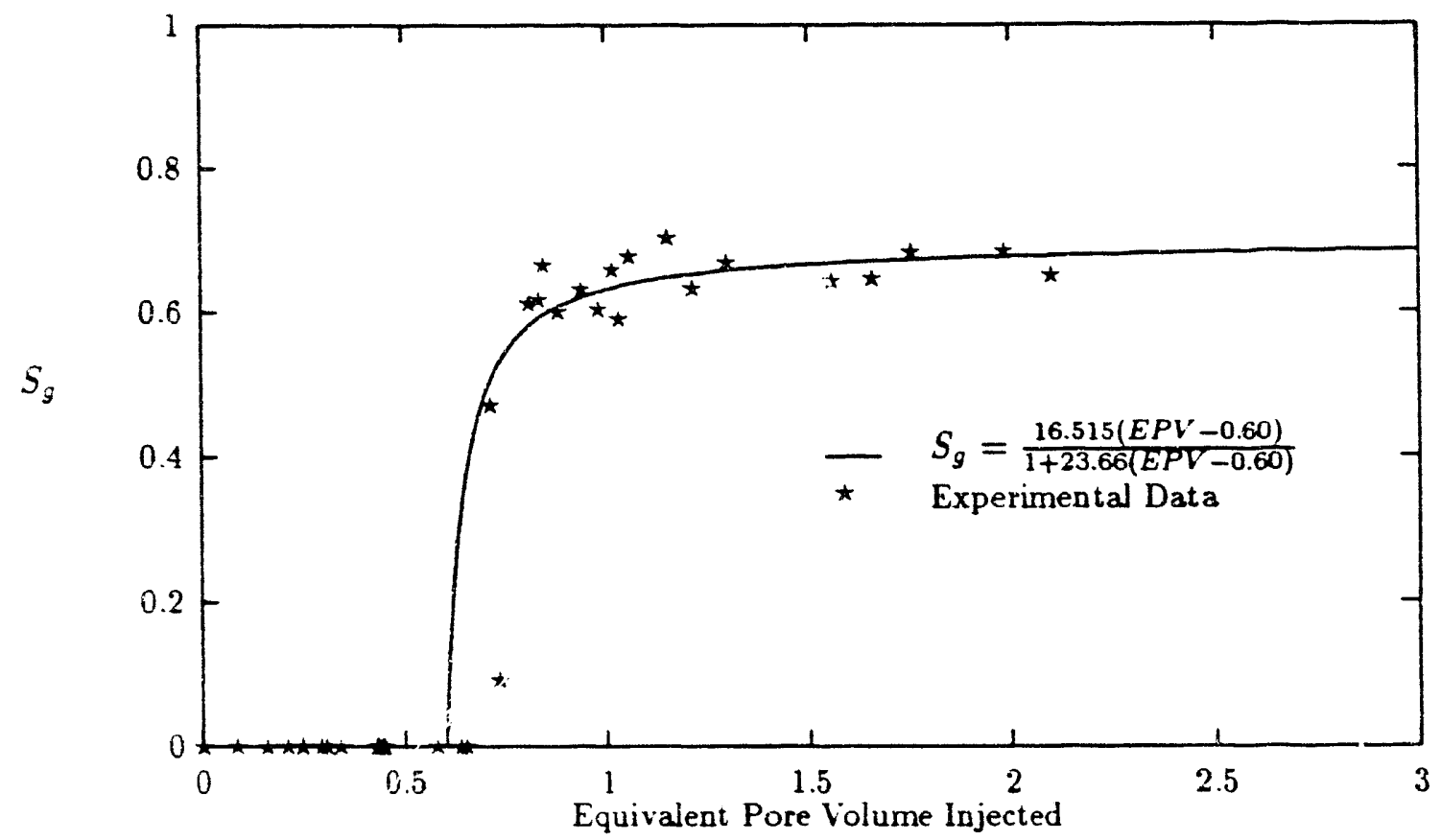

Figure 5.20: Saturation History Match for Run 32 (0.01\% Surfactant Concentration) 


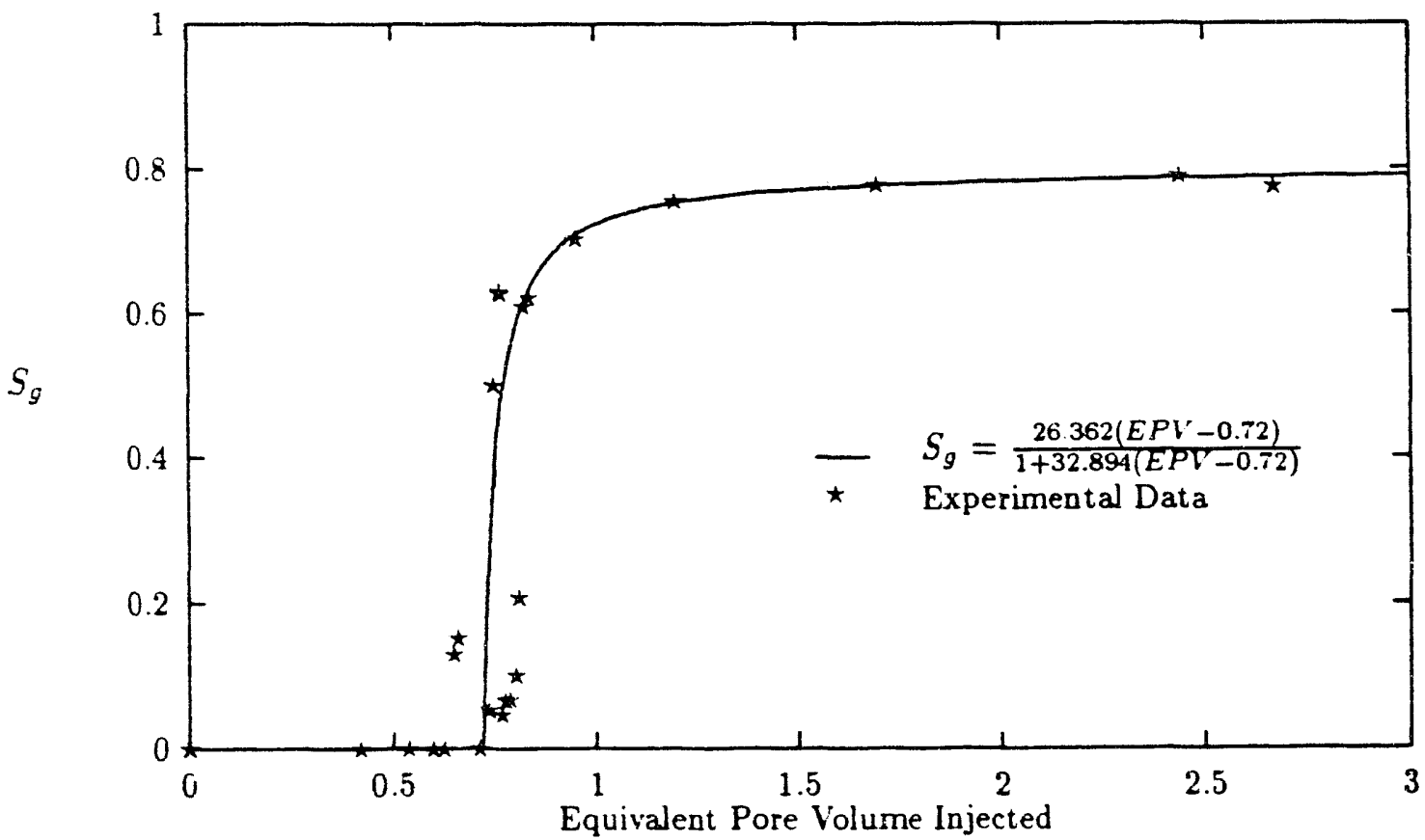

Figure 5.21: Saturation History Match for Run 35 (0.1\% Surfactant Concentration) 


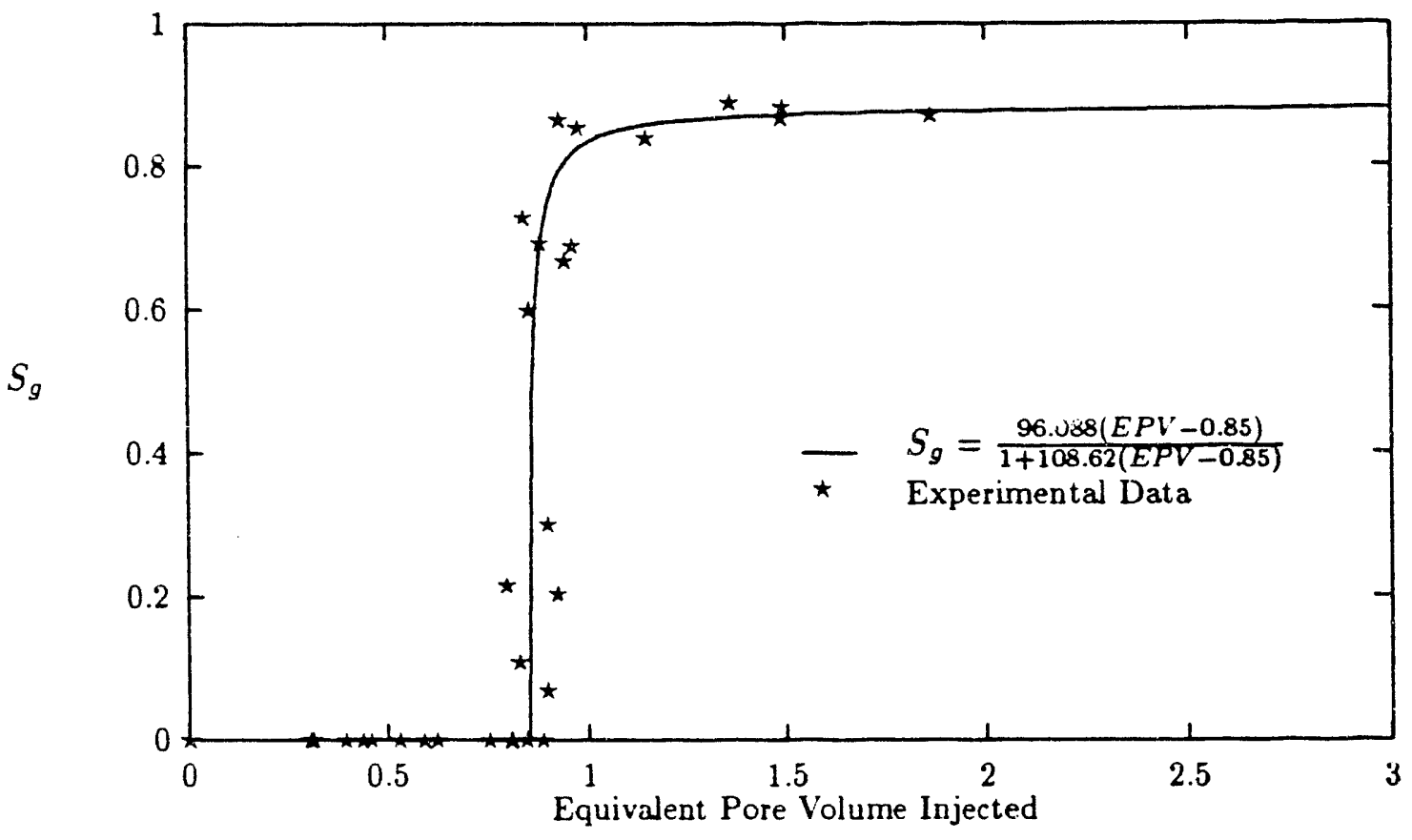

Figure 5.22: Saturation History Match for Run 34 (1.0\% Surfactant Concentration) 
Care was taken to preserve the material balance in the matching process. That is the reason that some of the matches were not the best in the sense of a least squares fit. The breakthrough times were estimated using the pressure profiles and the recovery data.

Let us try to see if the relative permeabiuities can be generalized into the power law type equations (Equations 4.1 and 4.2 ) rather than the type that have been used here. We will take Run 21, the gas-water system as an example. Given a value of relative permeability index for water, $n_{w}$, the relative permeability of gas, $k_{r g}$ could be calculated from the ratio $k_{r w} / k_{r g}$. Many values of $n_{w}$ were tried and some of the results are shown in Figure 5.23 for $n_{w}$ equal to $1.0,1.5,1.93$, and 2.5 . It can be seen that it is very difficult to obtain a straight line from these curves. Another example is given in Figure 5.24 for Run 31 in which gas was injected to displace surfactant-laden solution of $0.001 \%$ concentration. Three values of $n_{w}$ are shown at 1.0, 1.5 and 1.85. Again, a straight line was hard to obtain. This means that a relative permeability index for gas can not be obtained as a power function, even for the case of pure gas-water displacement. The other runs gave even worse curves.

Notice also, that the nearest to a straight line in Figures 5.23 and 5.24 would give slopes less than 1.0 on the $\log -\log$ scales, which means $n_{g}$ would be less than 1.0. Again this is contrary to the behavior usually associated with Equations 4.1 and 4.2. Hence the conclusion is that the power law relative permeability equations do not work in ou- case. Thus it was necessary to use the experimental data in this work to obtain the fractional flow curves as will be discussed later. It turned out that Equation 5.3 was the best form found that could match all the data adequately.

The final gas saturation of the displacements is reached when $W_{i}$ approaches infinity. From Equation 5.3, the final gas saturation is the ratio of $a$ to $b$. These values are also listed in Table 5.2.

\subsubsection{Interpolating Equations for Gas Displacements}

The matching coefficients in Table 5.2 could be used to define each separate frastional flow curve at a fixed surfactant concentration, but since a continuous change of surfactant concentration was the goal, they were then converted to interpolation formulas. Figure 5.25 shows the experimental data and the least squares fit of the final gas saturation as a function of surfactant concentration. In this graph, the number 0.00001 was added to the value of the concentration so that the pure water data $(0.0$ concentration) would be included in the data set for correlation. Using this graphing technique, a straight line was obtained. The final gas saturation depends on the surfactant concentration in the following manner:

$$
S_{g}=0.8854+0.0922 \log \left(C_{\mathbf{i}}+0.00001\right)
$$




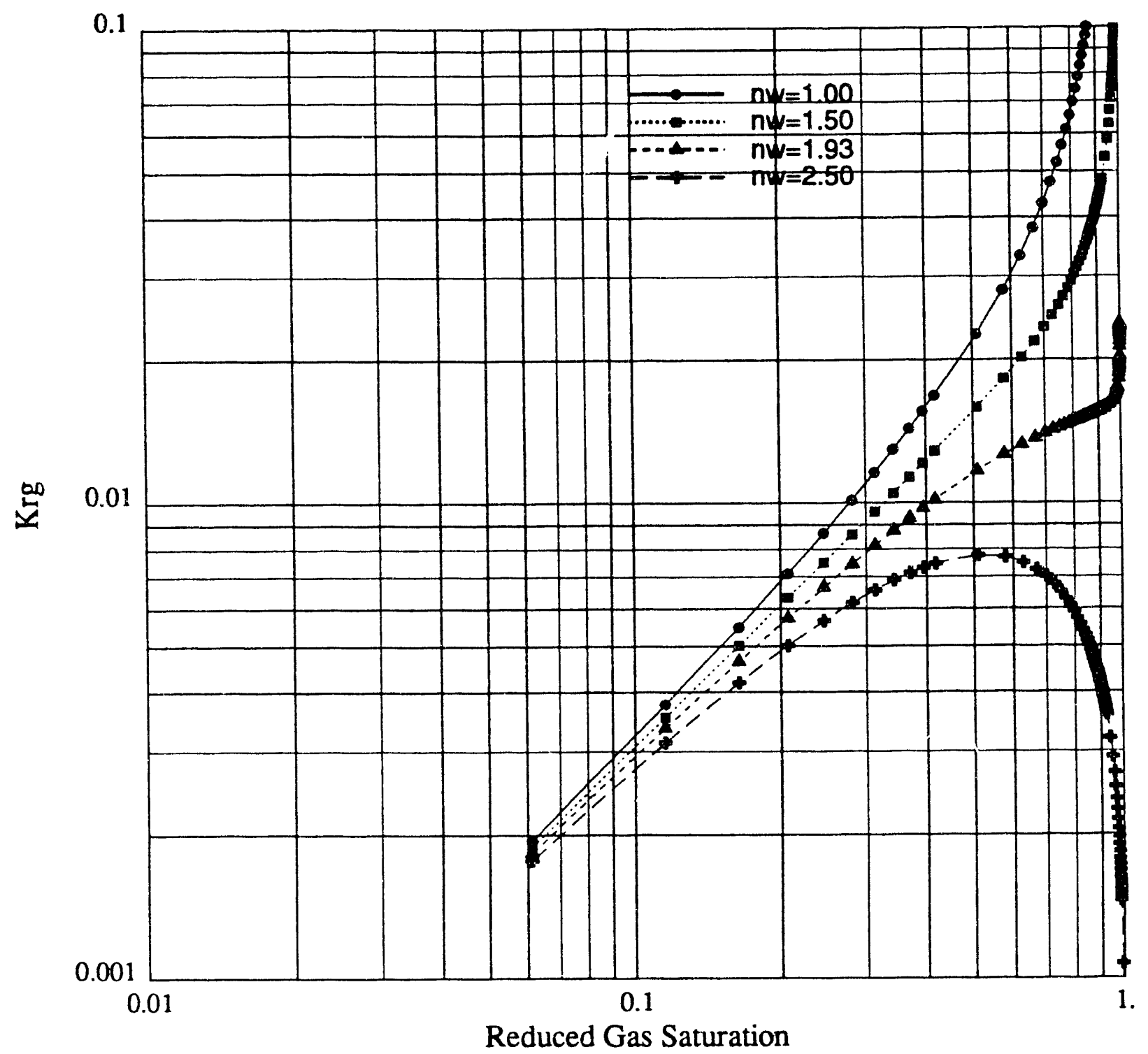

Figure 5.23: Calculated Gas Relative Permeabilities from Run 21 Data 


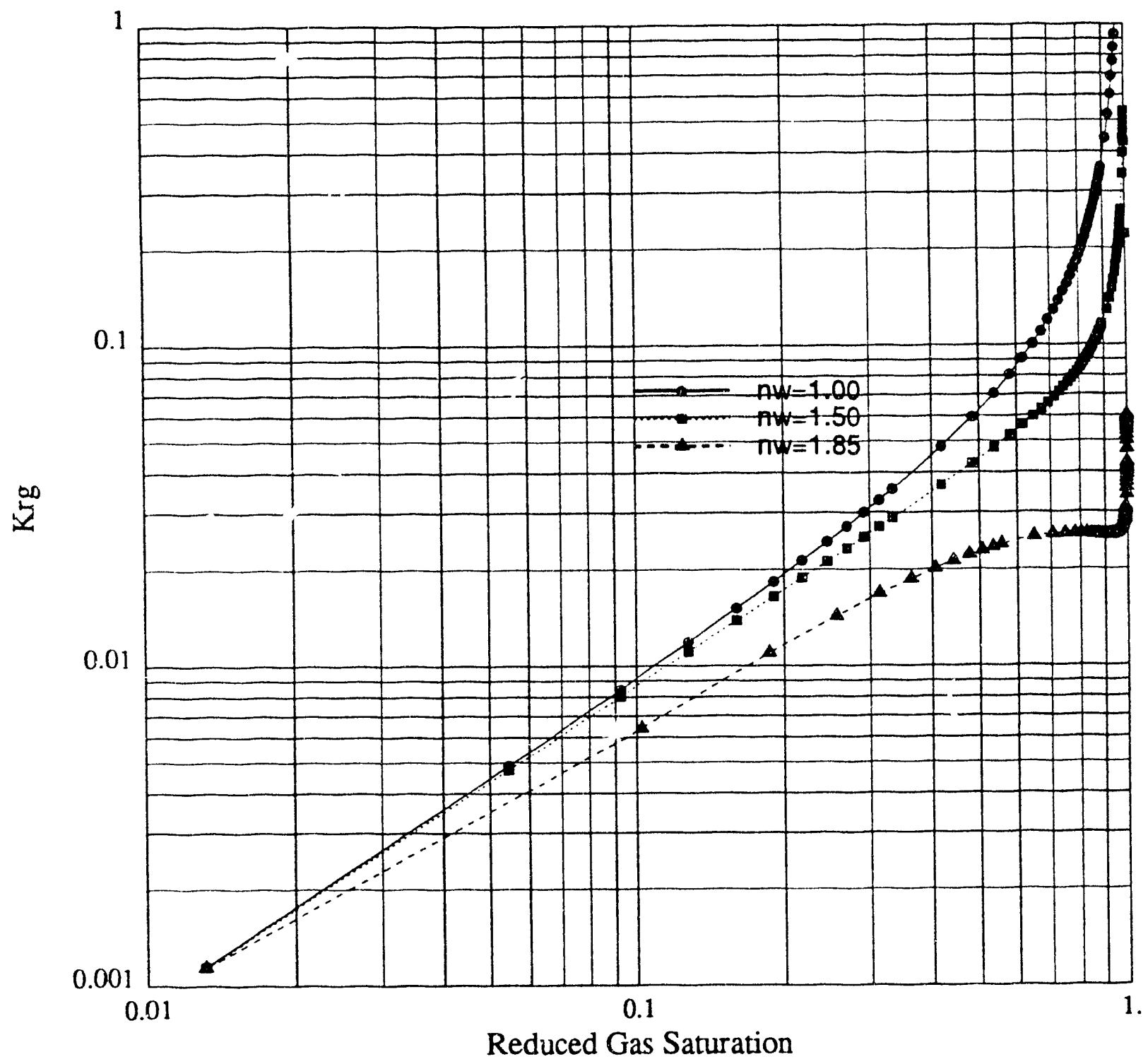

Figure 5.24: Gas Relative Permeability of Run 31 from Experimental Data 


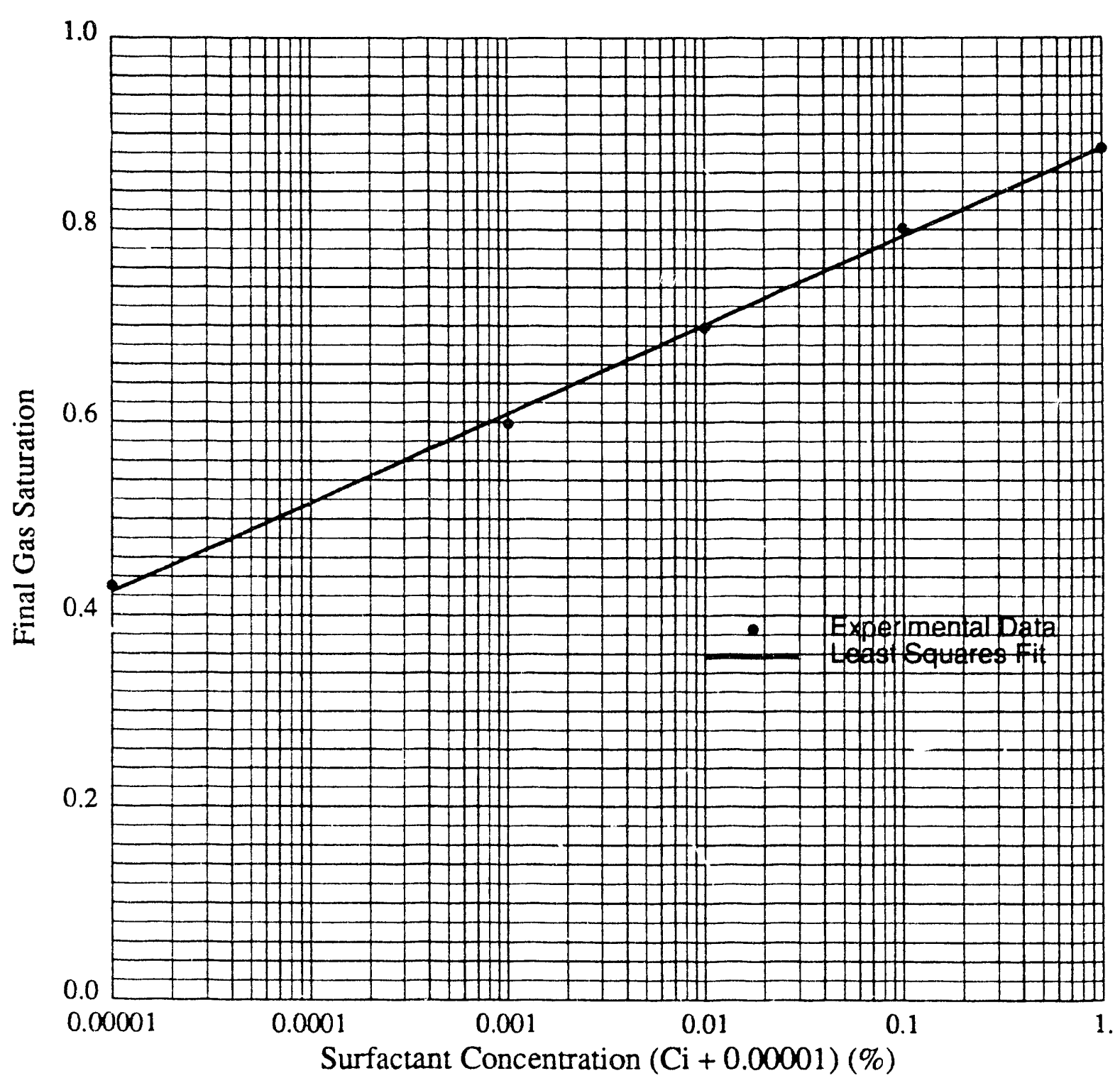

Figure 5.25: Relationship between Surfactant Concentration and Gas Saturation 
The dependence of breakthrough time (in pore volumes) on the surfactant concentration also exhibits an almost exact straight line when the constant 0.00001 is added to the concentration, as shown in Figure 5.26:

$$
t_{B T}=0.8406+0.1212 \log \left(C_{i}+0.00001\right)
$$

In matching the saturation histories using Equation 5.3, another condition, the material balance, was implied. That means that to define a matching equation, the three constants (final gas saturation, $t_{B T}$, and $b$ ) also have to obey the material balance. Since two straight line relationships were found for both the final gas saturation and the breakthrough time as a function of surfactant concentration, the relationship between $b$ and the surfactant concentration could be calculated from material balance. In so doing, the data for the coefficient $b$ and the calculated data are shown in Figure 5.27.

It can be seen from this figure that the calculated values for $b$ do not follow the experimental data closely, especially at the concentration values of 0.001 and 0.1 , although the other two constants (final gas saturation and breakthrough time) were quite close to the straight lines in Figures 5.25 and 5.26. This indicates ths, $b$ does not play as important a role as the other two constants in defining the matching curve of saturation histories. It appears that the worst case for these interpolations was for $\mathrm{R}$ un 31 from these figures. So some further work was done to see the effect of these interpolations on the fractional flow results and will be shown later in this section.

The apparent viscosity of gas also varies with surfactant concentration. Since it was not used in any of the matchings and would not be used for future interpolations, no attempt was made to obtain a mathematical equation from the data. Instead, a curve was drawn simply to show the trend of change, as shown in Figure 5.28.

Having obtained these relationships that define a unique saturation history given a surfactant concentration, the fractional flow curve can be fousd at any concentration, as described next. Equation 5.3 can be used to define the saturation change as a function of the pore volumes injected $\left(W_{i}\right)$ at the outlet $\left(X_{d}=1\right)$. With a little manipulation of Equation 5.3, the saturation at any time and distance can be expressed as follows:

$$
S_{g}\left(W_{i}, X_{d}\right)=\frac{a W_{i}-a t_{B T} X_{d}}{b W_{i}-\left(b t_{B T}-1\right) X_{d}}
$$

The saturation at the outlet is simply (at $\left.X_{d}=1\right)$ :

$$
S_{g}\left(W_{i}\right)=\frac{a W_{i}-a t_{B T}}{b W_{i}-\left(b t_{B T}-1\right)}
$$




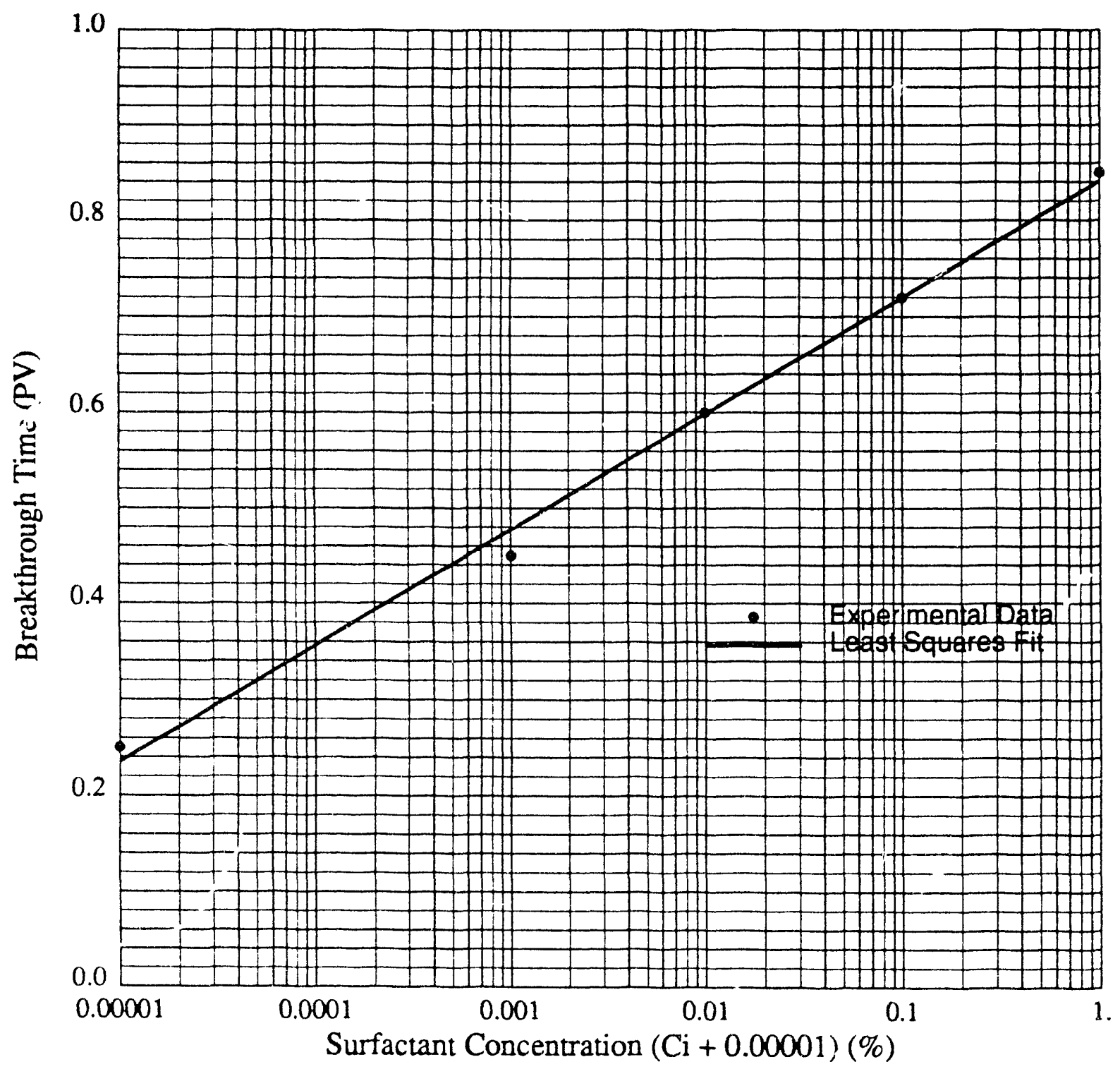

Figure 5.26: Relationship between Breakthrough Time and Surfactant Concentration 


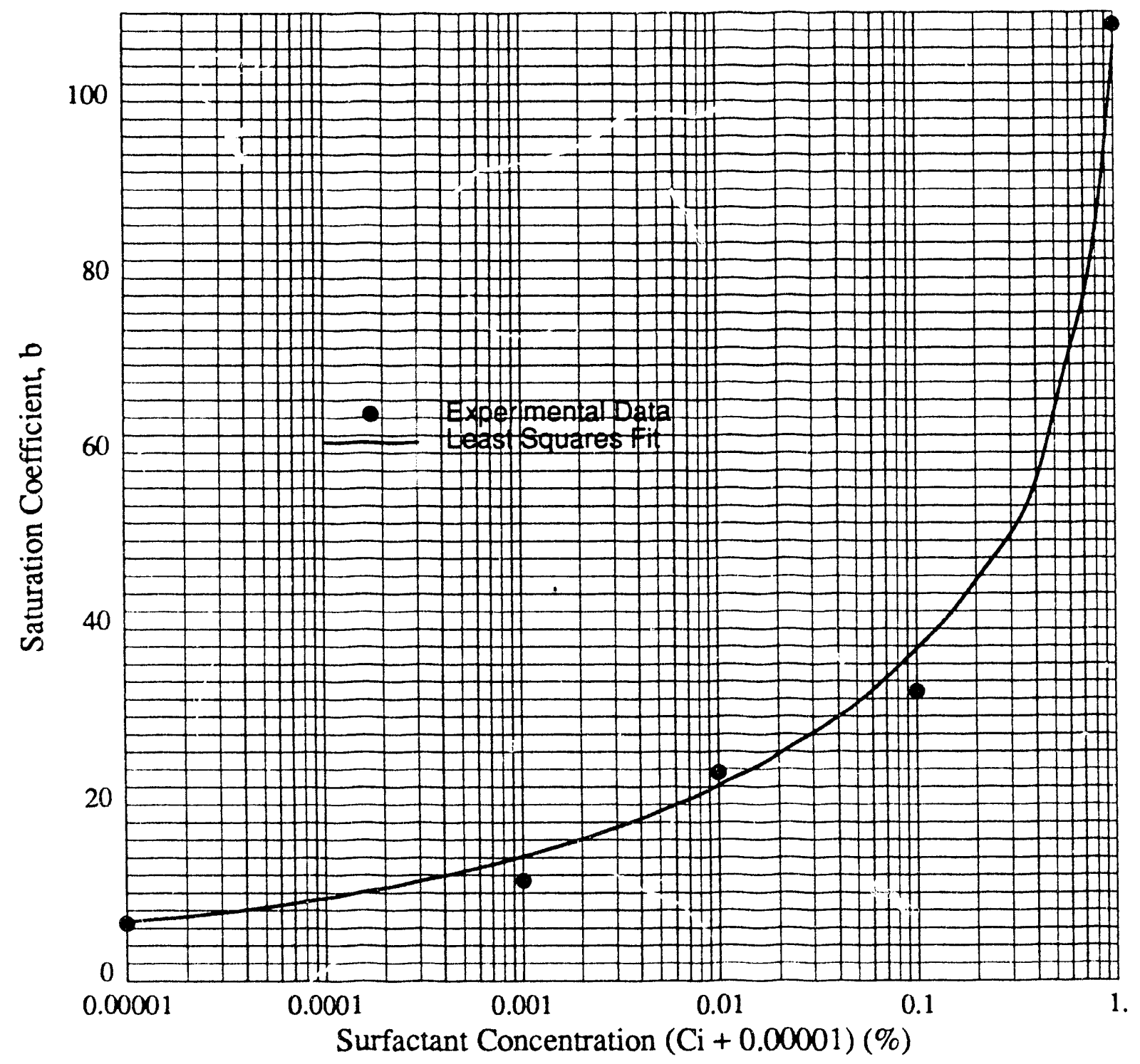

Figure 5.27: Calculated Saturation Coefficient, $b$, as a Function of Surfactant Concentration 


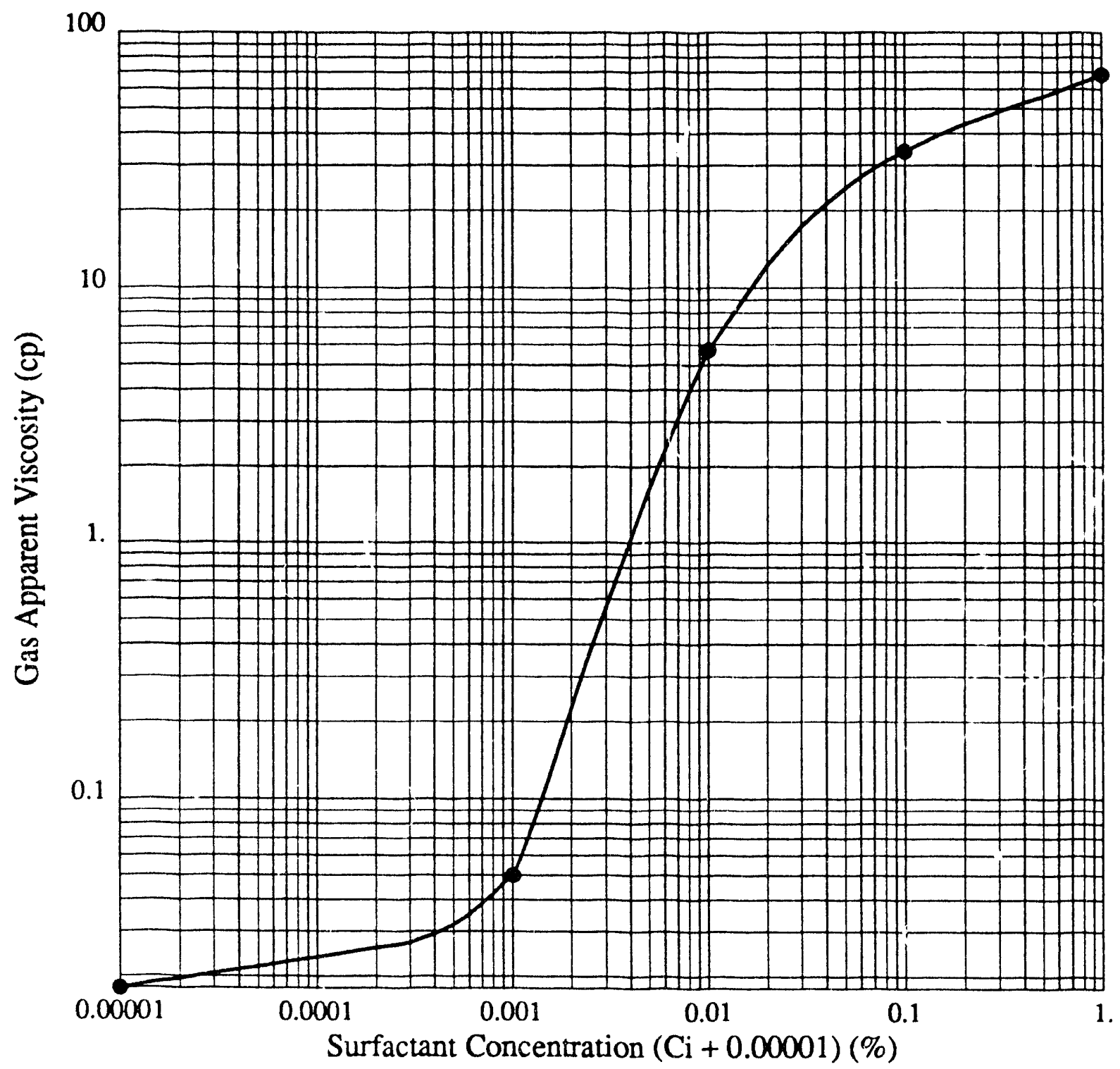

Figure 5.28: Apparent Viscosity as a Function of Surfactant Concentration 
Then $W_{i}$ can also be expressed in terms of $S_{g}$ at the outlet, from Equation 5.7:

$$
W_{i}^{\prime}=t_{B T}+\frac{S_{g}}{a-S_{g} b}
$$

The average saturation at any time $\left(W_{i}\right)$ can be calculated from the integration of the saturation in Equation 5.6 from the inlet to the outlet:

$$
\bar{S}_{g}=\int_{0}^{1} S\left(W_{i}, X_{d}\right) d X_{d}=\int_{0}^{1} \frac{a W_{i}-a t_{B T} X_{d}}{b W_{i}-\left(b t_{B T}-1\right) X_{d}} d X_{d}
$$

Let $d=a t_{B T}$ and $f=b t_{B T}-1$, the integration becomes,

$$
\overline{S_{g}}=\left(\frac{b W_{i} d}{f^{2}}-\frac{a W_{i}}{f}\right)\left[\ln \left(b-\frac{f}{W_{i}}\right)-\ln (b)\right]+\frac{d}{f}
$$

The fractional flow is then calculated from Welge's equation (Brigham, 1979) as:

$$
f_{g}=1-\frac{\bar{S}_{g}-S_{g}}{W_{i}}
$$

To verify this theoretical work, the recovery data from the experimental runs were matched using Equation 5.10. Since the initial gas saturation in all the runs was zero, the recovery factor was equal to the average gas saturation in the sandpack. So the recovery daia was matched with $\bar{S}_{g}$. The fits were generally good for all the runs as shown in Figures 5.29 through 5.33 for Runs $21,31,32,35$ and 34 . These results furiher confirm the validity of the saturation matches, the form.s of the fractional flow curves, and the constants derived from the gas displacement data.

As mentioned before, the effect of the interpolation relationships obtained before should be further investigated. Since Run 31 appeared to be the worst case, the constants obtained before and after the inierpolations were used to calculate the fractional flow curves. As can be seen fron! Figure 5.34, the effect is not large.

The fractional flow curves for all these runs are summarized in Figure 5.35. Interpolation can be used to obtain a fractional flow curve of any surfactant concentration using the matching coefficients found above. 


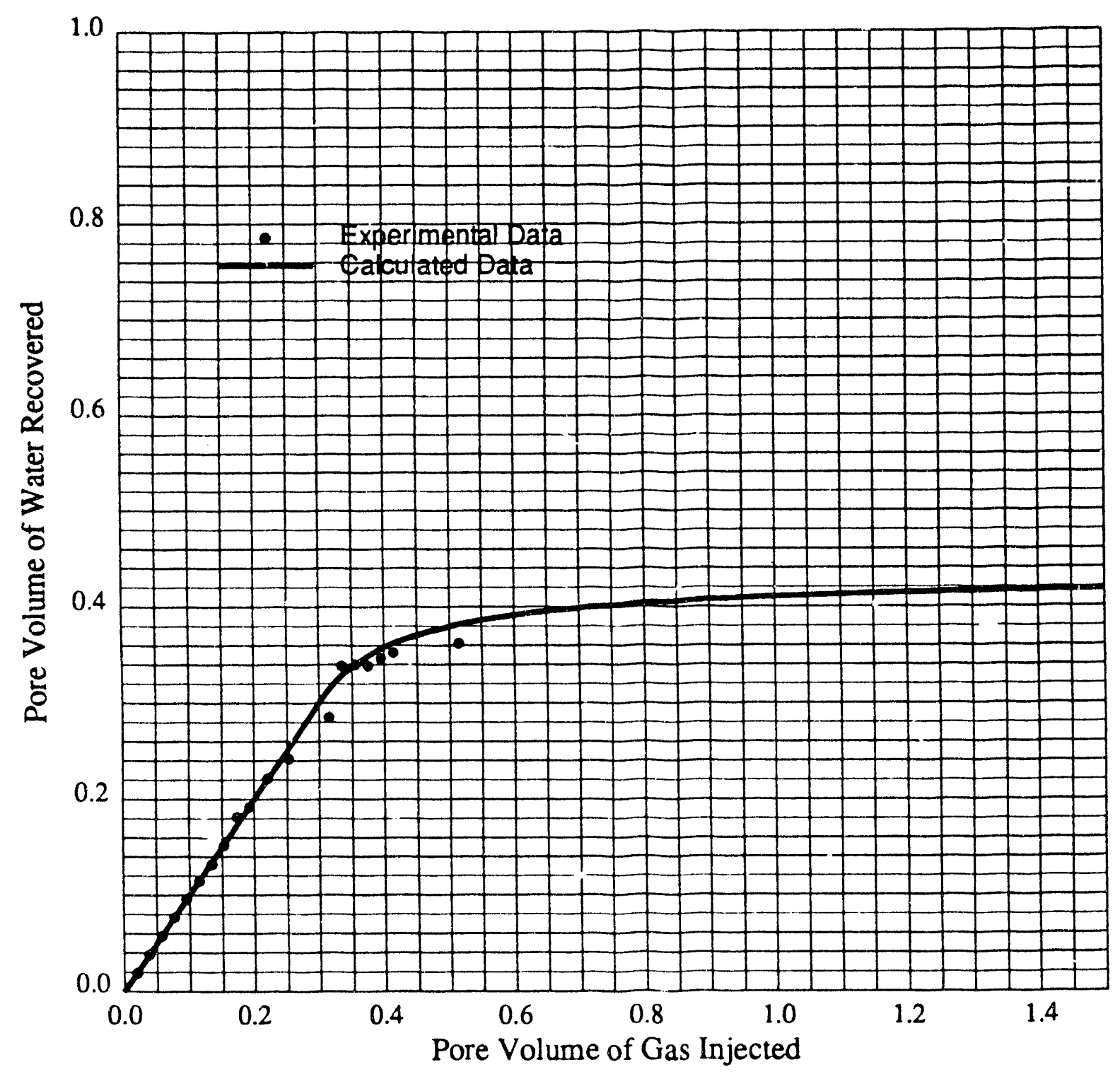

Figure 5.29: Recovery Data Match for Run 2i (C.0\% Surfactant Concentration) 


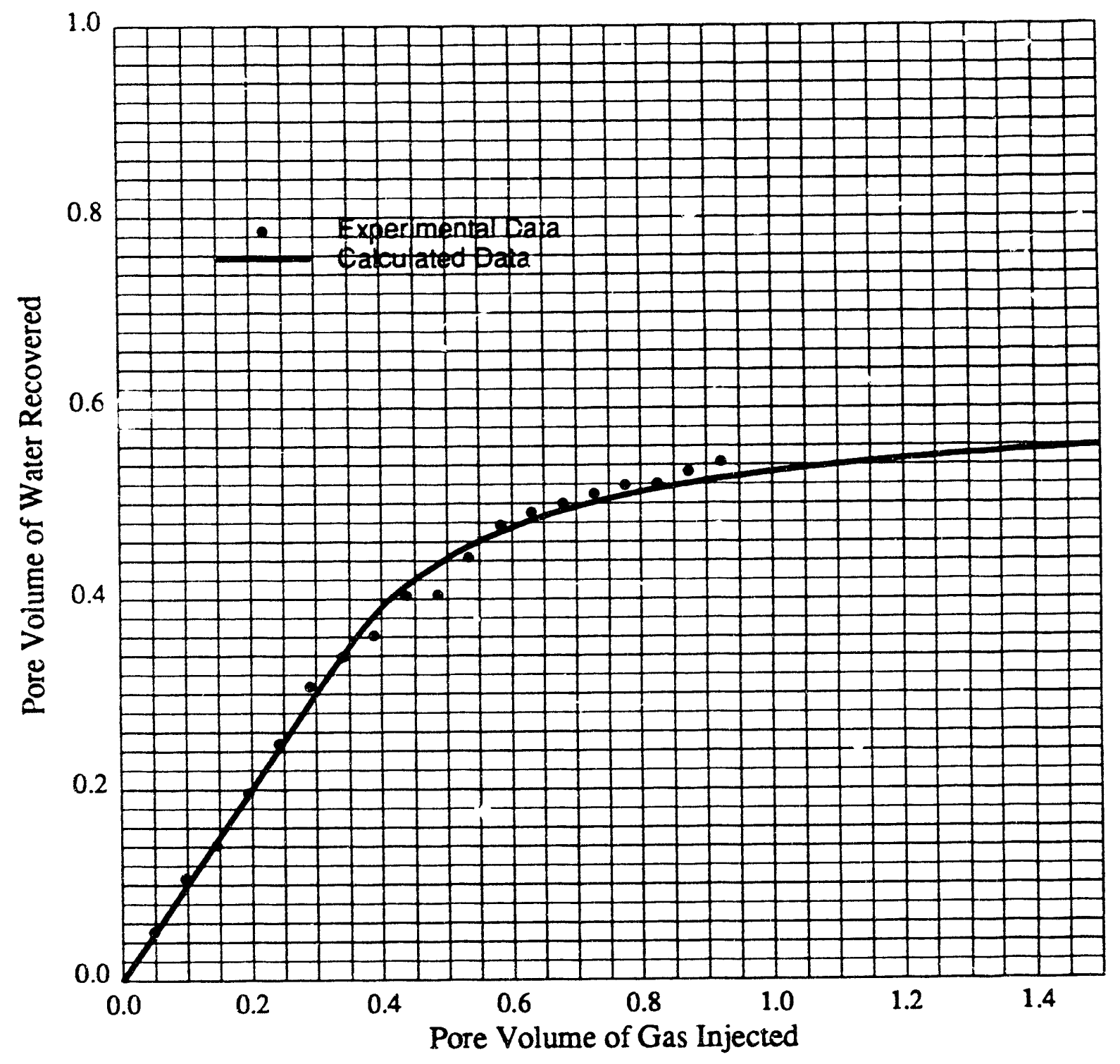

Figure 5.30: Recovery Data Match for Run 31 (0.001\% Surfactant Concentration) 


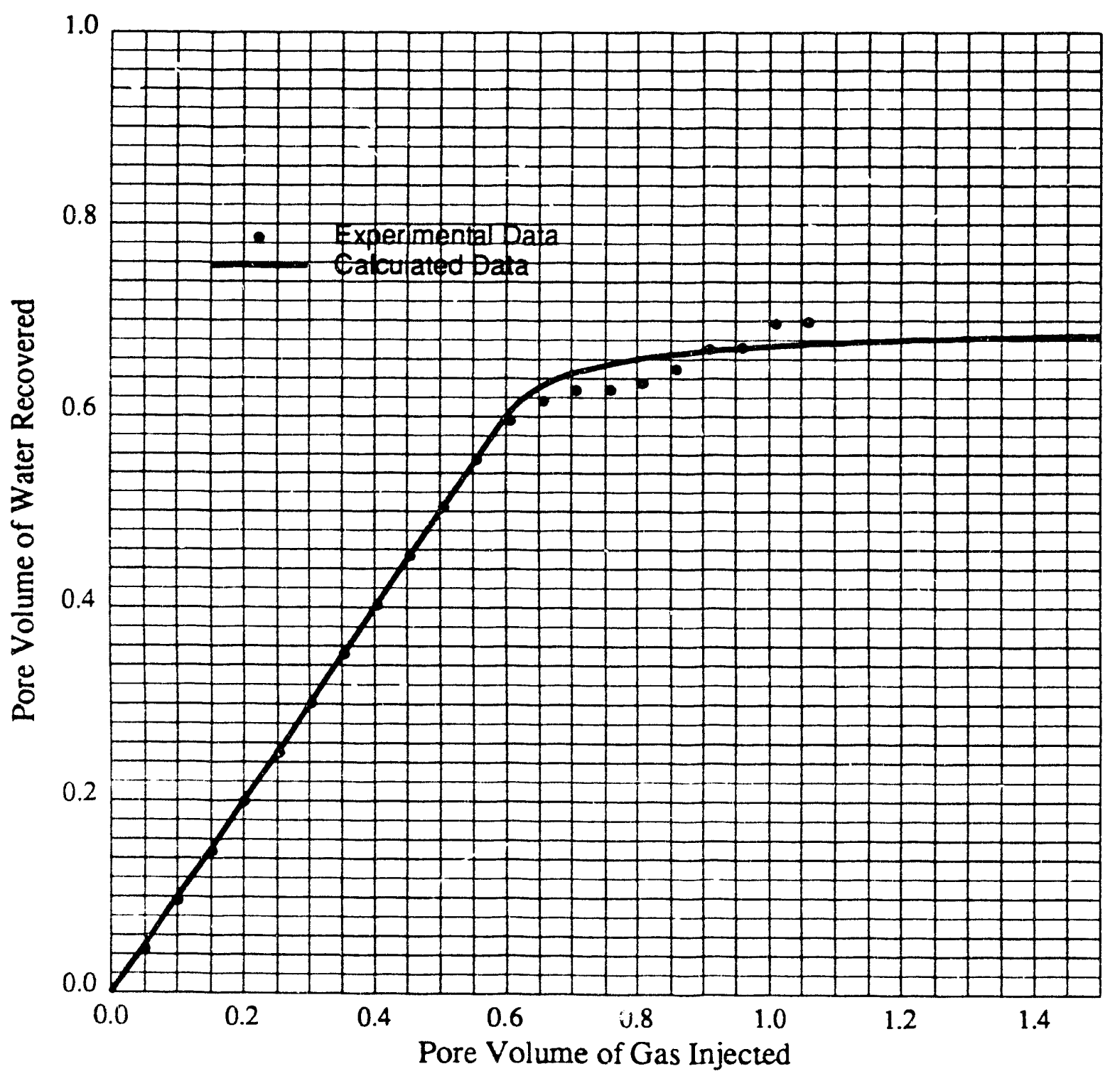

Figure 5.31: Recovery Data Match for Run 32 (0.01\% Surfactant Concentration) 


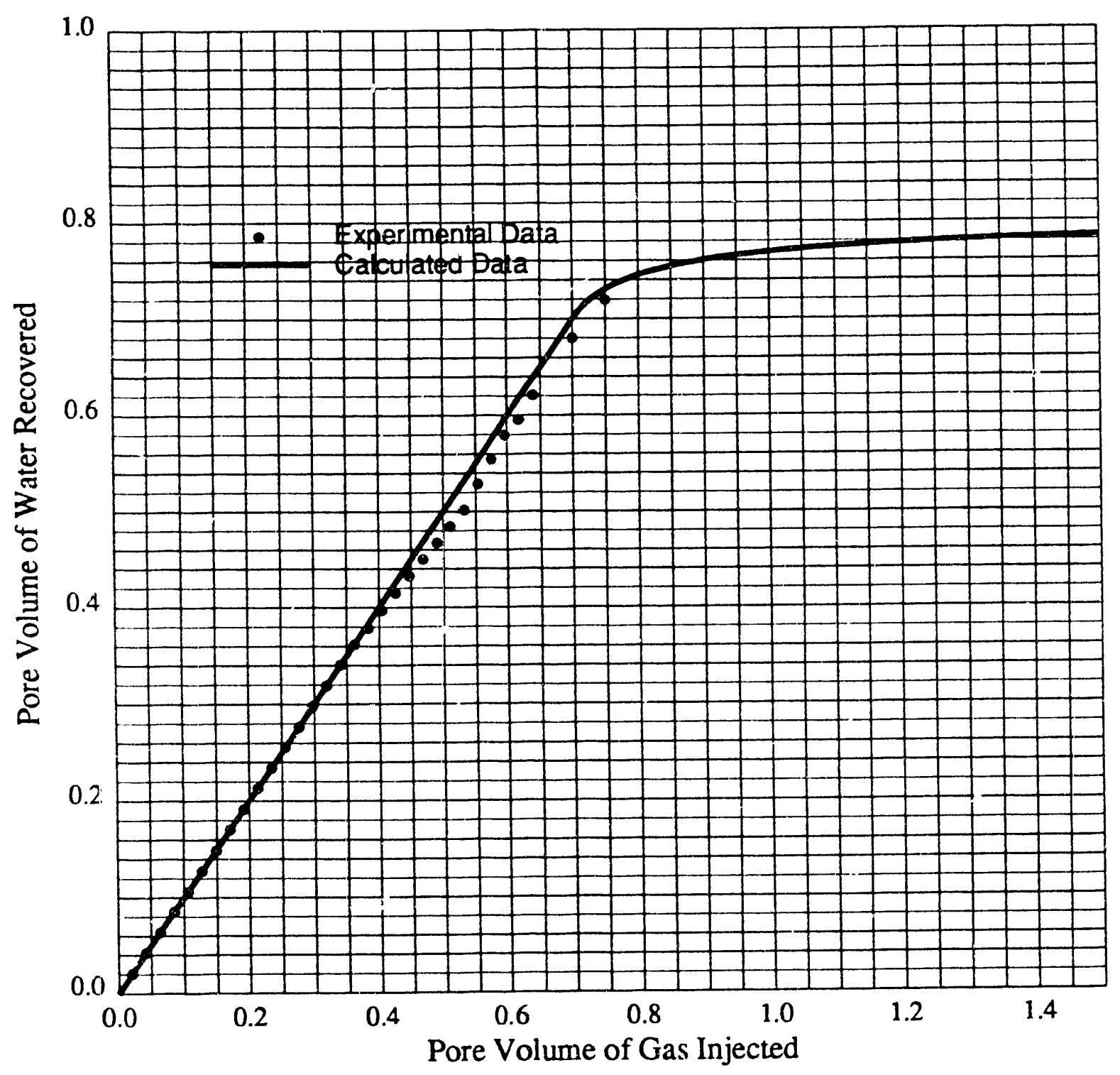

Figure 5.32: Recovery Data Match for Run 35 (0.1\% Surfactant Concentration) 


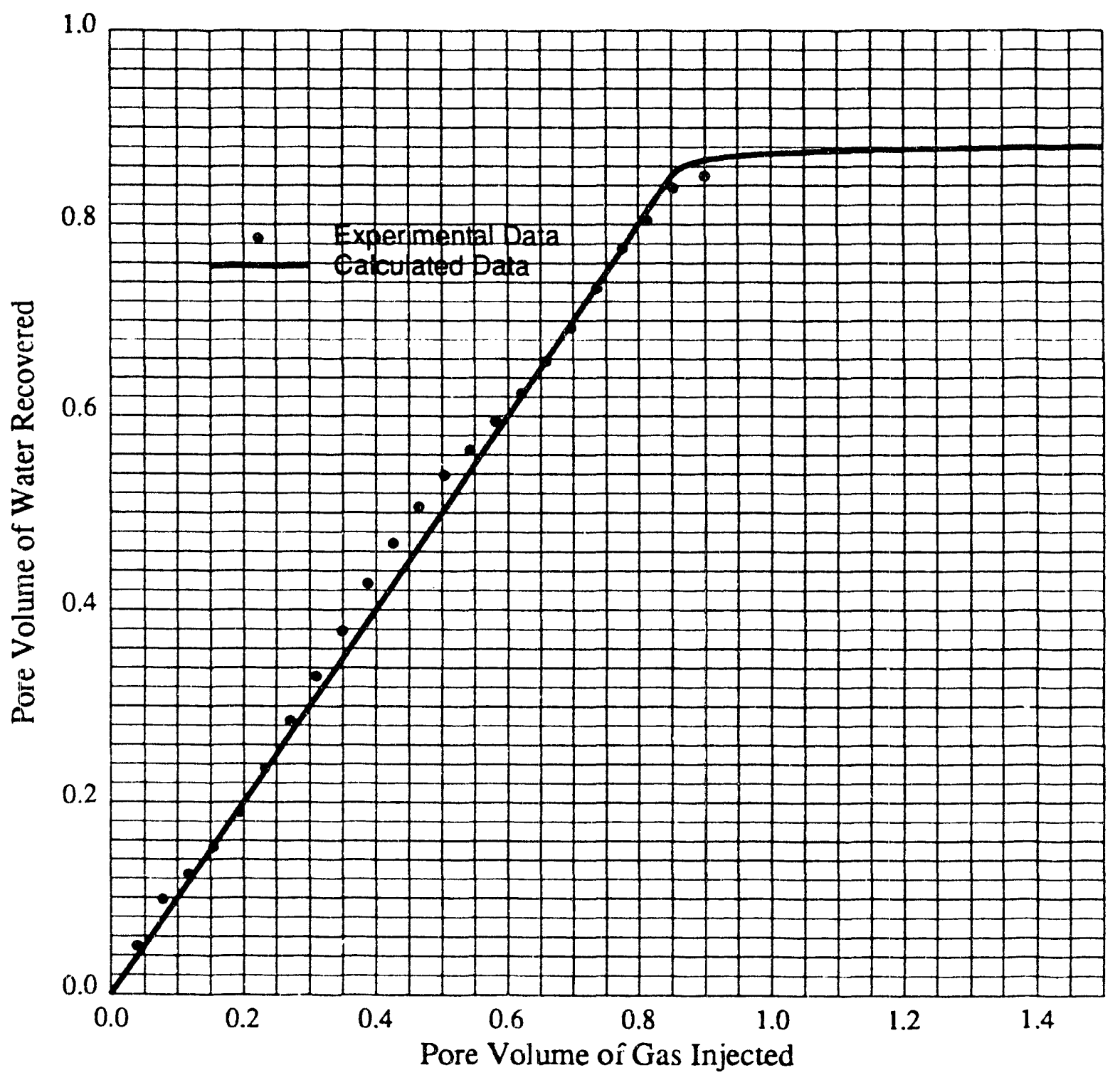

Figure 5.33: Recovery Data Match for Run 34 (1.0\% Surfactant Concentration) 


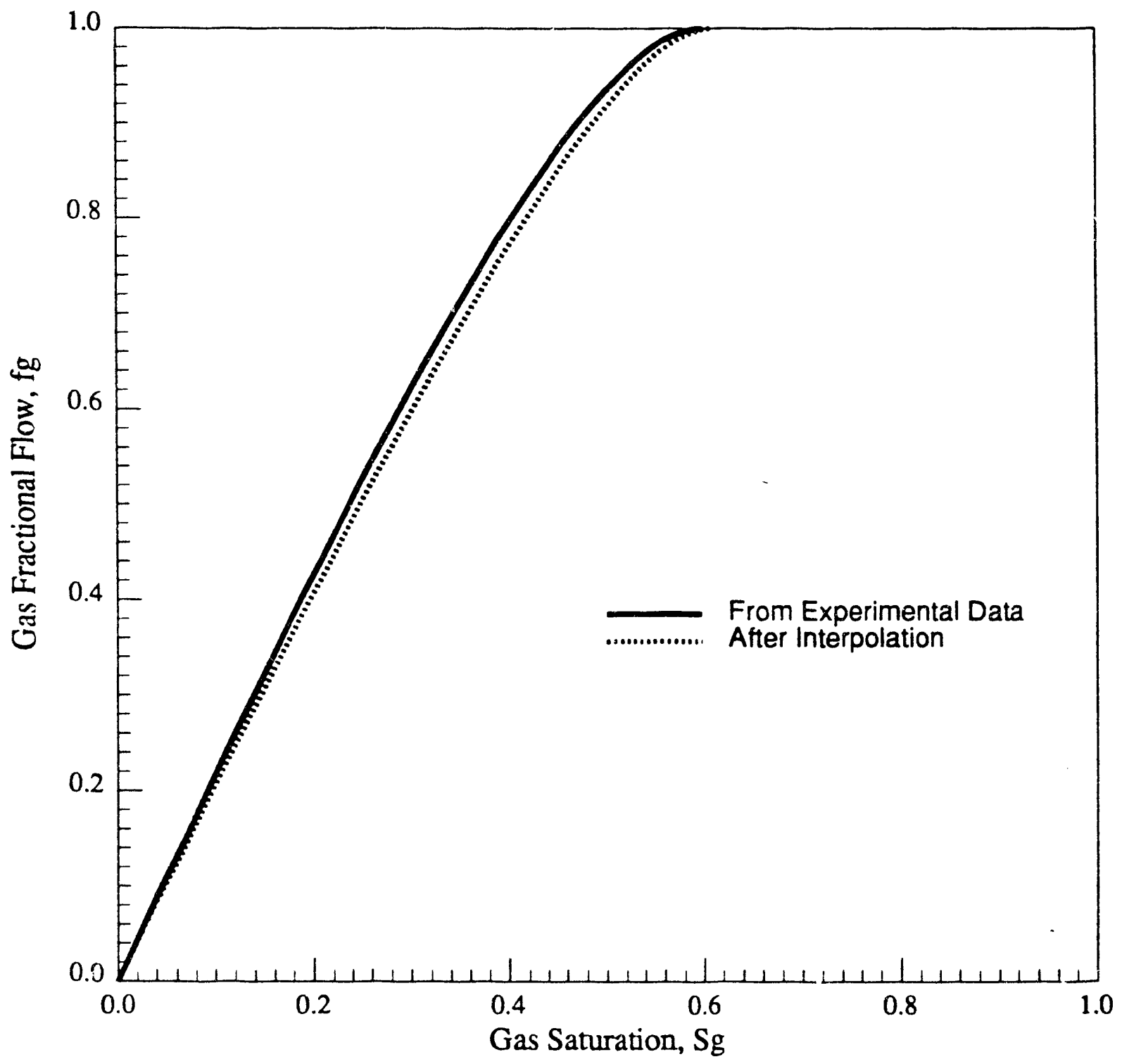

Figure 5.34: The Effect of Interpolations on Fractional Flow Curves for Run 31 


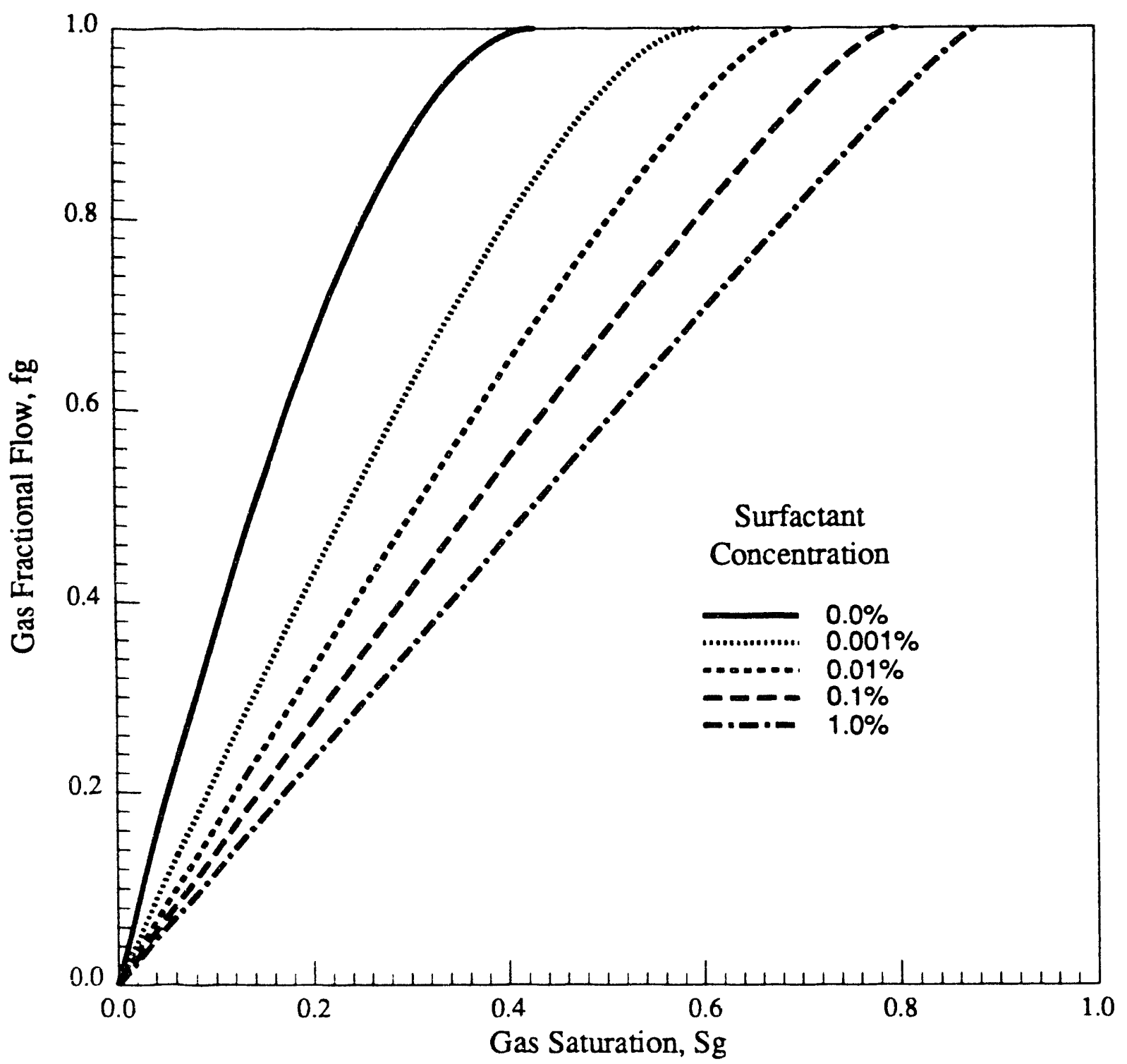

Figure 5.35: Summary of the Fractional Flow Curves from Experiments 
To obtain the velocity of each saturation, the derivative of the fractional flow curve is required. From Equation 5.11, it is:

$$
\frac{d f_{g}}{d S_{g}}=\frac{\bar{S}_{g}-S_{g}}{W_{i}^{2}} \frac{d W_{i}}{d S_{g}}-\frac{\frac{d S_{g}}{d W_{1}} \frac{d W_{i}}{d S_{g}}-1}{W_{i}}
$$

where $\left(d \bar{S}_{g}\right) /\left(d W_{i}\right)$ can be obtained by differentiating Equation 5.10 :

$$
\frac{d \bar{S}_{g}}{d W_{i}}=\left(\frac{d b}{f^{2}}-\frac{a}{f}\right)\left[\ln \left(b-\frac{f}{W_{i}}\right)-\ln (b)+\frac{b W_{i}}{b W_{i}-f}-1\right]
$$

and $\left(d W_{i}\right) /\left(d S_{g}\right)$ comes from the differentiation of Equation 5.8:

$$
\frac{d W_{i}}{d S_{g}}=\frac{a}{\left(a-S_{g} b\right)^{2}}
$$

\subsection{Matching of Pressure Data}

Considerable effort was expended in attempts to match the pressure data obtained for these Eas displacement runs. The main problem with the pressure calculations was the lack of knowledge about the water relative permeability. Although it was demonstrated before that the commonly used relative permeability equations (Equations 4.1 and 4.2 ) do not work for these displacements, the water relative permeability was calculated from them anyway since there are no other better ways. Since this is a standard text-book pressure calculation, the procedures will nou be shown here for the purpose of brevity. Different values of the water exponents $n_{w}$ were tried to see which ones would give decent matches of the pressure drops.

For Run 21 (gas displacing pure water), the pressure drop first goes up and comes down and the data are erratic (Figure 5.36). No matter what $n_{w}$ value was used, it was impossible to match the shape of the pressure data. The calculated pressure drops increased after breakthrough while the data shows the contrary behavior.

In Run 31 (gas displacing $0.001 \%$ surfactant-laden solution), an $n_{w}$ value of 4.0 matched fairly well at the breakthrough time, although the match was not good at any other times (Figure 5.37).

Towards a higher surfactant concentration, in Run 32 (gas displacing $0.01 \%$ surfactantladen solution), a good match was fonnd at $n_{w}=4.2$ for early times, but the calculated breakthrough time was not matched well at all (Figure 5.38). Also the predicted behavior after breakthrough did not match the experiment. 


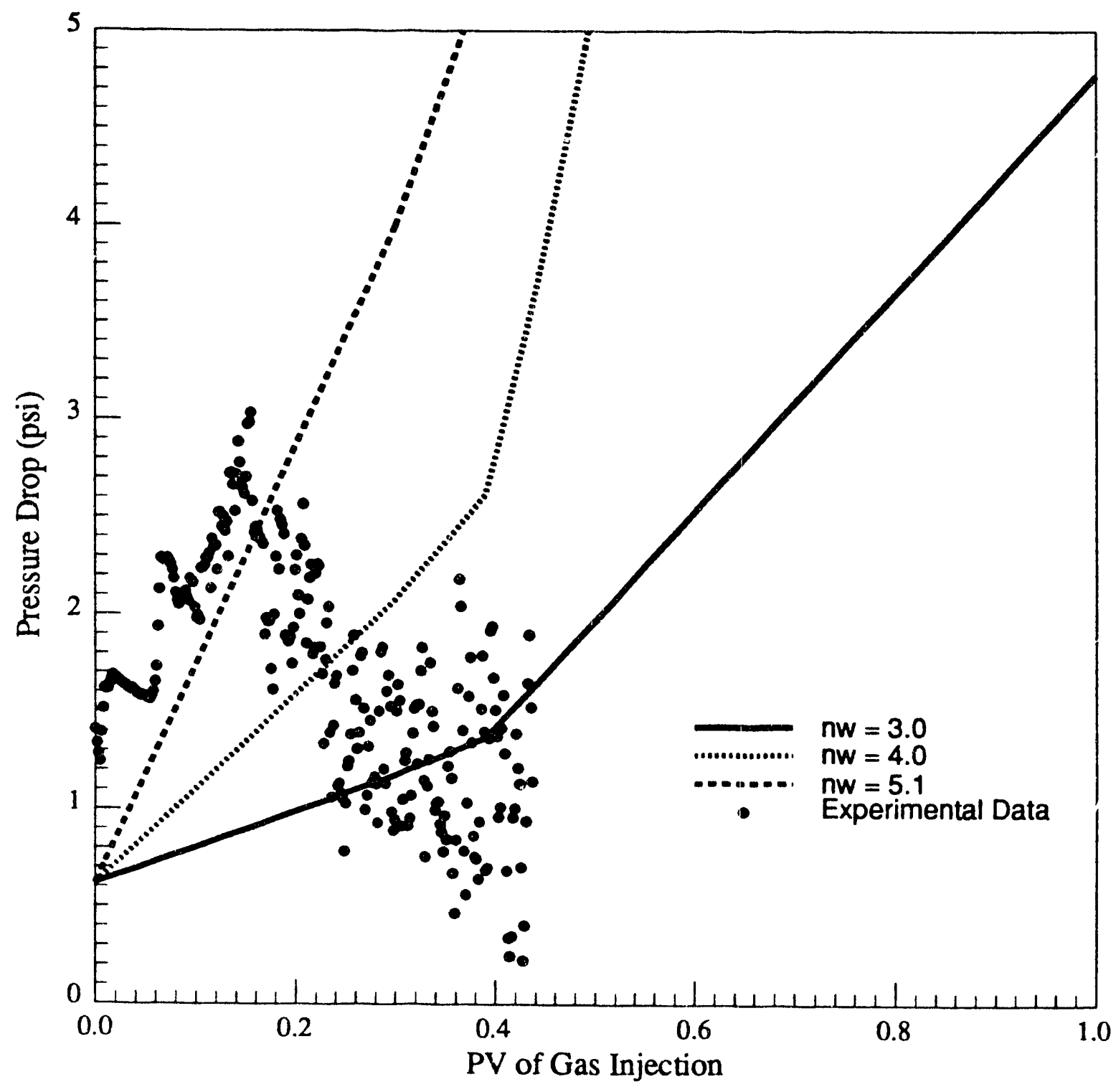

Figure 5.36: Pressure Calculations for Run 21 (0.0\% Surfactanı Concentration) 


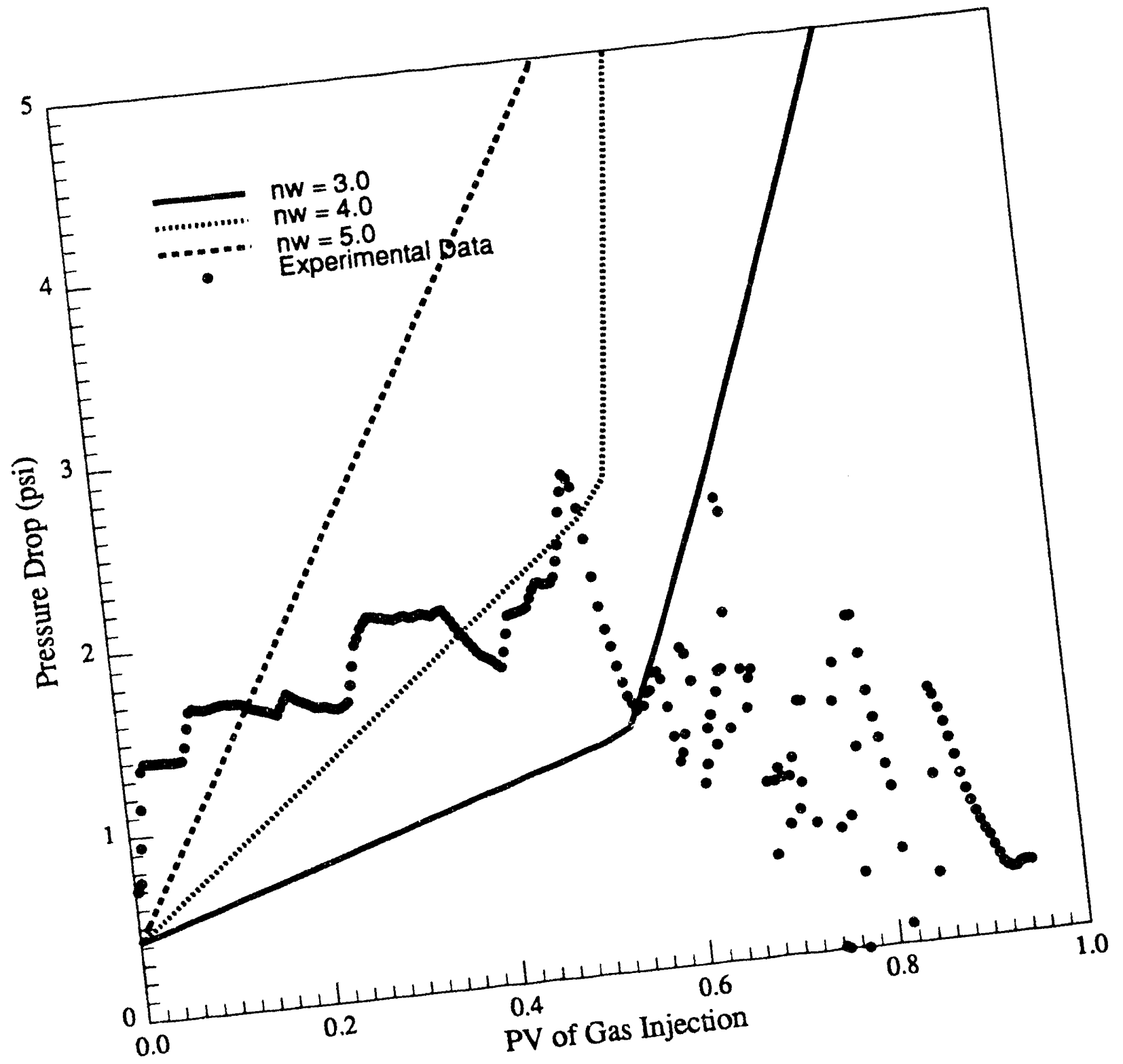

$(0.001 \%$ Surfactant Concentration) Figure 5.37: Pressure Calculations for Run 


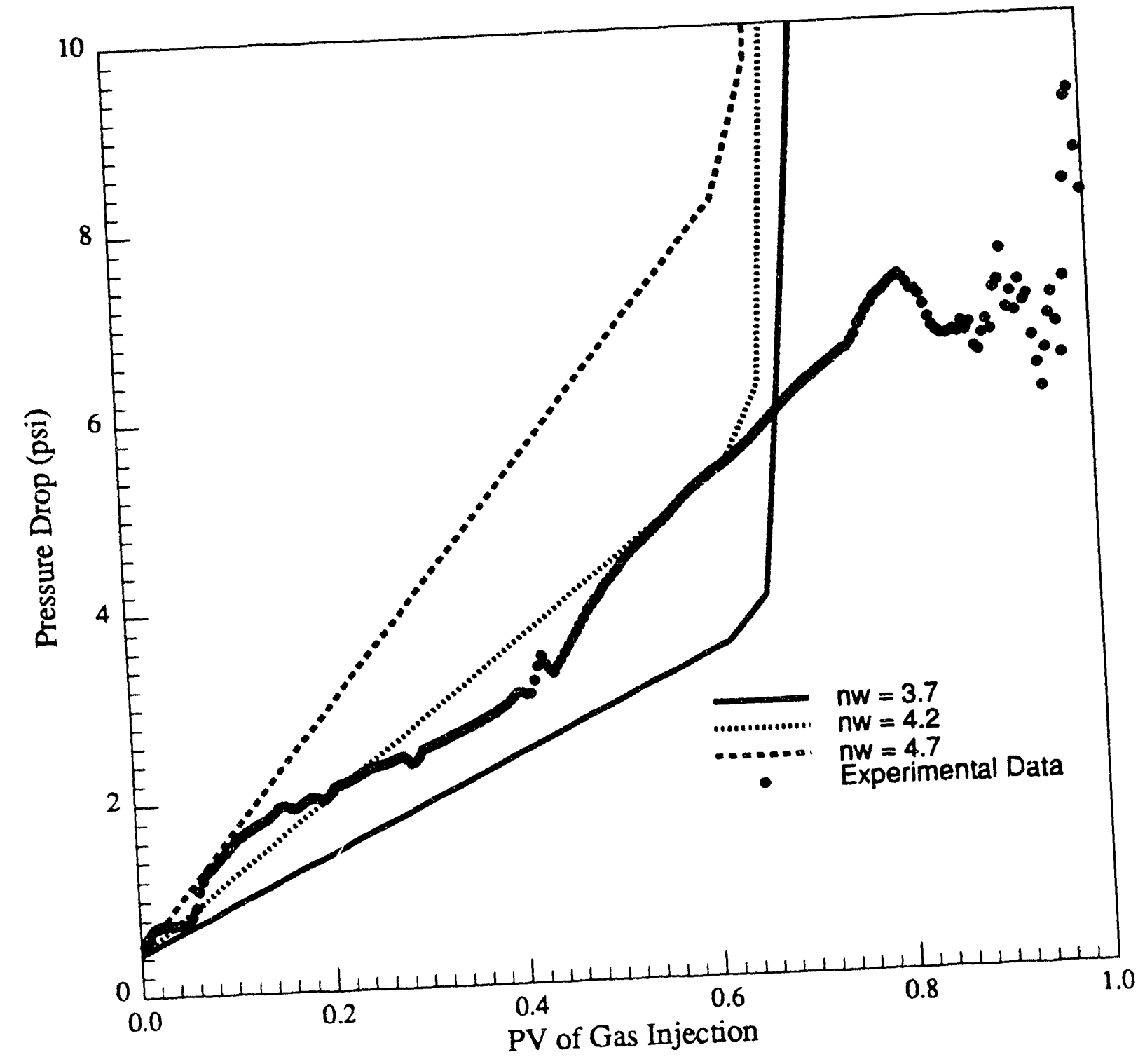

Figure 5.38: Pressure Calculations for Run 32 (0.01\% Surfactant Concentration) 
In Run 35 (gas dispiacing $0.1 \%$ surfactant-laden solution), a still higher $n_{w}$ value of 4.7 matched both the breakthrough time and the pressure data (Figure 5.39) up to the breakthrough time, but not afterwards.

Also in Run 34 (gas displacing $1.0 \%$ surfactant-laden solution), an $n_{w}$ value of 5.1 matched the breakthrough time and the pressure data very closely (Figure 5.40). Note that this $n_{w}$ value is much greater than what is normally found in the literature.

From all the pressure matches above, it can be seen that while it is easy to find an $n_{w}$ value to match the pressure data at high concentrations, it is difficult to do so at low concentrations. The trouble here is that, although it is possible to match some of the runs at high concentrations, the $n_{w}$ value tends to be higher as the concentration goes higher. So, it is impossible to find one value for $n_{w}$ that would match all the runs. This again shows that the relative permeability equations usually used in the literature do not apply in this work. Clearly further work needs to be done on this subject, with more careful pressure instrumentation to learn what is going on in these displacements. 


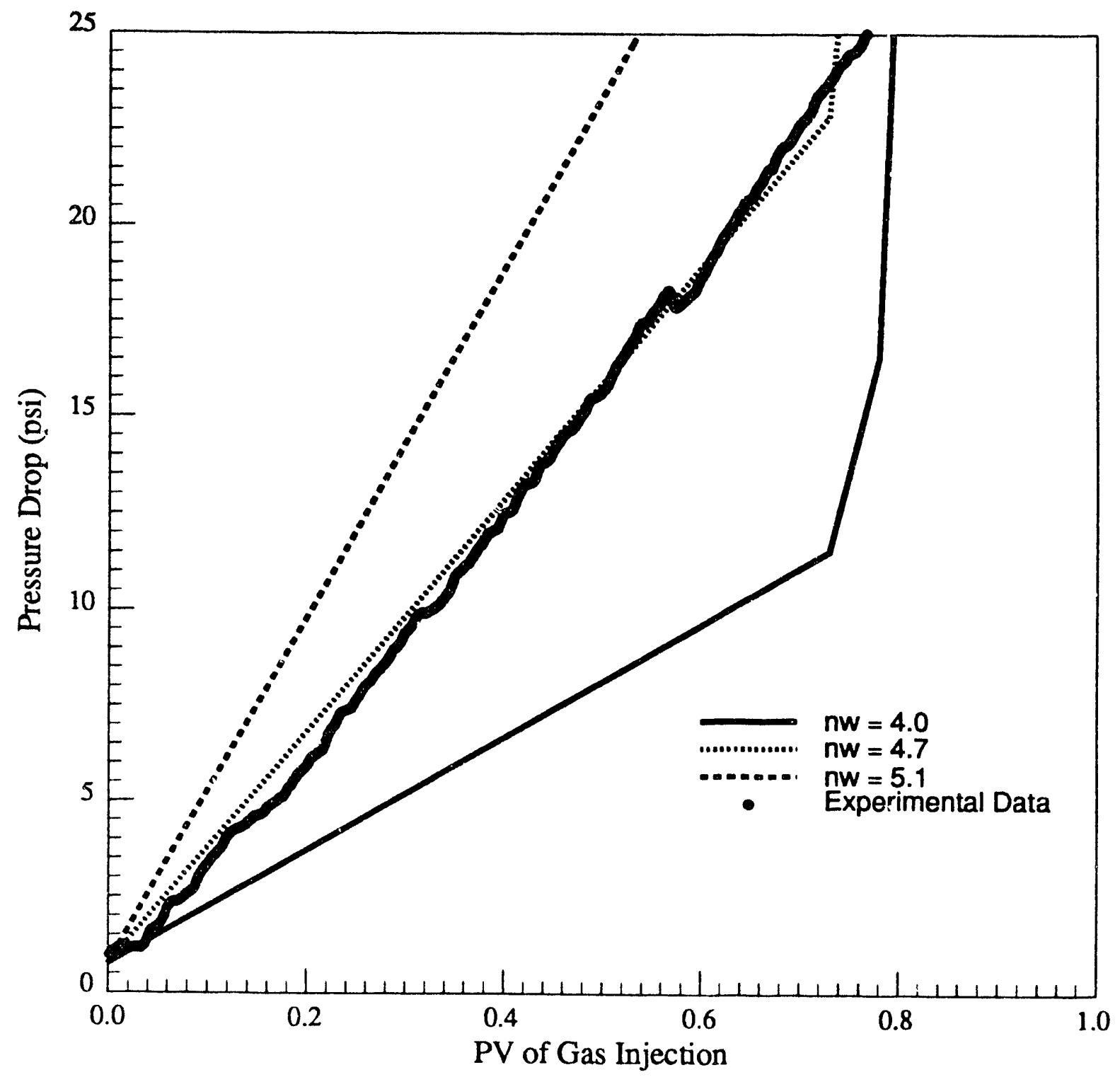

Figure 5.39: Fressure Calculations for Run 35 (0.1\% Surfactant Concentration) 


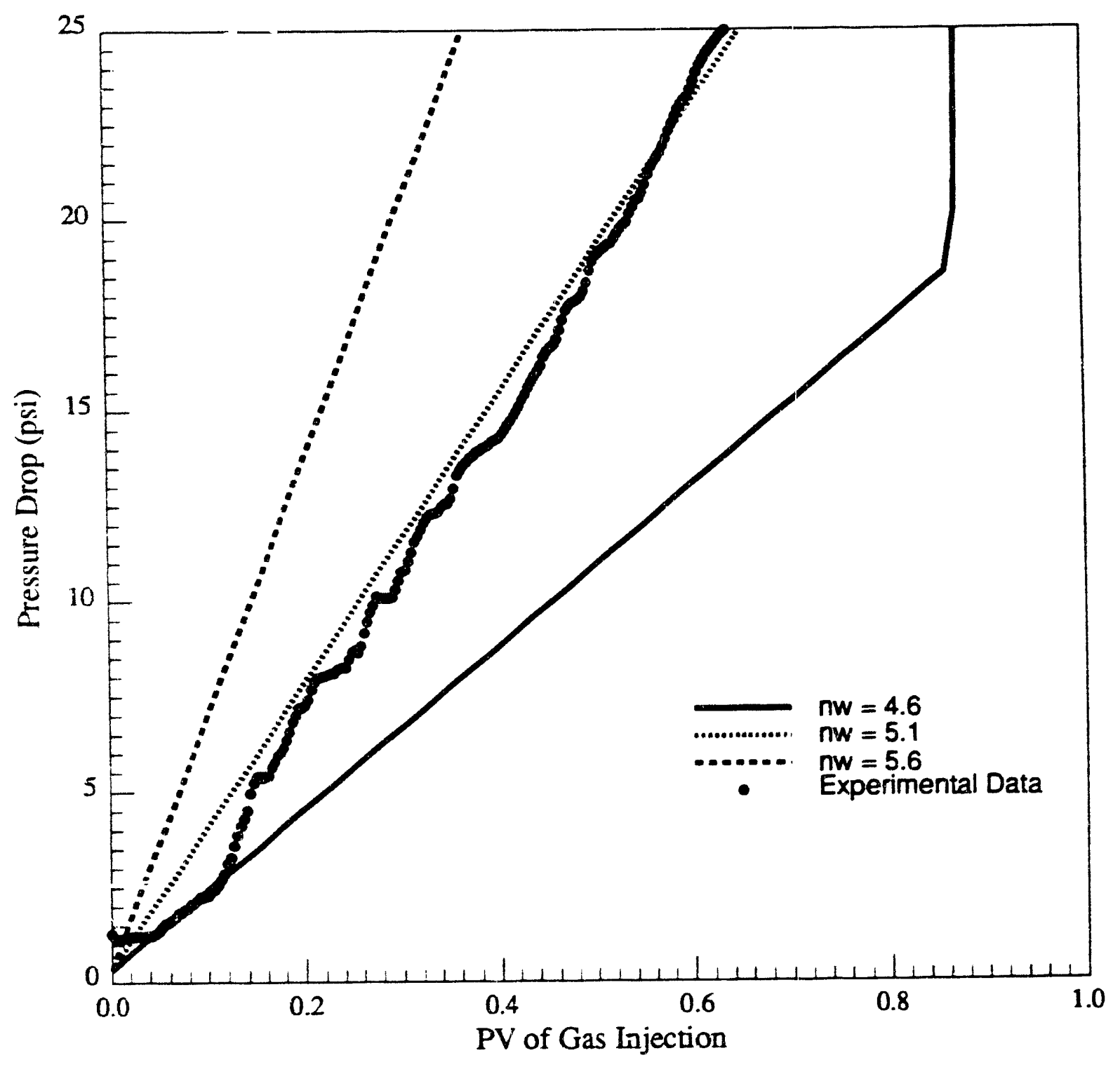

Figure 5.40: Pressure Calculations for Run 34 (1.0\% Surfactant Concentration) 


\section{Chapter 6}

\section{Results of Foam Displacements}

Having obtained the necessary basic displacement parameters, the next step is to match the theoretical model with the data from foam displacements. Experimental runs were made to obtain data for foam flow in the porous medium. Then the displacement foam model developed in Chapter 4 was used to match the data. This last series of experiments were the core of this work aimed at finding the foam flow mechanism in porous media, where foam is injected into sand packs initially saturated with distilled water.

\subsection{Summary of Foam Flow Experiments and Observations}

A summary of the experimental runs of foam displacement is presented in Table 6.1. The foam quality nas set at $90 \%$ for all these runs. The foam injection rates were 0.01 and $1.0 \mathrm{cc} / \mathrm{min}$. It should be noted here that the foam flow rates shown in the table were calculated rates from the anticipated pressure conditions. The real flow rates, calculated from the experiments, were slightly different and will be presented later. The surfactant concentration was either at 0.1 or 1.0 weight percent. The absolute permeability of the sandpack varied from 6.5 to 7.0 Darcies. The porosity fell in the range of 32 to $35 \%$. Only the last two runs, Run 41 and Run 42, rendered sufficient data for a complete analysis. The rest of the runs served to accumulate experience observing foam flow in a porous medium. They also helped develof a systematic experimental procedure for the last two runs in terms of CT Scanning techniques and other data gathering. It is realized that the useful runs were made only with two different surfactant concentrations as the variable. There are many other factors affecting the foam flow process, such as the foam quality, injection rates. type of surfactant, presence of oil, nature of the porous medium, and permeability. So more russ should be made in the future to take these variables into account. 


\begin{tabular}{|c|c|c|c|c|c|}
\hline $\begin{array}{c}\text { Run } \\
\text { No. }\end{array}$ & $\begin{array}{c}\text { Injection } \\
\text { Velocity(cc/min) }\end{array}$ & $\begin{array}{c}\text { Surfactant } \\
\text { Concentration (wt\%) }\end{array}$ & $\begin{array}{c}\mathrm{K} \\
(\text { Darcy) }\end{array}$ & $\begin{array}{c}\phi \\
(\%)\end{array}$ & $\begin{array}{c}\text { Length of } \\
\text { Sandpack }\end{array}$ \\
\hline 1 & 0.1 & 0.1 & 6.5 & 35 & 24 inches \\
\hline 3 & 1.0 & 0.1 & 7.0 & 33 & 24 inches \\
\hline 4 & 1.0 & 1.0 & 6.7 & 32 & $2 c_{c}$ inches \\
\hline 7 & 1.0 & 0.1 & 6.5 & 32 & 24 inches \\
\hline 9 & 1.0 & 0.1 & 6.5 & 32 & 24 inches \\
\hline 10 & 1.0 & 1.0 & 6.9 & 32 & 24 inches \\
\hline 41 & 1.0 & 1.0 & 6.7 & 33 & 46 inches \\
\hline 42 & 1.0 & 0.1 & 6.7 & 32 & 46 inches \\
\hline
\end{tabular}

Table 6.1: Experimental Runs of Foam Displacement

The pressures measured from the pressure taps were plotted versus the distance from the inlet of the sandpack. As an example, the pressure distribution for Run 4 is presented in Figure 6.1. The surfactant useci for this run was AOS 1618 with a concentration of $1.0 \%$. The foam injection rate was $1.0 \mathrm{cc} / \mathrm{min}$. The circles on the pressure curves indicate the foarn front visually observed from the outside of the sandpack.

The pressure profile for Run 9 is presented in Figure 6.2. The surfactant concentration was $0.1 \%$. Comparing the two figures, one can see that a higher surfactant concentration produced a greater pressure drop for the same amount of foam injected. In both cases, it is obvious that the pressure gradient behind the foam front is much higher than that ahead of the front. This shows the high apparent viscosity of foam. The pressure gradient increases with the displacement of original fluid with foam. However the relationship between pressure gradient and volume injected is very complex and is dependent on many factors.

As mentioned before, the liquid saturation is linearly proportional to the CT number measured at a cross section. Again it should be noted that the CT numbers given were the mean values. There was a standard deviation attached to each CT number. A greater standard deviation means a more heterogeneous saturation distribution in the cross section. To illustrate this concept, Figure 6.3 gives a graph of CT number (liquid saturation) versus pore volumes injected for Run 4 at location 1 ( 1 inch from the inlet), with the standard deviation shown as vertical bars for each data point. As is seen, the standard deviations for water-and gas-saturated sandpack cross sections were small, indicating that the cross section was fairly homogereous. When the foam displaced part of the in-situ water, the standard deviation became considerably larger, which suggests that the foam flow was not a piston-like displacement. Visual observations of C.T pictures also confirmed channeling of the gas. 


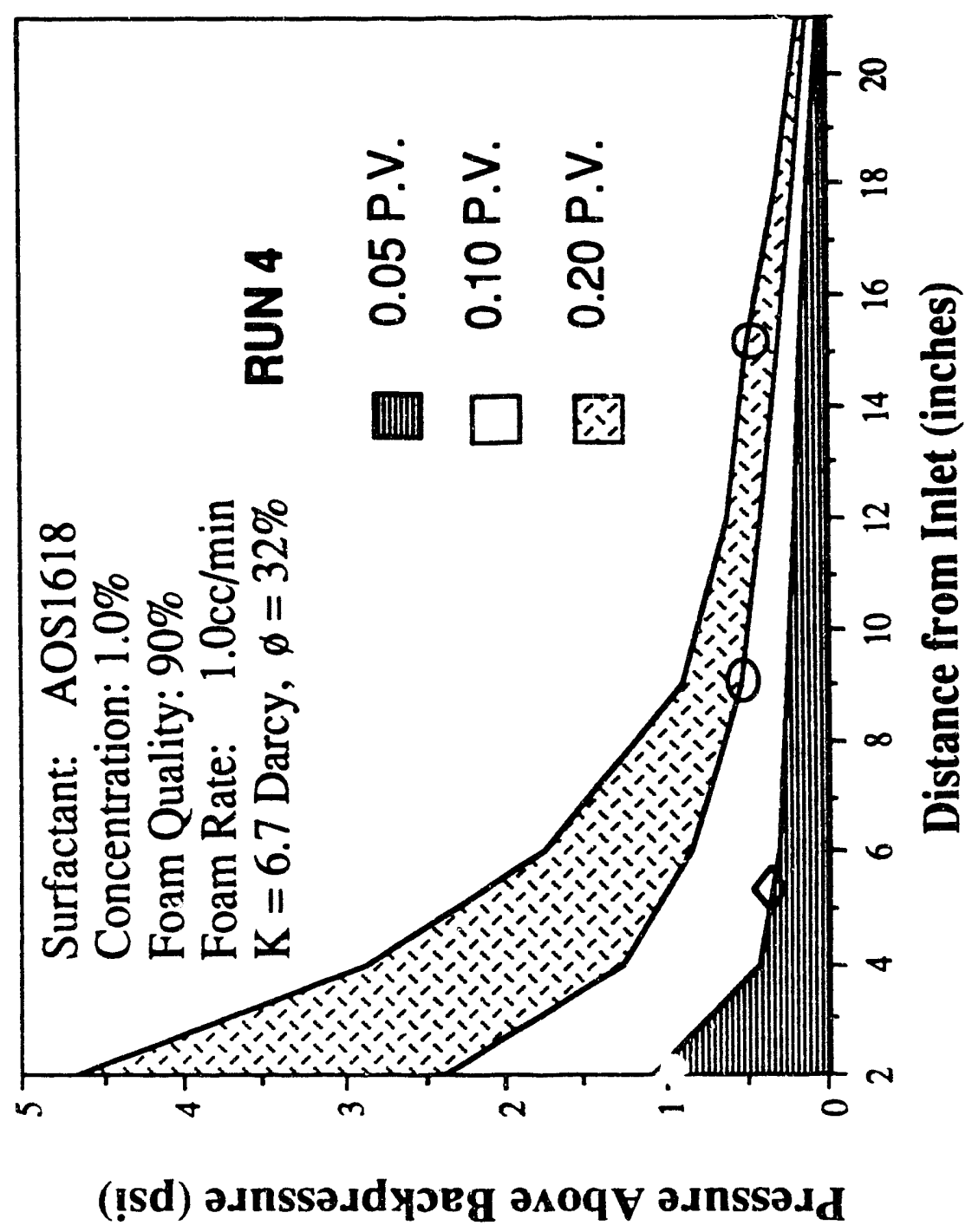

Figure 6.1: Pressure Distribution versus PV Injected (Run 4) 


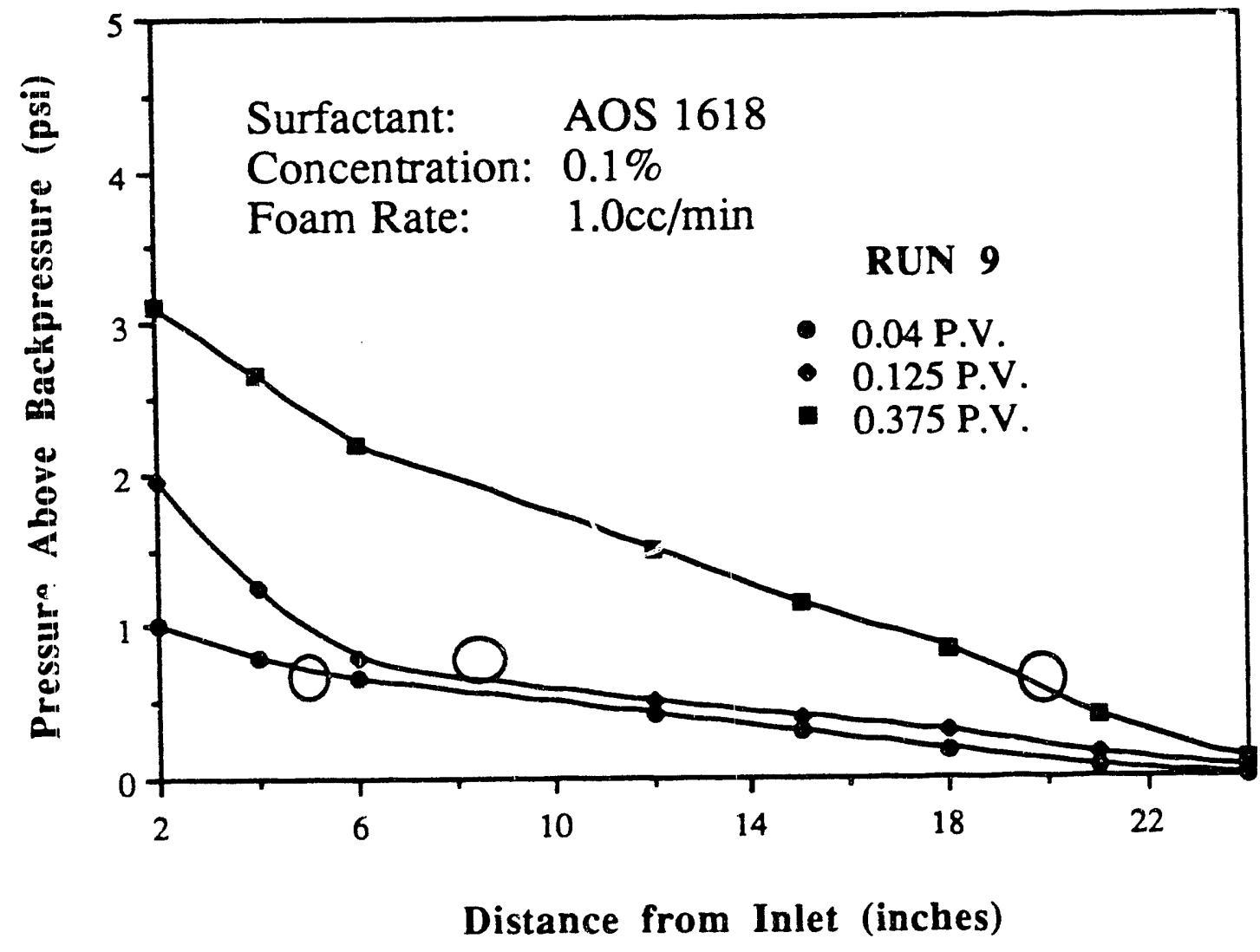

Figure 6.2: Pressure Distribution versus PV Injected (Run 9) 


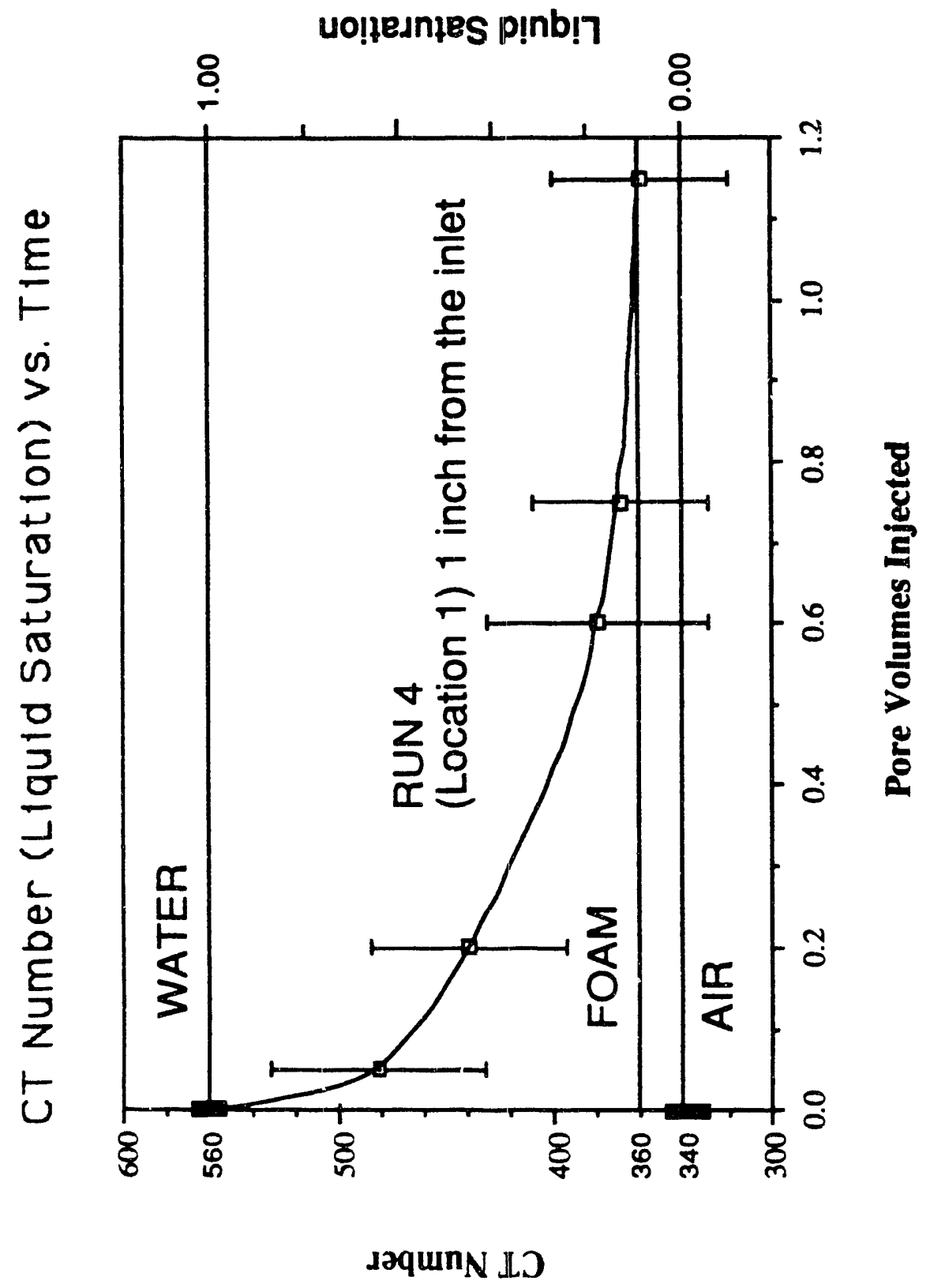

Figure 6.3: CT Detection of Heterogeneities (Run 4) 
To compare the data from each cross section, a common factor must be used. To accomplish this, the volurne of foam injected was converted into an equivalent pore volume (EPV) which was based on the pore volume from the inlet to the cross section concerned. Naturally, the EPV to the middle of the sandpack is half of the total pore volume, and the EPV at the end is the total pore volume. As an example, Figure 6.4 shows the CT numberEPV relationship for Run 9 at the cross section location 1 ( 1 inch from the inlet). To get a smooth curve out of the experimental data, various fitting methods were tried. It seems a good method was a power function for the relationship between liquid saturation and EPV. Different locations and experimental conditions resulted to different relationships. Just to give an example, as can be seen in Figure 6.4, the CT number-EPV relationship at Location 1 was found to be:

$$
N_{C T}=473 \times E P V^{-0.059}
$$

The match in this case was quite good, but this type of power formula resulted in poor matches in many cases. It was found that a still better match could be obtained with a Langmuir-type formula if the liquid saturation was converted to gas saturation, as discussed earlier in Chapter 5 and also later in this chapter.

Figure 6.5 shows a sequence of CT pictures while foam was displacing in-situ water in Run 10 at cross section Location 1 ( 1 inch from the inlet). In the beginning, the cross section was homogeneous(Figure 6.5(a)) when saturated with water. Then foam of $90 \%$ quality ( $1 \%$ surfactant concentration) was injected into the sandpack at the rate of $1 \mathrm{cc} / \mathrm{min}$. At a later time (6 minutes after foam injection, Figure 6.5(b)) gas was starting to invade the cross section. The darker part of the picture is the gas in the foam. As can be seen clearly, the foam flow was not a piston-like displacement. There was much fingering and channeling, particularly near the center portion of the sandpack.

As time passed, the cross section was filled with more foam. The foam blocks the gas channels and then displaces more of the liquid from the cross section(Figure 6.5(c) and (d)). Note that Figures $6.5(\mathrm{a})$ and $6.5(\mathrm{~b})$ have a window level of 500 while Figures $6.5(\mathrm{c})$ and $6.5(\mathrm{~d})$ have a window level of 350 . Basically, a higher window level gives greater contrast. At 36 minutes (Figure 6.5(c)), foam had displaced almost all the water from the cross section which would have been too dark to be viewed at the window level of 500 . Through experience, 350 was chosen as the new window level to visualize the data. At the later stage of displacement, the saturation change slowed down. So the last two CT pictures are hard to distinguish from each other with the naked eye.

It was found that for the surfactant concentrations studied ( 0.1 and 1.0 weight $\%)$, the higher surfactant concentration produced a better and faster displacement. For a lower concentration, the foam in the front broke but could not easily coalesce again, probably due 


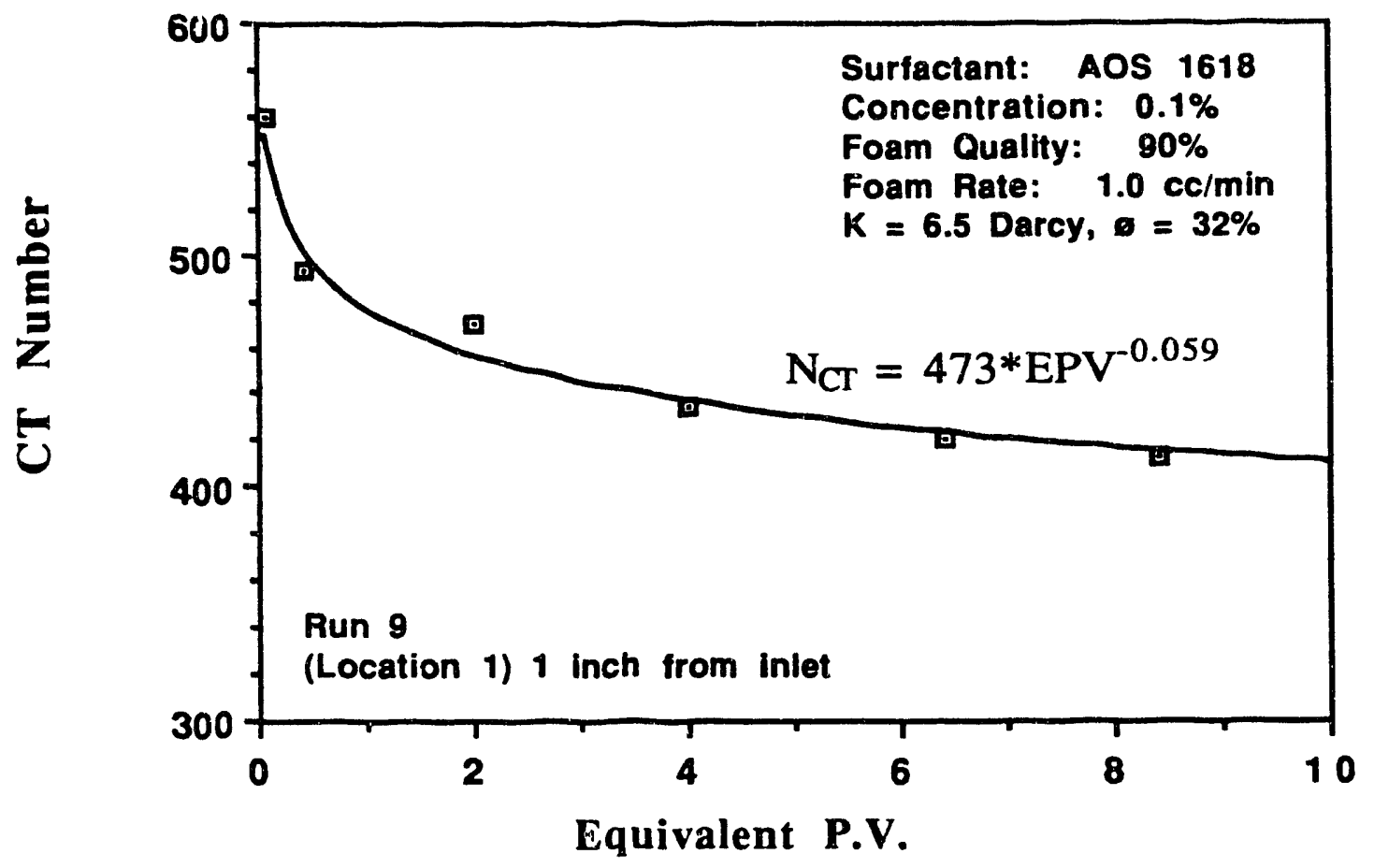

Figure 6.4: Relationship between CT Number and EPV Injected (Run 9, Location 1) 


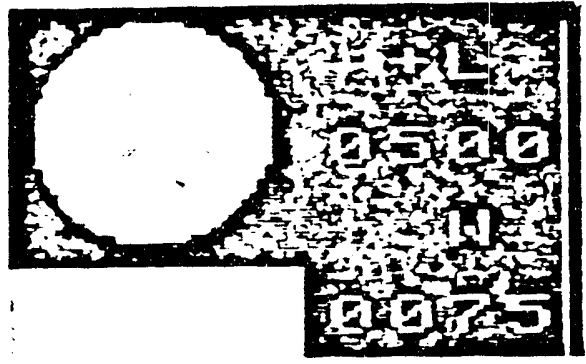

(a) Saturated with water

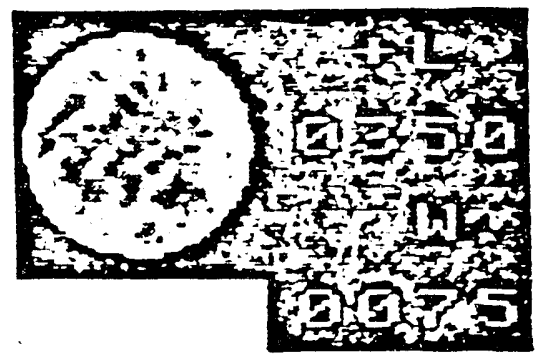

(c) 36 minutes after roam injection

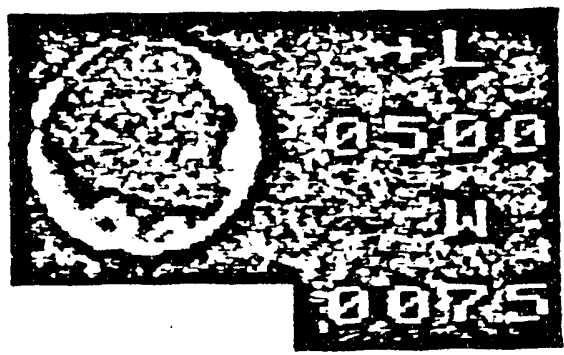

(b) 6 minutes añer foam injection

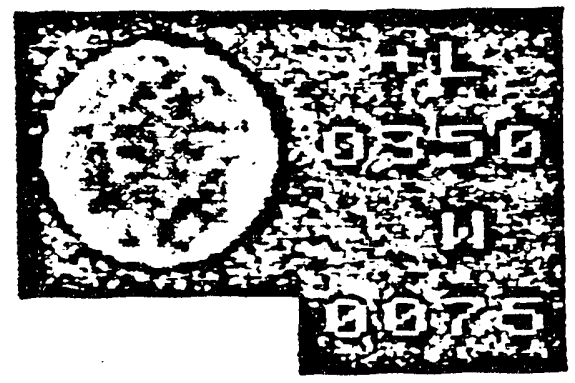

(d) 60 minutes aner foam injection

Figure 6.5: CT Pictures from Run 10 (Location 1) 
to adsorption of the surfactant on to the porous medium. In this case, the gas fingering was more severe and the foam (gas) breakthrough was faster. Thus, as might be expected, complete displacement required more pore volume throughput of foam.

\subsection{Experimental Results of Final Runs}

The final runs were made with foam displacing distilled water in long sandpacks. The saturation histories for Run 41 are shown in Figure 6.6. In this run, foam of $90 \%$ quality at $1 \%$ surfactant concentration was injected at a rate of $0.87 \mathrm{cc} / \mathrm{min}$ foam. The saturation histories at four different locations $(4.7,14.7,30.5$ and 41.2 inches from the inlet) were graphed versus EPV of foam injected. Unlike the Buckley-Leverett displacements shown for the gas displacement runs (Figures 5.1, 5.3, 5.5, 5.9, and 5.11), the saturation histories did not fall on top of each other. Also the pressure behavior as seen earlier in Figures 6.1 and 6.2 and next, in the figures that will be discussed, contradicts the simple Buckley-Leverett displacement theory. If it had conformed to t'se Buckley-Leverett theory, the pressure gradients behind the fronts would have been interrelatable throughout the displacement process.

The pressure responses at the pressure taps are graphed in Figure 6.7. From the pressure behavior, it can be seen that initially the foam did not cause a large pressure drop, apparently because it separated into gas. Only after many pore volumes of foam injection did the pressure drop increase significantly.

Run 42 was made at $0.1 \%$ surfactant concentration and a foam flow rate of $0.87 \mathrm{cc} / \mathrm{min}$, with all the other conditions the same as in Run 41 . The saturation histories and pressure responses are shown in Figures 6.8 and 6.9. The high pressure responses for the first few minutes were due to the experimental procedures of maintaining the system at a higher pressure before the foam was introduced. The pressure drops should have started at small values and gradually risen, in a way similar to Run 41 . The same trend of movement was seen as in Run 41 except that the displacement was a little less efficient and the pressure drop a little smaller. However, there was not a big difference between these two runs in the early stages. The reason behind this behavior will be discussed later. 


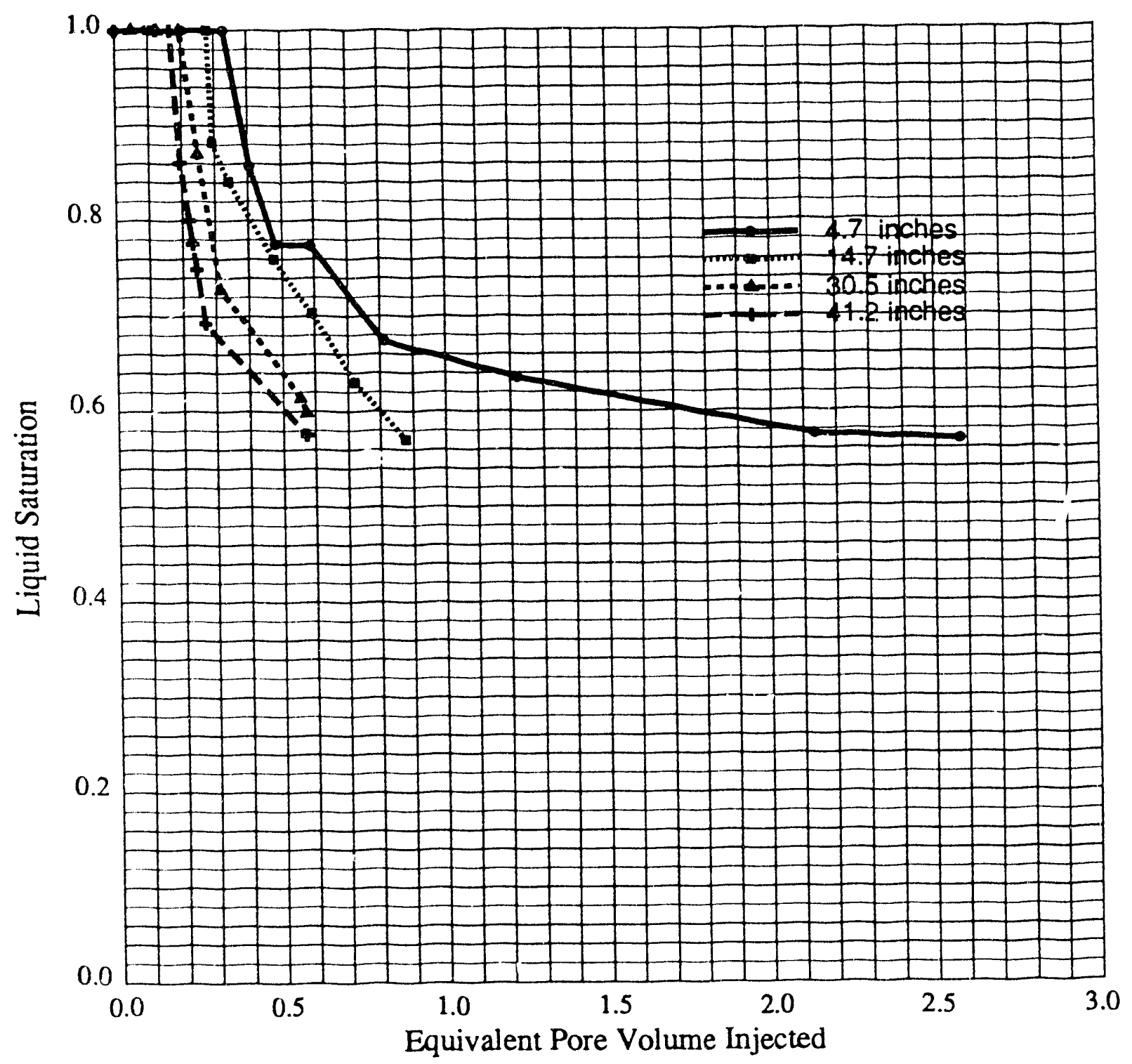

Figure 6.6: Saturation Histories for Run 41 ( $1 \%$ Surfactant Foam Displacing Water) 


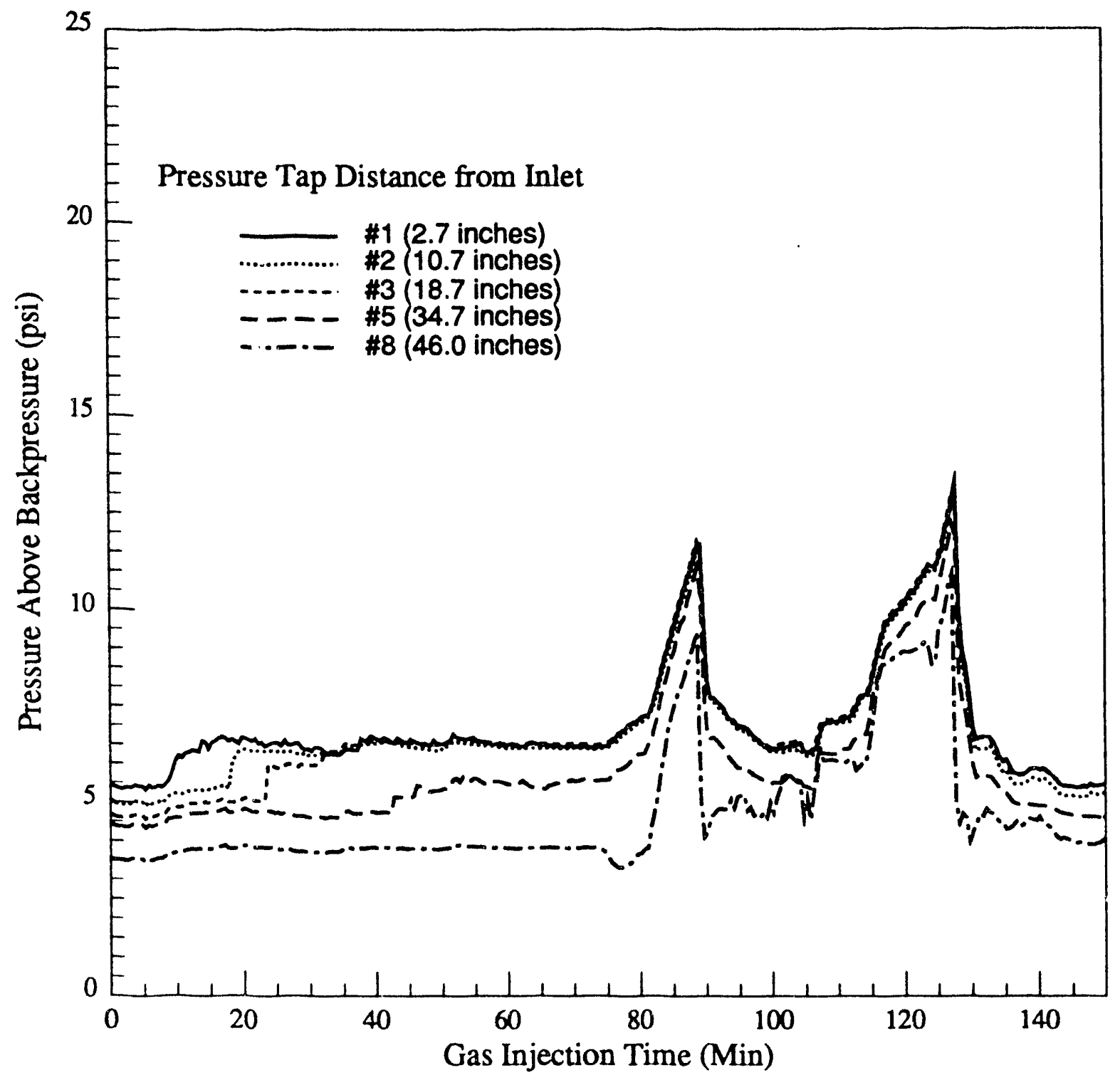

Figure 6.7: Pressure Responses for Run 41 ( $1 \%$ Surfactant Foam Displacing Water) 


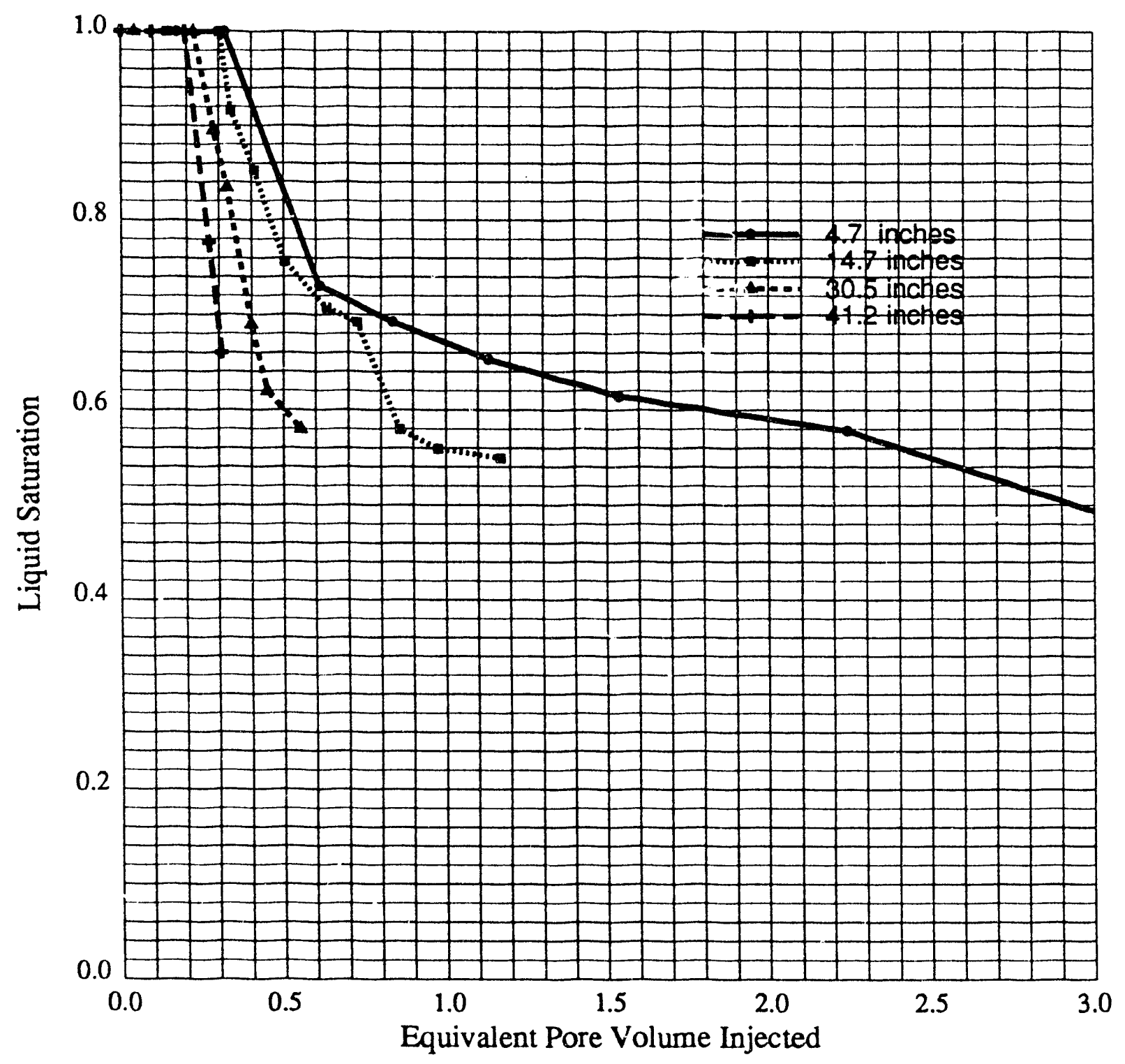

Figure 6.8: Saturation Histories for Run 42 ( 0.1\% Surfactant Foam Displacing Water) 


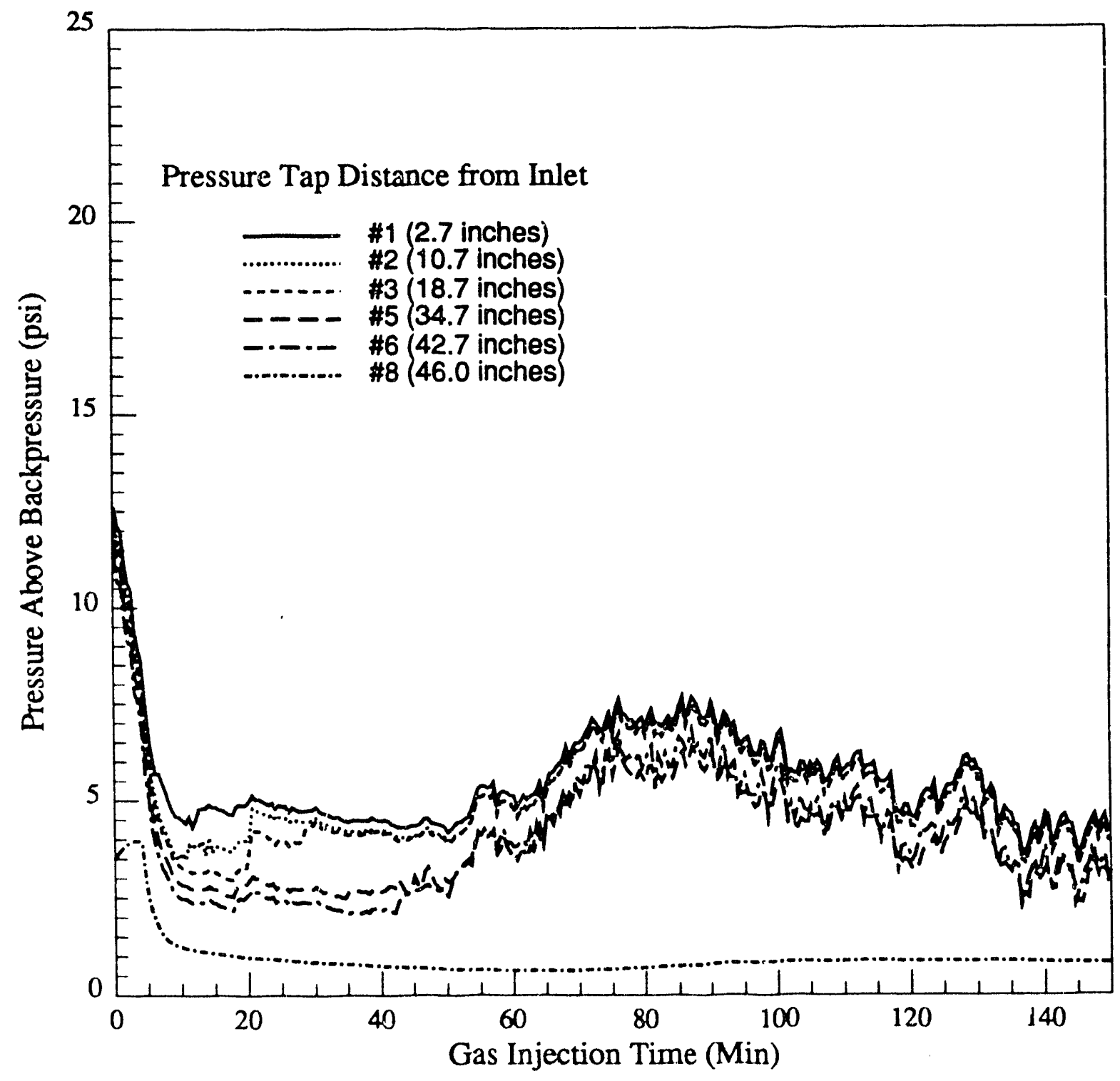

Figure 6.9: Pressure Responses for Run 42 (0.1\% Surfactant Foam Displacing Water) 


\subsection{Theoretical Matching of Experimental Data}

To match the experimental data on foam flow, the theoretical procedures described in Chapter 4 , Equations 4.4 through 4.17 were used. A computer program written to follow those procedures. listed in Appendix C, first interpolated t? e parameters obtained from the gas displacement runs. described in Chapter 5 , and then calculated the fractional flow curves and the saturation histories.

Corresponding to Run 41 , the calculated fractional flow curves for surfactant concentrations ranging from 0 to $1.0 \%$ are shown in Figure 6.10 . The first curve on the left side in the figure corresponds tc pure gas with no surfactant. The last one on the right shows the fractional flow curve for the highest surfactant concentration (1.0\%) and hence the highest apparent viscosity. Not all the fractional fium curves are shown in this figure for the sake of visualization. In the computer run to get the theoretical results de cribed later. twenty-nine shocks were used. That is. twenty-nine fractional sow curves were calculaced. The locations of these fractional flow curves were interpolated according the the parameters found in Chapter 5.

Then the saturation history at the outiet was calculated acrording to the displacement theory including the effect of adsorption (Equations 4.4 through 4.17). This history is graphed in Figuie 6.11. The calculated saturation history was from the displacement theory based on shocks. That is why the resulting curve is not smooth in Figure 6.11. Realizing that this saturation shape was due to using a finite num of shocks, while there are really an infinite number. it is known that the saturation hi= ory should be smooth. So the saturation history was smoothed by taking the mid-point average of all neighboring points, and the resulting saturation history (smoothed) along with that from the displacement theor: are graphed in Figure 6.12. Comparing Figure 6.12 with the experimental data of Rin 41 in Figure 6.6. it can be seen that the calculated saturation history matches well with that near the inlet ( 4.7 inches). Other saturation histories wi. ${ }^{1}$ be matched using the mixing effect, Equation 4.17 later in this chapter.

\subsubsection{The Effect of Surfactant Adsorption on Foarn Flow}

As described in Chapter 4, the displacement theory also included the effect of surfactant adsorption on to the porous medium (Equations 4.4 through 4.17 ). If the frontal advance loss. $D$. was set to be zero in all these efuations. then the displacement theory would be one withont the effect of adsorption. The saturation histories were calculated accordingly. 


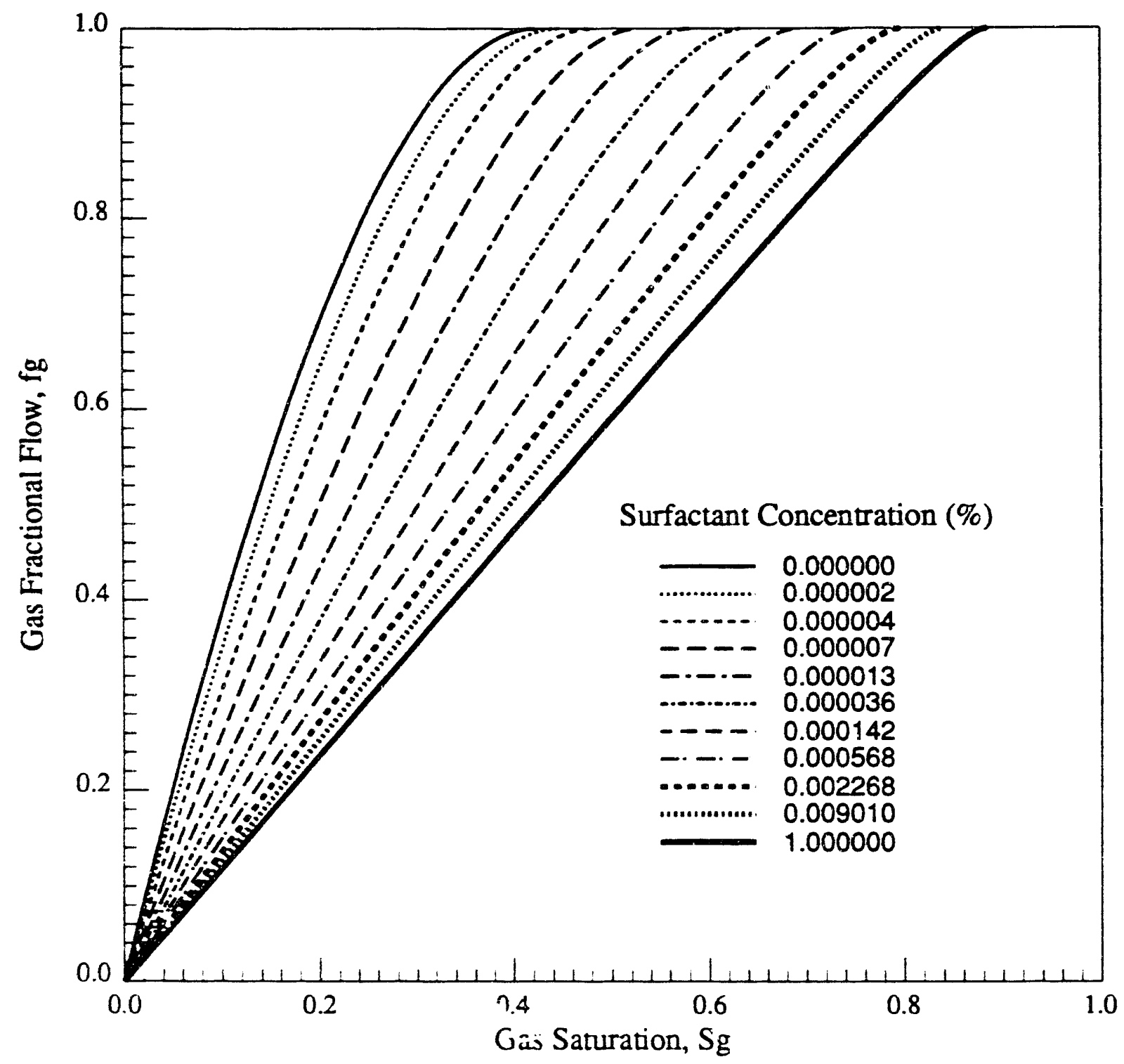

Figure 6.10: Calculated Fractional Flow Curves for Foam of 1.0\% Surfactant Concentration 


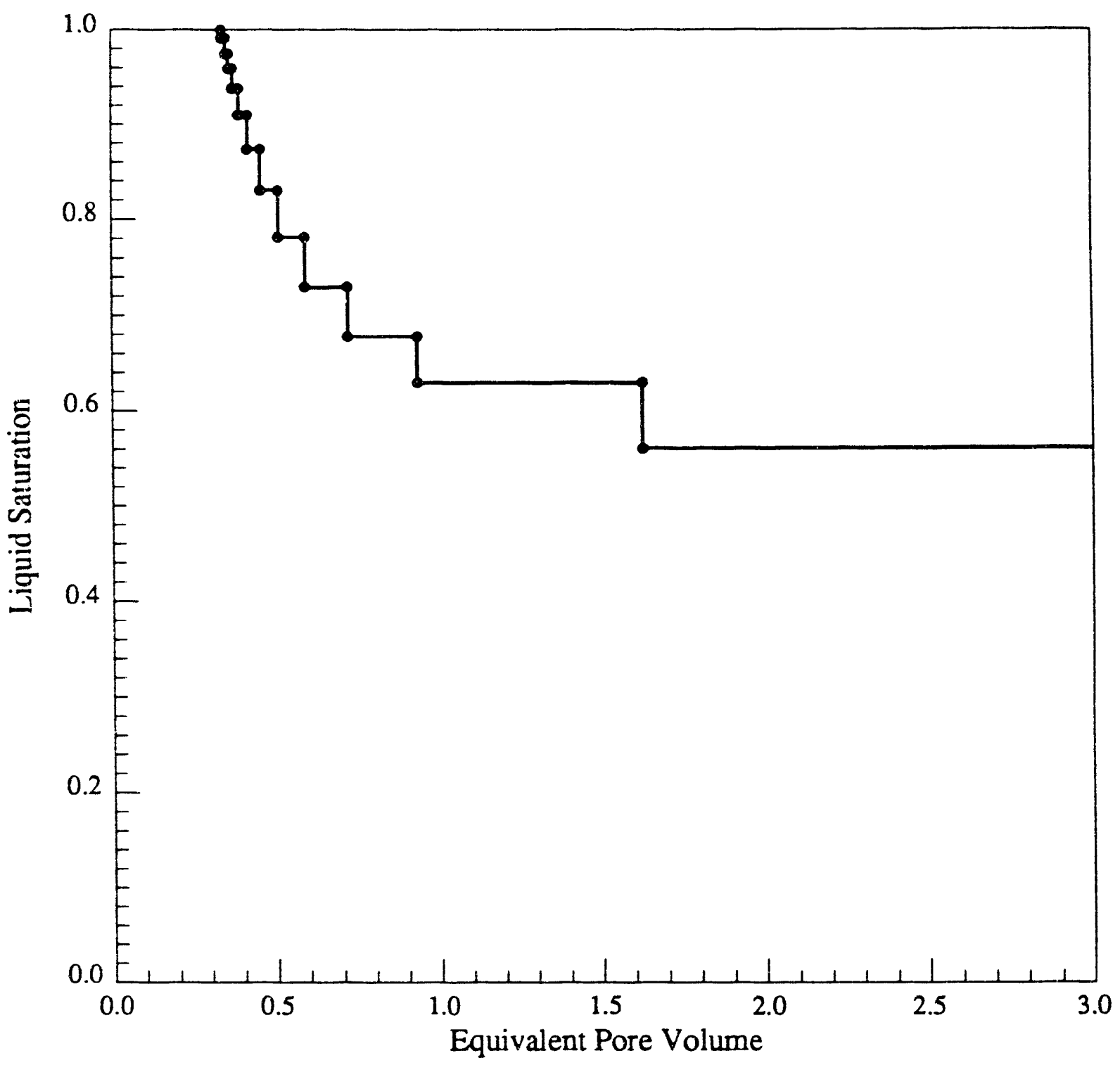

Figure 6.11: Calculated Saturation History for Foam of 1.0\% Surfactant Concentration 


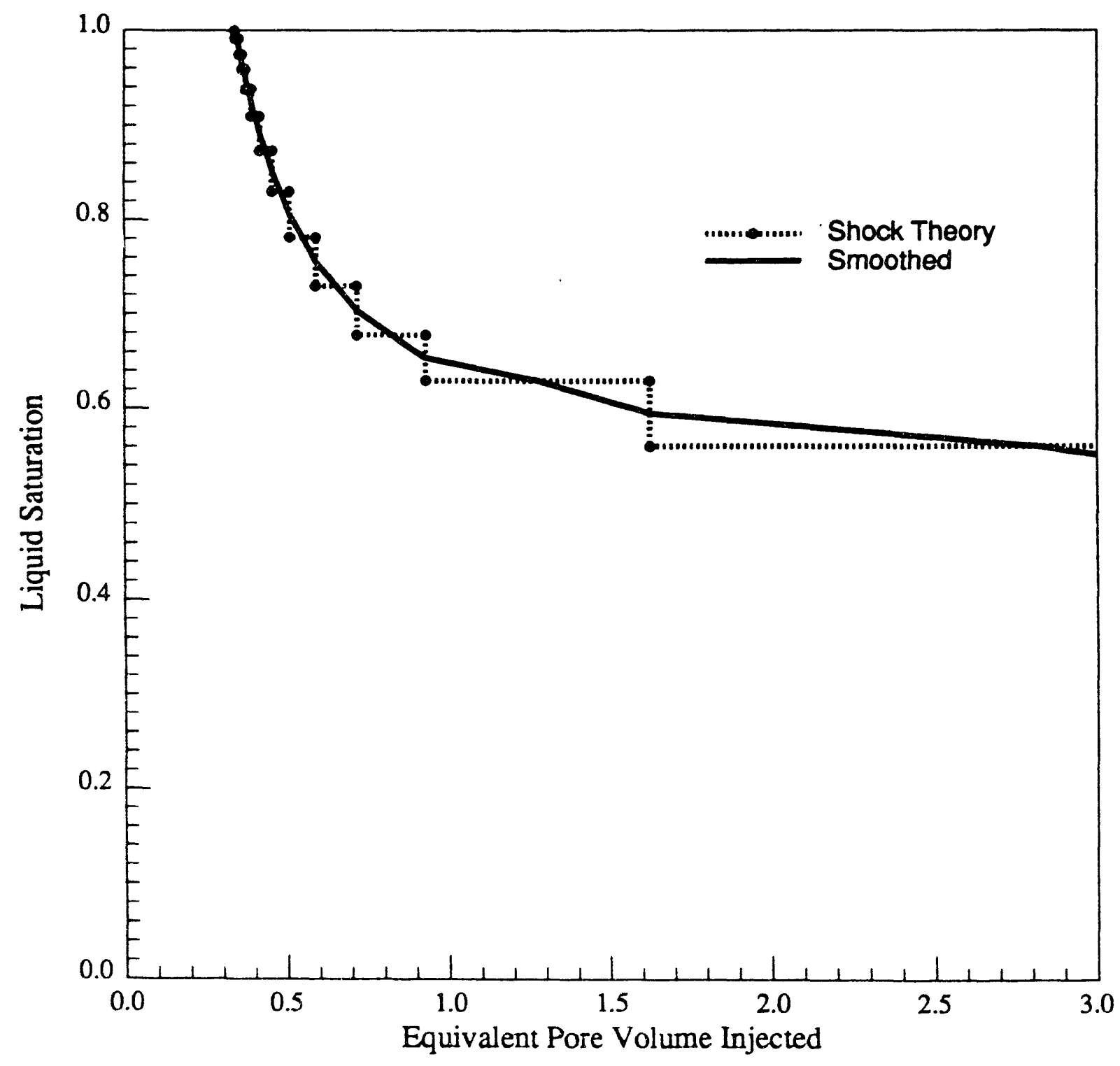

Figure 6.12: Calculated and Smoothed Saturation Histories for Foam of 1.0\% Surfactant Concentration 
The effect of surfactant adsorption was not significant, as shown in Figure 6.13 where the saturation histories were calculated with and without adsorption for the same location. A closer check on the saturation data shows that the surfactant adsorption retarded the first gas shock and gave later breakthrough, but had only a minor effect on the saturation history thereafter.

\subsubsection{The Effect of Mixing on Foam Flow}

To account for the mixing effect, the experimental saturation histories shown in Figure 6.6 were smoothed using Equation 5.3. The resultant gas saturation curves along with the experimental data are plotted in Figure 6.14. The matches were reasonably good.

After a little mathematical manipulation (similar to the derivation of Equation 5.7), the gas saturation can be expressed in terms of the distance traveled, $x$ :

$$
S_{g}=\frac{a\left(1-t_{B T} x\right)}{x+b\left(1-t_{B T} x\right)}
$$

Rearranging the above equation, the distance can also be expressed in terms of the saturation:

$$
x=\frac{a-S_{g} b}{t_{B T}\left(a-S_{g} b\right)+S_{g}}
$$

The saturation histories in Figure 6.14 are replotted in Figure 6.15. The method described in the theoretical part (Section 4.2) was followed to find the mixing coefficients as discussed next.

In Figure 6.15 , two saturations, 0.3 and 0.5 , were selected to find the mixing coefficients. According to the theory, the difference between the distances traveled by the two saturations is $\Delta X$. The distance traveled by the average saturation, 0.4 in this case, is $\bar{X}$. Since there were four locations for our experiments, we had four pairs of $\Delta X$ and $\bar{X}$ values that can be found from Figure 6.15. They are:

As was shown in Chapter 4 , plotting $\Delta X / X$ vs. $1 / \Delta X$ on a $\log -\log$ scale would give a rurwe which could be matched with the Brigham type curve to obtain the mixing coefficients. 


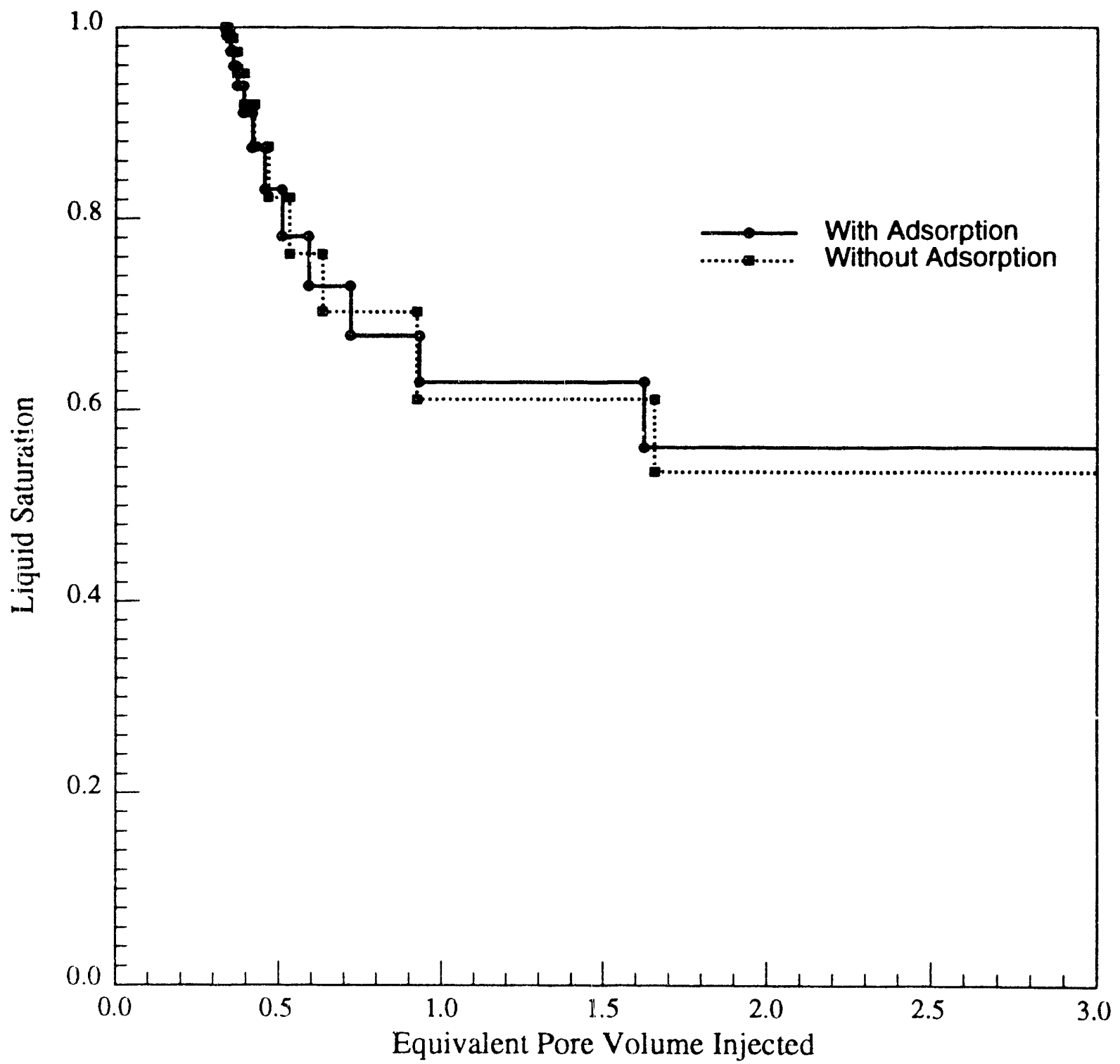

Figure 6.13: Effect of Surfactant Adsorption on Saturation Histories for Foam Flow of $1.0 \%$ Surfactant Concentration 


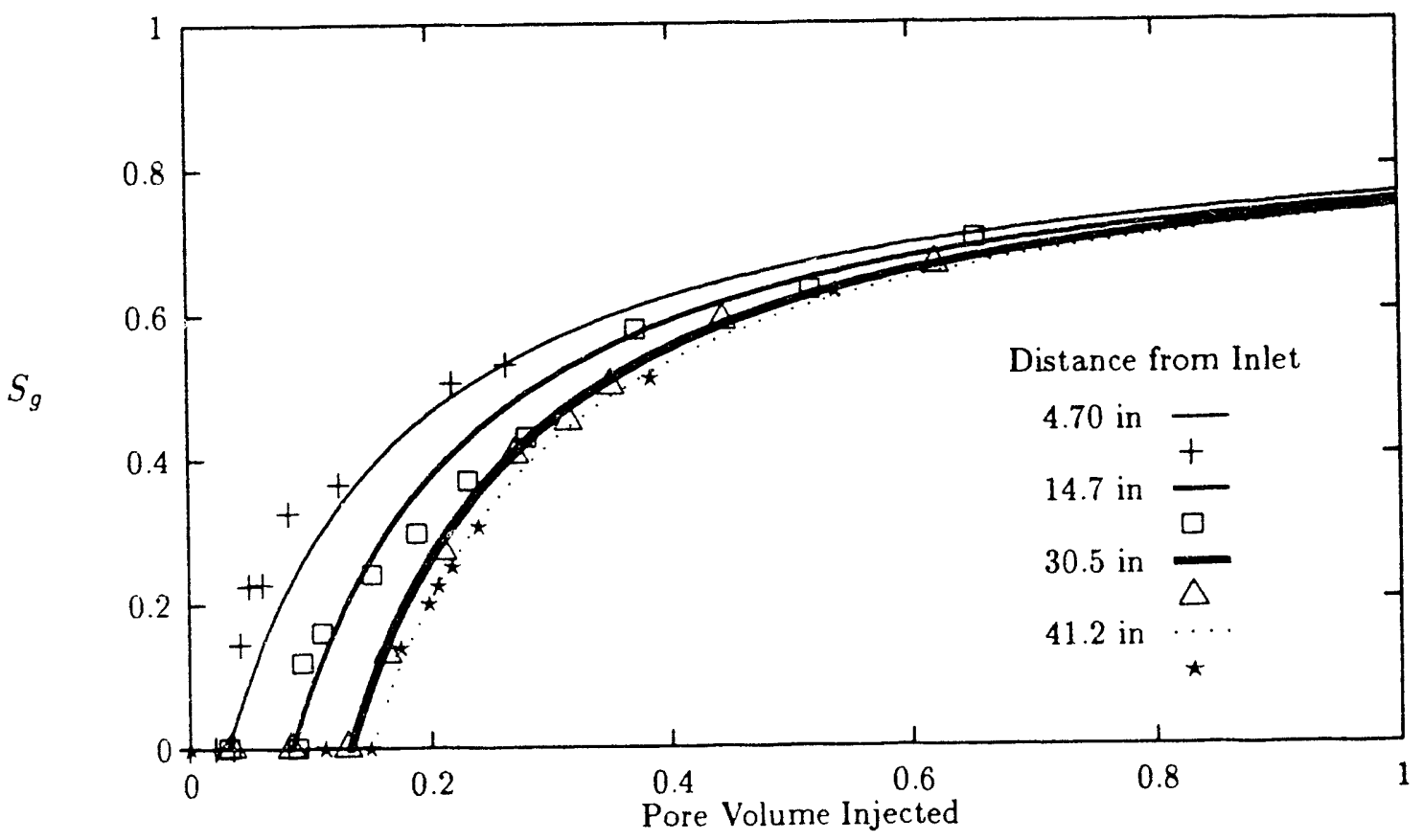

Figure 6.14: Saturation History Fits for Run 41 (1.0\% Surfactant Concentration) 


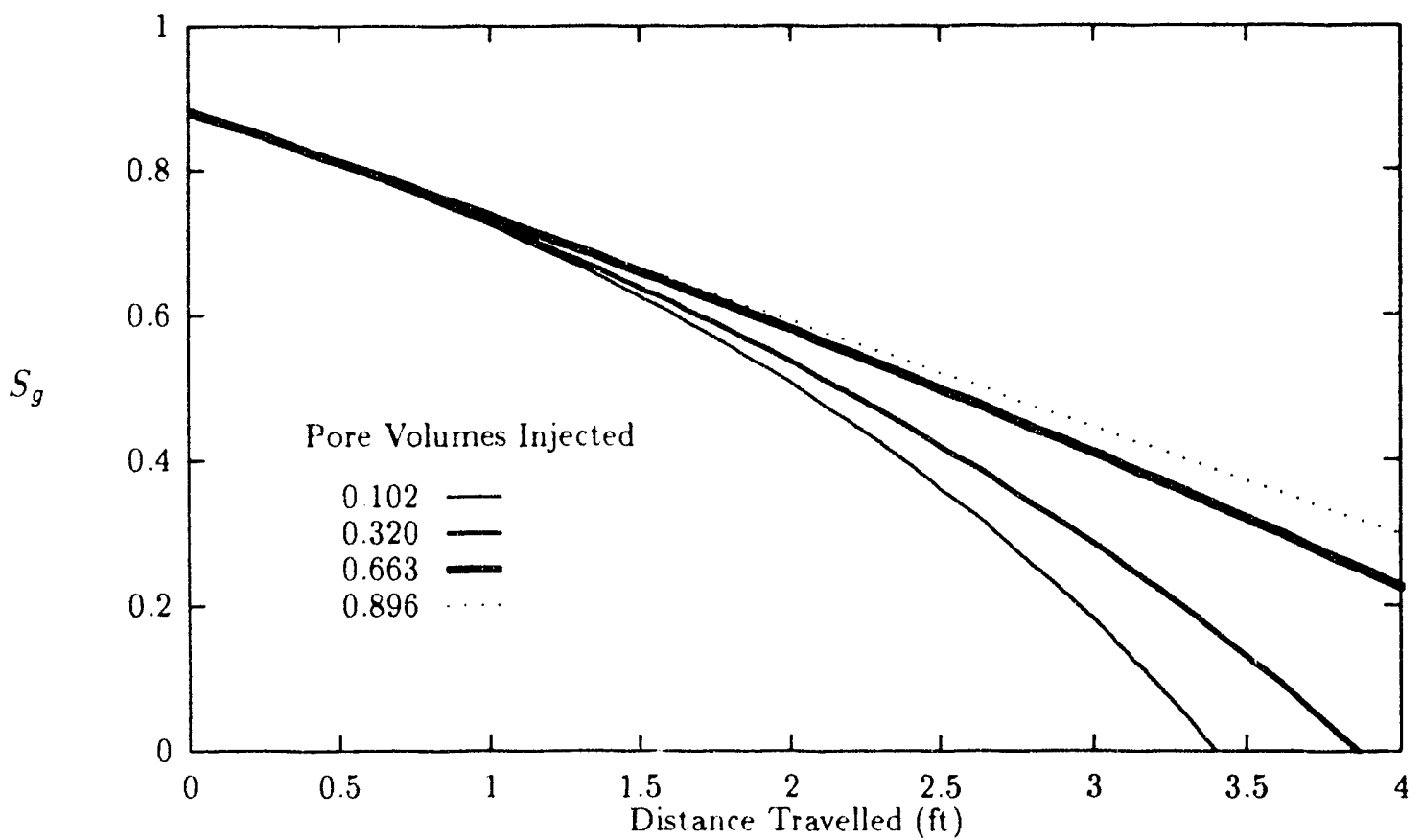

Figure 6.15: Saturation Profiles for Run 41 (1.0\% Suriactant Concentration) 


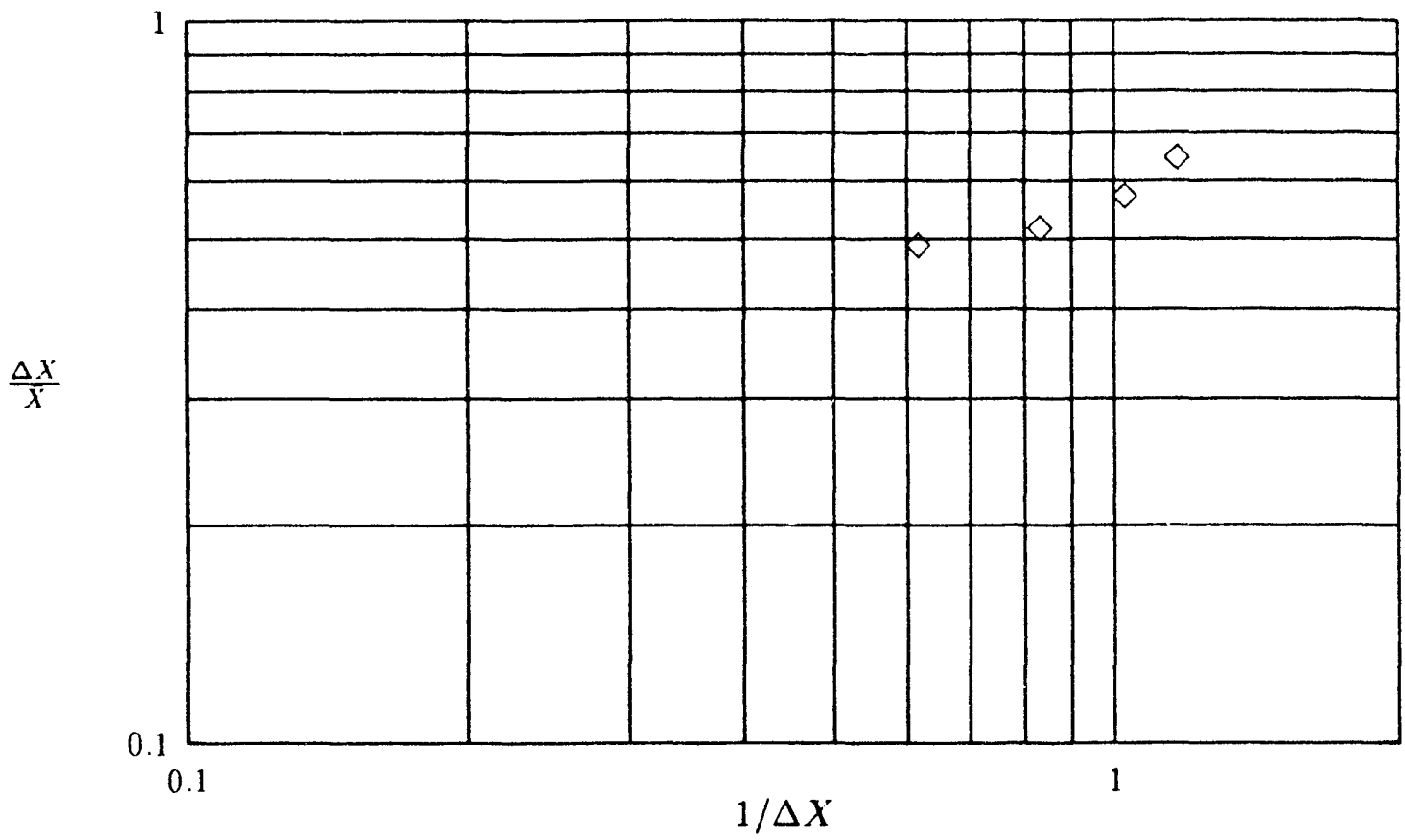

Figure 6.16: Type Curve Matching Data for Run 41

The data are shown in Figure 6.16. Since type curve matching is basically a graphical method of least squares fitting, the mixing coefficients $C$ and $\alpha$ can be found by a least squares fit to Equation 4.17 or Equation 4.16. This was the method used herein, and when Equation 4.17 was used, $C$ was found to be 0.290 and $\alpha, 0.138$. When Equation 4.16 was used, the value of $C$ was 0.298 and $\alpha$ was 0.135 , which are almost the same as those found from Equation 4.17. This means that one can choose either equation to fit the data and get about the same answers. By examining the range of data in Figure 6.16, it was realized that the mixing effect, although significant, did not dominate in this run. 


\subsubsection{Final Marching of Saturation Histories}

Equation 4.17 can be rearranged to get:

$$
\sigma^{2}-1.04675 C x \sigma-2.01029 \alpha x=0
$$

So $\sigma$ can be found as the root of the above quadratic equation:

$$
\sigma=\frac{1.04675 C x+\sqrt{(1.04675 C x)^{2}+4 \times 2.01029 \alpha x}}{2}
$$

For each location $x, \sigma$ can be calculated from the above equation. The ratio of the $C x$ value to $\sigma$ gives rise to the multiplier that should be used to account for the mixing effect for that location. If the first location ( 4.7 inches from the inlet) were to be the base for comparison, then the rest of the multipliers would be the ratio of $C x / \sigma$ for that location $(x)$ to that for the first (base) location. Details of these calculations are given in Appendix E. Applying these multipliers to the calculated saturation history for the first location (Figure $6.12)$ gives the final match of Run 41. The final smoothed match of the saturation history data is shown in Figure 6.17. As can be seen from the figure, the data for the location at 14.7 inches criss-cross the matched curve while the data at other locations follow their respective curves more closely. The matches, in conclusion, have two of the most important features: one was that they predirt well the breakthrough time, the other is that they cover the entire range of the data to take into account the mixing effect. Hence the final match is satisfactory.

The same procedures were used to match Run 42 in which $0.1 \%$ surfactant solution was used. The fractional flow curves are shown in Figure 6.18, where, again, only a few of the concentrations were graphed between 0 and $0.1 \%$. The calculated saturation history from the displacement theory is shown in Figure 6.19 and its smoothed version is shown in Figure 6.20 .

Much like what was done for Run 41 above, to get the mixing coefficients, saturation histories from the experimental run were smoothed using Equation 5.3 (Figure 6.21) and the saturation-distance relationship is shown in Figure 6.22. Then the $\Delta X$ and $X$ values were found from Figure 6.22. These values are shown in Table 6.3. 


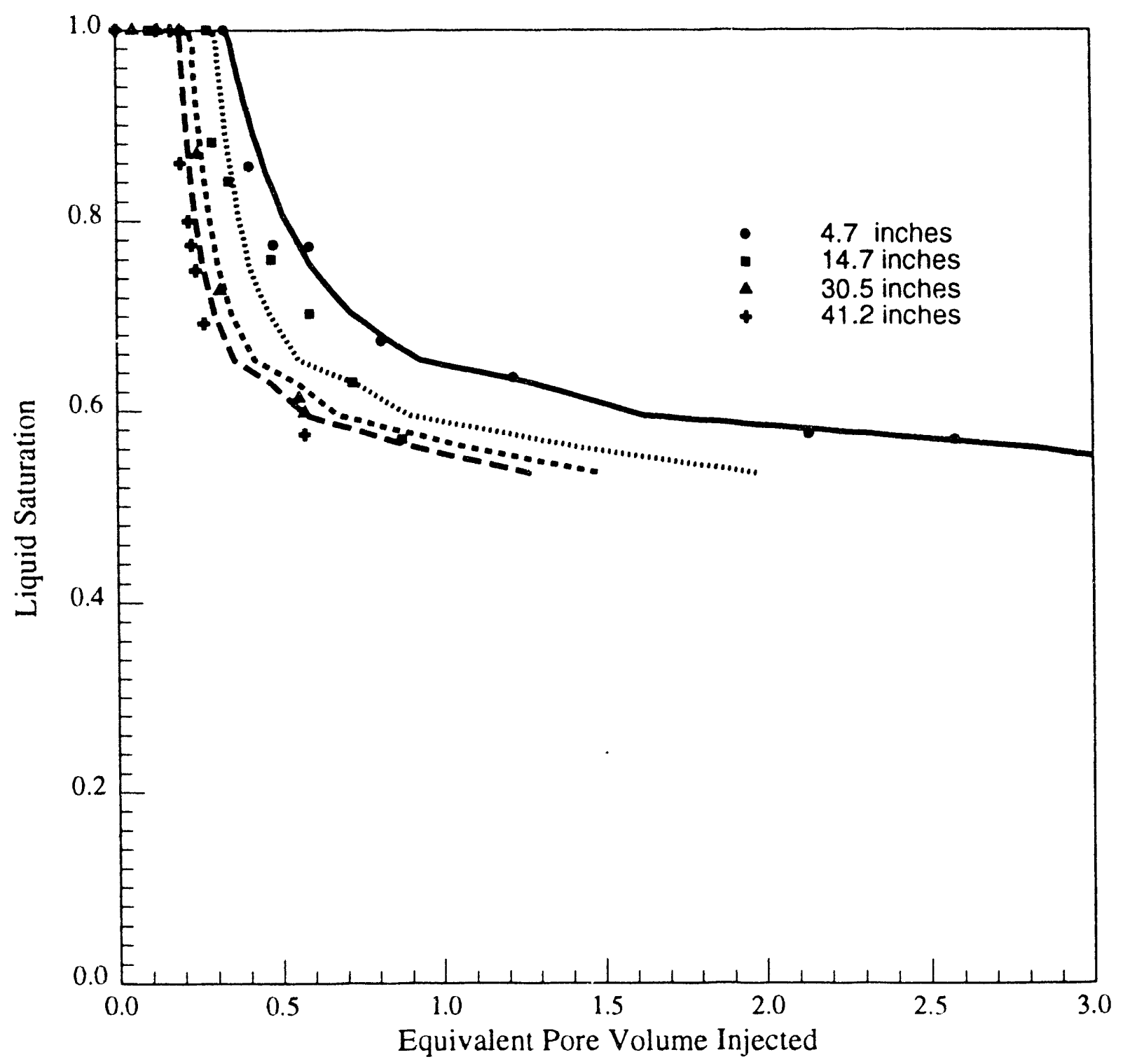

Figure 6.17: Final Saturation History Match for Run 41 (Flow of 1.0\% Surfactant Foam) 


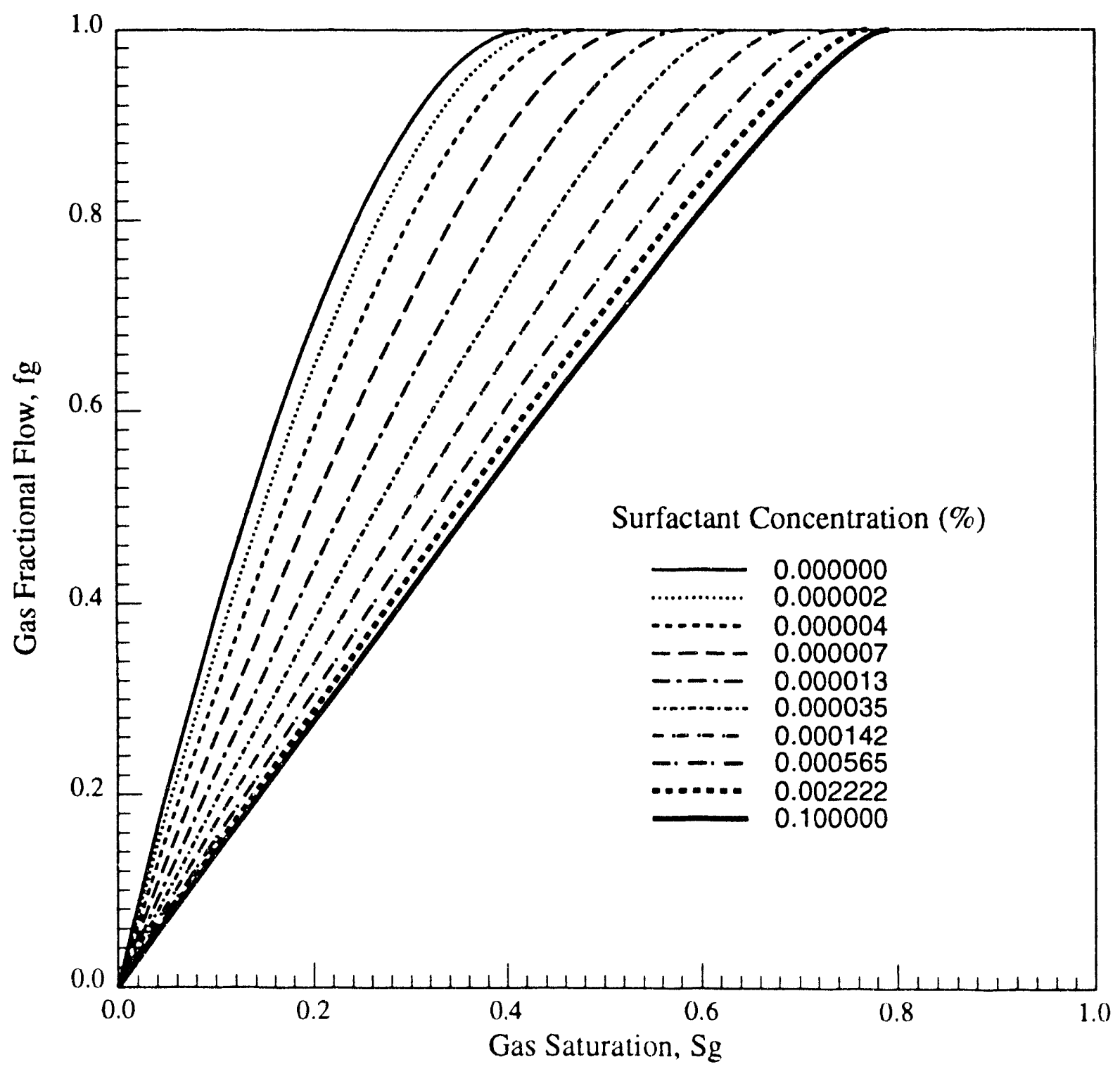

Figure 6.18: Calculated Fractional Flow Curves for Foam of 0.1\% Surfactant Concentration 


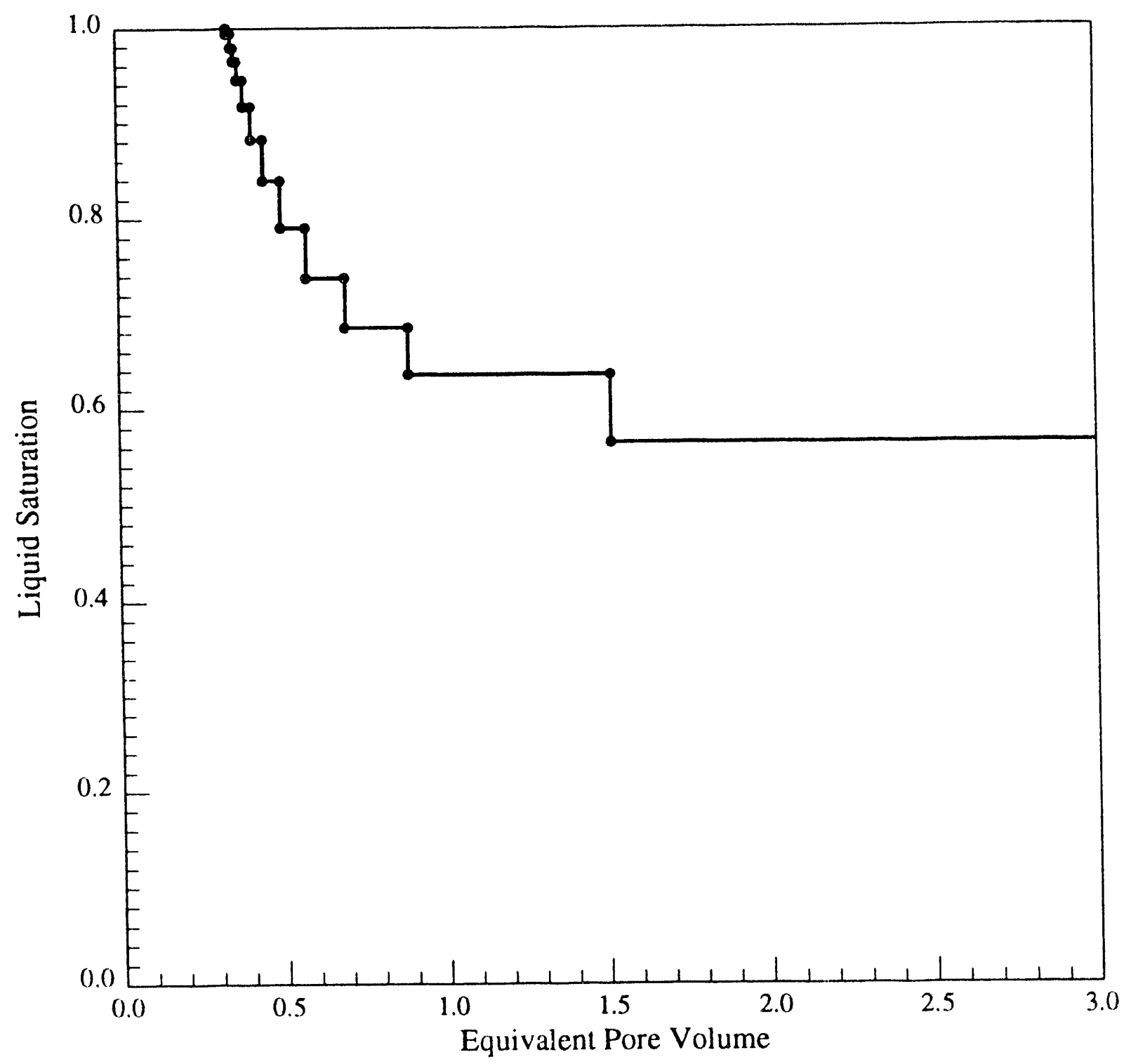

Figure 6.19: Calculated Saturation History for Foam of $0.1 \%$ Surfactant Concentration 


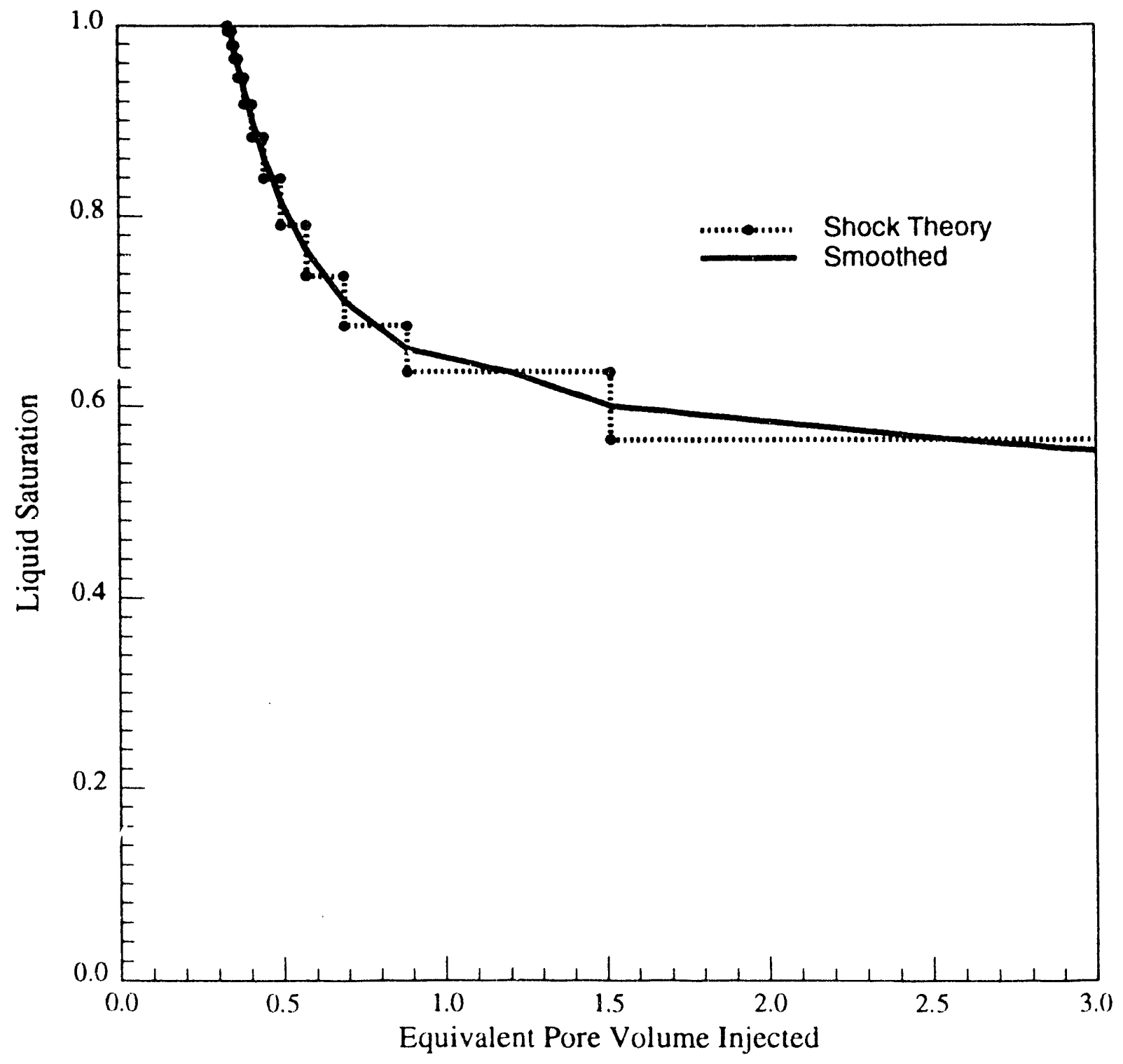

Figure 6.20: Calculated and Smoothed Saturation Histories for Foam of $0.1 \%$ Surfactant Concentration 


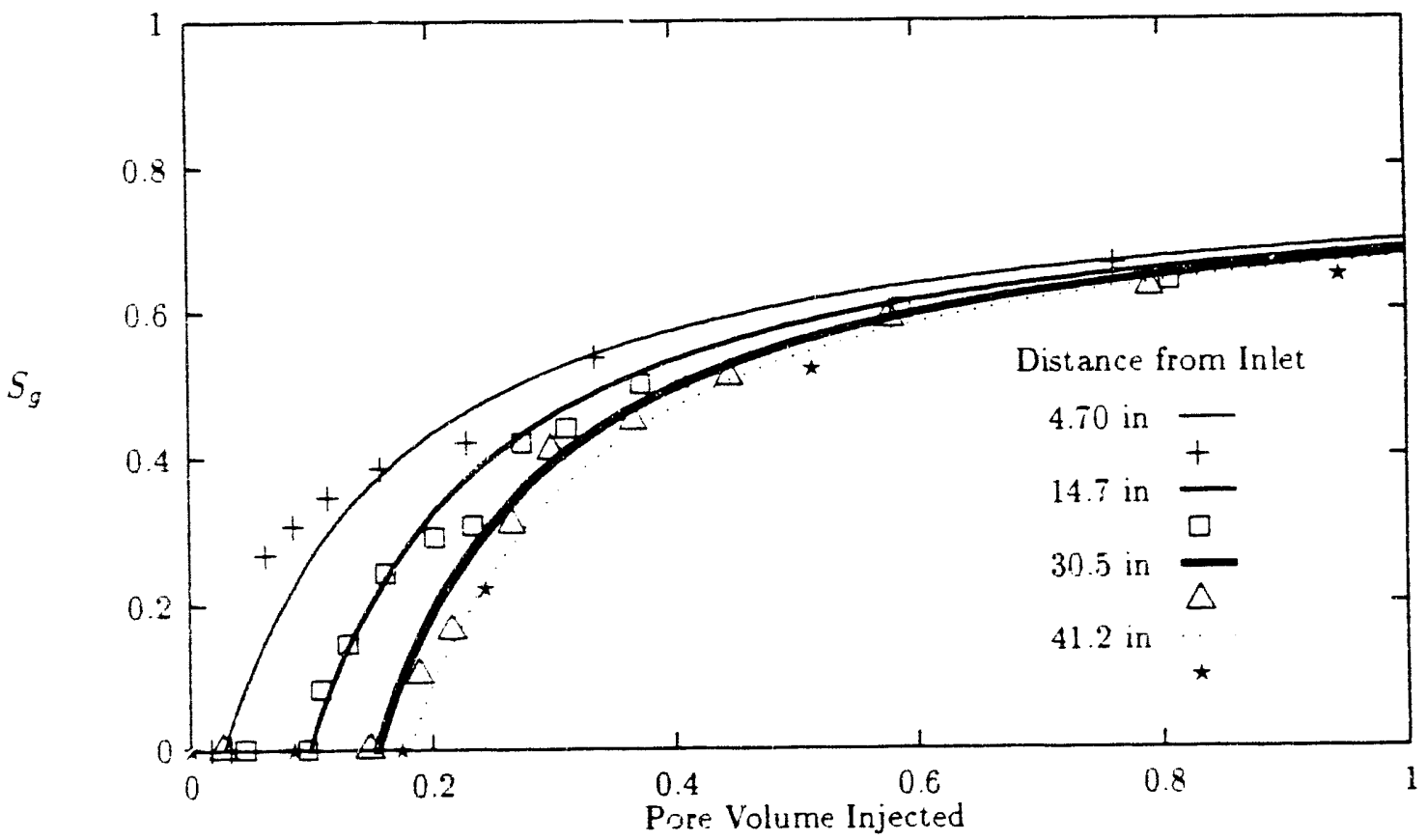

Figure 6.21: Saturation History Fits for Run 42 (0.1\% Surfactant Concentration) 


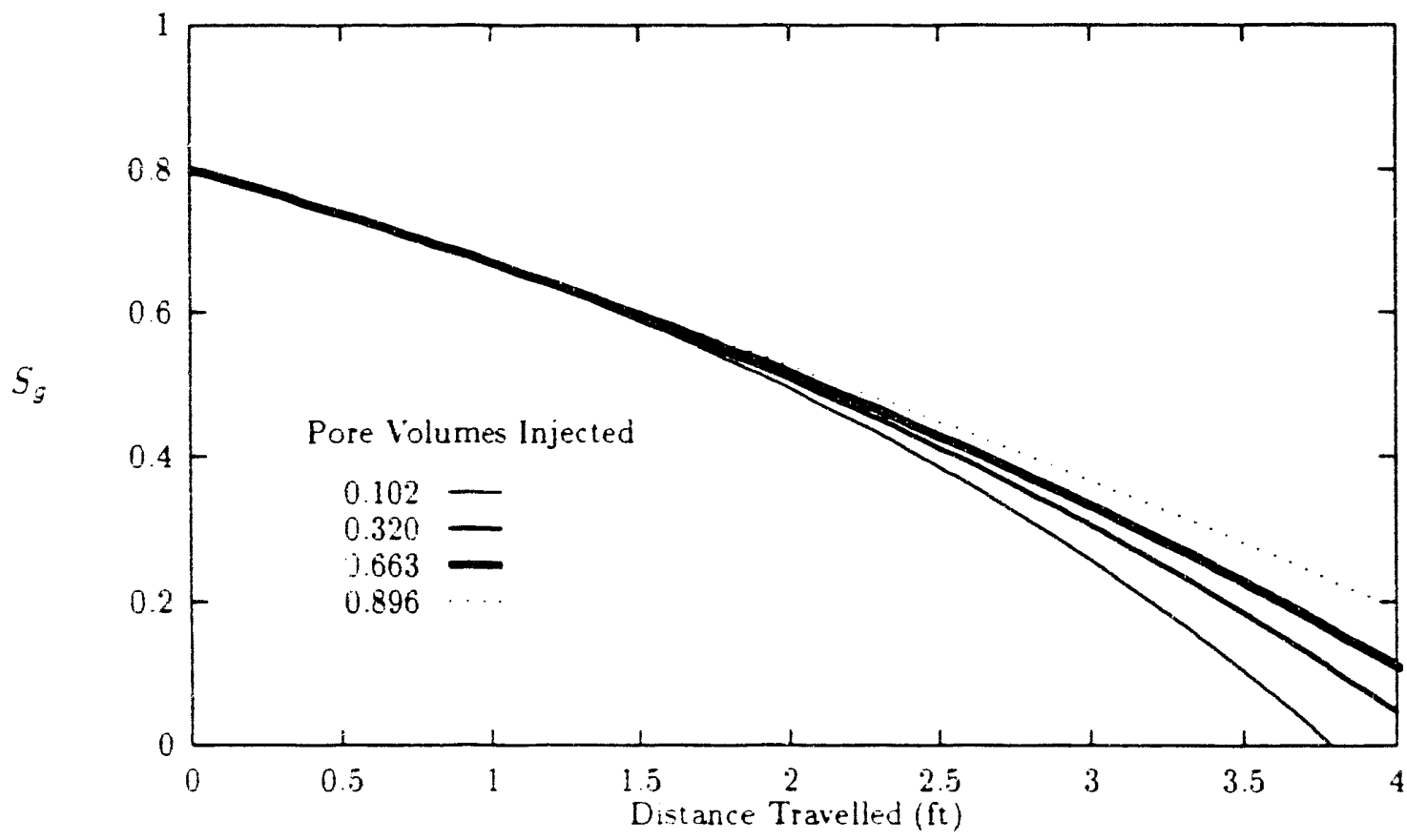

Figure 6.22: Saturation Profiles for Run 42 (0.1\% Surfactant Concentration) 


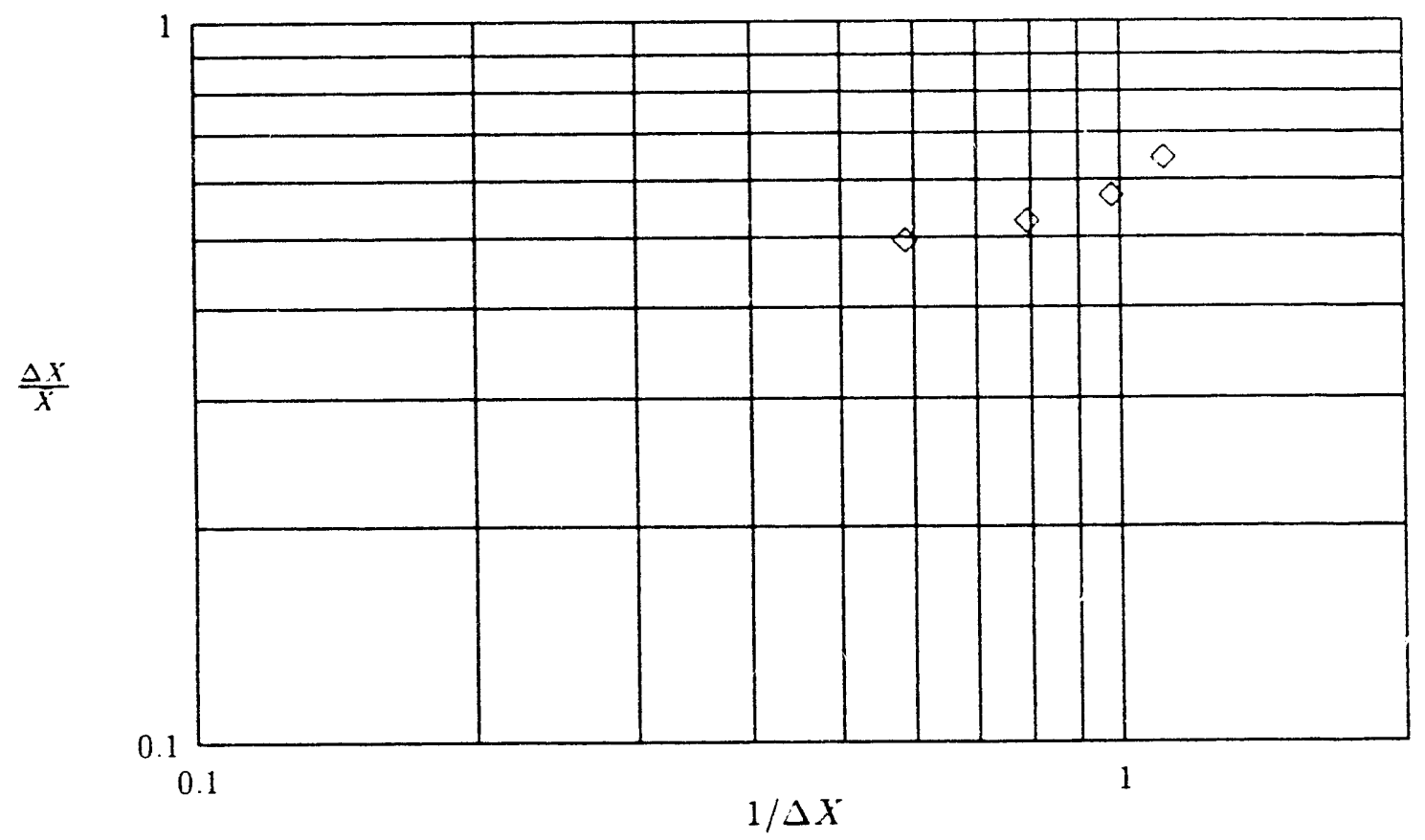

Figure 6.23: Type Curve Matching Data for Run 42

These data were then plotted in Figure 6.23. A least squares fit to Equation 4.17 gave a $C$ of 0.295 and $\alpha$ of 0.140 , which were the same as in Run 41 for practical purposes. Again. it can be seen that the mixing effect, though important, did not play a dominating role in the run. 
Having found the mixing coefficients, they were applied to the final match of the experimental data. The final match of Run 42 is shown in Figure 6.24. The match is also good. The final matches of these runs are especially good, considering the simplicity of the theory.

\subsubsection{Matching of Recovery}

The recovery data from the experiments were, unfortunately, lost. Since the in-situ saturation measurements from the CT scans appear to be reliable, it was decided to salvage the recovery data from them.

As we have already obtained the saturation profiles for four different times (Figures 6.15 and 6.22), the average saturations in the sandpack were estimated by in tegrating the saturation curve over the length of the sandpack. Since the initial gas saturation was zero, the recovery factor is equal to the average gas saturation in the sandpack.

The recovery matches for Runs 41 and 42 are shown in Figures 6.25 and 6.26. The matches are good. The calculated recovery curves are not smooth as a result of the shocks used in the calculations. However, the fact that these matches were so close to the experimental data is important, for this is the ultimate goal of the theory. It is clear from these results that the theory works well for these two cases. Of course it should be tested for many other saturations and variable ranges. From these two figures, it can also be seen that the recovery factor for the higher concentration is slightly higher than that for the lower concentration.

\subsubsection{Matching of Pressure Drops}

The pressure drop at any time could be calculated by summing up the pressure drops that are associated with the various shocks. At each different time, the mixing effect had to be taken in to account for the saturation profile. The saturation profiles could then have been calculated. Once these were calculated, the individual pressure calculations could have been made over each shock segment. These could then be added to predict the overall pressure drop as a function of time. This would have been a tedious series of calculations, with little hope for useful results, for the pressure data for the constant concentration runs of Chapter 5 were poorly matched by the calculations. So these calculations were not atternpted, however, the procedures that would be required are outlined in Appendix $F$. 


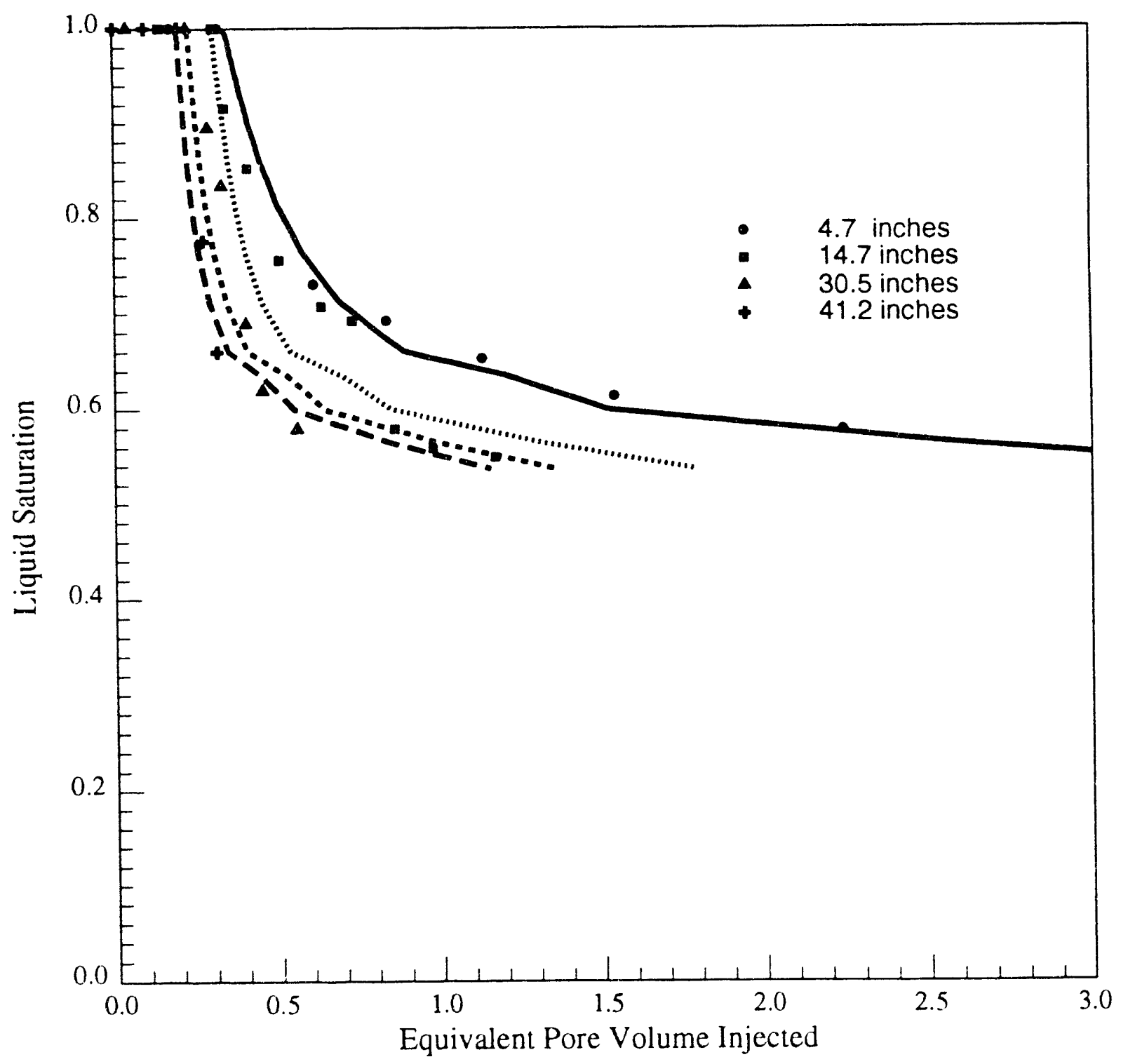

Figure 6.24: Final Saturation History Match for Run 42 (Flow of 0.1\% Surfactant Foam) 


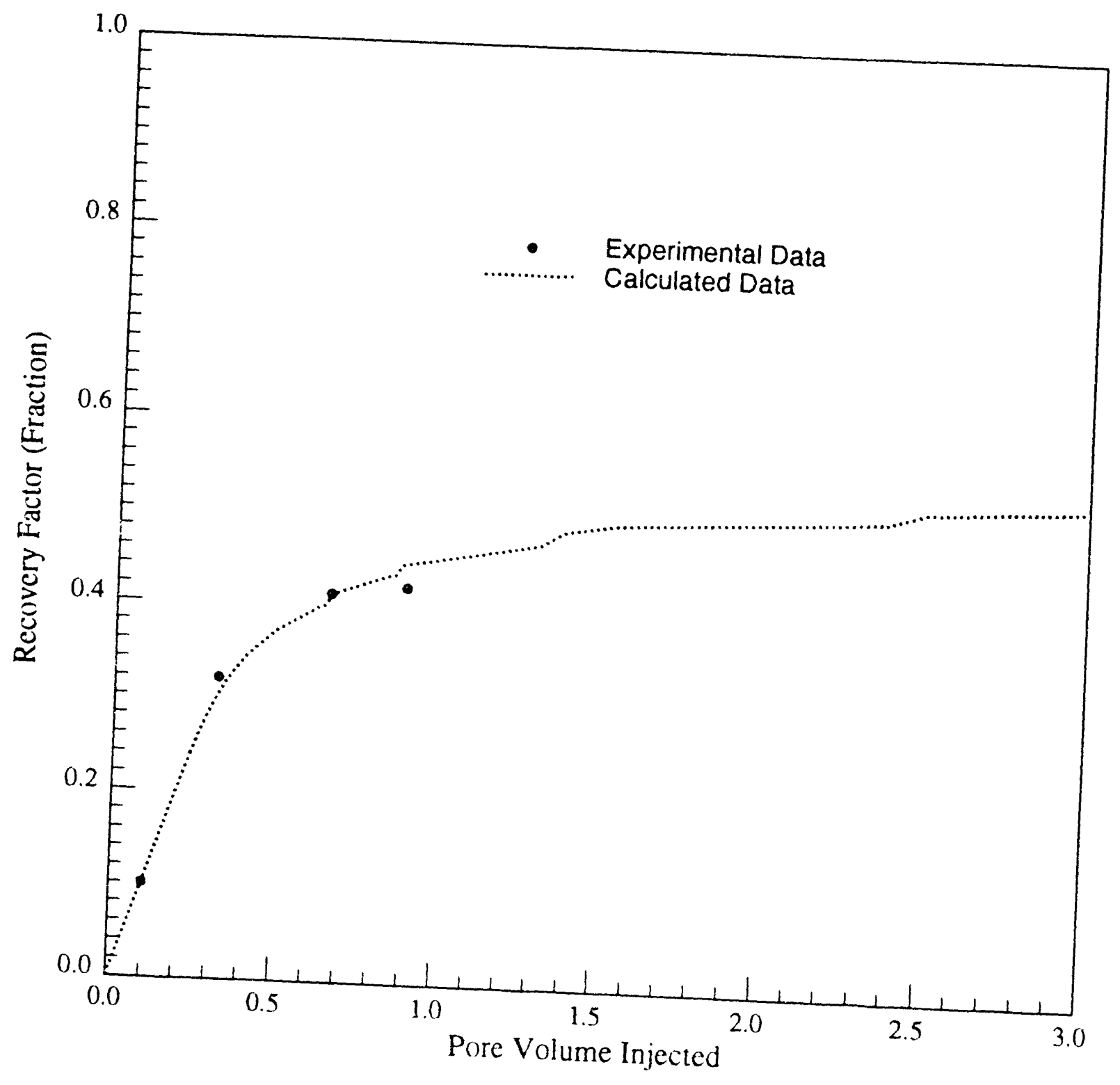

Figure 6.25: Recovery Match for Run 41 (Flow of 1.0\% Surfactant Foam) 


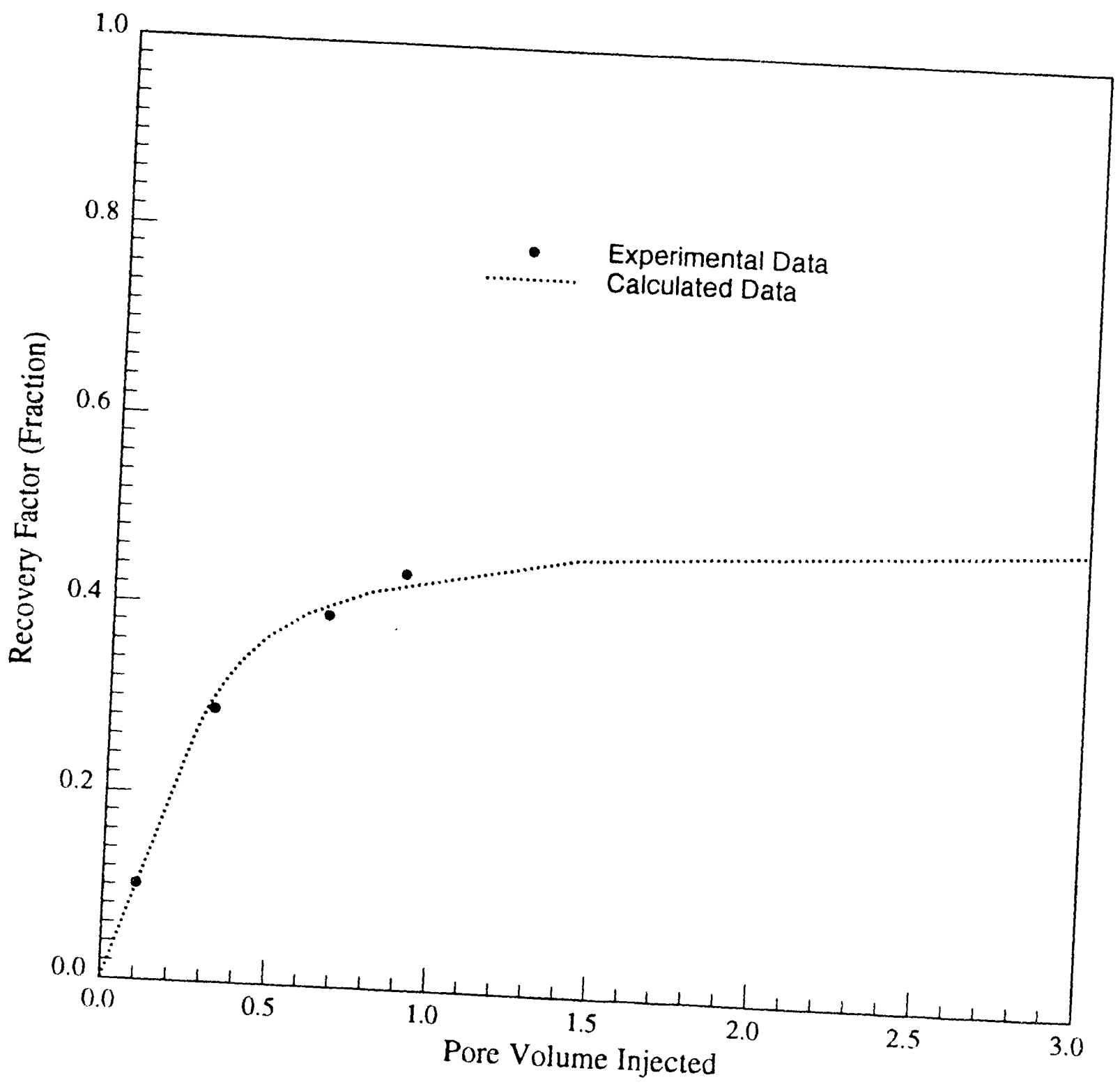

Figure 6.26: Recovery Match for Rin 42 (Flow of $0.1 \%$ Surfactant Foam) 


\subsection{Some Implications of the Results}

The theory proposed in this work enables us to better understand the foam flow mechanism. Foam flow does not follow Buckley-Leverett theory. It can be thought of as being romposed of infinitesimal slugs of gas, with the gas front having the lowest apparent viscosity and the injected foam having the highest possible apparent viscosity. Each infinitesimal slug results in a shock during the displacement. The higher viscosities produce shocks that move more slowly. Thus it takes many pore volumes of foam injection for the displacement to reach the residual state.

The surfactant adsorption has some effect on the foam flow behavior. It retards the foam front velocity in the low concentration range. However, the experimental results and the calculations show that the effect is not very significant for the bulk of the displacement.

Mixing that takes place during foam flow distinguishes the foam displacement from Buckley-Leverett types. So to predict the foam behavior downstream, an appropriate theory has to take this in to consideration.

Having discussed the validity of the new proposed theory in predicting the experiments, the author also recognized the fact that these experiments were made under some limitations as mentioned before. This theory may indeed predict, reasonably well, displacements under other conditions such as different flow rates or foam qualities, but more experiments are needed to verify the validity of this approach.

From all the experimental runs, it can be seen that the displacement is more efficient for pure gas displacements of surfactant-laden water than for foam displacements at the same surfactant concentration. This brings about an interesting point that it may be better to generate the foam in situ than injecting foam. That is, it seems clear that the surfactant should be placed ahead of the gas to the extent possible. However, the amount of surfactant. needed for pure gas displacements is much higher than that for foam displacements. So some eronomic calculations should be made before making a decision about how best to operate. 


\begin{tabular}{|r|r|}
\hline$\Delta X$ & $\bar{X}$ \\
\hline 0.857 & 1.325 \\
\hline 0.975 & 1.706 \\
\hline 1.201 & 2.328 \\
\hline 1.625 & 3.320 \\
\hline
\end{tabular}

Table 6.2: $\Delta X$ and $\bar{X}$ Values for Run 41

\begin{tabular}{|c|r|}
\hline$\Delta X$ & $X$ \\
\hline 0.900 & 1.391 \\
\hline 1.024 & 1.791 \\
\hline 1.261 & 2.394 \\
\hline 1.706 & 3.446 \\
\hline
\end{tabular}

Table 6.3: $\Delta X$ and $\bar{X}$ Values for Run 42 


\section{Chapter 7}

\section{Conclusions}

The following conclusions can be made from the experimental, theoretical, and numerical in vestigations:

- Foam flow is not a piston-like process. Nor does it follow the Buckley-Leverett theory.

- Gas channeling and fingering take place at the foam front, leaving much of the in-situ fluid untouched.

- It takes many pore volumes of foam injection to achieve the residual condition, i.e. the initial displacement process is slow.

- Surfactant adsorption at the foam front plays only a minor role in the process for the surfactant and porous medium studied here.

- Mixing in the foam front is also an important factor in foam flow that distinguishes the process from the Buckley-Leverett theory.

- A new and simple theory has been proposed based on the fractional flow curve construction method plus mixing theory to describe transient foam flow in a porous medium. The theory matched the experimental data satisfactorily.

- Fractional flow curves can be determined from matching the experimental data of gas displacements of surfactant-laden solutions.

- The breakthrough time, final gas saturation, and apparent viscosity of foam can all be correlated to the surfactant concentration and be used for the prediction of foam flow.

- For the same surfactant concentration, pure gas displacement is more efficient than foam displacement. Some economics should be run to determine the best way to operate. 


\begin{tabular}{|r|r|}
\hline$\Delta X$ & $\bar{X}$ \\
\hline 0.857 & 1.325 \\
\hline 0.975 & 1.706 \\
\hline 1.201 & 2.328 \\
\hline 1.625 & 3.320 \\
\hline
\end{tabular}

Table 6.2: $\Delta X$ and $\bar{X}$ Values for Run 41

\begin{tabular}{|r|r|}
\hline$\Delta X$ & $X$ \\
\hline 0.900 & 1.391 \\
\hline 1.024 & 1.791 \\
\hline 1.261 & 2.394 \\
\hline 1.706 & 3.446 \\
\hline
\end{tabular}

Table 6.3: $\Delta X$ and $\bar{X}$ Values for Run 42 


\section{Chapter 7}

\section{Conclusions}

The following conclusions can be made from the experimental, theoretical, and numerical investigations:

- Foam flow is not a piston-like process. Nor does it follow the Buckley-Leverett theory.

- Gas channeling and fingering take place at the foam front, leaving much of the in-situ fluid untouched.

- It takes many pore volumes of foam injection to achieve the residual condition, i.e. the initial displacement process is slow.

- Surfactant adsorption at the foam front plays only a minor role in the process for the surfactant and porous medium studied here.

- Mixing in the foam front is also an important factor in foam flow that distinguishes the process from the Buckley-Leverett theory.

- A new and simple theory has been proposed based on the fractional flow curve construction method plus mixing theory to describe transient foam flow in a porous medium. The theory matched the experimental data satisfactorily.

- Fractional flow curves can be determined from matching the experimental data of gas displacements of surfactant-laden solutions.

- The breakthrough time, final gas saturation, and apparent viscosity of foan can all be correlated to the surfactant concentration and be used for the prediction of foam flow.

- For the same surfactant concentration. pure gas displacement is more efficient than foam displacement. Some economics should be run to determine the best way to operate. 
- The pressure gradient behind the foam front is much greater than that in front of the foam.

- The apparent viscosity of foam can become very high at high surfactant concentrations. It is certainly much greater than either of its components: gas or water. 


\section{Chapter 8}

\section{Recommendations for Future Work}

Although this work has proposed a new theory that appears to work, there is still much work to be done to improve the theory to be used in real field applications. The following recommendations for the future are made.

- CATPIX (a software package from SHELL) should be used to process the CT pictures of this work on a pixel by pixel basis to understand the variability in saturations seen in this type of displacement.

- More experiments need to be done at broader concentration ranges than studied here. Also various surfactants should be used to see if they behave in a similar manner.

- The injection rate should be tested as another experimental variable.

- Various gas/surfactant injection ratios (foam quality) should be tested.

- The effect of residual oil should be investigated, as well as other porous medium types.

- The theory should be extended to two and three dimensions. 


\section{Chapter 9}

\section{Nomenclature}

English letters

$a$ - a constant coefficient, Langtnuir constant

$b$ - a constant coefficient, Langmuir constant

$f_{S}$ - fractional flow of gas

$f_{30}$ - gas fractional flow at initial condition

$f_{g_{1}}$ - gas fractional flow at first shock

$f_{g 1}^{\prime}$ - tangent of first fractional flow curve

$f_{y_{2}}$ - gas fractional flow at second shock

$f_{g_{2}}^{\prime}$ - tangent of second fractional flow curve

$f_{g_{n}}$ - jas fractional fiow at $n$th shock

$f^{\prime}{ }^{n}$ - tangent of $n$th fractional flow curve

$f_{g_{n-1}}$ - gas fractional flow at (n-1)th shock

$k$ - absolute permeability, darcies

$k_{r g}$ - relative permeability of gas

$k_{r g}^{0}$ - end-point relative permeability of gas

$k_{\tau w}$ - relative permeability of water

$k_{r w}^{0}$ - end-point relative permeability of water

$n_{g}$ - relative permeability index for gas

$n_{w}$ - relative permeability index for water

qfoam - foam flow rate, $\mathrm{cc} / \mathrm{min}$

$q_{g}$ - gas flow rate, cc/min

$q_{g s:}$ - gas flow rate at standard conditions, cc/min

$q_{i}$-- liquid flow rate, $\mathrm{cc} / \mathrm{min}$

$t_{B T}$ - breakthrough time, PV

to - dimensionless time, pore volumes

$x$ - linear distance, $\mathrm{ft}$ or $\mathrm{cm}$

$x$ - a variable

$A$ - cross-sectional area. $c^{m}{ }^{2}$

$\therefore \quad$ - activity of surfactan ${ }^{+}$fraction

- surfartani concentration, wot 


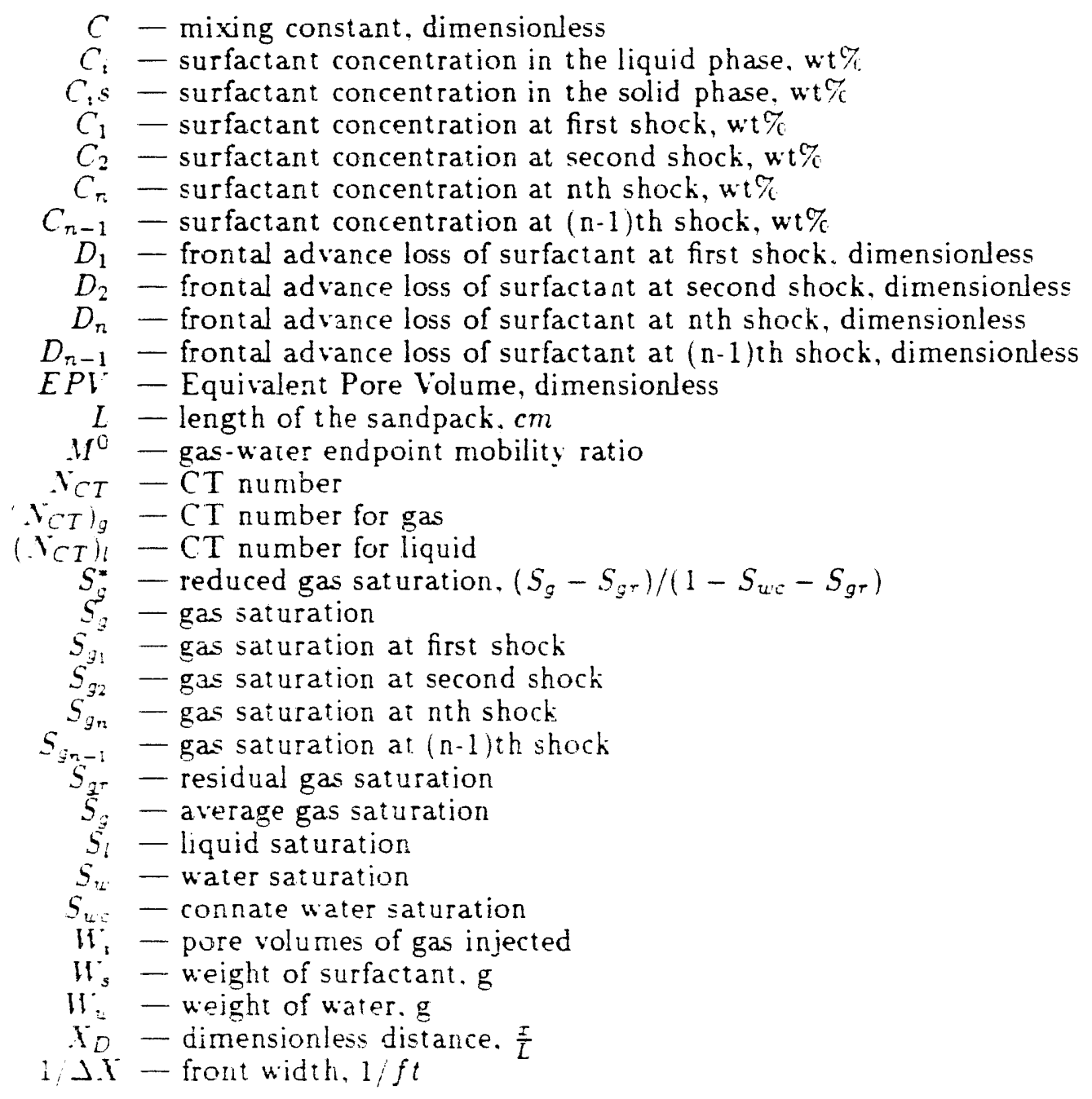

Greok letters

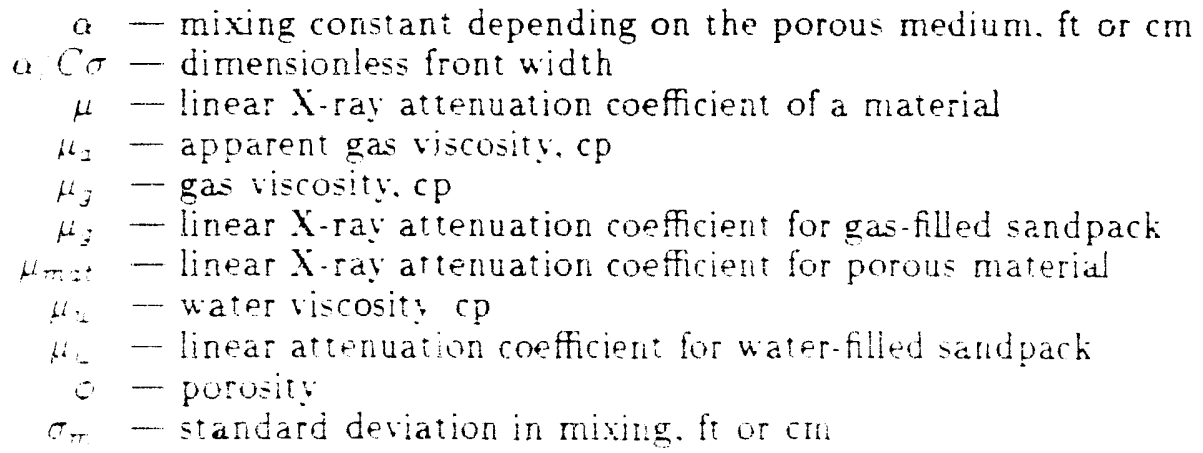


$\sigma_{B L}$ - standard deviation in Buckley-Leverett theory. $\mathrm{ft}$ or $\mathrm{cm}$

$\sigma / C x$ - dimensionless front location

$v_{1}$ - first gas shock velocity, dimensionless

$v_{2}$ - second gas shock velocity, dimensionless

$v_{n}$ - nth gas shock velocity, dimensionless

$\Delta P$ - pressure drop, atm or psi

$\Delta X / \ddot{X}$ - front location, dimensionless

$\Gamma$ - foam quality 


\section{Bibliography}

[1] Al-Khafaji, A. A., Castanier, L. M., and Brigham, W. E.: "Effect of Temperature on Degradation Adsorption And Phase Partitioning of Surfactants Used in Steam Injection for Oil Recovery," No. SUPRI-TR-38, Stanford U. Petroleum Research Inst., Stanford, CA (Dec. 1982).

[2] Ault, J. W., Johnson, W. M., and Kamilos, G. N.: "Conversion of Steamfloods to Low Quality Steam and/or Hot Water Injection Projects," paper SPE 13604 presented at the 198555 th California Regional Meeting, Bakersfield, CA, March 27-29.

[3] Bernard, G. G., Holm, L., and Jacobs, W.: "Effect of Foam on Trapped Gas Saturation and on Permeability of Porous Media to Water," Soc. Pet. Eng. J. (Dec. 1965) 295-300.

[1] Bond, D. C. and Holbrook, O. C.: "Gas Drive Oil Recovery Process," U.S. Patent No. $2,866.507$ (1958).

[5] Brigham, W. E.: "Mixing Equations in Short Laboratory Cores," Soc. Pet. Eng. J. (February 19i4) 91-99.

[6] Brigham, W. E.: "Mixing Equations in Various Geometries," SPE Reservoir Engineering (March 1986).

[i] Brigham, W. E.: "PE270A Advanced Reservoir Engineering Handouts," Stanford University (1990).

[8] Brigham. W. E.. Ramey, H. J. J., and Castanier, L. M.: "SUPRI Heavy Oil Research Program - Thirteenth Annual Report," No. SUPRI-TR-76, Stanford U. Petroleum Research Inst., Stantord, CA (August 1989).

[9] Brigham. W. E.. Reed, P. W.. and Dew, J. N.: "Experiments on Mixing During Miscible Displacement in Posous Media," Soc. Pet. Eng. J. (March 1961) 1 \%.

[10] Brooker. 11. J.: Computed Tomography for Radicaraphers, MTP Press Limited (19x(0).

[11] Castanier. L. M.: "Steam with Additives: Field Projects of the Fighties." Journal of Petroleum Scifserf and Enginetring (19×9) No. 2. 
112: Casteel, J. F. and Djabbarah, N. F.: "Sweep Improvement in $\mathrm{CO}_{2}$ Flooding by lise of Foaming Agents." SPE Reservoir Engintering (November 1988).

[13: Chang. S.-H., Owusu, L. A., French. S. B., and Fovarik, F. S.: "The Effect of Microscopic Heterogeneity on $\mathrm{CO}_{2}$-Foam Mobility: Part 2-Mechanistic Foam Simulation," paper SPE 20191 presented at the 1990 SPE/DOE Seventh Symposium on Enhanced Oil Recovery, Tulsa, OK, April 22-25.

[14] Chiang. J. C., Sanyal, S. K., Castanier, L. M., Brigham, W. E.. and Sufi, A.: "Foam as a Mobility Control Agent in Steam Injection Processes," paper SPE 8912 presented at the 198050 th Annual California Regional Meeting. Los Angeles, A pril 9-11.

[15) Demiral. M. R. B. and Okandan. E.: "Experimental Analysis of Steam Foam Injection to Heary Oil Limestone Reservoirs," paper SI'E $1573+$ presented at the 1987 Fifth SPE Middle East Oil Show, Manama. Bahrain, March $i-10$.

[16] Dilgren, R. E., Deemer, A. R., and Owens, K. B.: "The Laboratory Development and Field Testing of Steam/noncondensible Gas Foams for Mobility. Control in Heavy Oil Recovery:" paper SPE $107 i 4$ presented at the 1982 52nd Annual California Regional Meeting. San Francisco, March 24-2.5.

[17] Dornan. R. G.: "Hot Waterflood in a Post Steamflood Reservoir: A Case History in the Kern River Field, California," paper SPE 20050 presented at the 199060 th California Regional Meeting of SPE. Ventura, CA, April 4-6.

[18) Doscher. T. M., Kruskaa, V. A., and Hammershaib, E. C.: "Analysis of Five Field Tests of Steam Drive Additives," paper SPE 12057 presented at the 198358 th Annual California Regional Meeting. San Francisco. April 4-6.

[19) Ettinger. R. A. and Radke. C. J.: "The Influence of Texture on Steady Foam Flow in Berea," paper SPE 19688 presented at the 198964 th Annual Technical Conference and Exhibition, San Antonio, TX. Oct. 8-11.

[20] Falls. A. H.. Hirasaki, G. J., Patzek, T. W.. Gauglitz, D. A., Miller. D. D., and Ratulowski, T.: "Development of a Mechanistic Foam Simulator: The Population Balance and Generation by Snap-Off," SPE Reservoir Enginetring (August 1988).

[21] Falls. A. H. Musters, J.. and Ratulowski. J.: "The Apparent Viscosity of Foams in Homogeneous Bead Packs," SPE Reservoir Engintering (May 1989) 15.5-16.4.

[22] Fisher. A. W., Foulser. R. W. S., and Goodyear, S. G.: "Mathematical Modelling of Foam Flooding." paper SPE 20195 presented at the 1990 SPE/IOOE Seventh Symposium on Enhanced Oil Rocovery. Tulsa. OK. April 22.25.

23. Frifed. A. X.: "The Foam Drive Proces for Increasing the Recovery of Oil." No. Roport

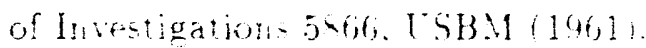


[24] Friedmann, F., Chen. W. H.. and Cauglitz, P. A.: "Experimental and Simulation Study of High-Temperature Foam Displacement in Porous Media," SPF Reservoir Enginecring (February 1991).

[25] Friedmann, F., Smith, M. E., Guice, W. R., Gump, J. M., and Nelson, D. G.: "Steam Foam Mechanistic Field Trial in the Midway-Sunset Field," paper SPE 21780 presented at the 1991 60th California Regional Meeting of SPE, Long Beach, CA, March 20-22.

[26] Helfferich. F. (i.: "Theory of Multicomponent. Multiphase Displancement in Porous Media," Soc. of Pet. Eng. J. (February 1981).

[27] Helfferich, F. G.: "Generalized Welge Construction for Two-Phase Flow in Porous media in System with Limited Miscibility," paper SPE 16709 presented at the 1982 $5 i t h$ Annual Fall Technical Conference and Exhibition of the Society of Petroleum Engineering. New Orleans. LA. September 26-29.

[28] Hirasati. G. T. and Lawson, J. B.: "Mechanism of Foam Flow In Porous Media -Apparent Viscosity in Smooth Capillaries," paper SPE 12129 presented at the 1983 5 sth Annual Techincal Conference and Exhibition, San Francisco, Oct. 5-8.

[29] Hirasaki, J. G.: "The Steam Foam Process," J. of Pet. Tech. (May 1989).

[30] Holbrook, S. T., Patton. J. T., and Hsu, W.: "Rheology of Míobility Control Foams," paper SPE 9809 presented at the 1981 SPE/DOE Second Symposium on Enhanced Oil Recovery. Tulsa. OK, A pril 5-8.

[31] Holm. L. W.: "The Mechanism of Gas and Liquid Flow Through Porous Media in the Presence of Foam." Soc. Pet. Eng. J. (Dec. 196×) 359-369.

[32] Holm. L. W' and Carrison, W. H.: " $\mathrm{C}^{\prime} \mathrm{O}_{2}$ Diversion Using Foam in an Immiscible $\left(\mathrm{C}_{2}\right.$ Field Project," SPE Reservoir Enginecring (Feb. 1968) 112-118.

[33] Hong. K. C.: "Guidelines for Converting Steamflood to Waterflood," SPF. Restroir Enginetring (February 1987).

[34] Hong. K. C.: "Water Alternating Steam Process Improves Projeci Economics at Went Coalinga Field," (TM/SPE (90-84 1989).

[35] Hove. A. O.. Nilsen. V., and Leknes. J.: "Visualization of Xanthan Flood Behavior in Core Samples by Means of X-Ray Tomography," SPE Restrvoir Enginecring (Nov. $1990)+45-450$

[36; Hove. A. O.. Ringen. J. K., and Read. P. A.: "Visualization of Laboratory Corefloods with the Aid of Computerized Tomography of X-rays," SPE Reservoir Engintering (Nat 1957) ]4R 154. 
[37] Hudgins, D. A. and Chung, F. T.-H.: "Long-Distance Propagation of Foams," paper SPE 20196 presented at the 1990 SPE/DOE Seventh Symposium on Enhanced Oil Recovery, Tulsa, OK, April 22-25.

[38] Huh, D. G., Cochrane, T., and Kovarik, F.: "The Effect of Microscopic Heterogeneity on $\mathrm{CO}_{2}$-Foam Mobility: Part 1-Mechanistic Study," J. of Pet. Tech. (Aug. 198?) $872-879$.

[39] Hunt, P. K., Engler, P., and Bajsarowicz, C.: "Computed Tomography as A Core Analysis Tool: Applications, Instrument Evaluation, and Image Improvement." J. of Pet. Tech. (September 1988).

[40] Khatib, Z. I., Hirasaki, G. J., and Falls, A. H.: "Effects of (apillary Pressure on Coalescence and Phase Mobilities in Foams Flowing Through Porous Media," paper SPE 15442 presented at the 1986 61st Annual Technical Conference and Exhibition. New Orleans, Oct. 5-8.

[41] Lake, L. W.: Enhanced Oil Recovery, Englewood Cliffs, N.J. Prentice Hall (1989).

[42] Langmuir, I.: "Theory of Adsorption," Phys. Rev. (January 1915).

[43] Lau, H. C. and O'Brien, S. M.: "Surfactant Transport Through Porous Media in Steam-Foam Processes," SPE Reservoir Engineering (November 1988).

[44] Lee, H. O., Heller, J. P., and Hoefer, A. M. W.: "Change in Apparent Viscosity of $\mathrm{CO}_{2}$ Foam With Rock Permeability," paper SPE 20194 presented at the 1990 SPE/DOE Seventh Symposium on Enhanced Oil Recovery, Tulsa, OK, April 22-25.

[45] Lescure, B. M. and Claridge, E. L.: " $\mathrm{CO}_{2}$ Foam Flooding Performance vs. Rock Wettability." paper SPE 15445 presented at the 198661 st Annual Technical Conference and Exhibition, New Orleans, Oct. 5-8.

[46] Liu, D. B.: "The Accessibility of Residual Oil by The Oil Bank," Master's thesis. University of Waterloo, Waterloo, On, Canada (1986).

[47] Liu, I). B., Castanier, L. M., and Brigham, W. E.: "Analysis of Transient Foam Flow in 1-D Porous Media with CT," paper SPE 20071 presented at the 1990 60th California Regional Meeting of SPE, Ventura, CA, April 4- 6 .

[48] Mahmood, S. M. and Brigham, W. E.: "Two Dimensional Displacement of Oil by (ian and Surfactant Solution under Foaming Conditions," No. SEPRJ-TR-5s. Stanford l". Petrolfum Research Inst., Stanford, CA (Jul. 1987).

[49] Marfoe, C. H., Kazemi, H., and Ramirez, W. F.: "Numerical Simulation of Foam Flow in Porous Media," paper SPle 16709 presented at the $198 \pi$ 62nd Annual Torh. nical conference and Exhibition of the Socieng of Petroleum Enginering. I)allas. TX. Sipprimber 2830 . 
[50] Marsden, S. S.: "Foans in Porous Media," No. US DOE Report No. DE-AC0:381SF1156.1, Stanford U. Petroleum Research Inst., Stanford, ('A (May 19x6).

[51] Marsden, S. S.. Elson, T., and Guppy, K.: "Literature Review of the Selective Blockage of Fluids in Thermal Recovery Projects," No. SUPRI-TR-3, Stanford U. Petroleum Research Inst., Stanford, CA (Dec. 1977).

[52] Marsden. S. S. and Khan, S. A.: "The Flow of Foam Through Short Porous Media and Apparent Viscosity Measurements," S'oc. Pet. Eng. J. (March 1966) 17-25.

[53] Minssienx. L.: "Oil Displarement by Foams in Relation to Their Physical Properties in Purous Media," J. of Pet. Tech. (Jan. 1974) 100 10x.

[54] Mitchell, B. J.: V'iscosity of Foam, Ph1) dissertation, U. of Oklahoma, Norman (1969).

[55] Mohammadi, S. S. and Tenzer. J. R.: "Steam-Foam Pilot Project at Dome-Tumbador, Midway Sunset Field: Part 2," paper SPE 20201 presented at the 1990 SPE/DOE Seventh Symposium on Enhanced Oil Recovery, Tulsa, OK, April 22.25.

[56] Morgan, C. L.: Basic Principles of Computed Tomography, Baltimore Md: Iniversity Park Press (1983).

[5i] Nikolov, A. D., Wasan, D. T., Huang. D. W., and Edwards, D. A.: "The Effect of Oil on Foam Stability, Mechanisms and Inplications for Oil Displacement by Foam in Porous Media." paper SPE 15443 presented at the 198661 st Annual Technical Conference anc. Exhibition, New Orleans, Oct $5-8$.

[58] Peters, E. J. and Hardham, W. D.: "Visualization of Fluid Displacements in Porous Media lising Computed Tomography Imaging," Journal of P'etroleum Sicience and Enginetring (April 1990).

[59] Radke, C. J. and Ransohoff, 'T. C.: "Mechanisms of Foam Generation in Cilass Bead Packs," paper SPE 15441 presented at the 198661 st Annual Technical (onference and Exhibition. New Orleans, Oct. 5-8.

[60] Ransohoff, T. C $\therefore$ and Radke, C. J.: "Mechanism of Foan Generation in Cilass-Bead Packs." SPE Rescruoir Engintering (May 1988).

[6il] Raza. S. H. and Marsden, S. S.: "The Streaming Potential and Rheology of Foam," Soc. Pet. Eng. J. (1)ec. 196i) 359-36rs.

[62] Rohin. M.: "Laboratory Work on Fuaming Additives to Improve Steim Drive Ef. ficiency." presented at the 198.5 3rd European Meeting on Improved Oil Recovery, Roma. Italy, A pril 16-18.

[0.3] Rossen, W. R.: "Theories of Foam Mobilization Pressure (iradient." paper SPE 1735K presented at the 1988 SPF/D)E Sixth Sympesium on Enhanced Oil Rorovery, Tulsa. Oh. April $17 \cdot 20$. 
[6.t] Sanchez, J. M. and Hazlett, R. D.: "Foam Flow Through an Oil- Wet Porous Medium: A Laboratory Study," paper SPE 1969 i presented at the 198964 th Annual Technical Conference and Exhibition, San Antonio. TX. Oct. 8-11.

[65] Sanchez, J. M. and Schechter, R. S.: "The Effect of Trace Quantities of Surfactant on Nitrogen/water Relative Permeabilities," paper SPE 15446 presented at the $198661 \mathrm{st}$ Annual Technical Conference and Exhibition, New Orleans. Oct. 5-8.

[66] Shen, C. 11:: "Laboratory Hot Waterfloods Prior To and Following Steamfloods." paper SPE 1875t presented at the 1989 59th California Regional Meeting of SPE. Bakersfield. CA, April 5-7.

(6i) Sufi. A. H.: "A Method of Alternating Steam and Water Injection for Recovering Heary Oils," paper SPE 20246 presented at the 1990 SPE/DOE Seventh Symposium on Enhanced Oil Recovery, Tulsa, OK, April 22-25.

[6.] Tomutsa. L., Doughty. D., Mahmood, S., Brinkmeyer, A., and Madden. M.: "Imaging Techniques Applied to The Study of Fluids in Porous Media." No. NIPER-45 (DE91002215). NIPER (January 1991).

[69] Treinen, R. J.: "Apparent Viscosity Measurements of Surfactant Foam in Porous Media," Master`s thesis, Stanford L'., Stanford, CA (198.5).

[70] Vinegar, H. J.: “X-Ray CT and NMR Imaging of Rocks," J. of Pet. Tech. (March $1986)$.

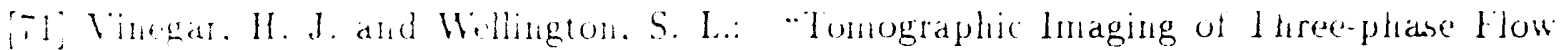
Experiments." Revieu of Scientific Inotrumentation (58, No.1) (January 19si).

[72] Mang. P. F., Al-Khafaji. A., and Castanier. I. M.: "Steam-Surfactant Sỵstems at Reservoir Conditions," No. Fossil Energy Report CONF 20712 . DOE (July 1982).

(73) Wang. S. Y.. Ayral, S., Castellana, F. S., and Gryte, C. C.: "Reconstruction of Oil Saturation Distribution Histories During Immiscible Liquid-Liquid Displacement by Computer-Assisted Tomography;" AIChEJ(July 198.1).

[i1) Wellington, S. L. and Vinegar, H. J.: "CT Studies of Surfactant-Induced $\mathrm{C}^{\circ} \mathrm{O}_{2}$ Mobility. Control," paper SPE 14393 presented at the 1985 60th Annual Technical Conference and Exhibition of the Socioty of Petroleum Engineering. Las Vegas, $\mathrm{NV}$, September $22-25$.

[75] Yang. C.-Z., Huang, Y.-H., and Han. D.-K.: "Andysis and Explanation to Industrial Pilot Foam-Flooding Results on the Lao Jun Miao Fëeld in China." paper SPE 17387 presented at the 19xx SPE/DOE Sixih Symponinn on Enhanced Oil Recovery. Talsa. ok. April 1:-20. 


\section{Appendix A}

\section{Titration Method}

The titration method is described in detail by Al-Khafaji ct al. (1982). The following is a brief description of the procedures and data pertinent to these particular experiments.

To obtain the surfactant concentration of a solution, the molarity is first calculated and then compared to the molarity of surfactant solutions of known concentrations. The molarity of a solution is obtained as follows:

$$
M_{S}=M_{H} \times V_{H} / V_{S}
$$

where $M_{S}$ is the molarity of the active sulfonate in the surfactant solution sample in mole/liter. $M_{H}$ is the molarity of the Hyamine 1622 solution in mole/liter, $H_{H}$ is the volume of $\mathrm{H}:$ amine 1622 solution used in $\mathrm{cm}^{3}$, and $\mathrm{l}_{\mathrm{s}}$ is the volume of surfactant sample taken in $\mathrm{cmi}^{3}$.

The molecular weight of surfactant AOS 1618 was 356 . So the molarity, $M_{S}$ at 1 wt $\%$ was calculated to be $0.02 \times 0$. The molarity of the Hyamine 1622 solution was 0.0013.th.

The actual titration procedures are an follows:

1. Add lis ml (usually 1 or $2 \mathrm{ml}$ in this work) of sample surfactail solution. $10 \mathrm{ml}$ of dis. tilled water. $15 \mathrm{ml}$ of chloroform and $10 \mathrm{ml}$ of acid indicator subtion 10 a heater. The acid indicator solution is a mixume of losulphine Blue $\mathrm{V}$ liso. Dindidum Bromide. Sulphuric acid solution, and dintilled water. 
2. Stopper the beaker and shake after each addition of titrant. 'I he solution shoukd hater on a light pink color at the bottom layer during the process and clears more quickly towards the end point.

3. The endpoint is reached when the pink color is completely gone and the bottom laver is a faint greyish blue. Note here that if the bottom laver becomes dark blue. you have gone too far. Another new sample must be used. So extreme care should be exercined during the process, especially when the total sample volume in very small.

4. Take note of the amount of titrant used, $V_{H}$.

Then $M_{S}$ was calculated from Equation A.1. Comparison of $M_{S}$ with the standard $M_{S}$ vajues at known surfactant concentrations ( $1 \%, 0.1 \%$, and $0.01 \%)$ gave rise to the surfactant concentration of the sample solution. 


\section{Appendix B}

\section{Data Acquisition}

Pressure data measurement and recording program:

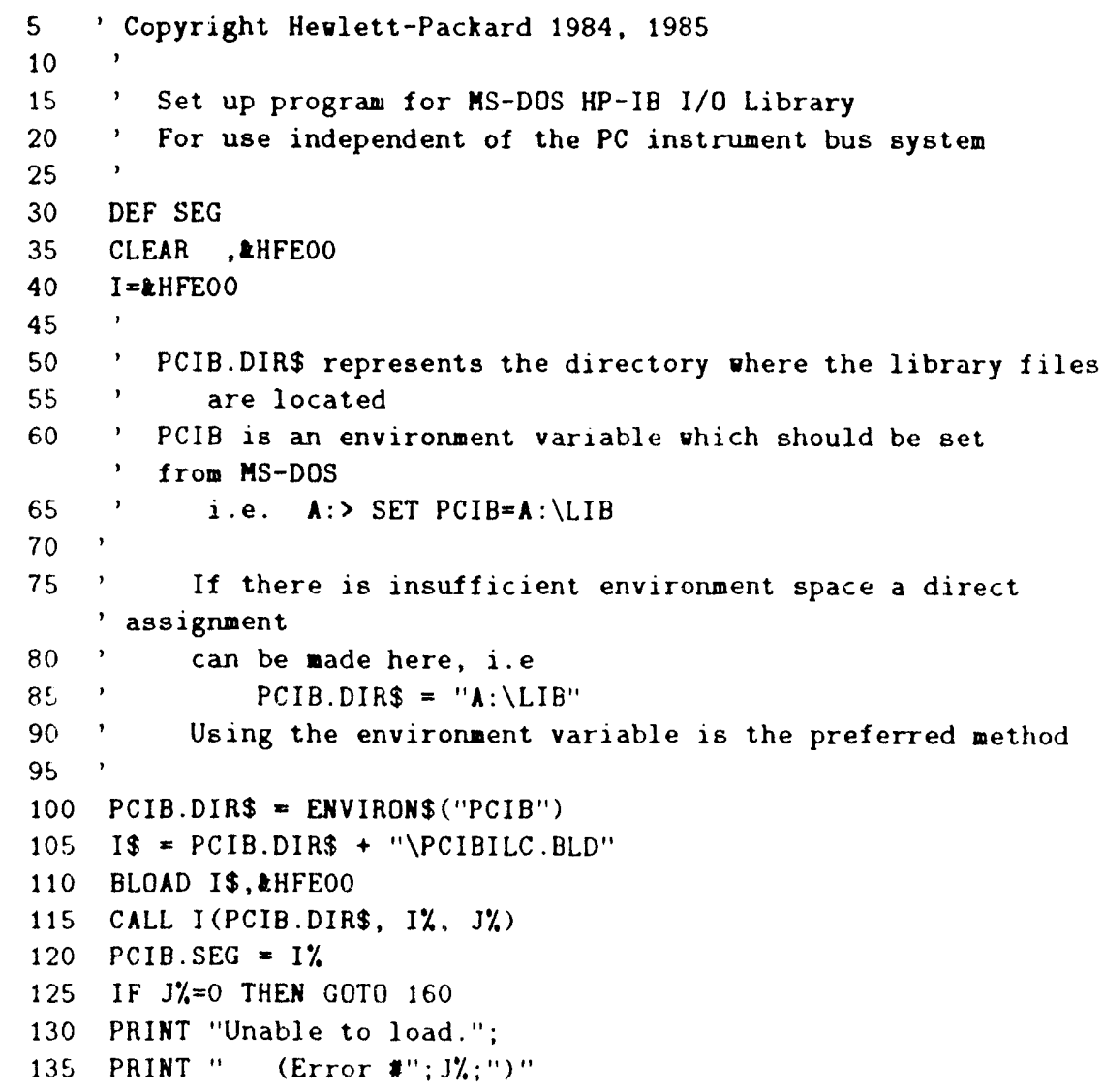




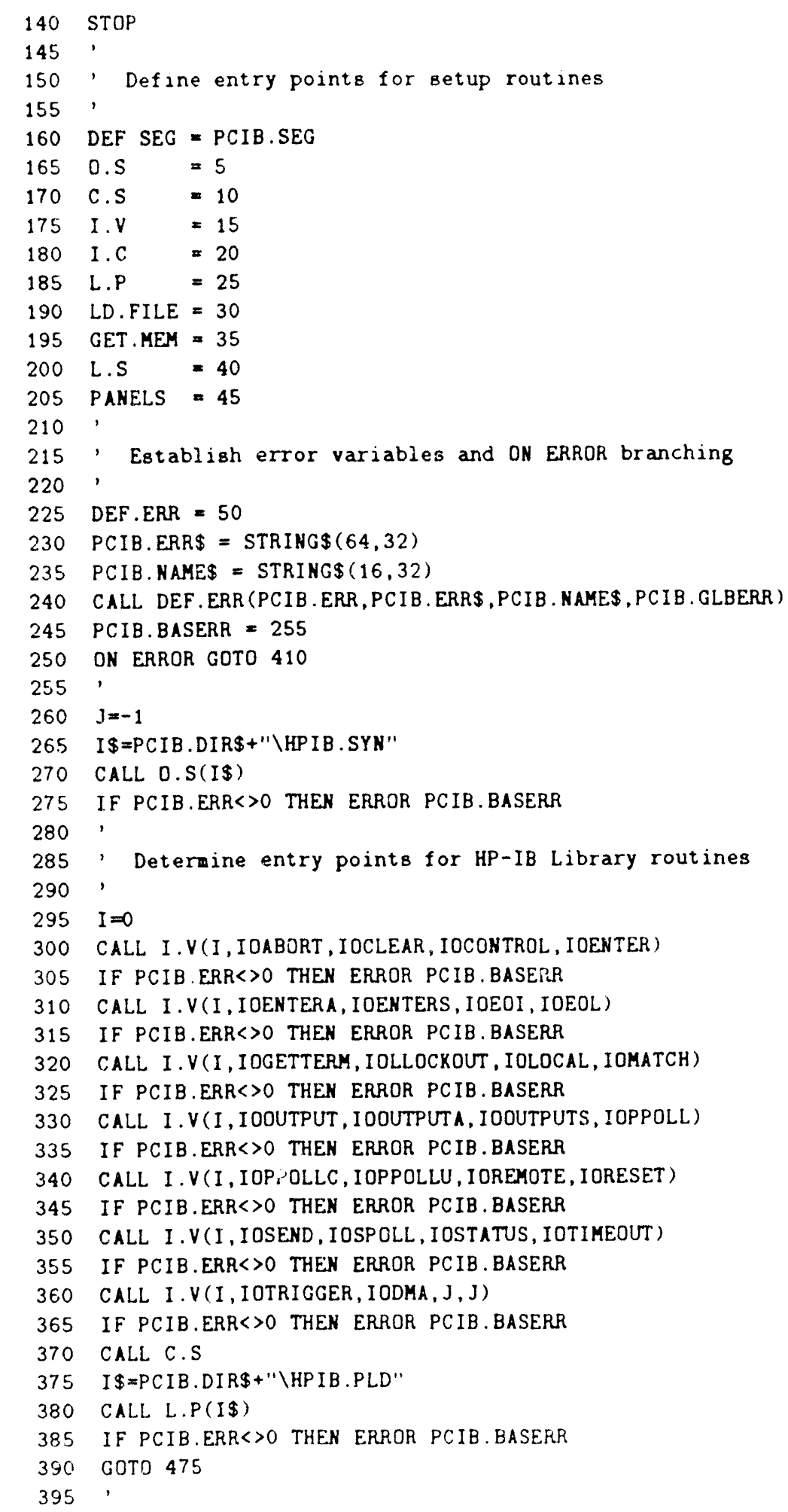




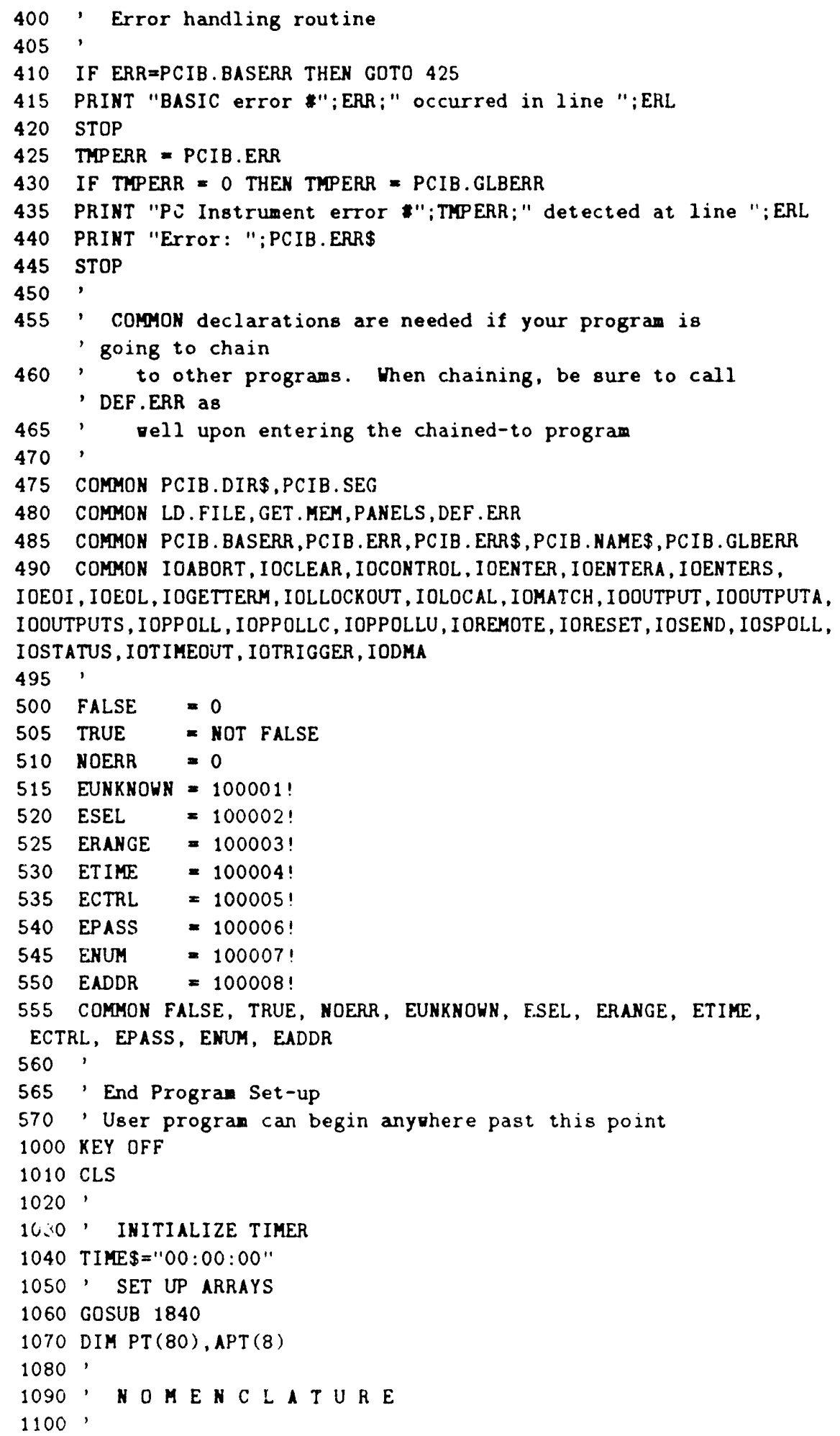




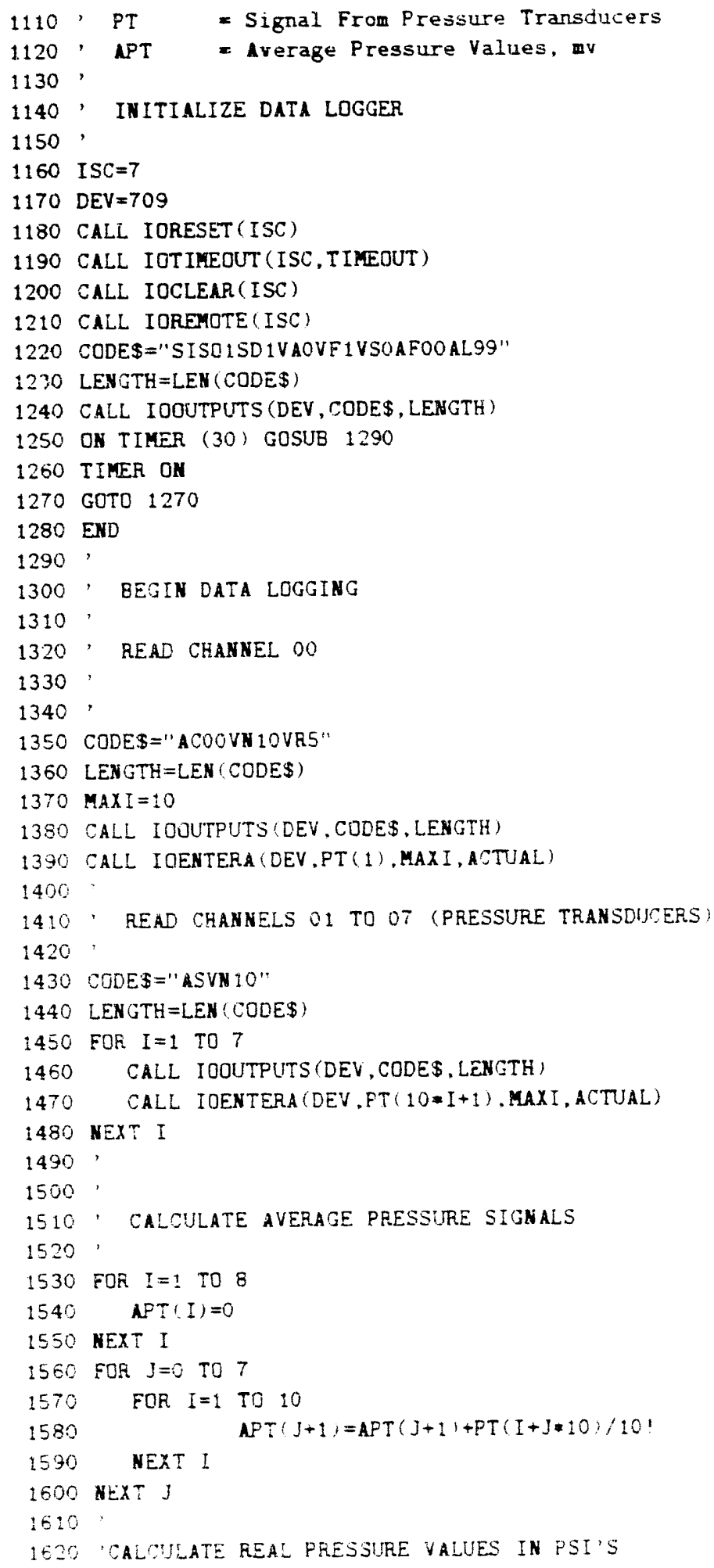




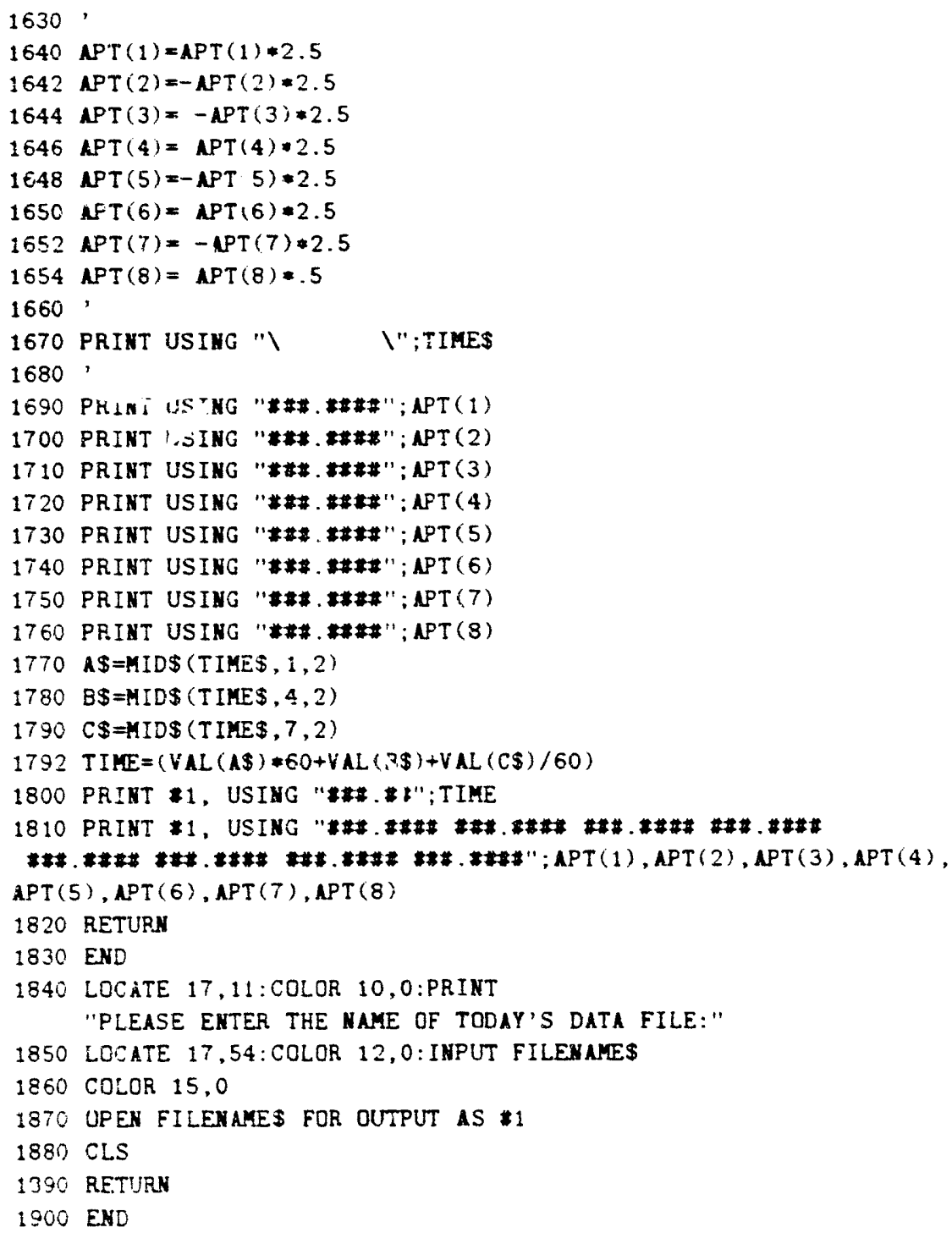


Program for plotting and smoothing of pressure data

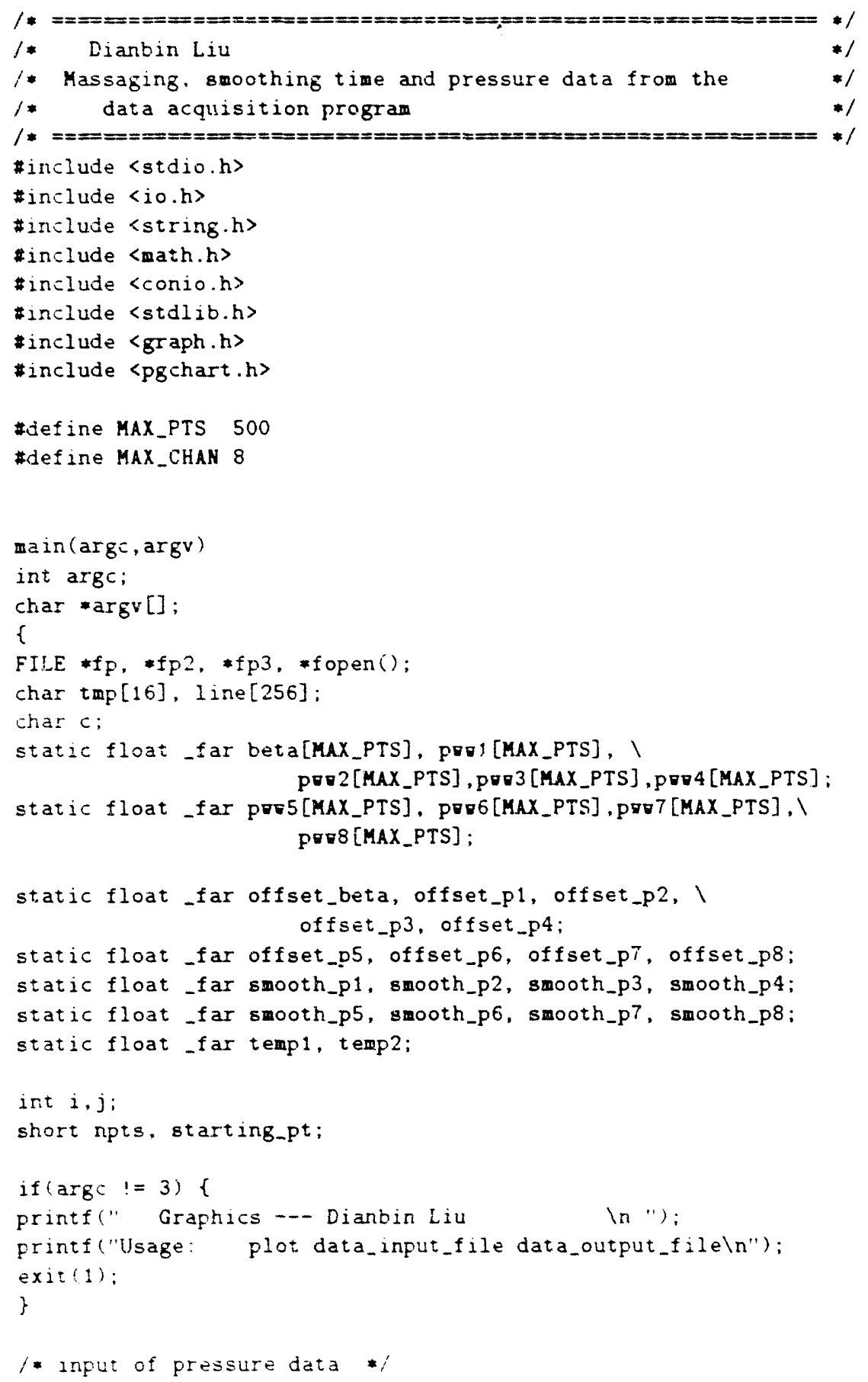




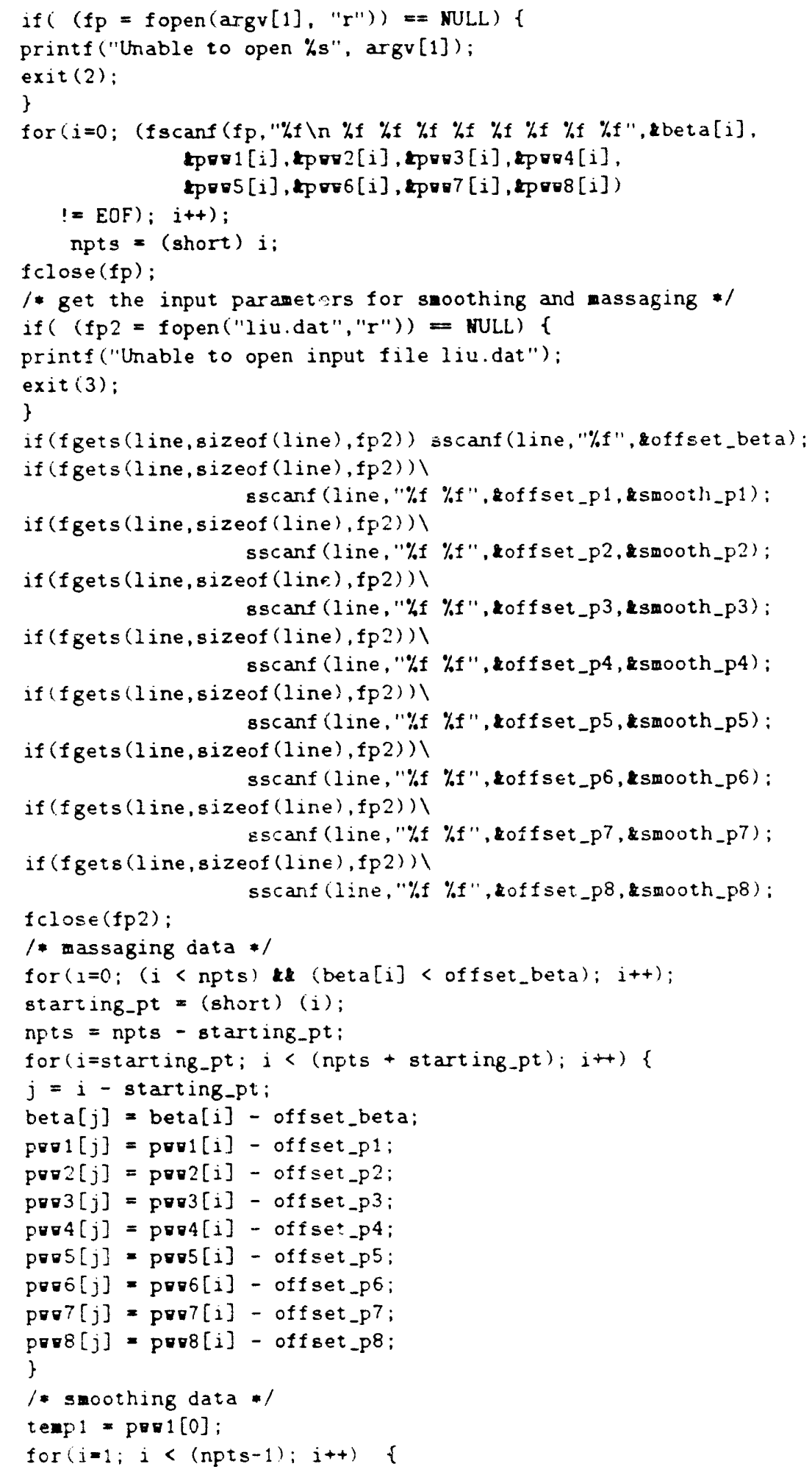




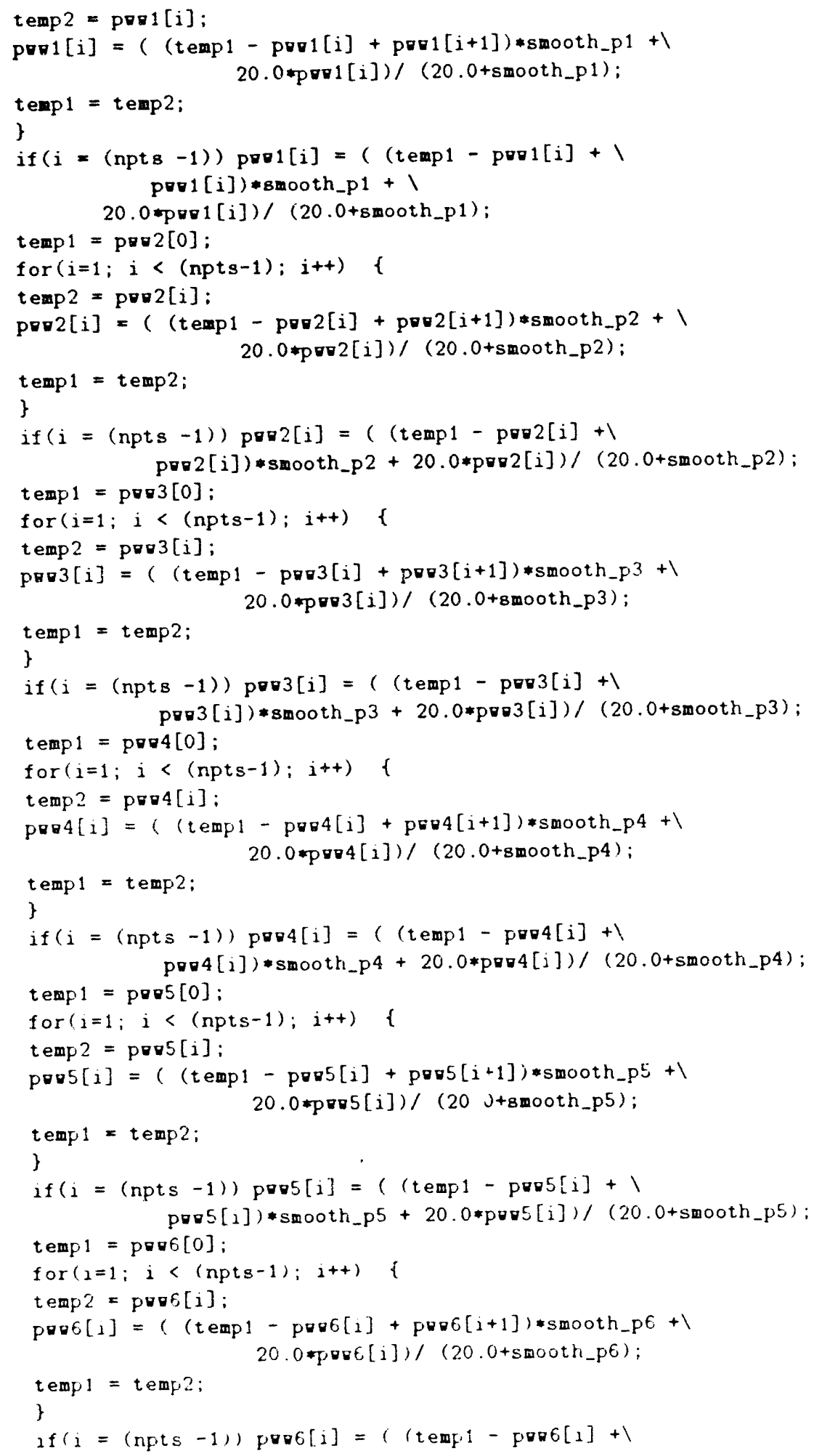




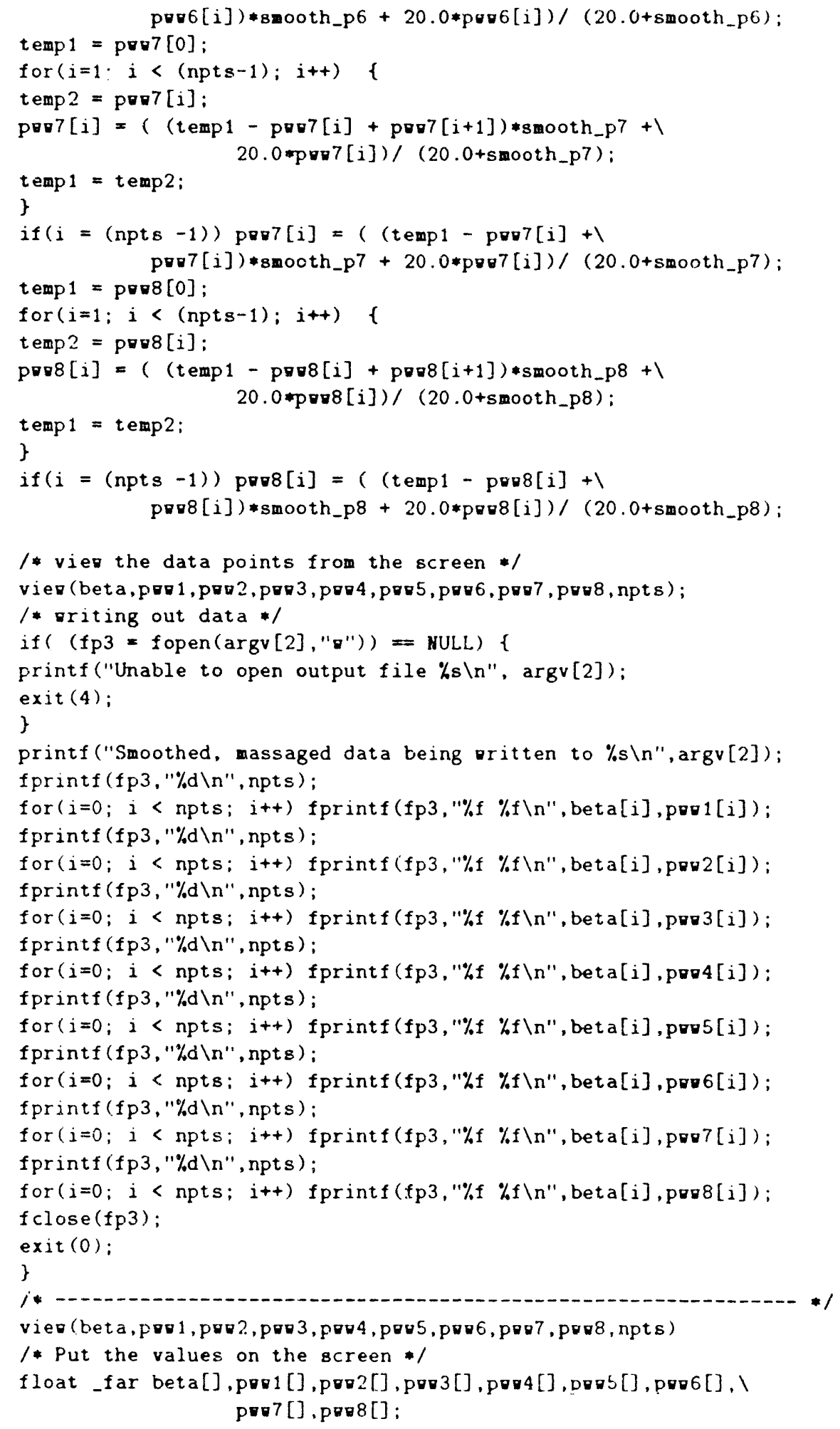




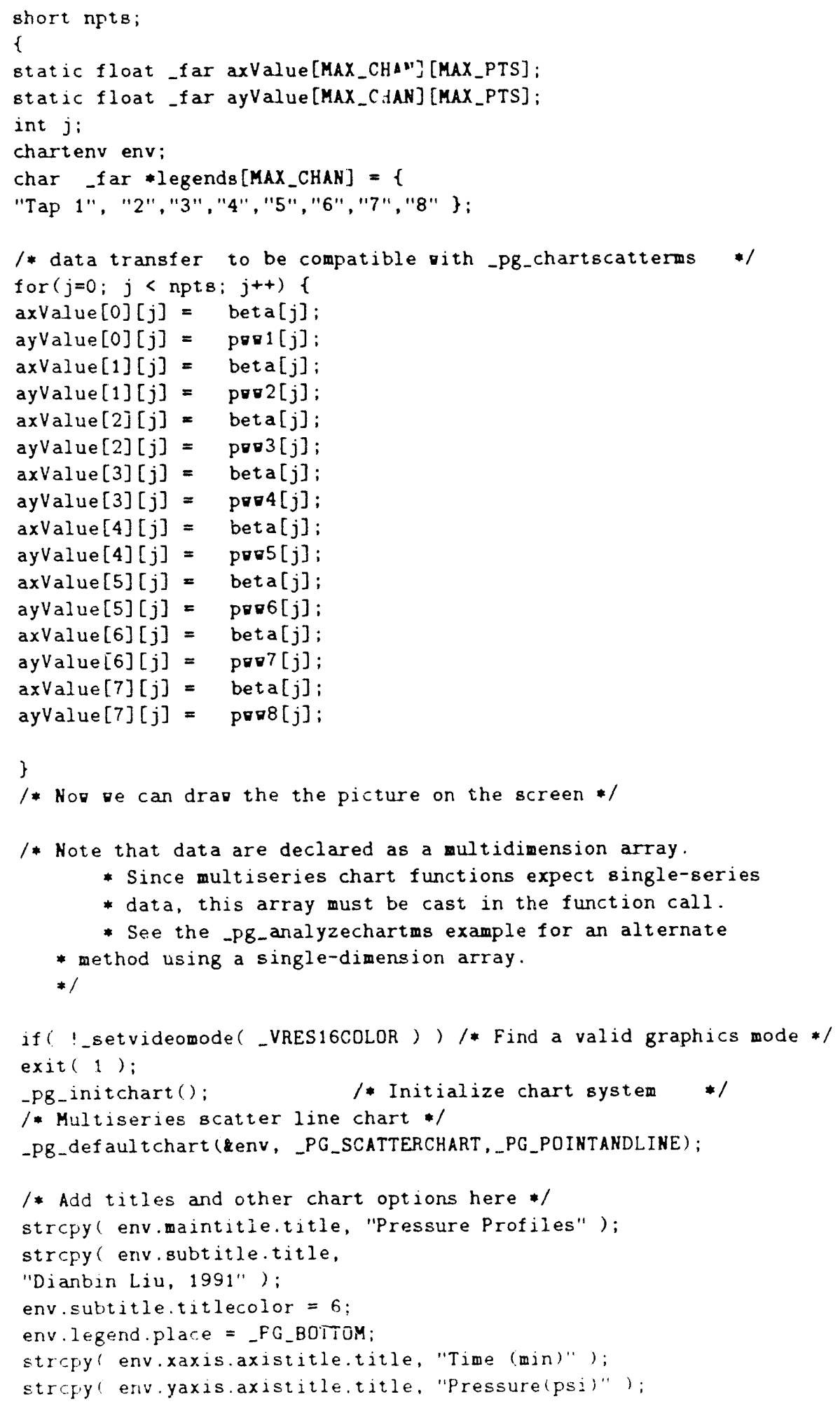


-Pg_chartscatterms( kenv, *axValue, *ayValue,

MAX_CHAN, npts, MaX_PTS, legends);

getch ():

-setvideomode ( DEFAULTMODE):

\} 


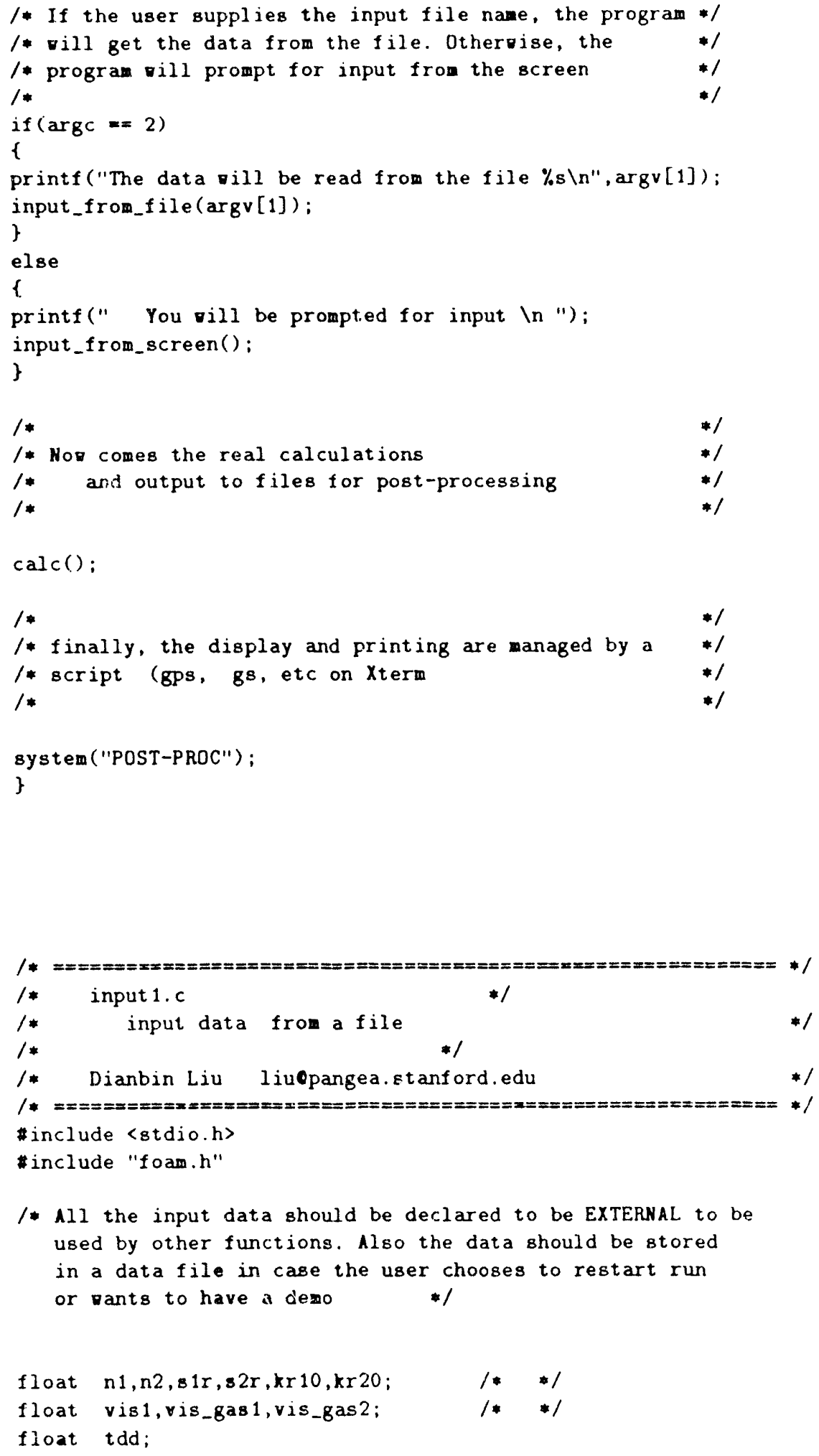




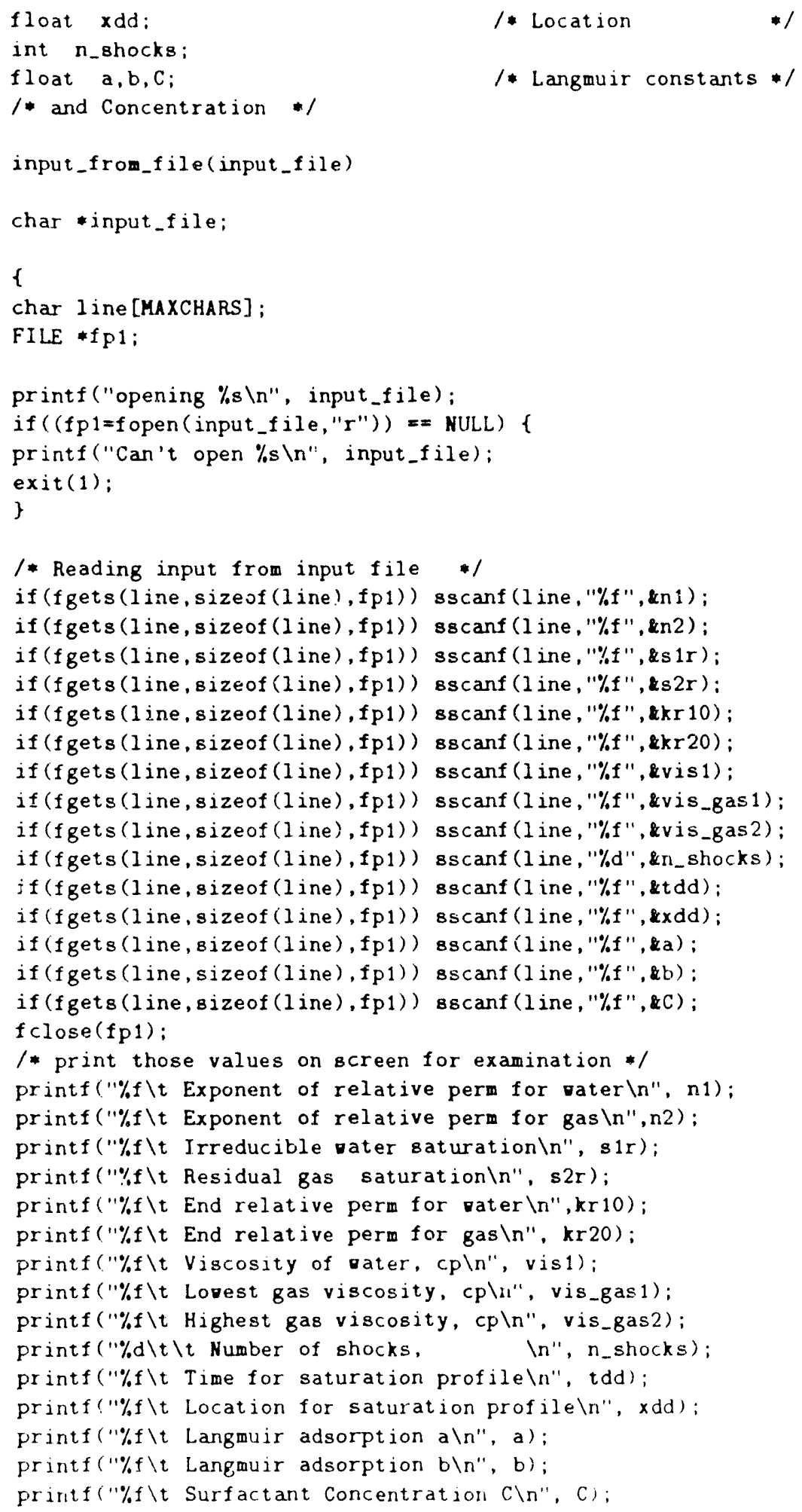


return;

\}

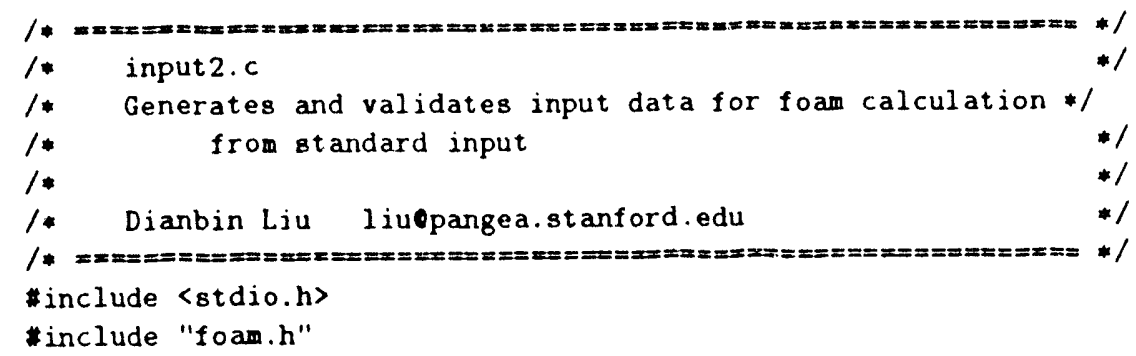

1* All the input data should be declared to be EXTERNAL to be used by other functions. Also the data should be stored in a data file in case the user chooses to restart run or vants to have a demo */

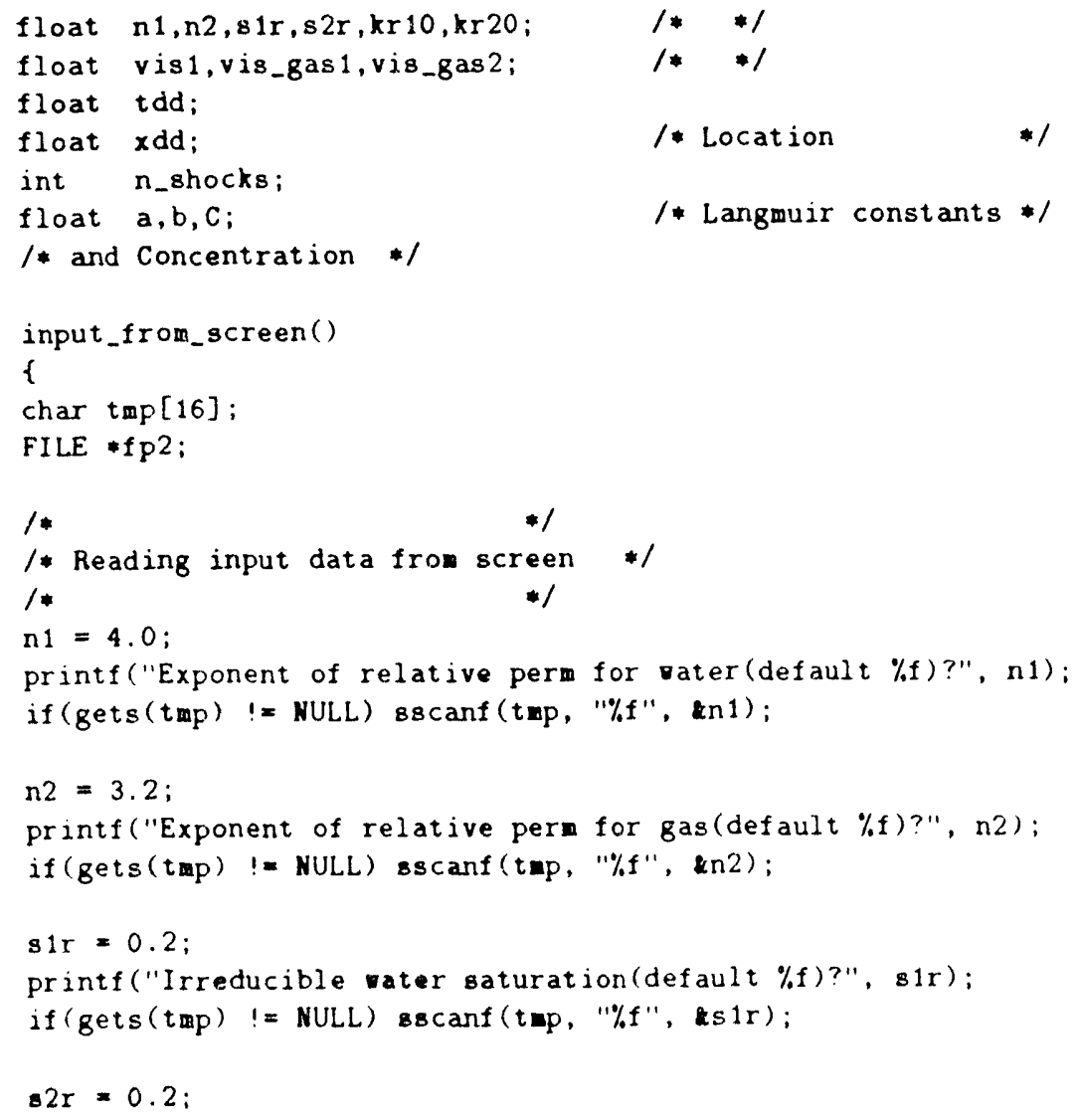




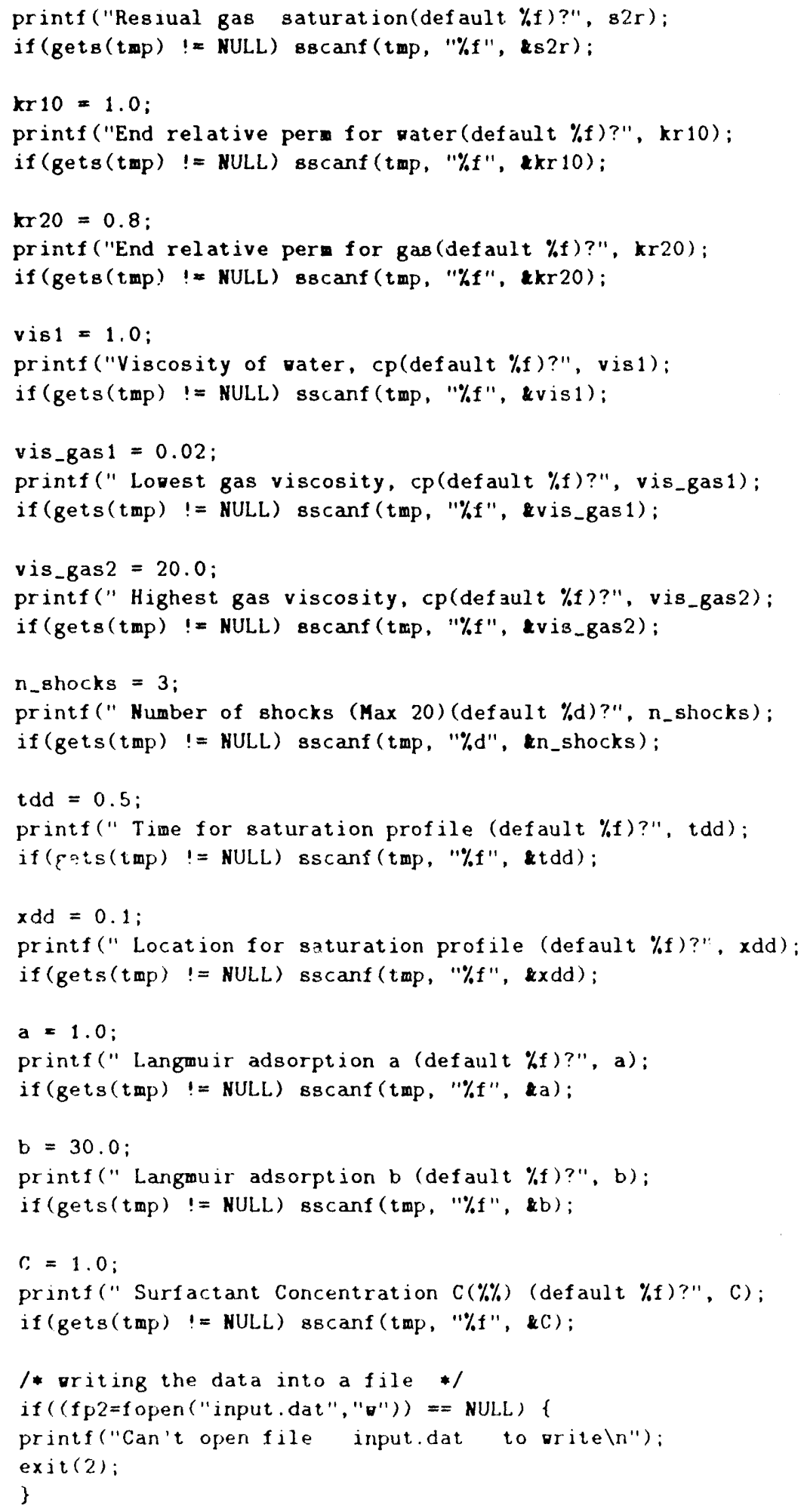




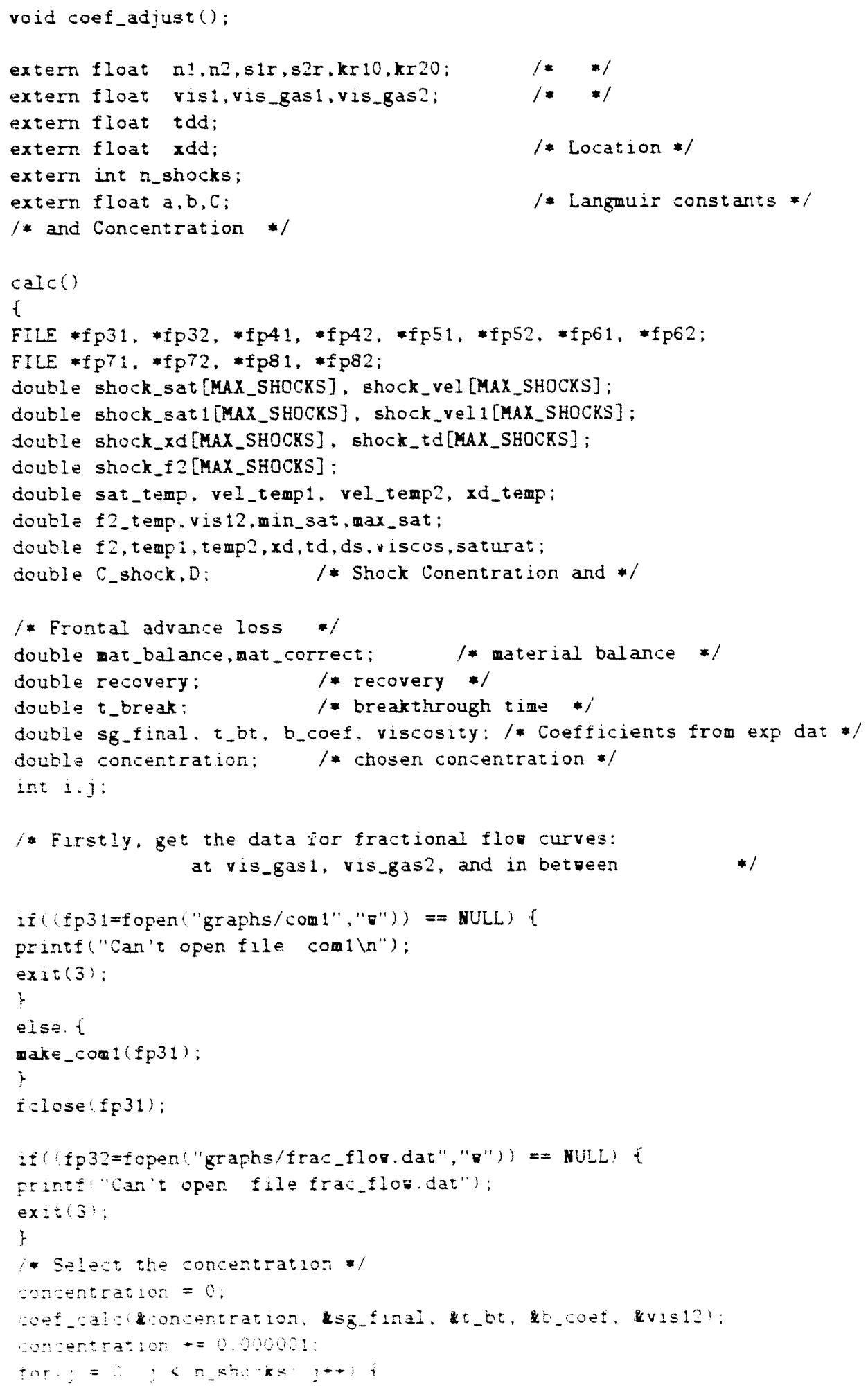




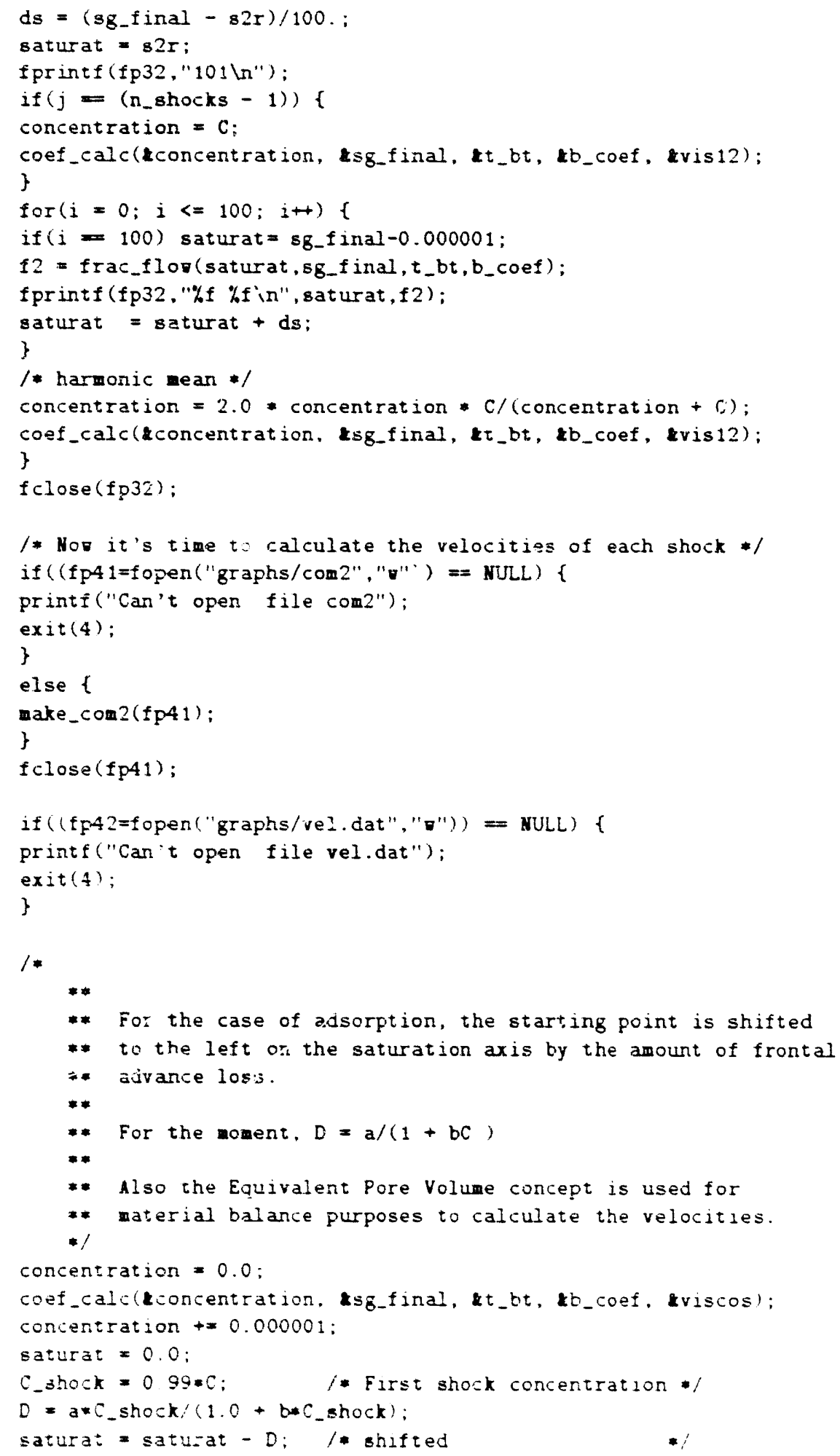




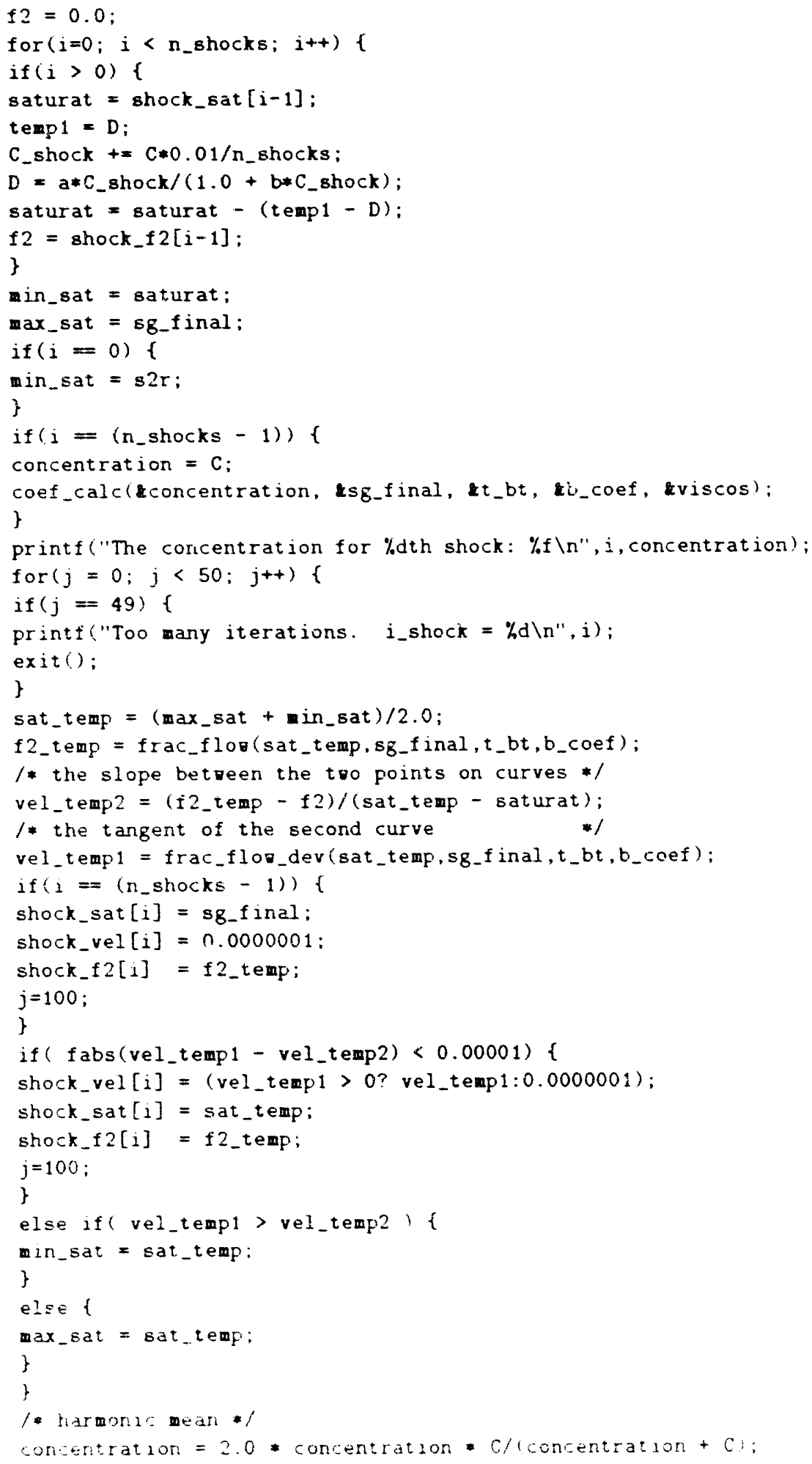




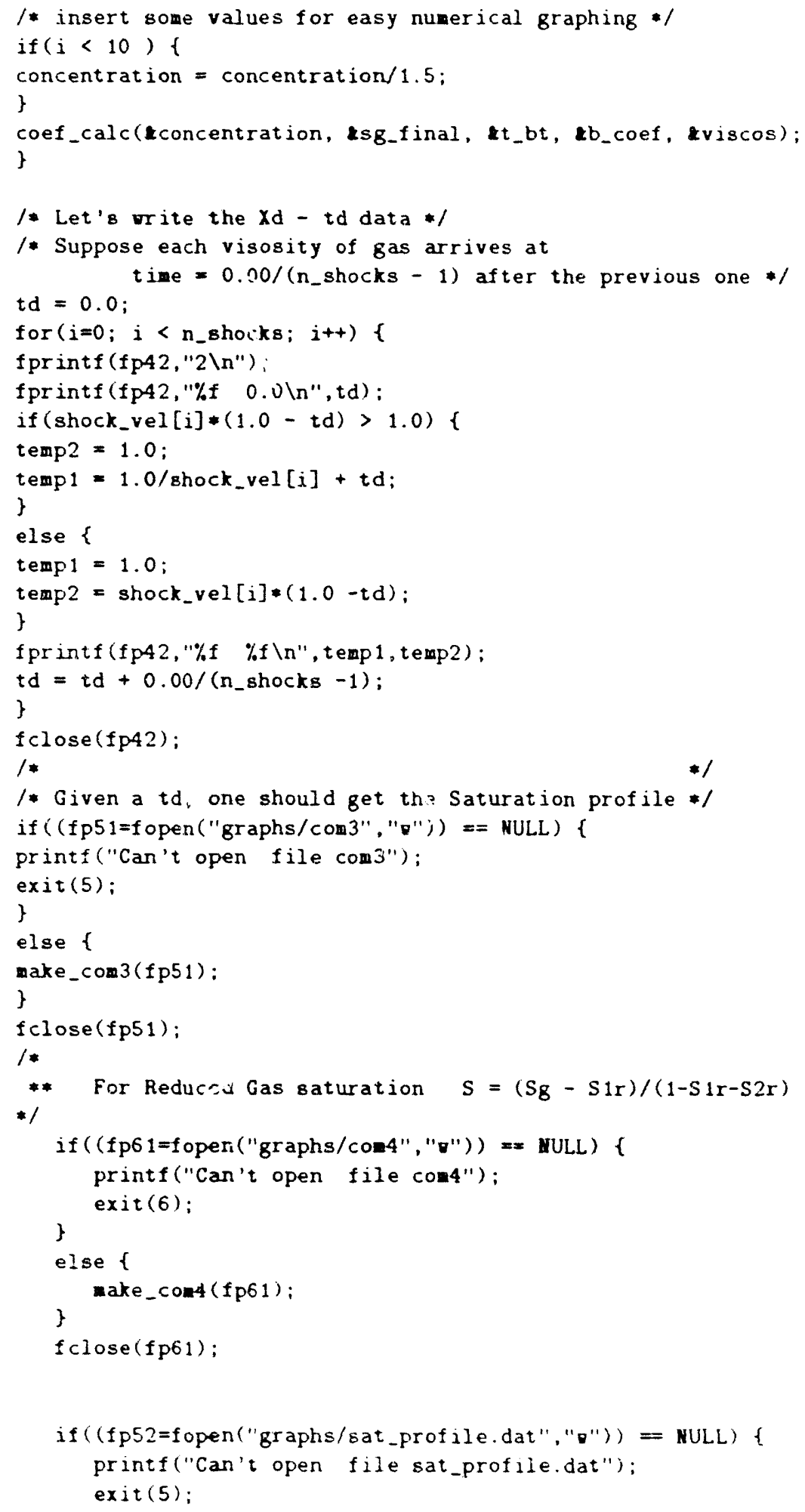




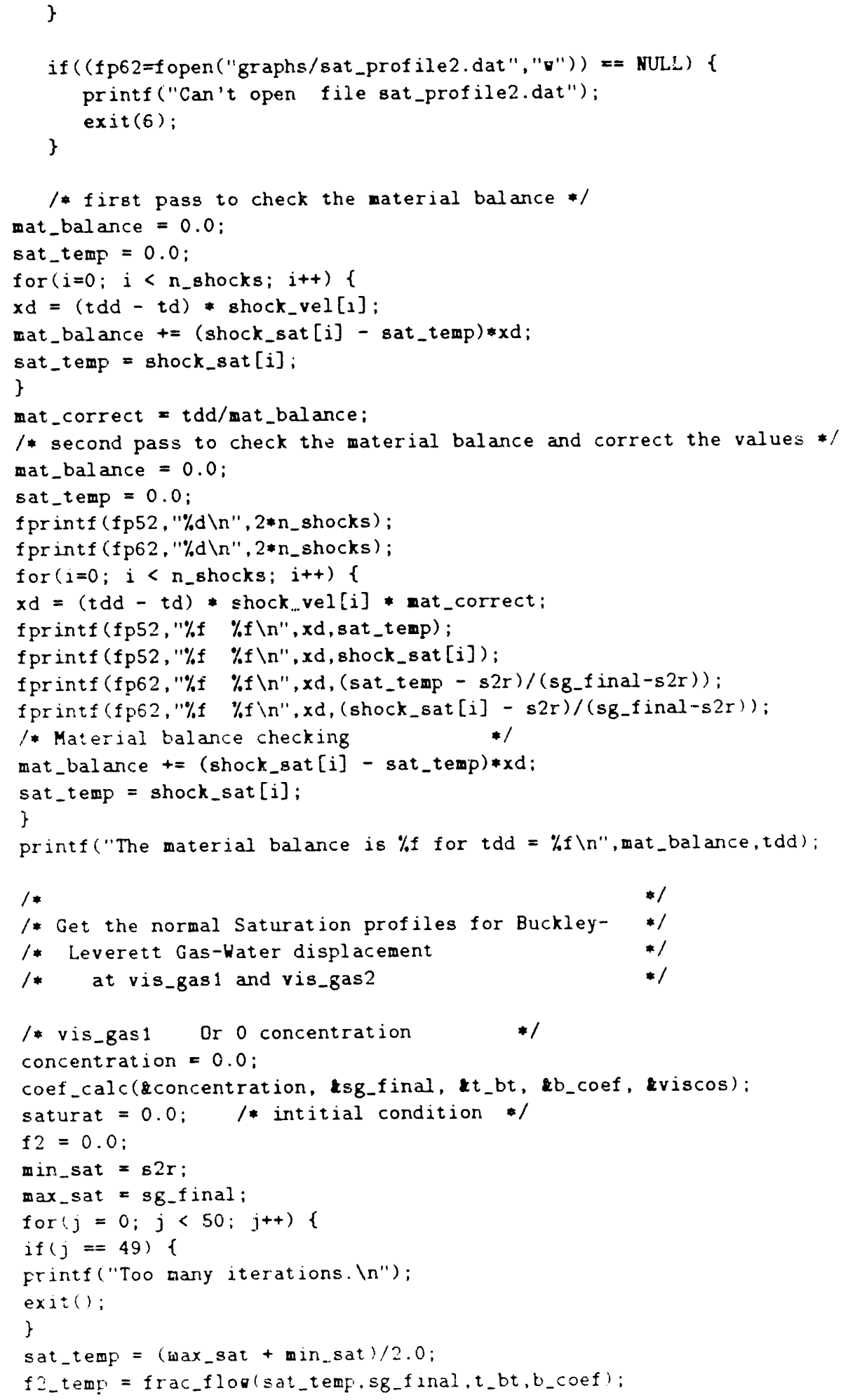




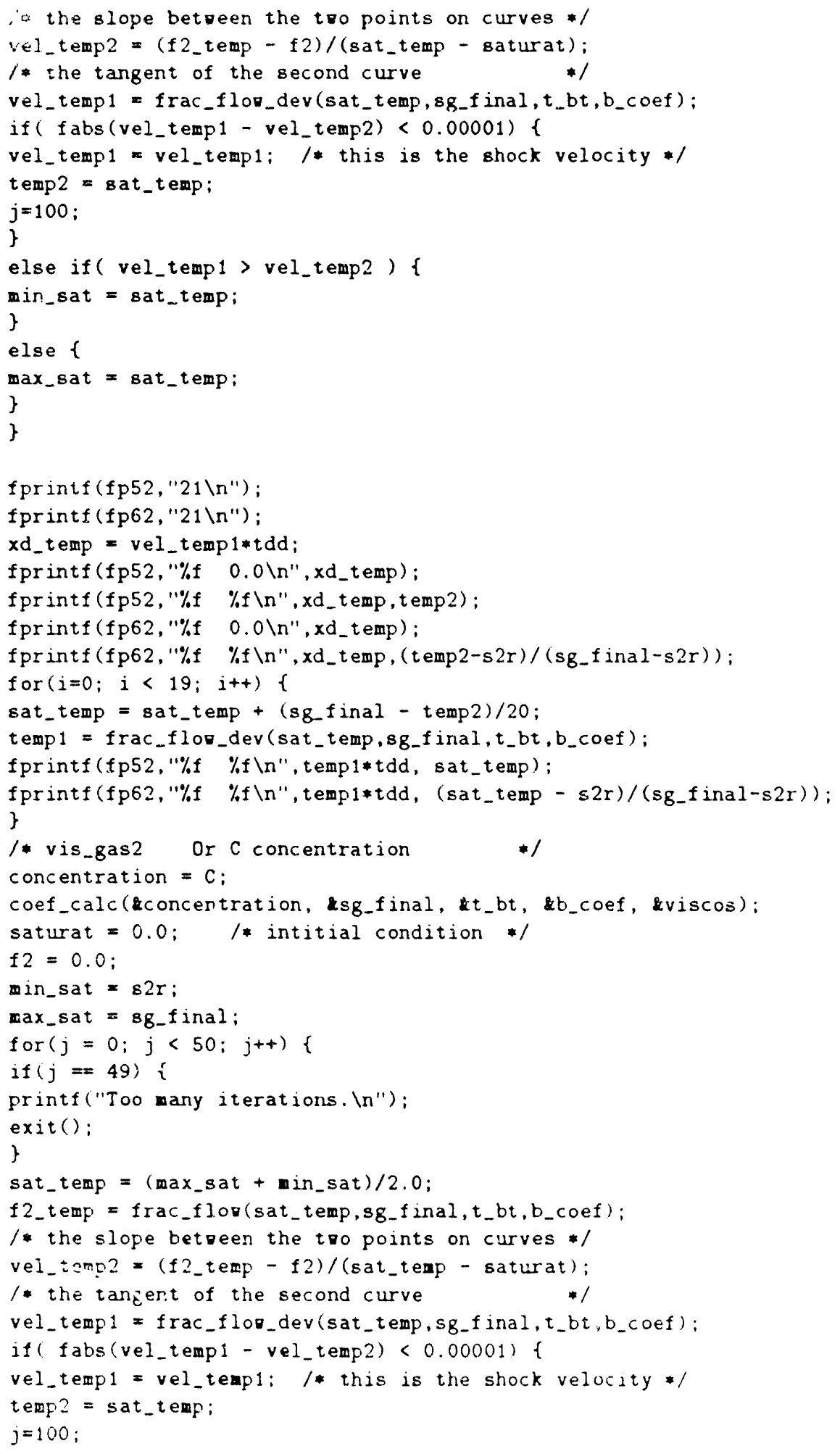




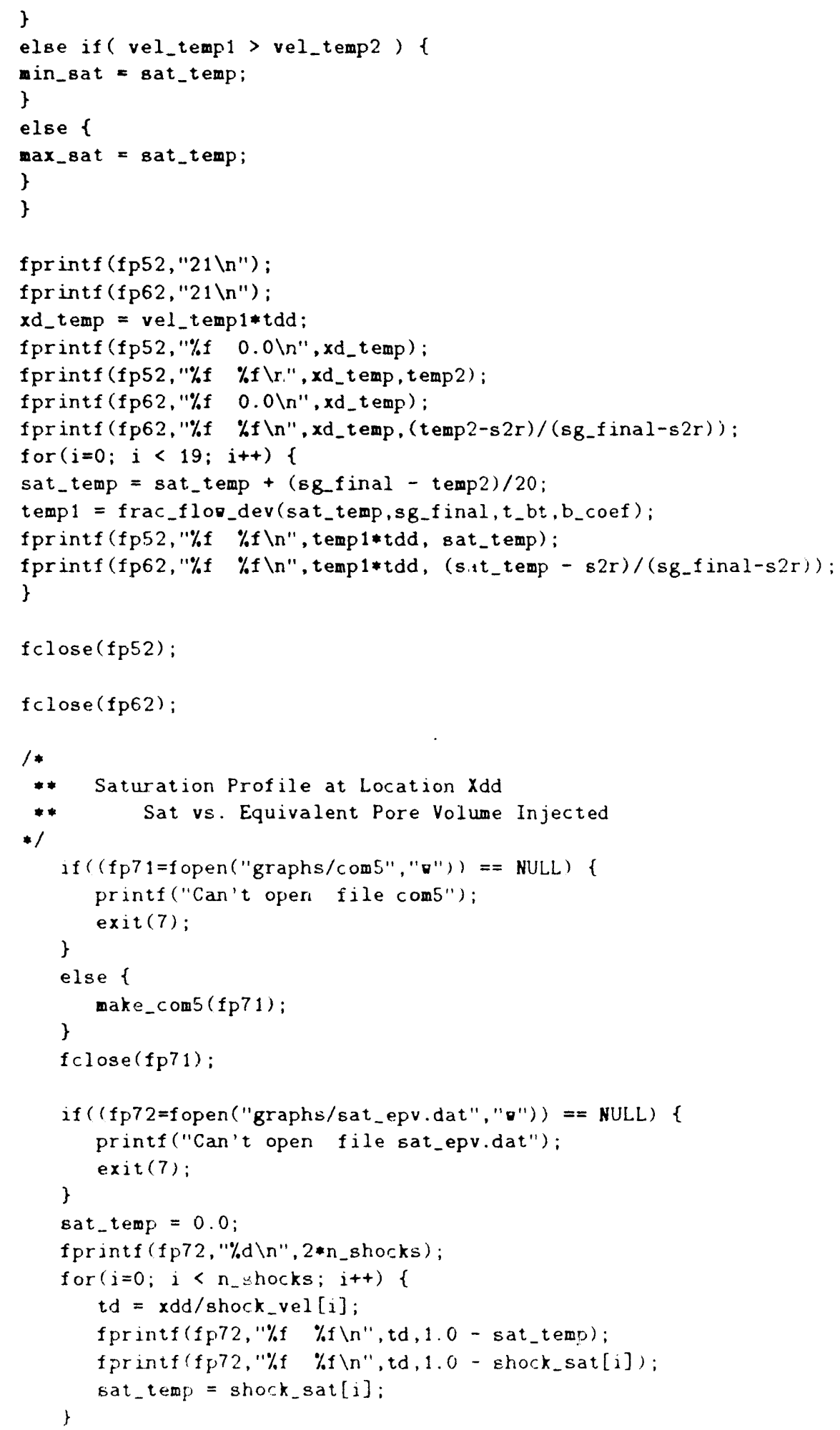




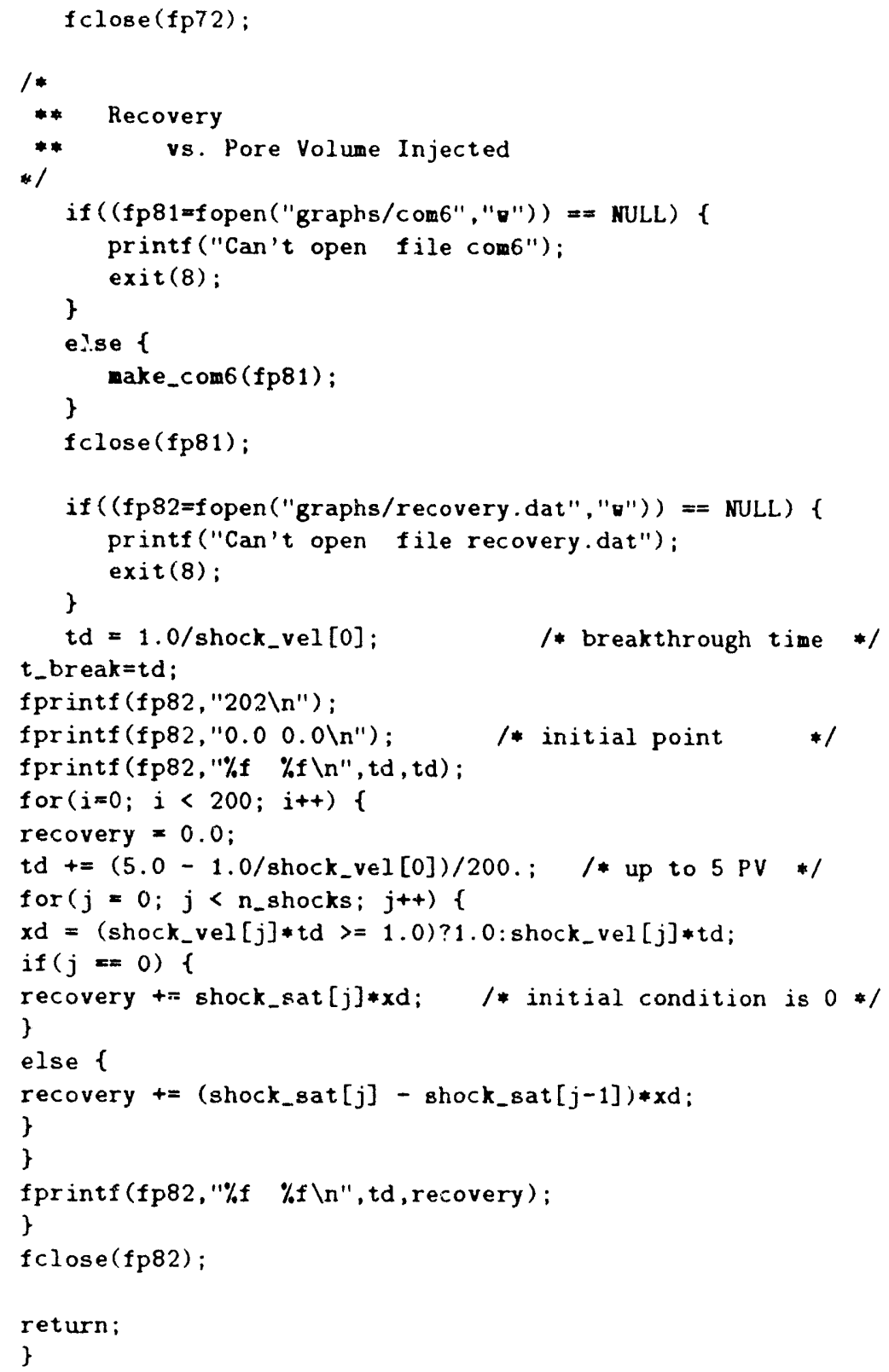




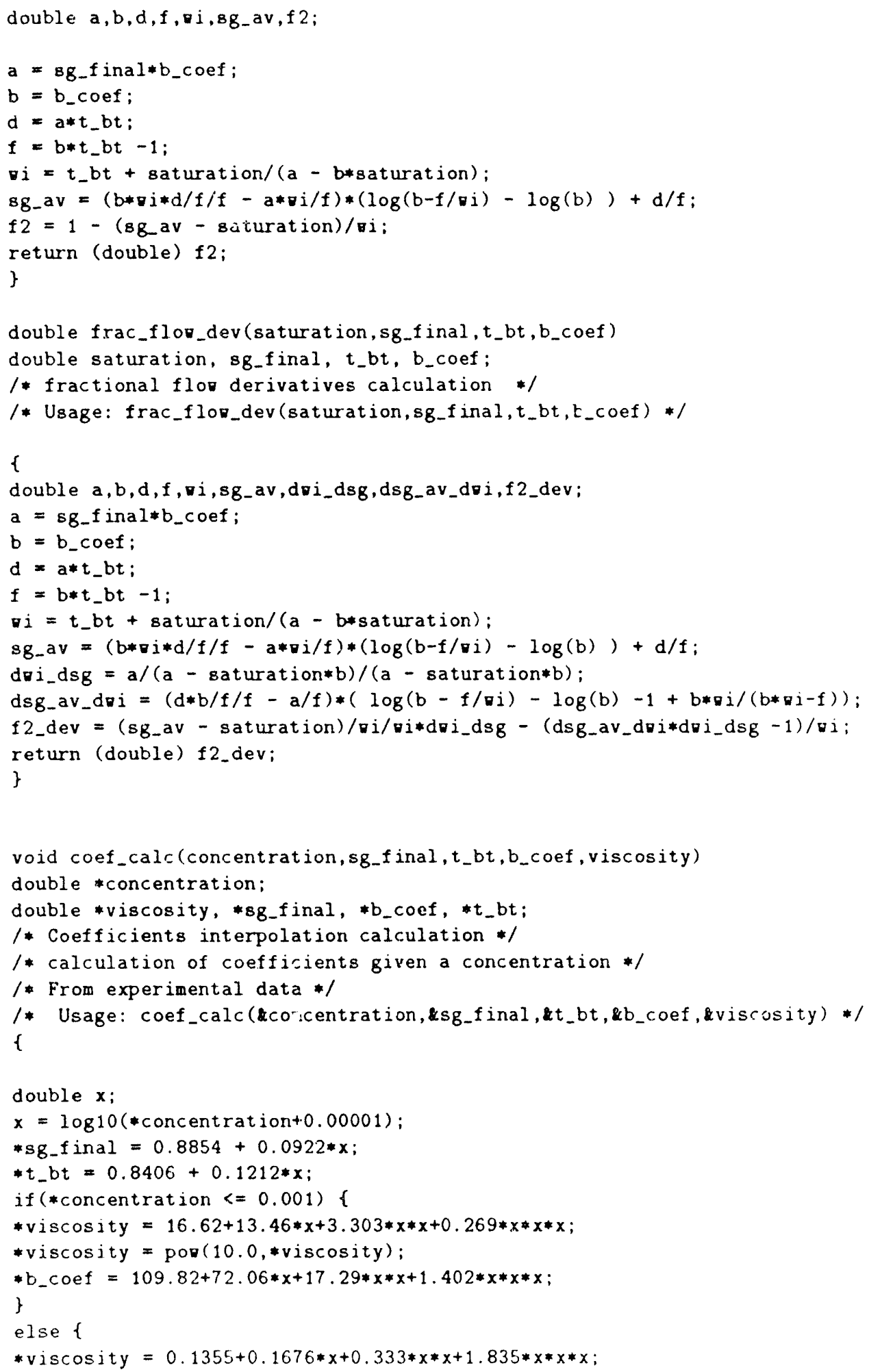




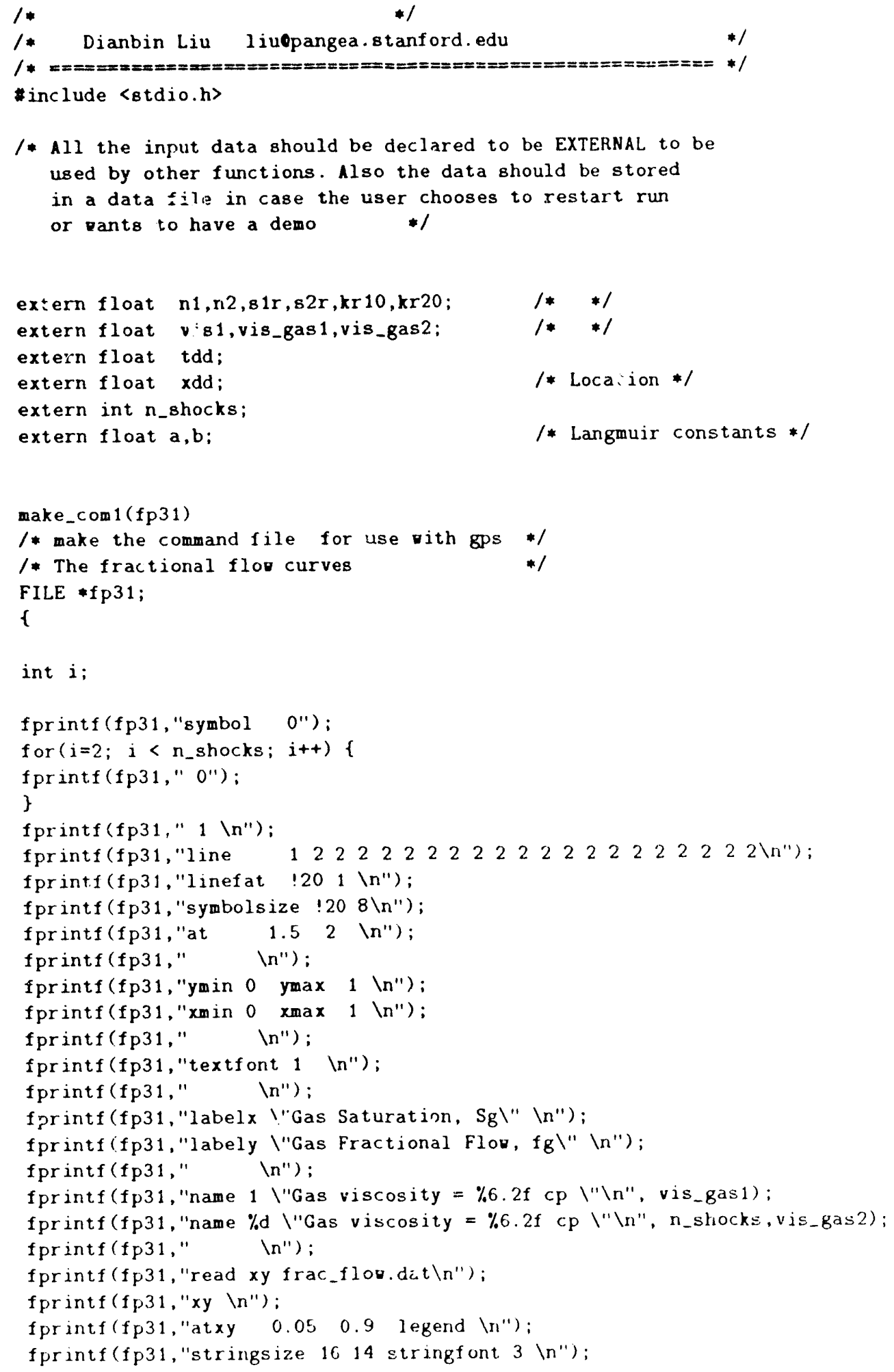




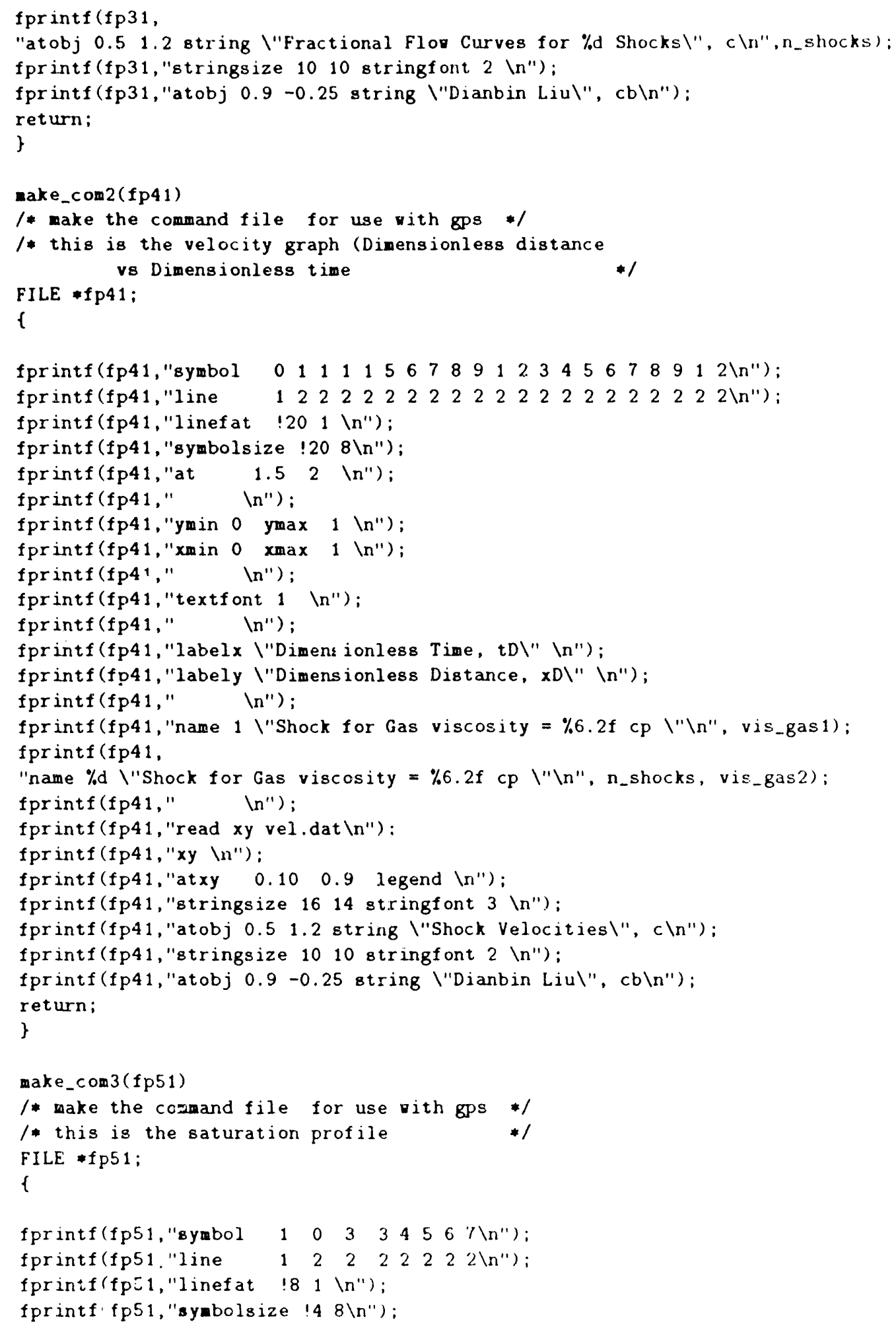




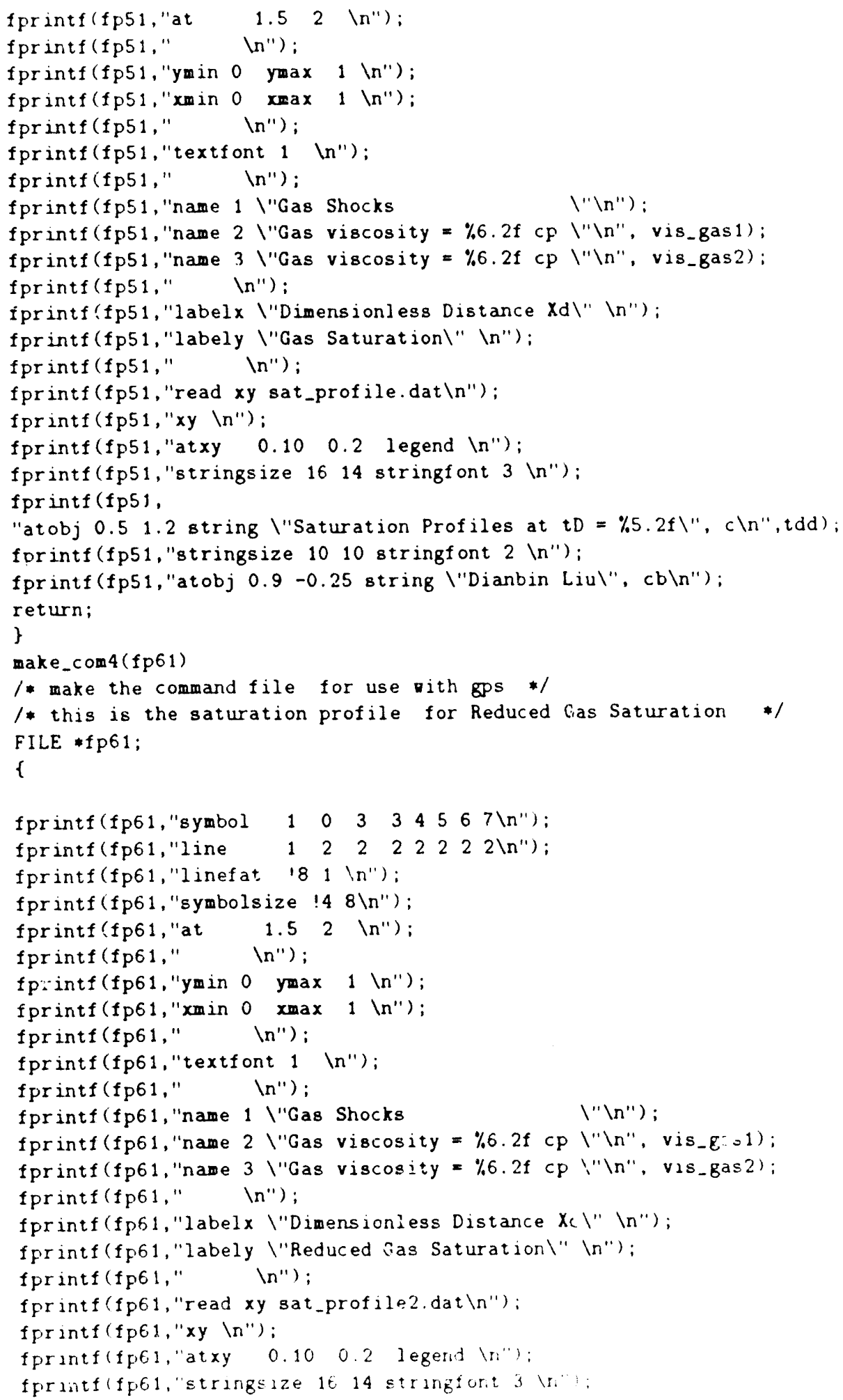




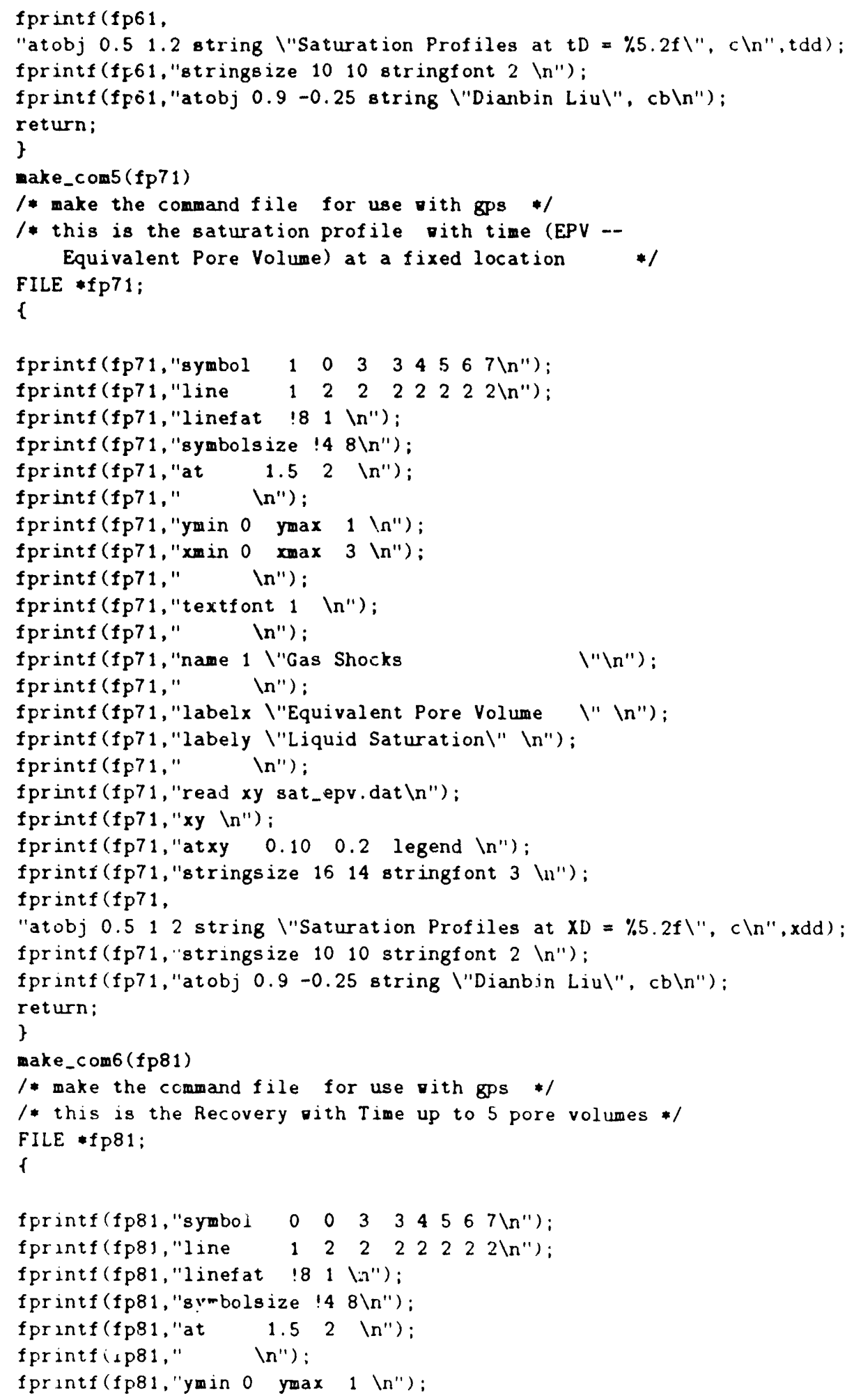




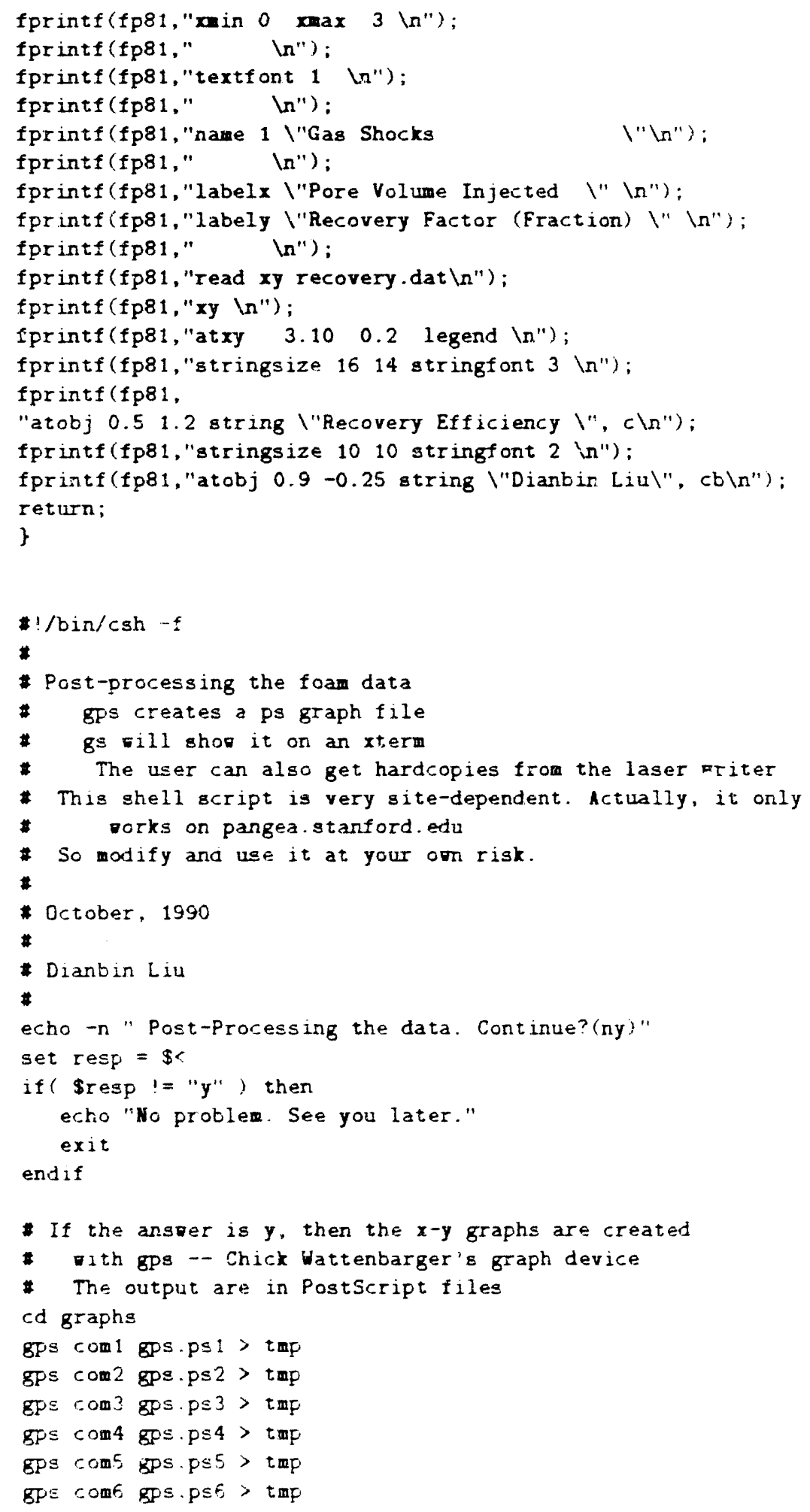




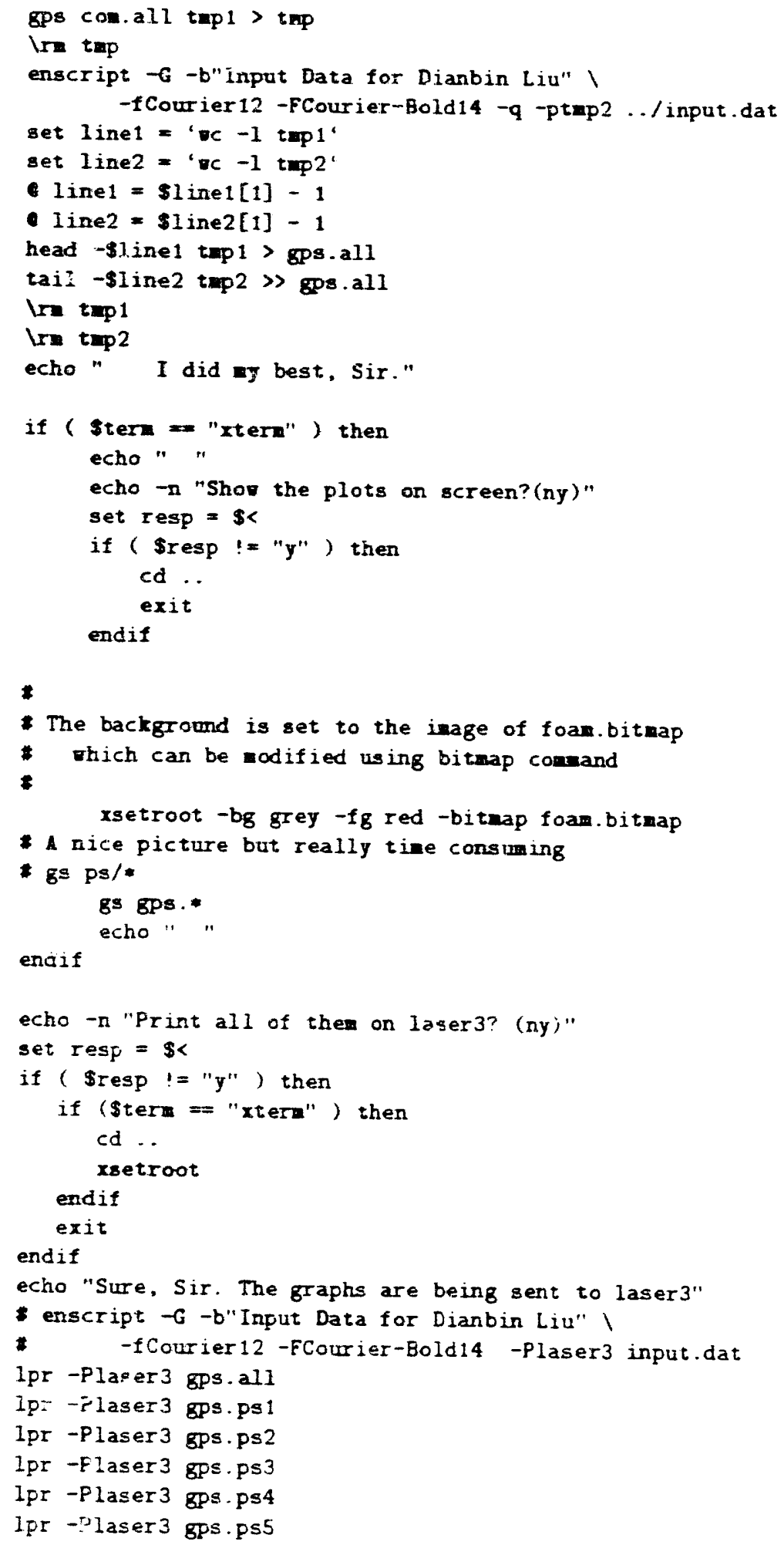




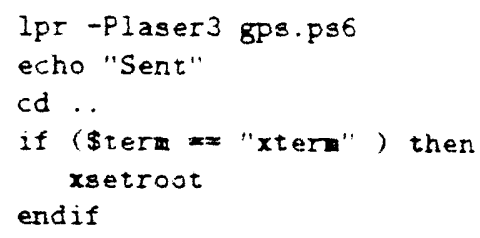




\section{Appendix D}

\section{Computer Program of Mixing Effect}

Program to find the mixing coefficients, $C$ and $\alpha$

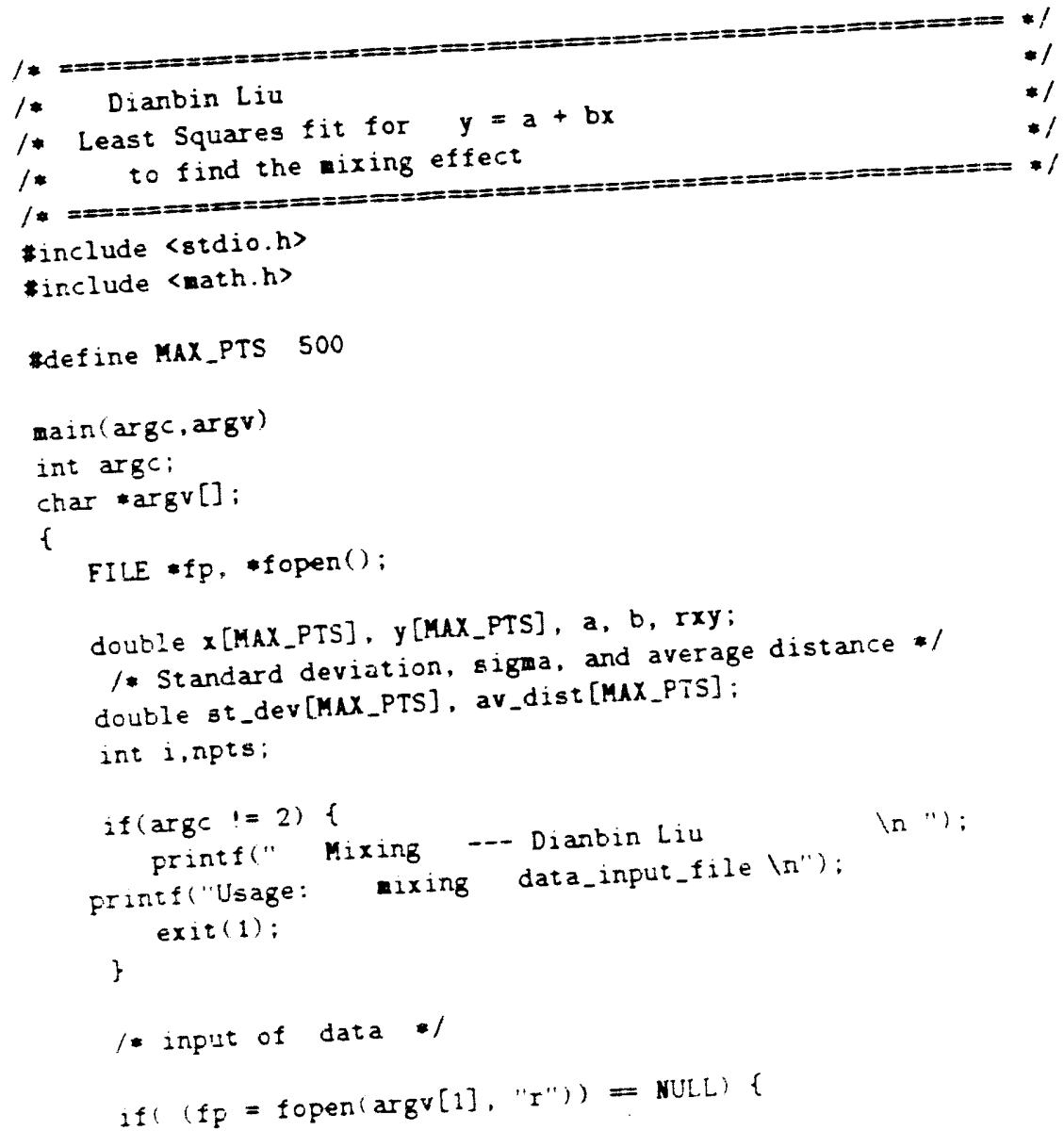




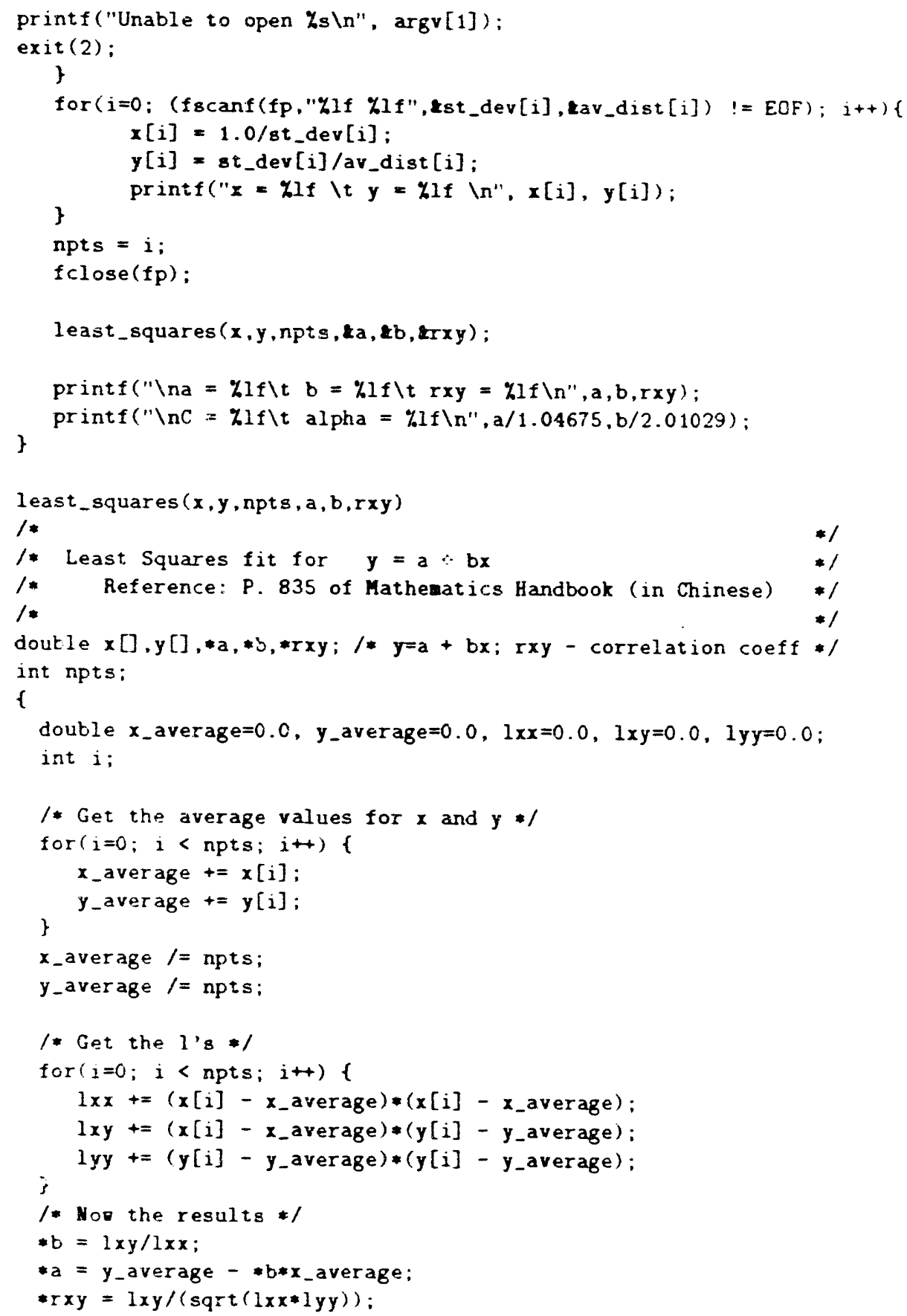




\section{Appendix E}

\section{Mixing Effect on Foam Displacements}

Taking Run 41 as an example, a detailed calculation is shown here to matcli the foam displacement saturation histories using the mixing coefficients.

Having found the mixing coefficients, $C$ and $\alpha$, in Chapter 6 , the standard deviation of each distance traveled by a corresponding saturation can be calculated from Equation 6.5 , which is:

$$
\sigma=\frac{1.04675 C x+\sqrt{(1.04675 C x)^{2}+4 \times 2.01029 \alpha x}}{2}
$$

As discussed before. for each location $x, \sigma$ can be calculated from the above equation. Then the ratio of the $C x$ value to $\sigma$ gives rise to the multiplier that should be used to account for the mixing effect for that location. However, this procedure applies best when the saturation profiles are readily available. In this work, the saturation histories at different loctions were measured and are believed to be reliable.

So, some manipulation was needed to find the multipliers to be applied for each saturation history at each location.

In fact. replacing the distance with the inverse of time would convert a saturation profile to saturation history. Substituting this concept and Equation E.1 into the multiplier $C x / \sigma$. we get: 


$$
\frac{C x}{\sigma}=\frac{C}{l_{p} \sigma}=\frac{2 C}{1.0465 .5 C+\sqrt{\left(1.04655 C j^{2}+4 \times 2.010290 . T_{F} L\right.}}
$$

where $I_{p}$ is time in equivalent pore volumes injected. $X$ is the distance to that specific location from the inlet in $\mathrm{ft}$, and $\mathrm{L}$ is the length of the sandpack in $\mathrm{ft}$. For the long sandpack in this work, the length was $4 \mathrm{ft}$.

After the saturation history was calculated from the displacement theory for the first location close to the inlet, as listed in columns 1 and 2 in Table E.1. the multipliers are then found for different locations along the sandpack from Equation E.2. Note that the multipilers also depend on the equivalent pore volume injected. The multipliers for locations 2. 3. and $4(1.225,2.542$, and 3.433 ft from the inlet $)$ are calculated in Columns 3.4 . and 5 of Table E.1.

Finally, the EPV's for other locations are obtained by the product of the EPV of Location 1 and the multipliers of other locations. Then the EPV"s are plotted against the saturation as the final match of the saturation historis, shown in Figure 6.17.

By exactly the same procedures, the mixing multipliers were found and tabulated in Table E.2. The EPI's are plotted against the saturation as shown in Figure 6.24. 


\begin{tabular}{|c|c|c|c|c|}
\hline $\begin{array}{c}\text { EPV for } \\
\text { Location } 1\end{array}$ & $\begin{array}{l}\text { Saturation } \\
\text { (fraction) }\end{array}$ & $\begin{array}{c}\frac{C}{V_{p \sigma}} \\
\text { Location } 2\end{array}$ & $\begin{array}{c}\frac{C}{l_{p} \sigma} \\
\text { Location } 3\end{array}$ & $\begin{array}{c}\frac{C}{\Gamma_{r} \sigma} \\
\text { Location } 4\end{array}$ \\
\hline 0.338050 & 1.000000 & 0.759143 & 0.653594 & 0.605507 \\
\hline 0.338050 & 0.995797 & 0.759143 & 0.653594 & $0.60550 \bar{i}$ \\
\hline 0.344783 & 0.991594 & 0.756583 & 0.650496 & 0.602304 \\
\hline 0.351516 & 0.983243 & 0.754054 & 0.647448 & 0.599159 \\
\hline 0.355919 & 0.974892 & 0.752417 & 0.645482 & 0.597133 \\
\hline 0.360 .323 & 0.967199 & 0.750792 & 0.643536 & 0.595130 \\
\hline 0.366689 & 0.959506 & 0.748466 & 0.640760 & 0.592275 \\
\hline 0.3730 .55 & 0.948790 & 0.746166 & 0.638025 & 0.589466 \\
\hline 0.382180 & 0.938075 & 0.742914 & 0.634174 & 0.585519 \\
\hline 0.391304 & $0.92383 i$ & 0.739713 & 0.630405 & 0.581662 \\
\hline 0.404353 & 0.909599 & 0.735221 & $0.6251 \overline{4}$ & 0.576295 \\
\hline 0.417402 & 0.891589 & $\overline{0.730826}$ & 0.620039 & 0.571094 \\
\hline 0.436165 & 0.873578 & 0.724669 & 0.612941 & 0.563889 \\
\hline 0.454929 & 0.851976 & 0.718696 & 0.606118 & 0.556985 \\
\hline 0.482300 & 0.830374 & 0.710291 & 0.596620 & 0.547411 \\
\hline 0.509671 & 0.805929 & 0.702230 & 0.587617 & 0.538375 \\
\hline 0.550639 & 0.781485 & 0.690751 & 0.574972 & 0.525746 \\
\hline 0.591606 & 0.755503 & 0.679915 & 0.563215 & 0.514065 \\
\hline 0.655186 & 0.729 .522 & 0.664157 & 0.546412 & 0.497472 \\
\hline $0.71936 i$ & 0.703644 & 0.649618 & 0.531200 & 0.482547 \\
\hline 0.825270 & 0.677766 & 0.627787 & 0.508847 & 0.460778 \\
\hline 0.931173 & 0.653618 & 0.608320 & 0.489314 & 0.441961 \\
\hline 1.278160 & 0.629471 & 0.556 .584 & 0.439489 & 0.394339 \\
\hline 1.625140 & 0.595082 & 0.517329 & 0.4032 .25 & 0.360200 \\
\hline 2.816310 & 0.560692 & 0.430268 & 0.326814 & 0.289432 \\
\hline $4.00 T 480$ & 0.504078 & 0.378169 & $0.28329 i$ & 0.249757 \\
\hline
\end{tabular}

Table E.1: Calculation of Mixing Multipliers for Run 41 


\begin{tabular}{|c|c|c|c|c|}
\hline $\begin{array}{c}\text { EPV for } \\
\text { Location } 1 \\
\end{array}$ & $\begin{array}{c}\text { Saturation } \\
\text { (fraction) }\end{array}$ & $\begin{array}{c}\frac{C}{V_{p \sigma}} \\
\text { Location } 2 \\
\end{array}$ & $\begin{array}{c}\frac{C}{V_{p \sigma}} \\
\text { Location } 3 \\
\end{array}$ & $\begin{array}{c}\frac{C}{\Gamma_{p} \sigma} \\
\text { Location } 4 \\
\end{array}$ \\
\hline 0.337168 & 1.000000 & 0.759481 & 0.654004 & 0.605931 \\
\hline 0.337168 & 0.996856 & 0.759481 & 0.654004 & 0.605931 \\
\hline 0.343414 & 0.993712 & 0.757101 & 0.651121 & 0.602951 \\
\hline 0.349660 & 0.986449 & 0.754748 & 0.648283 & 0.600021 \\
\hline 0.353720 & 0.979185 & 0.753233 & 0.646461 & 0.598142 \\
\hline 0.357779 & 0.972112 & 0.751729 & 0.644658 & 0.596281 \\
\hline 0.363706 & 0.965039 & 0.749553 & 0.642055 & 0.593607 \\
\hline 0.369632 & 0.954950 & 0.747400 & 0.639490 & 0.590971 \\
\hline 0.378179 & 0.944860 & 0.744334 & 0.6358 .53 & 0.587239 \\
\hline 0.386726 & 0.931199 & 0.741313 & 0.632286 & 0.583587 \\
\hline 0.398992 & 0.917537 & 0.737054 & 0.627288 & 0.578479 \\
\hline 0.411258 & 0.899977 & 0.732883 & 0.622426 & $0.57352: 3$ \\
\hline 0.428913 & 0.882417 & 0.727026 & 0.615651 & 0.5666336 \\
\hline 0.446568 & 0.861063 & 0.721335 & 0.609126 & 0.560025 \\
\hline 0.472284 & 0.839708 & 0.713326 & 0.600036 & 0.550849 \\
\hline 0.497999 & 0.815252 & 0.705627 & 0.591398 & 0.542166 \\
\hline 0.536 .346 & 0.790797 & 0.691679 & 0.579271 & $0.5300: 3 i$ \\
\hline 0.574693 & 0.764528 & 0.684315 & 0.567969 & 0.518780 \\
\hline 0.634099 & 0.738258 & 0.669288 & 0.551846 & 0.502626 \\
\hline 0.693504 & $0.71185 i$ & 0.655368 & 0.537181 & $0.4 x+40 x$ \\
\hline 0.791012 & 0.685455 & 0.634567 & 0.515729 & 0.467460 \\
\hline 0.888520 & 0.660631 & 0.615905 & 0.496913 & 0.449230 \\
\hline 1.202540 & 0.635806 & 0.566580 & 0.448931 & 0.403291 \\
\hline 1.516560 & 0.600283 & 0.528596 & 0.413 .3506 & $0.369 \times 40$ \\
\hline 2.553160 & 0.564759 & 0.445357 & 0.339605 & 0.301269 \\
\hline 3.589770 & 0.537131 & 0.394036 & 0.296 .395 & 0.261655 \\
\hline 9.745560 & 0.484752 & 0.265180 & 0.1934131 & 0.169069 \\
\hline 15.90130 & 0.430059 & 0.215013 & 0.155155 & $0.1351 \%()$ \\
\hline
\end{tabular}

Table E.2: Calculation of Mixing Multipliers for Run 42 


\section{Appendix F}

\section{Pressure Calculation for Foam Flow}

To calculate the pressure drop across the sandpack for foam flow, the following procedures could be used.

Since the displacement theory would result in shocks, the pressure calculations should be treated accordingly over each shock segment, as illustrated in Figure F.1.

At a given time, e.g. 0.1 pore volume injected, the gas saturation could be calculated. Then each shock would be treated individually to get the pressure drops from standard Buckley-Leverett theory.

At a later time, e.g. 0.2 pore volume injected, the saturation profile has advanced to a longer distance. The saturation profile at later times should be calculated using the concept of mixing discussed before. Then the total pressure drop could be obtained by adding up the individual pressure drops associated with the shocks.

Before gas breakthrough, the pressure drop across the core or sandpack is the sum of the pressure drop behind the front and the pressure drop ahead of it, which is water in this work.

After gas breakthrough, the total pressure drop is the sum of all the individual pressure drops up to the end of the sand pack, as illustrated in Figure F.1 at 0.4 pore volumes injected. 


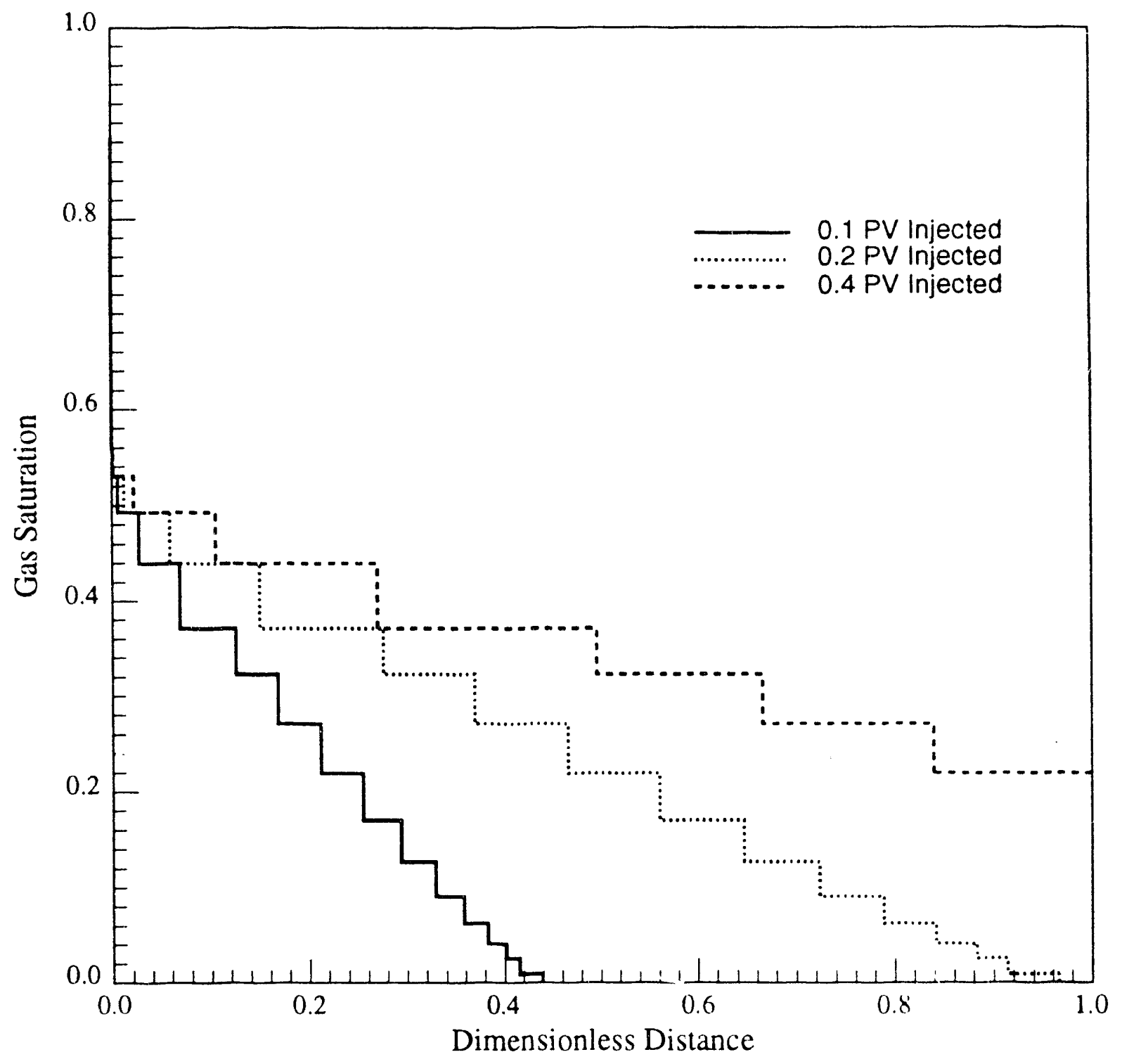

Figure F.1: Saturation Profiles for Pressure ('alculations 

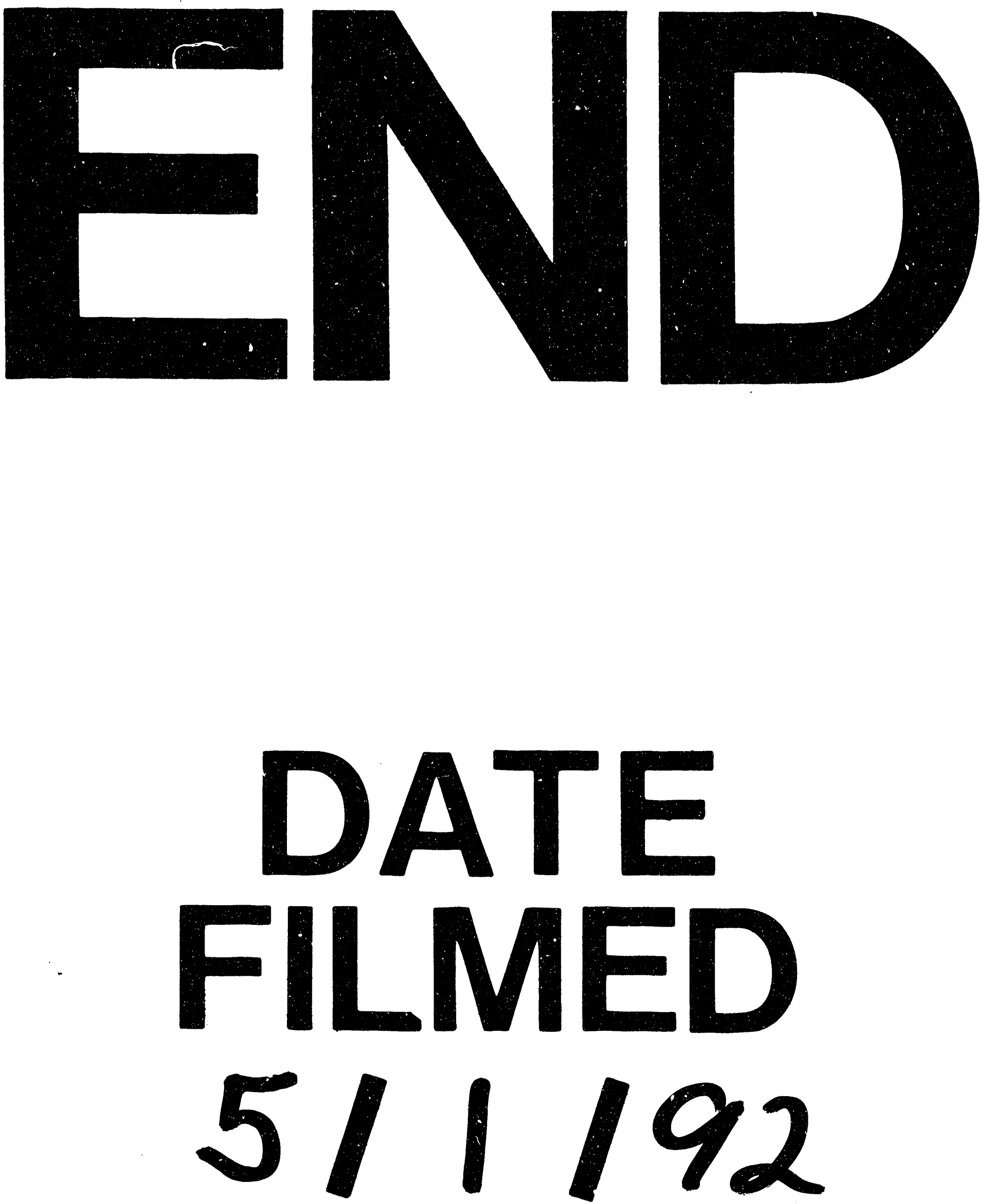